\title{
Development of a transparent indenter measurement system and indentation analysis for material mechanical property evaluation
}

\author{
Chuanyu Feng \\ West Virginia University
}

Follow this and additional works at: https://researchrepository.wvu.edu/etd

\section{Recommended Citation}

Feng, Chuanyu, "Development of a transparent indenter measurement system and indentation analysis for material mechanical property evaluation" (2005). Graduate Theses, Dissertations, and Problem Reports. 4147.

https://researchrepository.wvu.edu/etd/4147

This Dissertation is protected by copyright and/or related rights. It has been brought to you by the The Research Repository @ WVU with permission from the rights-holder(s). You are free to use this Dissertation in any way that is permitted by the copyright and related rights legislation that applies to your use. For other uses you must obtain permission from the rights-holder(s) directly, unless additional rights are indicated by a Creative Commons license in the record and/ or on the work itself. This Dissertation has been accepted for inclusion in WVU Graduate Theses, Dissertations, and Problem Reports collection by an authorized administrator of The Research Repository @ WVU.

For more information, please contact researchrepository@mail.wvu.edu. 


\title{
Development of a Transparent Indenter Measurement System and Indentation Analysis for Material Mechanical Property Evaluation
}

\author{
Chuanyu Feng \\ Dissertation submitted to the \\ College of Engineering and Mineral Resources \\ at West Virginia University \\ in partial fulfillment of the requirements \\ for the degree of \\ Doctor of Philosophy \\ in \\ Mechanical Engineering

$\begin{array}{ll}\text { Bruce S. Kang } & \text { Ph.D., Chair } \\ \text { Steven D. Woodruff } & \text { Ph.D. } \\ \text { Robert C. Creese } & \text { Ph.D. } \\ \text { James E. Smith } & \text { Ph.D. } \\ \text { Jacky C. Prucz } & \text { Ph.D. }\end{array}$

\section{Department of Mechanical and Aerospace Engineering West Virginia University \\ Morgantown, West Virginia \\ 2005}

Keywords: Spherical indentation, depth-sensing indentation, Transparent Indenter Measurement, stress-strain curve, in-situ indentation measurement, residual deformation, elastic recovery, interferometry, phase-shifting, fringe

Copyright (C) 2005 Chuanyu Feng 


\section{ABSTRACT}

\section{Development of a Transparent Indenter Measurement system and Indentation Analysis for Material Mechanical Property Evaluation}

\section{Chuanyu Feng}

Since Tabor showed the application of the spherical indentation approach to obtain material post-yielding true stress-strain curves, the indentation technique has been investigated to determine mechanical properties besides hardness measurement. With the development of various thin film materials, depth-sensing indentation is often applied to evaluate mechanical properties of thin film materials. However, indentation testing is a complicate mechanical process and analytical solutions are difficult to obtain. As a result, much of the understanding of the indentation process has been acquired through experiments and finite element simulations. Thus accurate measurement of indentation parameters is critical in the determination of surface mechanical properties using indentation method.

In this research, a Transparent Indenter Measurement (TIM) method was developed for material inspection and mechanical property measurement. The TIM method is capable of on-site, in-situ, (i) mechanical property measurement (hardness, Young's modulus, yield strength and post-yielding stress strain data) of material samples/machined parts and (ii) qualitative inspection of material surface conditions. The residual surface deformation after spherical indentations was first investigated on IN783 superalloy samples using phase-shifting moiré and Twyman-Green interferometry. The elastic-plastic boundary was identified based on the characteristic of the out-of-plane deformation fringe patterns. Then using the measured in-plane deformation, yield strength of the tested material was obtained. Using the TIM system, real-time in-situ measurement of indentation-induced out-of-plane deformation and contact radius are directly measured during an indentation process. Coupled with elastic recovery theories and 2D finite element analyses, a procedure was developed to determine the material stress-strain curve. It is also demonstrated that the TIM method is suitable for debonding inspection of thin film materials. 


\section{ACKNOWLEDGEMENTS}

I wish to give special thanks to my advisor, Dr. Bruce S. Kang, for his continuously support, encouragement and guidance during my study in West Virginia University. I c wish to extend my thanks to the committee members, Dr. Steven D. Woodruff, Dr. Robert C. Creese, Dr. James E. Smith, Dr. Jacky C. Prucz, for their time and efforts to review the proposal, this dissertation, and their comments on various topics.

I would like to thank all the members in Dr. Kang group, Julio Noriega, Dennis(Rodha Kamil), Srikanth Ramasubramanian, Lee, Venkatesh Parthasarathy, Ziheng Yao, et al, for their constructive suggestion and kindly help during this research work. The contributions are highly appreciated.

I must give special thanks to Diane Schwegler-Berry (NIOSH, Morgantown, WV) for her help on previous work with the Scanning Electron Microscope (SEM).

Finally, I must give my thanks to my family, especially to my wife, Dongxiang SUN, for her love, support and help during these years; my daughter, April Keshun Feng, who gave so many joys for the past several months. 


\section{TABLE OF CONTENTS}

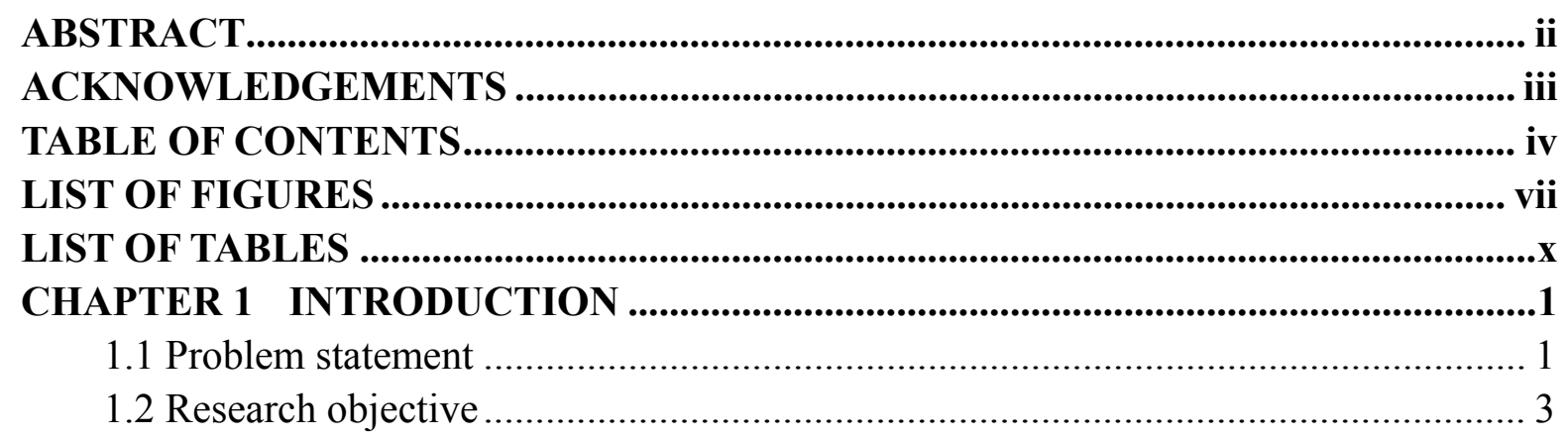

CHAPTER 2 LITERATURE REVIEW..................................................................................4

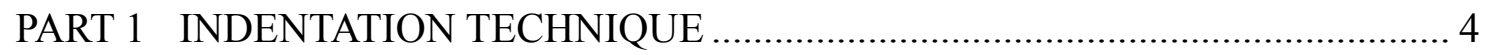

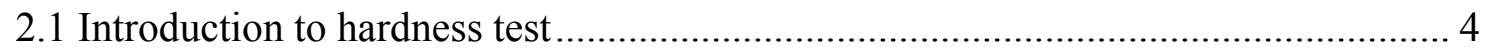

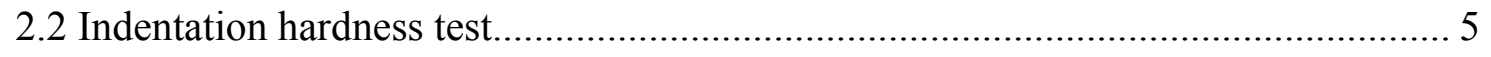

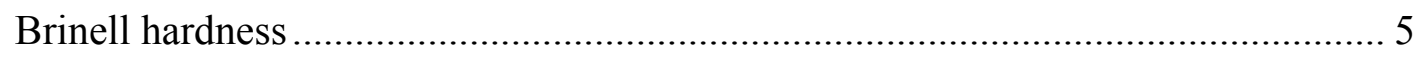

Meyer hardness

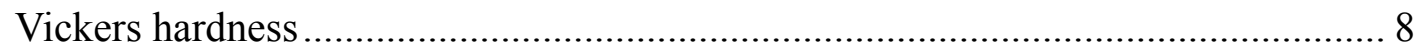

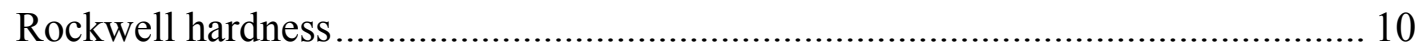

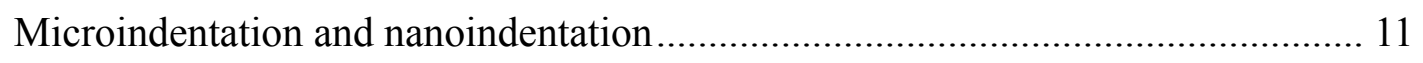

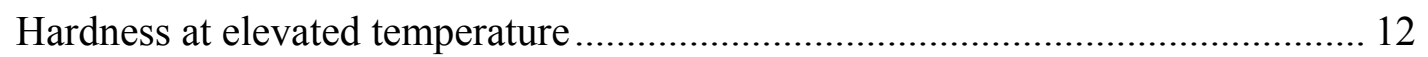

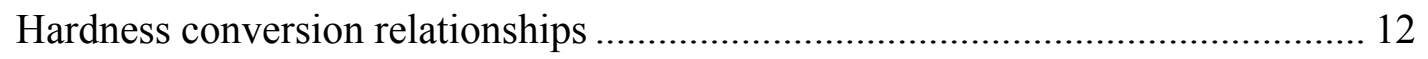

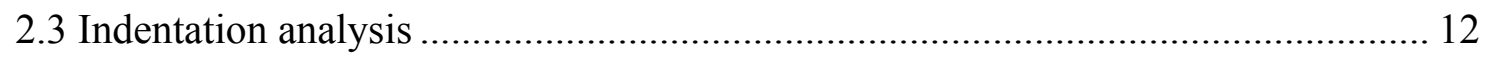

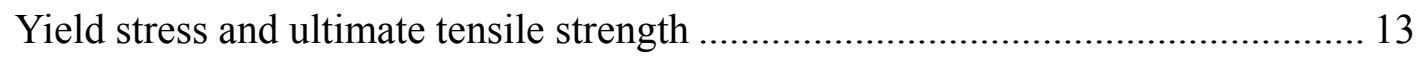

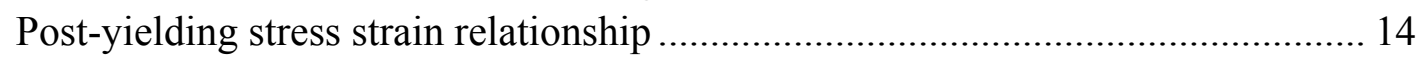

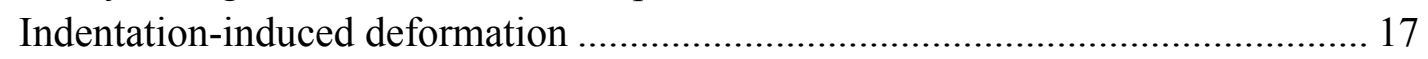

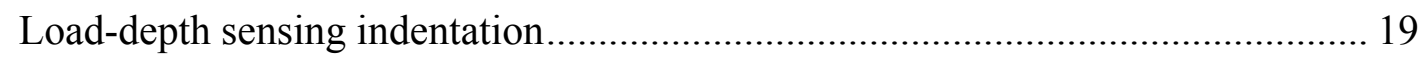

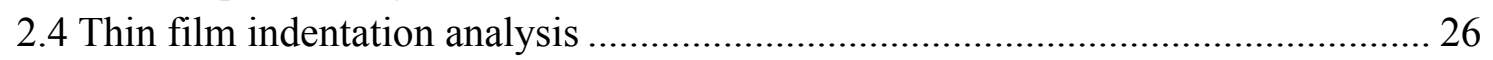

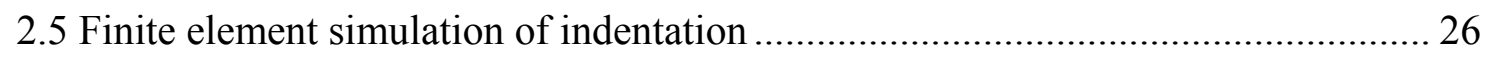

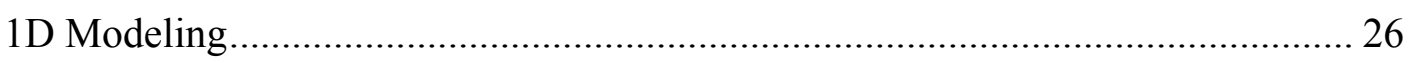

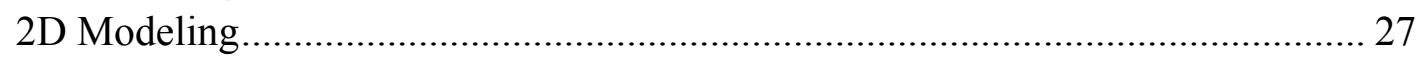

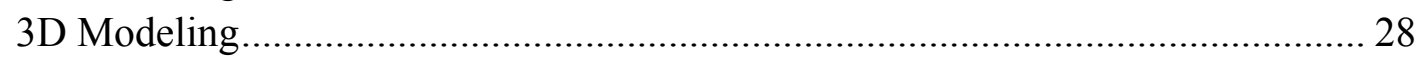

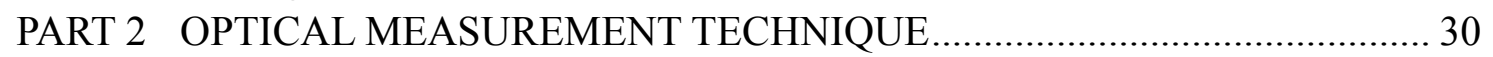

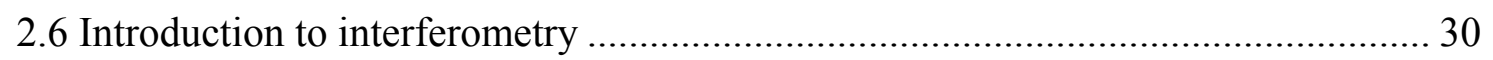

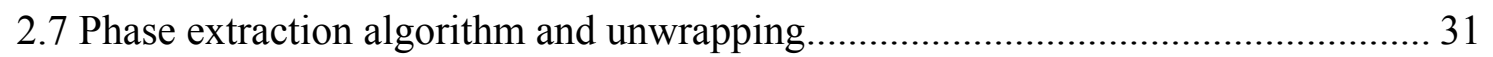

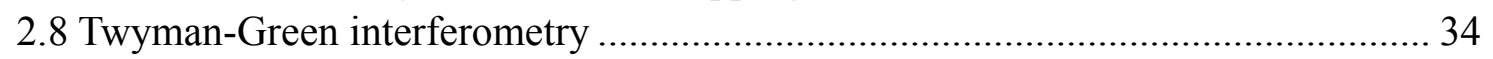

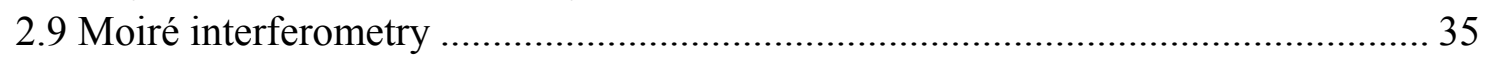

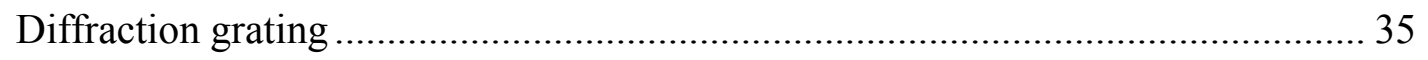

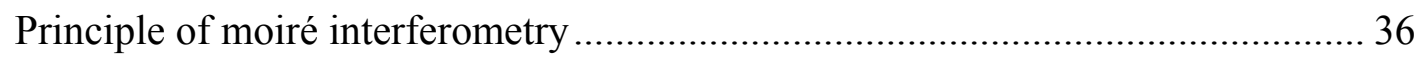

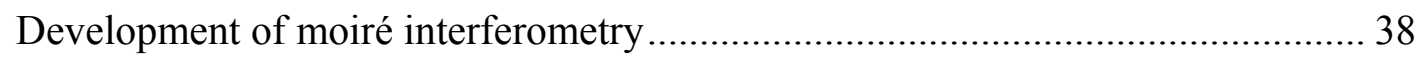


CHAPTER 3 FINITE ELEMENT ANALYSIS ...................................................................40

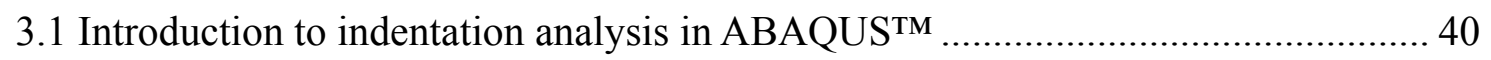

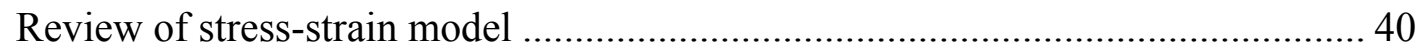

Classical metal elastic-plastic analysis in ABAQUS............................................. 43

Additional considerations for indentation contact modeling.................................... 45

3.2 Finite element models for indentation analyses...................................................... 46

Axisymmetric indentation model ........................................................................ 46

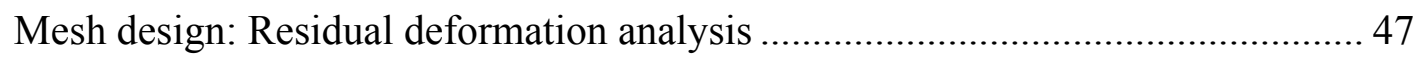

Mesh design: Elastic recovery analysis ............................................................... 48

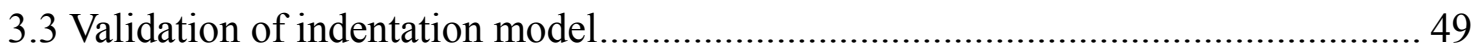

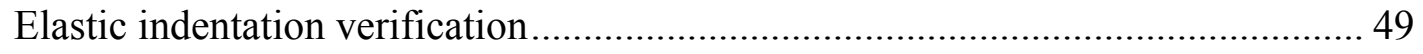

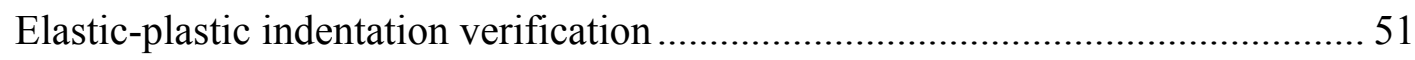

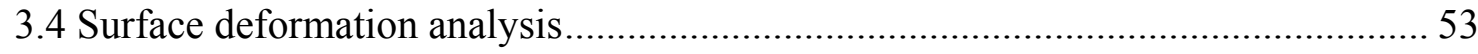

Top free surface stress-strain analysis: Yield strength............................................ 53

Top free surface elastic recovery: Young's modulus ………………….................... 55

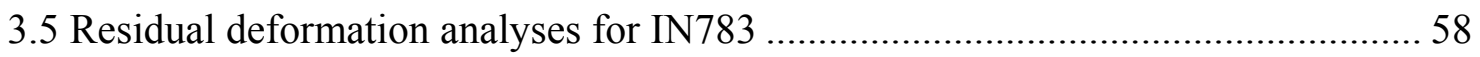

CHAPTER 4 RESIDUAL DEFORMATION ANALYSES..............................................61

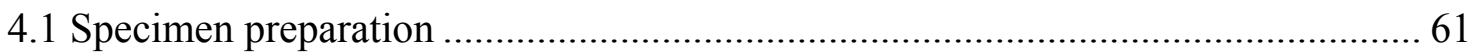

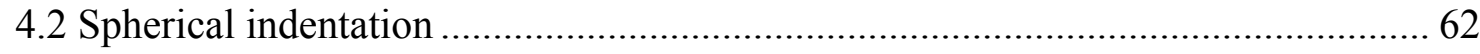

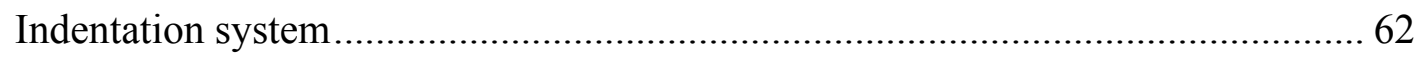

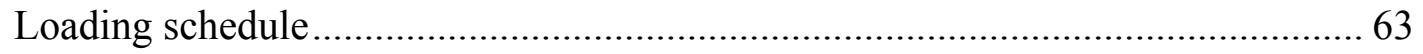

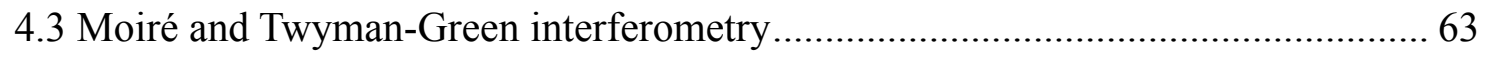

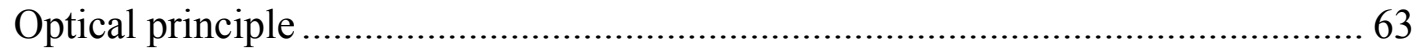

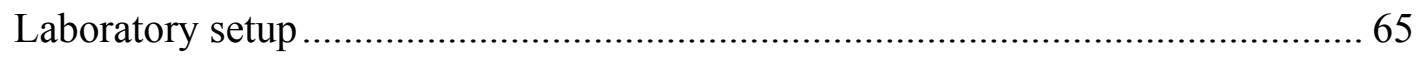

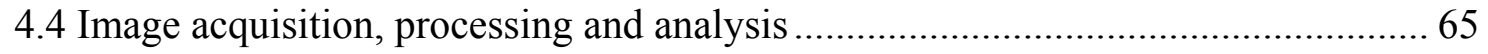

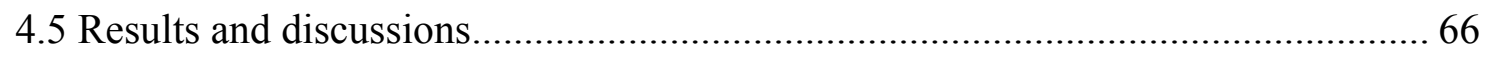

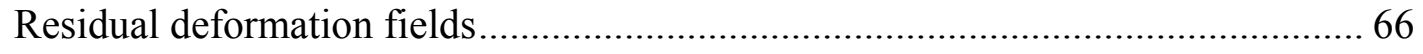

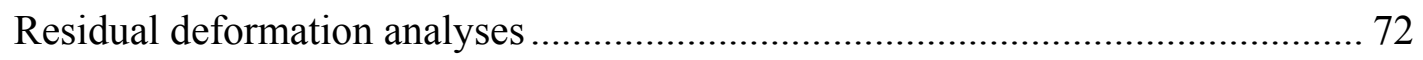

Indentation stress-strain vs. stress-strain curve .................................................. 76

CHAPTER 5 TRANSPARENT INDENTER MEASUREMENT .....................................77

5.1 Through Indenter Measurement (TIM) system ..................................................... 77

Optical schematic of integrated indentation system............................................... 77

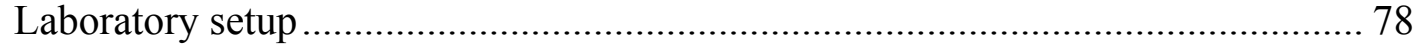

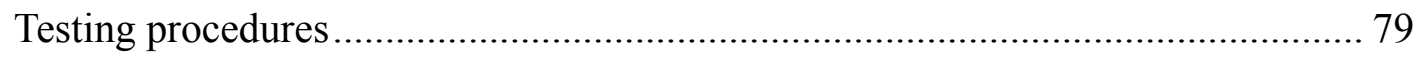

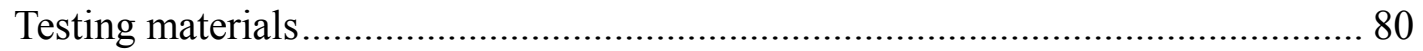

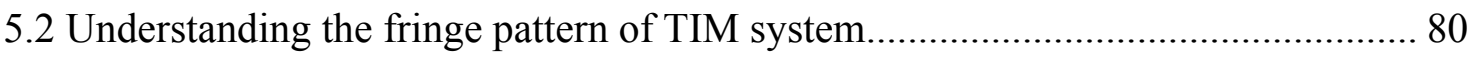

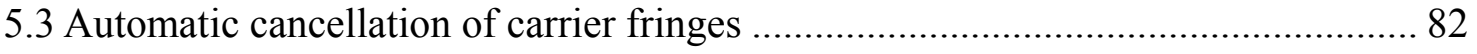

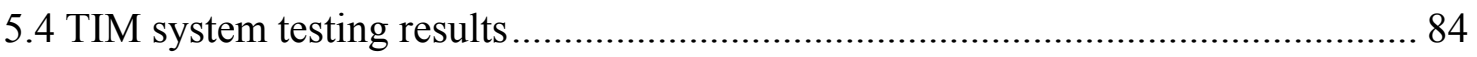

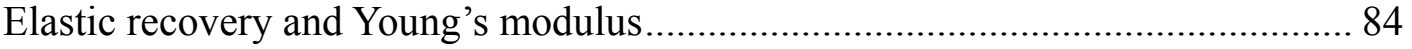

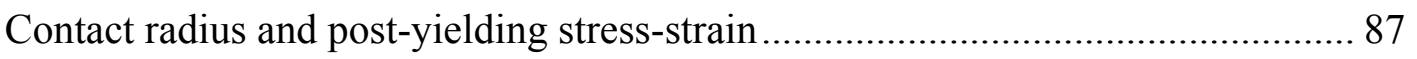

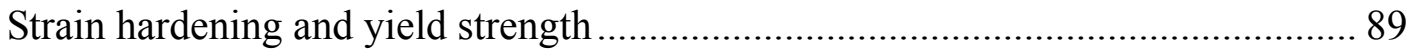


Material surface condition inspection ............................................................ 90

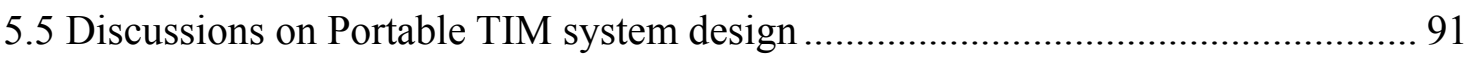

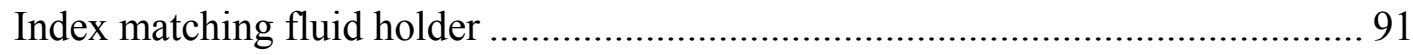

Automatic reference beam blocking...................................................................... 91

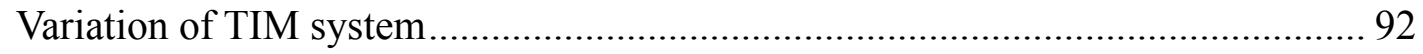

CHAPTER 6 SUMMARY, CONCLUSIONS AND FUTURE PERSPECTIVES .........94

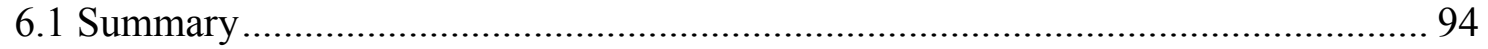

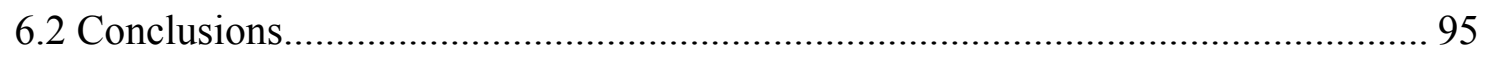

6.3 Recommendations and future perspectives ............................................................ 96

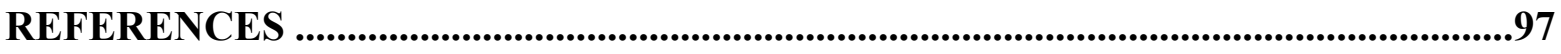

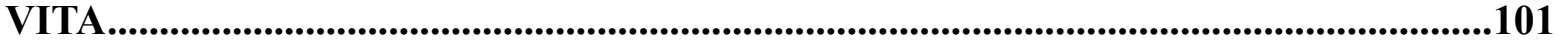




\section{LIST OF FIGURES}

Figure\#

Title

Page\#

Figure 2.1 Basic parameters in Brinell hardness test ........................................... 6

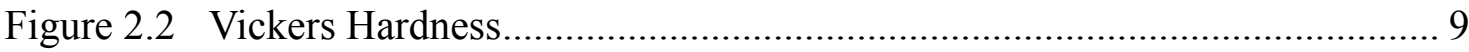

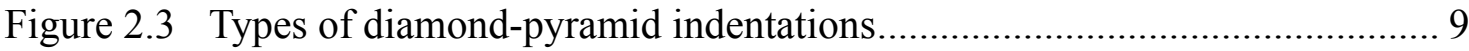

Figure 2.4 Principle of Rockwell hardness test ................................................... 10

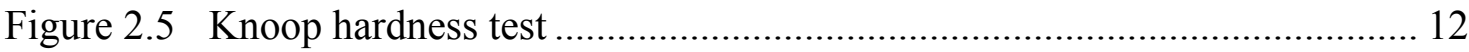

Figure 2.6 Plastic zone under a Brinell indenter ................................................ 13

Figure 2.7 Illustration of radial cracks geometry of Vickers indentation ................... 18

Figure 2.8 Typical indentation load-depth relation ............................................... 19

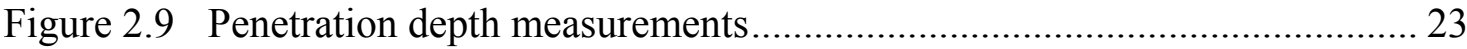

Figure 2.10 Indentation depth definition ........................................................ 24

Figure 2.11 Definition of various quantities used in indentation analyses................. 25

Figure 2.12 Example of constant 4 step phase shifting and phase map .................... 33

Figure 2.13 Unwrapping method and error patterns ............................................ 34

Figure 2.14 Principle of Twyman-Green interferometry .................................... 35

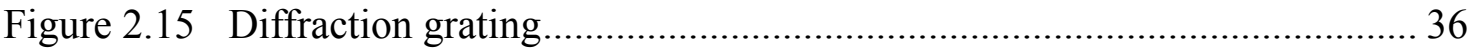

Figure 2.16 WVU Moiré interferometry with automatic phase shifting control........ 39

Figure 2.17 Optical phase shifting implementation by LCVR at WVU ................... 39

Figure 3.1 Ultimate tensile strength and true stress-strain curve ............................ 41

Figure 3.2 Elastic-perfect plastic material used in ABAQUS ................................ 44

Figure 3.3 Elastic linear work hardening material used in ABAQUS ...................... 44

Figure 3.4 Axisymmetric indentation model using a spherical indenter.................... 47

Figure 3.5 Finite element mesh design for residual deformation analyses ................ 48

Figure 3.6 Finite element mesh design for elastic recovery analyses ....................... 49

Figure 3.7 Load-depth curve for elastic Aluminum .............................................. 50

Figure 3.8 Load-depth curve for elastic IN783 .................................................... 50

Figure 3.9 von-Mises stress distribution for elastic IN783 material .......................... 51

Figure 3.10 Comparison of residual deformation between FEA and experimental .... 52

Figure 3.11 Load-depth curve of IN783 with maximum 550N indentation............... 52

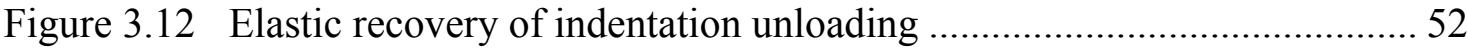

Figure 3.13 Comparison of surface von-Mises stress distribution ............................ 55

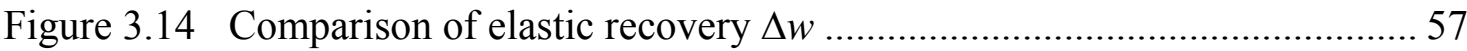

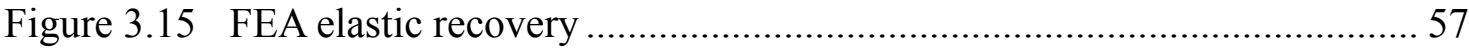

Figure 3.16 Load vs. contact-radius and elastic-plastic boundary relation ................. 58

Figure 3.17 Frictionless FEA results compared with experimental results ................ 59

Figure 3.18 Friction effects on surface residual deformation.................................. 59

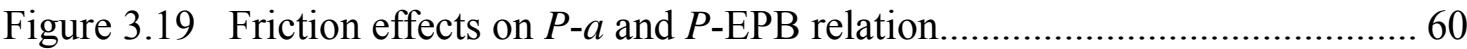




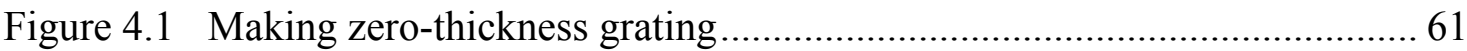

Figure 4.2 Indentation system for residual field measurements............................. 62

Figure 4.3 Combined phase-shifting moiré and Twyman-Green Interferometry ........ 64

Figure 4.4 Configuration of LCVR for combined optical setup ...............................64 64

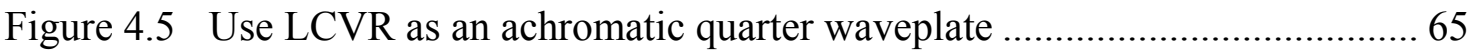

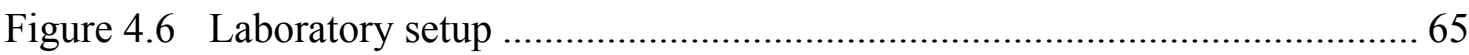

Figure 4.7 IMAQTools software for image acquisition and phase-shifting control.... 66

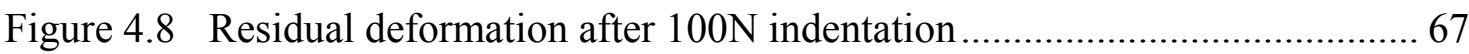

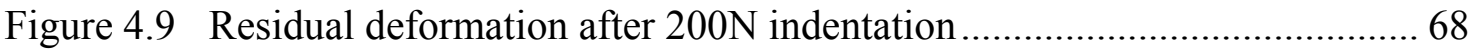

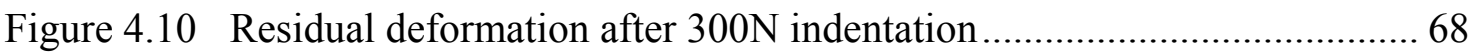

Figure 4.11 Residual deformation after 400N indentation ................................... 69

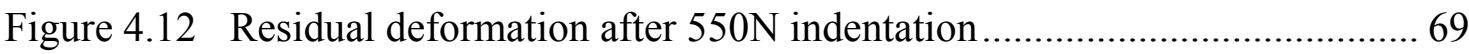

Figure 4.13 Residual deformation after $610 \mathrm{~N}$ indentation ................................... 70

Figure 4.14 Residual deformation after $710 \mathrm{~N}$ indentation ..................................... 70

Figure 4.15 Residual deformation after 820N indentation .......................................... 71

Figure 4.16 Residual deformation after $900 \mathrm{~N}$ indentation ..................................... 71

Figure 4.17 Residual deformation after $1000 \mathrm{~N}$ indentation ................................... 72

Figure 4.18 Identified elastic-plastic boundary (EPB) (1) .................................... 73

Figure 4.19 Identified elastic-plastic boundary (2) ............................................. 73

Figure 4.20 Identified elastic-plastic boundary (3) ................................................. 74

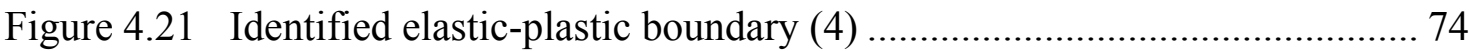

Figure 4.22 Experimental contact radii and elastic-plastic boundaries ..................... 75

Figure 4.23 Determination of yield strength after 550N indentation ....................... 75

Figure 4.24 Results of yield strength (Average: $770 \mathrm{MPa}$ ) and corresponding error... 76

Figure 4.25 Indentation stress-strain vs. true stress-strain of IN783 superalloy ......... 76

Figure 5.1 Schematic optical principle of the TIM system .................................... 78

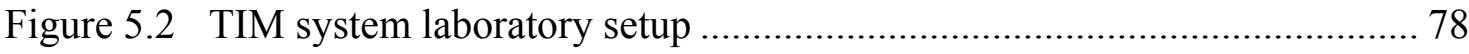

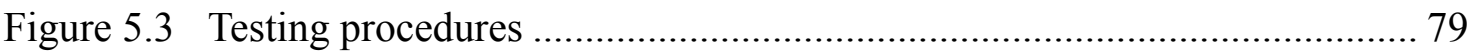

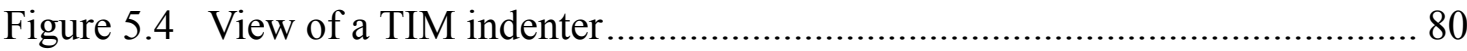

Figure 5.5 Initial fringe pattern in TIM system ................................................. 81

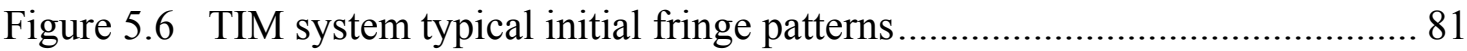

Figure 5.7 Comparison of experimental and theoretical initial fringe patterns........... 82

Figure 5.8 Carrier fringe patterns during loading-unloading .................................. 84

Figure 5.9 TIM system typical fringe patterns, phase and image at loading............. 84

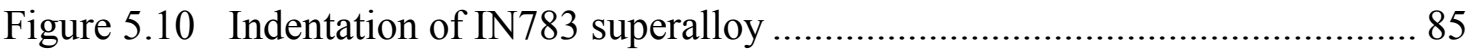

Figure 5.11 Experimental elastic recovery and $\Delta w \sim t$ relation for IN783 ................ 85

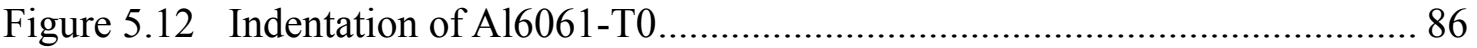

Figure 5.13 Experimental elastic recovery and $\Delta w \sim t$ relation for Al 6061-T0 .......... 86

Figure 5.14 Typical in-situ contact radius measurement for IN783 ........................ 88

Figure 5.15 IN783 post-yielding stress-strain data........................................... 88

Figure 5.16 Typical in-situ contact radius measurement for Al6061-T0.................... 88 
Figure 5.17 Al6061-T0 post-yielding stress-strain data ........................................ 88

Figure 5.18 Calculation of strain hardening coefficient, Log-log plot...................... 89

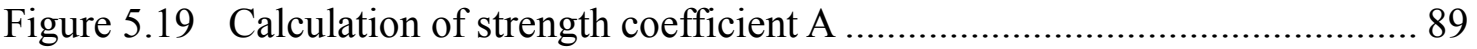

Figure 5.20 Real-time in-situ monitoring of Al2024-T3 with thin layer coating ....... 90

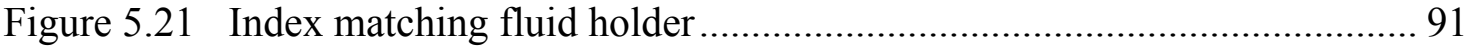

Figure 5.22 Modified TIM system ......................................................................... 92

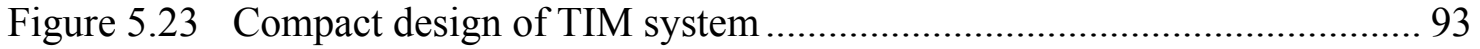




\section{LIST OF TABLES}

Table\#

Title

Page\#

Table 2.1 Moh's hardness scale ................................................................... 5

Table 2.2 Comparison of Meyer index and stress-strain hardening coefficient ........... 8

Table 2.3 Parameters describing power law fits of unloading curves ....................... 25

Table 3.1 Stress and strain conversions for IN783 .............................................. 44

Table 3.2 Material matrix for elastic recovery analyses........................................ 48

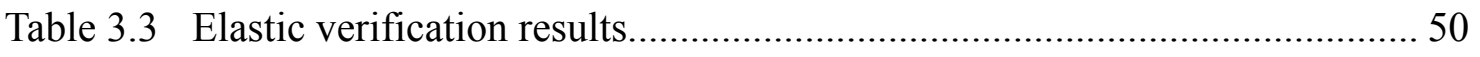

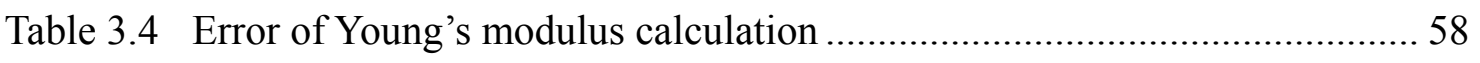

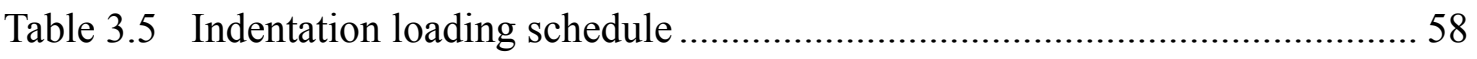

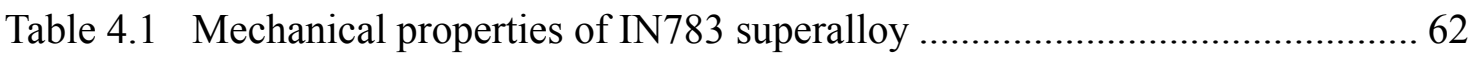

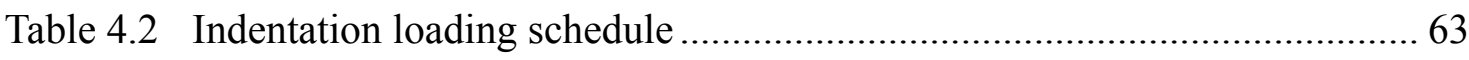

Table 5.1 Experimental error percentage of Young's modulus calculation................ 87 


\section{CHAPTER 1 \\ INTRODUCTION}

\subsection{Problem statement}

Hardness is an important material mechanical property and early studies were mainly focused on the improvement of hardness measurement. Since Tabor ${ }^{[1]}$ systematically studied the indentation hardness tests and showed the application of the spherical indentation approach to obtain material post-yielding true stress-strain curves, the indentation technique has been applied to determine material mechanical properties besides hardness measurement. This technique has been extensively studied and most of the mechanical properties, such as Young's modulus, yield strength, strain hardening coefficient, fracture toughness, ultimate strength, residual stress, creep, coating failure/debonding, strain rate effects etc, have been reported to correlate to indentation hardness tests ${ }^{[1-11,15-47]}$.

However, indentation hardness test is a complicate mechanical process, which involves difficult contact mechanics and nonlinear plasticity deformation. Analytical solutions are difficult to obtain. As a result, much of the understanding of the indentation process has been acquired through experiments and finite element simulations ${ }^{[1-2,5-6,15-47]}$. Thus accurate measurement of indentation parameters is critical in the determination of surface mechanical properties using the indentation technique. For any given indenter, the following parameters may be considered in the study of an indentation test;

1. Contact radius or contact area or projected area.

2. Maximum load, used for hardness calculation.

3. Indentation depth, including maximum depth and residual depth.

4. Load-depth curve, the area under the loading curve represents the total work done by the indentation; the area under the unloading curve represents the recovered elastic strain energy during unloading.

5. Surface deformation ( $u, v$, and $w$ fields), surface stress, strain distribution during or after an indentation test.

6. Sub-surface deformation, internal stress, strain distribution.

7. Strain/Loading rate, for static or quasi-static indentation tests, the strain rate effects are seldom considered.

Among all the indentation parameters, the first four parameters were studied extensively either experimentally or numerically. The technology advances in instrumented indentation have made it possible that force and depth can be recorded continuously as an indentation is made. The indentation load-depth data thus derived can be used to determine mechanical properties even when the indentations are too small to be imaged conveniently. The most direct 
results from these measurements are Young's modulus and post-yielding stress-strain data. The initial unloading stiffness is often used to determine the Young's modulus. If the measurement of contact radius using a microscope is impossible, it can be estimated from the load-depth data. Then the results can be correlated to post-yielding stress-strain data through Tabor's empirical relation. With the development of various thin film materials, depth-sensing indentation is often applied to evaluate mechanical properties of the thin film material.

As discussed shortly in the literature review, ascertaining mechanical properties using spherical indentation technique is still not routine work. In order to obtain post-yielding stress-strain data through a single spherical indentation test, continuous measurement of the most critical variable, contact radius is necessary but still not available. It can only be estimated from the load-depth data. This estimation process involves complicate algorithms and assumptions which are not universally applicable. It can only be applied to certain materials ${ }^{[43]}$. The direct measurement of the contact radius is preferred in this situation, but the traditional indenter blocked the view. So either angled view or through-indenter view may be used for directly measurement of the contact radius.

It is also noticed that the surface deformation is closely related to material mechanical properties. But so far, only the residual fields are discussed ${ }^{[2,6,22,45]}$. The indentation-induced deformation fields are in the order of micrometers. Optical interferometric methods, Moiré and Twyman-Green interferometry are perfect for the measurements of these deformation fields. As of now, there is no explicit link from the deformation fields and mechanical properties. Development of the methodology to bridge the deformation fields and mechanical properties is necessary and will be investigated. 


\subsection{Research objective}

The objective of this research is to develop a new indentation technique, Transparent Indenter Measurement (TIM) method, to ascertain material mechanical properties through accurate indentation parameter measurement.

Optical interferometry, a phase-shifting Twyman-Green interferometer, is incorporated into the indenter head in the development of this new technique, thus it can perform real-time in-situ measurement of indentation-induced out-of-plane deformation and load-contact-radius using a computer controlled data and image acquisition system. The real-time, in-situ high resolution deformation measurement also opens new applications for the indentation technique in the characterization of material surface conditions. The indentation-induced residual deformation fields are also studied using a combined phase-shifting moiré and Twyman-Green interferometer to correlate to material yield strength. During the development of this indentation technique, 2D finite element analyses are conducted to facilitate the development of data analysis procedures. 


\section{CHAPTER 2}

\section{LITERATURE REVIEW}

The literature review is grouped into two parts, the indentation technique and the optical measurement technique. The first part is focused on the indentation hardness test and its development; and the second part is focused on the optical interferometric deformation measurement methods and indentation related studies.

\section{PART 1 INDENTATION TECHNIQUE}

\subsection{Introduction to hardness test}

The hardness of materials has probably long been assessed by the resistance to scratching or cutting. In general, hardness is the material property which implies a resistance to plastic deformation usually by penetration. In mechanics of material testing, hardness is most likely to mean the resistance to the indentation; and to the design engineer it often means an easily measured and specified quantity which indicates something about the strength and heat treatment of metals. The term hardness may also refer to resistance to bending, scratching, abrasion or cutting. However, material hardness is a poorly defined term and is not an intrinsic material property. It has no precise definitions in terms of fundamental units of mass, length and time. Its value is method-related and it has many meanings depending on the applications involved. There are three general types of hardness measurements depending on the manner in which the test is conducted. These are (1) scratch hardness, (2) indentation hardness, and (3) rebound or dynamic hardness. Only indentation hardness is of major engineering interest in the metal industry ${ }^{[2-4]}$.

Scratch hardness is relative hardness. It is the oldest form of hardness and is of primary interest to mineralogists. Relative hardness of minerals can be assessed by reference to the Moh's scale that ranks the ability of materials to resist scratching by another material. As shown in Table 2.1, 10 standard minerals are arranged in the order of their ability to be scratched. The Moh's scale has been used since 1822. However, the relative hardness testing method is limited in practical use, e.g. measuring relative hardness of micro-constituents, and do not provide accurate numeric data or scales particularly for metals and newly developed materials. Moreover, Moh's scale is not well suited for metals since the intervals are not well spaced in the high-hardness range ${ }^{[2-3]}$.

Indentation hardness is widely used in the metal industry. It involves the formation of a permanent indent on the surface of the metal to be examined. Then it measures the depth or area of an indentation left by an indenter of a specific shape, with a specific force applied for a specific time. Brinell, Vickers and Rockwell are the three principal standard methods. 
Depending on the load and indenter geometry, each of these methods is divided into a range of scales, which may be suitable for different material categories.

In dynamic-hardness measurements, the indenter is usually dropped onto the metal surface; then the energy of impact is used to measure the hardness. The Shore Scleroscope, which is the commonest example of a dynamic-hardness tester, measures the hardness in terms of elasticity of the material and the hardness depends on the height to which the indenter rebounds. The harder the material, the higher the rebound. Advantages of this method are the portability and non-marking of the test surface.

Table 2.1 Moh's hardness scale

\begin{tabular}{|r|l|}
\hline Diamond & 10 \\
\hline Corundum & 9 \\
\hline Topaz & 8 \\
\hline Quartz & 7 \\
\hline Orthoclase (Feldspar) & 6 \\
\hline Aptite & 5 \\
\hline Fluorite & 4 \\
\hline Calcite & 3 \\
\hline Gypsum & 2 \\
\hline Talc & 1 \\
\hline
\end{tabular}

\subsection{Indentation hardness test}

The indentation hardness test depicts the plastic deformation and is of great interest in this research. For the macro indentation hardness test, Brinell, Vickers and Rockwell are the three principal standard methods. If loads are under $1 \mathrm{kgf}$, it is usually called microindentation instead of microhardness to avoid confusion. Currently, Vickers, Knoop and Berkovich indenters are frequently used in microindentation tests. For macro indentation, it is also found that over a wide range of experimental conditions the surface roughness has little effect on the size of the indentation. It has practical importance which means high degree of surface finish is not necessary for making satisfactory hardness measurements. But for the microindentation test, the specimen surface usually needs a high quality finish.

\section{Brinell hardness}

The Brinell hardness test is the first widely accepted and standardized indentation hardness test which was proposed by a Swedish engineer, J. A. Brinell in 1900s. It has been widely used in casting and forging.

As shown in Figure 2.1, the Brinell hardness test consists of indenting the metal surface with a $10-\mathrm{mm}$ harden steel or carbide ball subjected to a load of $3000 \mathrm{~kg}(\mathrm{kgf})$. The load may be reduced to 500 or $1500 \mathrm{~kg}$ to avoid excessive indentation for soft materials. The full load is normally applied for 10 to 15 seconds in the case of iron and steel and for at least 30 seconds in 
the case of other metals ${ }^{[3]}$. The residual diameter of the indentation is measured using a low-power microscope and the average of at least two readings at right angles should be used to minimize the error. Then Brinell hardness number $(\mathrm{BHN})$ is calculated based on contact area (Spherical cap) of the impression using Equation (2-1)

$$
B H N=\frac{P}{\pi \frac{D}{2}\left(D-\sqrt{D^{2}-d^{2}}\right)}=\frac{P}{\pi D t}
$$

Where $P$ is the applied load in $\mathrm{kg}, D$ is the diameter of the indenter ball in $m m, d$ is the diameter of the contact area in $\mathrm{mm}, \mathrm{t}$ is the depth of the impression in $\mathrm{mm}$.

Note: $1 \mathrm{~kg} / \mathrm{mm}^{2}=1 \mathrm{kgf} / \mathrm{mm}^{2}=9.8 \mathrm{MPa}$

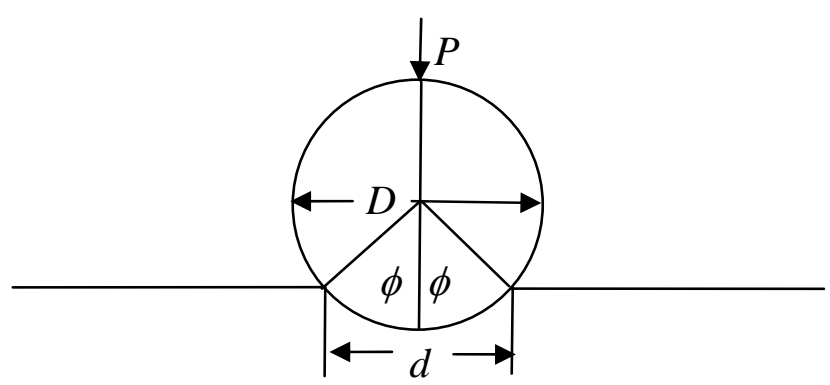

Figure 2.1 Basic parameters in Brinell hardness test

Since the Brinell hardness number vary with load, it should reveal the testing conditions (i.e. ball diameter, load and holding time). For a Brinell hardness test, the preferable d/D lies $0.25 \sim 0.50$. It is impossible to cover the entire range of hardness encountered in all commercial metals with a single load. For a non-standard load or non standard ball test, it is necessary to produce geometrically similar indentation test, which is achieved as long as the included angle $2 \phi$ remains constant. Equation (2-1) can be re-written as ${ }^{[2]}$,

$$
B H N=\frac{P}{\frac{\pi}{2} D^{2}(1-\cos \phi)}
$$

$2 \phi$ remaining constant implies,

$$
\frac{P_{1}}{D_{1}{ }^{2}}=\frac{P_{2}}{D_{2}{ }^{2}}=\frac{P_{3}}{D_{3}{ }^{2}}
$$

Compared to the other hardness testing methods, the Brinell ball makes the deepest and widest indentation, so it is less influenced by surface scratches and roughness than other hardness tests, which makes it best for achieving the bulk or macro-hardness of a material, particularly for those materials with heterogeneous structures. On the other hand, this may preclude the use of this test with small objects or in critically stressed parts where the indentation can be a potential site of failure. 


\section{Meyer hardness}

In practice, Meyer hardness is rarely used, but it is less sensitive to the applied load than Brinell hardness. It is originated with Meyer in 1908. He suggested using the projected area of the impression rather than the surface area used in Brinell hardness. Finally Meyer hardness ended with a more fundamental measure of indentation hardness since it reflects the mean pressure $^{[1-2]}$.

$$
\text { Meyer Hardness }=p_{m}=\frac{P}{\pi r^{2}}=\frac{4 P}{\pi d^{2}}
$$

The quasi-empirical relation, Meyer's law plays an important role in determination of material mechanical properties using indentation technique. It correlates the load and the size of the indentation in a power law form. For a fixed diameter ball, it is written as,

$$
P=k d^{n^{\prime}}, \quad P=k d^{n^{\prime}} \approx k d^{n+2}
$$

Where $P=$ applied load in $\mathrm{kg}, d=$ diameter of indented area in $\mathrm{mm}$

$n '=$ a material constant related to strain hardening of metal,

$k=\mathrm{a}$ material constant expressing resistance of metal to penetration

The Meyer's index $n$ ' is related to strain hardening coefficient $n$ approximately by the equation $n^{\prime} \approx n+2$ as shown in Table 2.2 . It should be noted that this is only approximately accurate.

With different ball size, Meyer also found experimentally the following relation stands,

$$
\frac{P}{D^{2}}=A\left(\frac{d}{D}\right)^{n^{\prime}}=A\left(\frac{d}{D}\right)^{n+2}
$$

Equation (2-6) also indicates that the geometrically similarity requires $P / D^{2}$ remains constant. For a geometrically similar indentation, the Meyer hardness (or the Brinell hardness) will get the same hardness number for different indentations. This conclusion is widely used in practical hardness measurements ${ }^{[1]}$.

Since $n$ is a constant for any given material, this can also be written as ${ }^{[6]}$,

$$
P=D^{2} A\left(\frac{d}{D}\right)^{n^{\prime}}=D^{2} A\left(\frac{2 a}{D}\right)^{n+2}=2^{n+2} A \frac{a^{n+2}}{D^{n}}=k \frac{a^{n+2}}{D^{n}}
$$

More general form can be written as ${ }^{[1]}$,

$$
\frac{P}{D^{2}}=f(d / D)
$$

Equation (2-8) is a general form of Tabor's relation which will be discussed later.

It should be noted that Meyer's law is only valid when deformation around the indenter is fully plastic, in which the plastic zone approaches the specimen surface and the boundary is 
larger than the contact radius. So there is a low load limit below which Meyer's law is not valid $^{[1]}$. Usually the indenter ball needs to submerge almost to their diameters to make a large indentation.

Table 2.2 Comparison of Meyer index and stress-strain hardening coefficient

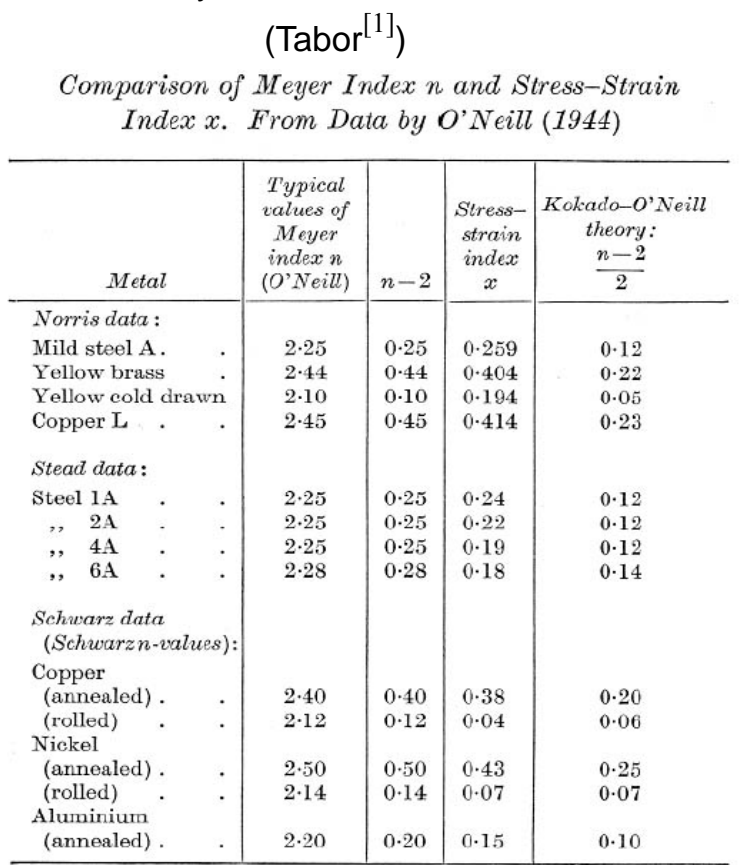

\section{Vickers hardness}

The Vickers hardness test method consists of indenting the test material with a diamond indenter subjected to a load of 1 to $120 \mathrm{kgf}$. The full load is normally applied for 10 to 15 seconds. After removal of the load, the Vickers Diamond Pyramid Hardness (DPH) is calculated using maximum load divided by contact area (Equation 2-9). The average of the two diagonal lengths is usually used to minimize the error ${ }^{[2-3]}$.

$$
\begin{aligned}
& H V=\frac{2 P \sin (\theta / 2)}{d^{2}}=\frac{1.854 P}{d^{2}} \quad\left(\mathrm{kgf} / \mathrm{mm}^{2}\right) \\
& \text { Where } \begin{array}{r}
P=\text { applied load, } \mathrm{kg} \\
d=\text { average length of diagonals } d_{1} \text { and } d_{2},\left(d_{1}+d_{2}\right) / 2, \mathrm{~mm} \\
\theta=\text { angle between opposite faces of diamond }=136^{\circ}
\end{array}
\end{aligned}
$$

As shown in Figure 2.2, the indenter is a square-base diamond pyramid of which the included angle between opposite faces is $136^{\circ}$, and the depth of indentation is about $1 / 7$ of the diagonal length. This angle was chosen to approximate the most desirable ratio of indentation diameter to the ball diameter in Brinell hardness test (For the Brinell indentation, let $d / D=$ 0.375 , which results in a $136^{\circ}$ cone angle). As long as the Brinell hardness test is of normal depth $(d / D$ lies $0.25 \sim 0.5)$, the DPH and the BHN hardness are nearly identical.

The continuous scale of Vickers hardness makes it widely accepted for research work. On 
the other hand, Brinell or Rockwell hardness tests usually need to change either the load or the indenter at some point in the hardness scale. More important, impressions by this pyramid indenter are always geometrically similar, so the hardness is independent of the load. This is true except at very light load.
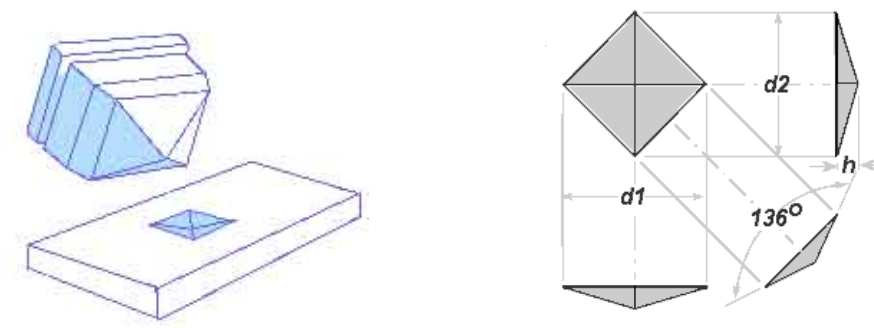

Figure 2.2 Vickers Hardness

(Newage Testing Instruments, Inc and http://gordonengland.co.uk/hardness/ ${ }^{[3-4]}$ )

It is inconvenient for a routine test since the Vickers hardness test needs careful surface preparation and direct impression diagonal length measurements using a microscope. Recent development of portable Vickers hardness tester, such as the TIV(Through Indenter Viewing $)^{[5]}$ has gained popularity of using Vickers hardness testing for on-site loading status hardness measurement of various engineering material. The live indentation picture displayed on the instrument's LCD allows immediate characterization of the quality of the Vickers diamond indentation. By directly viewing through the diamond indenter under load, Through Diamond Technique ${ }^{[5]}$ opens up new applications for mobile hardness testing not only to coils, thin layers and coatings but also to different materials like plastics, glass and high-tech materials like ceramics or intermetallics.

Anomalies (piling-up or sinking-in) are frequently observed in the Vickers indentation as shown in Figure 2.3. When sinking-in or piling-up happens, an empirical correction is often needed to insure the accurate measurement of diagonal length. By studying the effects of strain hardening coefficient, Alcala et $\mathrm{al}^{[6]}$ concluded that sinking-in predominates in materials where strain hardening coefficient $n>0.2$.

(a)

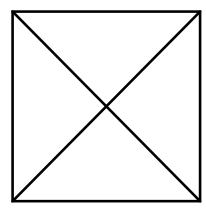

(b)

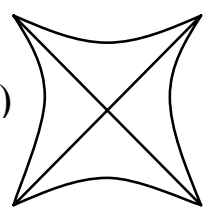

(c)

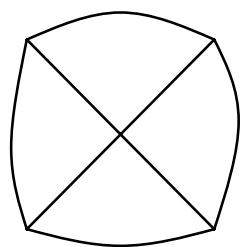

(a) Perfect indentation; (b) Pin-cushion indentation due to sinking in;

(c) Barreled indentation due to ridging

Figure 2.3 Types of diamond-pyramid indentations

(George E. Dieter ${ }^{[2]}$ ) 


\section{Rockwell hardness}

The Rockwell hardness test is most widely used in the United States. It consists of indenting the tested material with a diamond cone or hardened steel ball indenter. This hardness test has many different scales. The details are defined in ASTM standard E18-74 ${ }^{[7]}$. The most commonly used indenters are $120^{\circ}$ diamond cones (Brale indenter with a slightly rounded point) and $1.6 \mathrm{~mm}\left(1 / 16^{\prime \prime}\right)$ or $3.2 \mathrm{~mm}\left(1 / 8^{\prime \prime}\right)$ steel balls, e.g. C scale uses diamond cone indenter and $150 \mathrm{~kg}$ major, B scale uses $1.6 \mathrm{~mm}$ steel ball and $100 \mathrm{~kg}$ major load.

The Rockwell hardness number uses a pre-defined scale which is converted using the penetration depth under constant load. As shown in Figure 2.4, a minor load F0 usually 10kgf is first applied to minimize the amount of surface preparation needed and reduces the tendency for ridging or sinking-in by the indenter. When equilibrium has been reached, an indicating device, which follows the movements of the indenter, is set to a datum position (or reset). A major load $(60,100$ or $150 \mathrm{kgf})$ is then applied while the minor load is still applied. When equilibrium has again been reached, the major load is removed but the minor load is maintained. Removal of the additional major load allows a partial recovery, so reducing the depth of penetration. The permanent increase in depth of penetration, resulting from the major load, is used to calculate the Rockwell hardness number as shown in Equation (2-10). The Rockwell superficial hardness test uses the same principle but with smaller minor and major load. In practices, most testers use dial gage to automatically record the Rockwell hardness number.

$\mathrm{HR}=\mathrm{E}-\mathrm{e}$

Where $\mathrm{E}=\mathrm{a}$ constant depending on the form of indenter:

100 units for diamond indenter, 130 units for steel ball indenter

$\mathrm{e}=$ permanent increase in depth of penetration due to major load F1 measured in units of $0.002 \mathrm{~mm}(0.00008 \mathrm{in})$

The Rockwell hardness test is fast (directly read out hardness number), free from personal error, and is able to distinguish small hardness and has small impression size. It can be considered as a non-destructive test. Disadvantages include many arbitrary non-related scales and possible effects from the specimen support anvil.

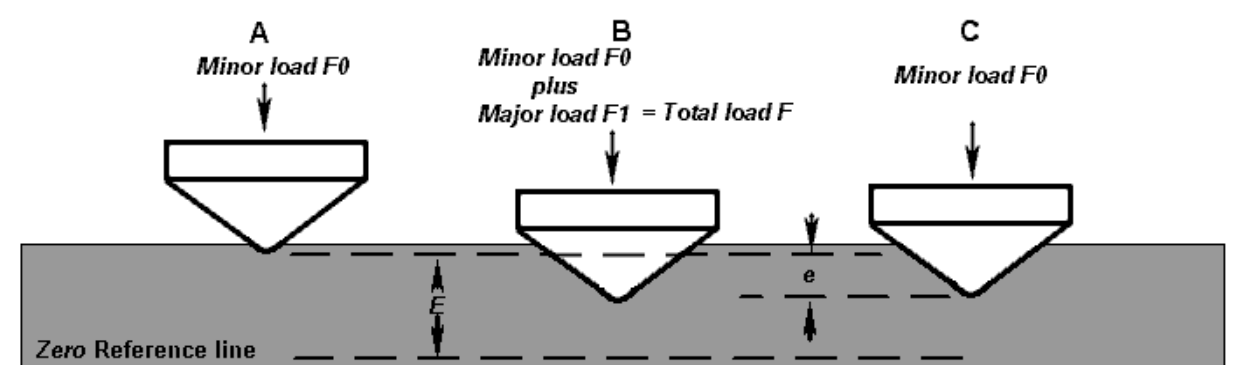

Figure 2.4 Principle of Rockwell hardness test (http://gordonengland.co.uk/hardness/ ${ }^{[3]}$ ) 


\section{Microindentation and nanoindentation}

For loads not exceeding $1 \mathrm{kgf}$, the indentation hardness is often called microindentation instead of microhardness to avoid confusion. Nanoindentation refers to a hardness test with a load less than $1 \mathrm{~N}$ and the size of the indentation is in the nanometer scale. Different from the macroindentation, diamond indenters, such as Vickers diamond pyramid, Knoop elongated diamond pyramid, Berkovich triangle-based diamond pyramid and diamond cone are often used to produce the hardness test. As a powerful tool to study the mechanical properties of components at service condition and thin-film material, both microindentation and nanoindentation gained more applications recently.

The testing procedure is very similar to that of the standard Vickers hardness test; except that it is done on a microscopic scale with higher precision instruments and the surface being tested generally requires a metallographic finish. For both microindentation and nanoindentation, considerable care and experience are necessary to obtain good accuracy. For microindentation, the size of the impression is in the order of micrometers, and precision microscopes are needed. While for nanoindentation, AFM can be equipped to measure the impression. With the development of nanoindentation, indirect measurement of hardness through load-depth sensing technique is also developed. For very light load, nanoindentation is found to be size-dependent. Theories have been developed to study this size-dependent effect, such as mechanism-based strain gradient (MSG) plasticity ${ }^{[11]}$ which uses the concept of the length scale to bridge the difference.

The Knoop indenter, developed at the Nation Bureau of Standards, has been widely used in microindentation and nanoindentation. As shown in Figure 2.5, it is an elongated diamond pyramid indenter and the ratio between long and short diagonals is about 7:1. Only the longest diagonal of the indentation is used to calculate the Knoop hardness number $(\boldsymbol{K H} N)$ as described in Equation (2-11). The depth of the Knoop impression is about 1/30 of its length. The special shape of the Knoop indenter makes it possible to place indentations much closer together than Vickers. This is very useful to measure steep hardness gradient. Also the small indented area compared with Vickers makes it very useful for thin layer measurements.

$$
K H N=\frac{P}{A}=\frac{P}{C L^{2}}
$$

Where $P=$ applied load, $k g$

$A=$ un-recovered projected area of the indentation, $\mathrm{mm}^{2}$

$L=$ measured length of long diagonal of indentation, $\mathrm{mm}$

$C=$ a constant for each indenter supplied by manufacturer $(\sim 0.07028)$. 

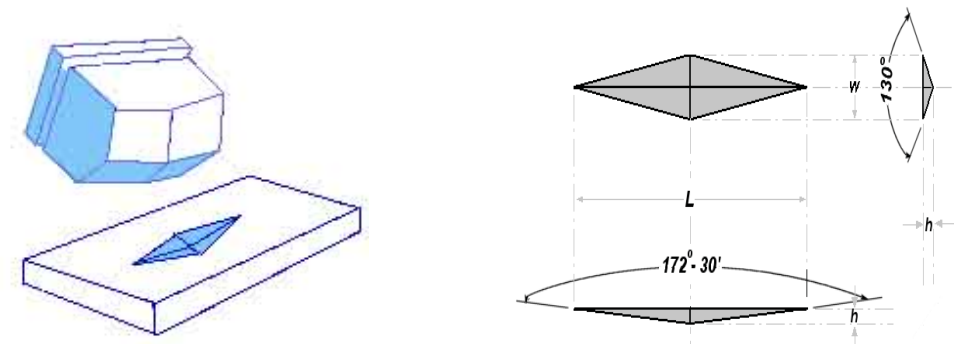

Figure 2.5 Knoop hardness test (http://gordonengland.co.uk/hardness/ \& $\underline{\text { http://www.hardnesstesters.com }}{ }^{[3-4]}$ )

\section{Hardness at elevated temperature}

It is possible to measure the hardness at elevated temperatures with different indenters. Certain degree of success has been obtained in correlating hot hardness with high temperature strength properties. The hot hardness gives a good indication of the potential usefulness of an alloy for high temperature applications. Westbrook ${ }^{[8]}$ showed temperature dependence of hardness can be expressed by,

$$
H=A e^{-B T}
$$

Where $H$ is Hardness in $\mathrm{kg} / \mathrm{mm}^{2} ; T$ is testing temperature in $\mathrm{K} ; A$ and $B$ are constants.

\section{Hardness conversion relationships}

From a practical point of view, it is important to be able to convert the results among different hardness tests. Since hardness is not an intrinsic material property and the conversion between different methods and scales cannot be made mathematically exact for a wide range of materials, no universal hardness conversion relationships had been developed. All the conversions are empirical relationships.

\subsection{Indentation analysis}

Indentation tests are complex processes, which involve contact mechanics, material nonlinearity and even fracture mechanics. Analytical solutions are very difficult to obtain for general cases. As a result, much of the understanding of the indentation process has been acquired through experiments and finite element simulations. How to analyze the indentation parameters and correlate these parameters to mechanical properties were and will continue to be a challenge to the researcher.

The elastic contact problem is first studied by Hertz ${ }^{[15]}$ in the later $19^{\text {th }}$ century, in which he tried to find a precise definition of hardness through the elastic process. Later Boussinesq ${ }^{[15]}$ developed a method based on the potential theory to compute the stresses and displacements in an elastic body loaded by any rigid, axisymmetric indenters. For indentations involved plastic deformation, early studies were focused on the yield strength and ultimate tensile strength. 
Since Tabor ${ }^{[1]}$ showed the application of the spherical indentation approach to obtain the post-yielding stress strain relationship, it is realized that the indentation technique can be used as a mechanical probe. Based on the measured parameters, most of the mechanical properties have been investigated such as residual stress, Young's modulus, post-yielding stress-strain data, fracture toughness etc.

\section{Yield stress and ultimate tensile strength}

The mean compressive stress required to cause plastic flow in the indentation hardness test will exceed that in a simple compression test because of the surrounded elastic material, which acts to hinder plastic flow. The prediction of that load is a classic problem in plasticity. Prandtl applied slip-line field theory to a flat punch and showed that the constraint factor for plane-strain compression is 2.57 , that is

$$
\frac{p_{m}}{\sigma}=1+\frac{\pi}{2}=2.57
$$

Shaw and DeSalvo (1970) ${ }^{[9]}$ showed the plastic zone under the ball (blunt) indenter is similar to an elastic-plastic boundary resembling the line of constant maximum shear stress beneath a sphere pressed against a flat surface as shown in Figure 2.6. This can be obtained by applying Hertz's theory of contact stresses. The elastic-perfect-plastic analysis results in a constraint factor of $\mathrm{C}=3.0$ for indentation with a spherical ball (1988) as shown in Equation $(2-14)^{[2]}$. It is different from Tabor's experimental results $(\mathrm{C}=2.8)$ for work-hardened material.

$$
\frac{p_{m}}{\sigma_{0}}=C=3.0 \text { or } \sigma_{0}=\frac{p_{m}}{3}
$$

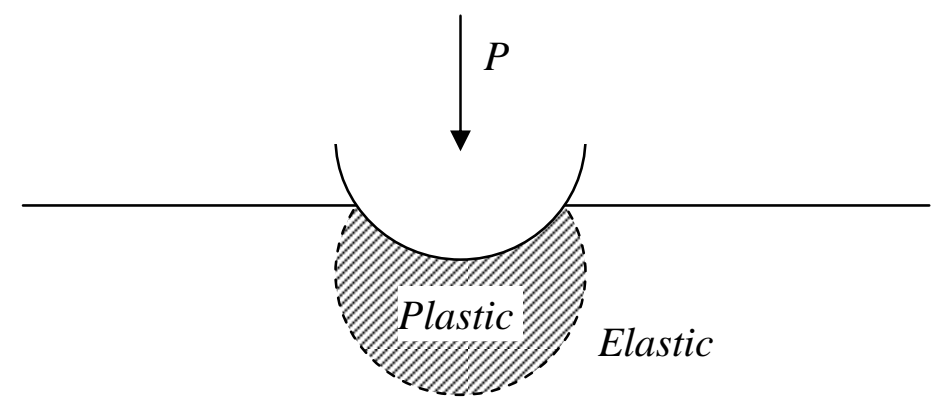

Figure 2.6 Plastic zone under a Brinell indenter

$$
\text { (After Shaw and DeSalvo }{ }^{[9]} \text {, Duplicate from }{ }^{[2]} \text { ) }
$$

According to Cahoon et $\mathrm{al}^{[29]}$, the $0.2 \%$ offset yield stress can be determined with good precision from Vickers diamond hardness (DPH) number by the following relation,

$$
\sigma_{0}=\frac{D P H}{3}(0.1)^{n^{\prime}-2}=\frac{D P H}{3}(0.1)^{n}
$$

Where $\sigma_{0}$ is the $0.2 \%$ offset yield strength in $\mathrm{kg} / \mathrm{mm}^{2}, n^{\prime}$ is Meyer's index, $n$ ' $=n+2$. 
Theoretical analyses of the indentation of elastoplastic isotropic materials without work hardening have been presented by K. L. Johnson (1970) ${ }^{[19]}$ and S. S. Chiang (1982 ${ }^{[33]}$. The central notion of their work is that the extent of plasticity, manifested in the plastic zone volume $\mathrm{V}$, is dictated by the work of indentation, because the plastic volume is the product of the mean contact pressure $\mathrm{H}$ and the indentation volume $\Delta \mathrm{V}$; the plastic zone volume is defined by $\mathrm{H}$ and $\Delta \mathrm{V}$. Johnson and Chiang both showed the ratio $\beta=(V / \Delta V)^{1 / 3}=b / a$ is an important parameter and found the following relations;

$$
\begin{aligned}
& \frac{H}{Y}=\frac{2}{3}\left[1+\ln (\beta)^{3}\right] \\
& \frac{E}{Y} \tan \left(\frac{\pi}{2}-\varphi\right)=6(1-v)\left(\frac{b}{a}\right)^{3}-4(1-2 v)
\end{aligned}
$$

Where $b$ is the plastic zone dimension, it can be verified by dislocation observation; $a$ is the core radius, for Vickers indenter, $\mathrm{a}=\mathrm{D} / \sqrt{2 \pi}$.

R. F. Bishop et al (1945 and 1967) ${ }^{[34-35]}$ introduced work hardening in the indentation theory. They supposed that the limit of flow stress in compression is given by a relation of the following form,

$\sigma=Y+Z \ln \varepsilon$

Where $Z$ is the work-hardening rate and $\varepsilon$ is the deformation.

Their theory leads to the following relation,

$$
\frac{H}{Y}=\frac{2}{3}\left[1+\ln \left(\frac{2 E}{3 Y}\right)\right]+\frac{2 \pi^{2} Z}{27 Y}
$$

For heat-treated plain-carbon and medium-alloy steels, the strain-hardening is almost negligible, so the Ultimate Tensile Strength (UTS) is approximately equal to the yield stress,

$$
\text { UTS }(\text { in } \mathrm{MPa})=3.4 \times(\mathrm{BHN})
$$

\section{Post-yielding stress strain relationship}

Post-yielding stress-strain data can be obtained using the well-known Tabor's empirical relation $^{[1]}$ as shown in Equation (2-20) and Equation (2-21). It was noticed that Meyer hardness with increased plastic strain (Meyer hardness vs. $d / D$ ) is similar to the flow curve. The results showed good agreement for mild steel and annealed copper by introducing indentation strain $(0.2 \mathrm{~d} / \mathrm{D})$. Tabor concluded that true strain and true stress are proportional to the indentation strain and Meyer hardness, respectively. Thus, it is possible to approximate the stress-strain curve by measuring Meyer hardness under the condition of full plasticity such that $d / D$ varies from the smallest value up to largest values through Equation (2-20).. Tabor's approach is basically empirical since the complex stress distribution around the hardness indentation precludes a straightforward relationship. But it should be of interest as means of 
obtaining flow data in situations where it is impossible to measure.

$$
\begin{aligned}
& \varepsilon=0.2 \frac{d}{D} \\
& \sigma_{c y} \approx \frac{P_{m}}{2.8} \\
& \sigma_{c y} \text { is the equivalent true stress }
\end{aligned}
$$

This approach is further developed by Haggag ${ }^{[43-44]}$, Taljat ${ }^{[48]}$, Lee $^{[49]}$, Francis ${ }^{[50]}, \mathrm{Au}^{[51]}$. More sophisticated relations have been developed to correlate indentation hardness to true stress when the load is not large enough to cause full plasticity deformation, but indentation strain defined by Tabor is seldom changed.

Initial flow stress has been solved using elastic indentation analysis,

$$
P_{m}=1.07 \sigma_{t}
$$

For flow stress after initiation and prior to development of a fully-plasticity, the constraint factor has been development to correlate the mean pressure and flow stress ${ }^{[50]}$,

$$
\sigma_{t}=\frac{P_{m}}{C}
$$

Where $C=\left\{\begin{array}{ll}1.07 & \text { for } \phi \leq 1 \\ 1.07+0.53 \ln \phi & \text { for } 1<\phi<26.2 \\ 2.8 & \text { for } \phi \geq 26.2\end{array} \quad, \quad \phi=\frac{\varepsilon_{p} E_{2}}{0.43 \sigma_{t}}\right.$

$E_{2}$ is the specimen elastic modulus,

$\varepsilon_{p}$ is the true plastic strain associated with the indentation.

By using load-depth sensing indentation, Au et al ${ }^{[51]}$ developed a method to estimate the contact radius, but more practical application has been developed by Haggag ${ }^{[43-33]}$. In Haggag's Automated Ball Indentation in the Field Indentation Microscope, multiple loading-unloadings are used to estimate the contact radius, thus avoid the directly measurement of the indent. It is assumed that the homogenous plastic flow portion of the true-stress $\left(\sigma_{t}\right)$ true-plastic-strain $\left(\varepsilon_{p}\right)$ is represented by a power law equation:

$$
\sigma_{t}=K \varepsilon_{p}^{n}
$$

Where $n$ is strain-hardening exponent and $K$ is Strength coefficient

The true plastic strain was then calculated using a modified Tabor's empirical relation,

$$
\varepsilon_{p}=0.2 \frac{d_{p}}{D}
$$

and 


$$
\begin{aligned}
& \sigma_{t}=\frac{4 P}{\pi d_{p}^{2} \delta} \\
& \text { where } d_{p}=\sqrt{0.5 C D\left[h_{p}{ }^{2}+\left(d_{p} / 2\right)^{2}\right] /\left[h_{p}{ }^{2}+\left(d_{p} / 2\right)^{2}-h_{p} D\right]} \\
& C=5.47 P\left(1 / E_{1}+1 / E_{2}\right) \\
& \delta=\left\{\begin{array}{c}
1.12 \ldots \ldots \ldots \ldots . . \phi \leq 1 \\
1.12+\tau \ln \phi \ldots \ldots \ldots \ldots . .1<\phi \leq 27 \\
\delta_{\max } \ldots \ldots \ldots \ldots \ldots \ldots . . \phi
\end{array}\right. \\
& \phi=\varepsilon_{p} E_{2} / 0.43 \sigma_{t} \\
& \delta_{\max }=2.87 a_{m} \\
& \tau=\left(\delta_{\max }-1.12\right) / \ln (27)
\end{aligned}
$$

Where $d_{\mathrm{p}}$ is the plastic indentation diameter, $D$ is the indenter ball diameter, $h_{\mathrm{p}}$ is the plastic indentation depth, $P$ is the applied load, $E_{1}$ is the indenter elastic modulus, $E_{2}$ is the specimen elastic modulus, $\delta$ 's value depends on the stage of development of the plastic zone beneath the indenter, $a_{m}$ is a parameter proportional to the strain rate sensitivity of the test specimen. Use $\sigma_{\mathrm{t}}-\varepsilon_{\mathrm{p}}$ data to fit all the parameters for power law.

It is worth to mention that in the ABI test, yield strength is estimated using different approach. By defining a total indentation diameter $\left(d_{\mathrm{t}}\right)$ using total penetration depth $\left(h_{\mathrm{t}}\right)$ as described in Equation (2-27) (this is actually the geometrically intersection), all data points were fitted into another form of Meyer's law in Equation(2-28). Yield strength is then calculated using an empirical Equation (2-29). Unfortunately, different $\beta_{m}$ applies for different material.

$$
\begin{aligned}
& d_{t}=2 \sqrt{h_{t} D-h_{t}^{2}} \\
& \frac{P}{d_{t}^{2}}=A\left(\frac{d_{t}}{D}\right)^{m-2} \\
& \sigma_{y}=\beta_{m} A
\end{aligned}
$$

Following Tabor, Lubos et $\mathrm{al}^{[42]}$ showed that a constraint factor 2.45 works better for plasma sprayed nickel-aluminum coating. More discussion will be given in the indentation-induced deformation analysis. 


$$
\sigma_{\text {flow }}=\frac{P_{\text {contact pressure }}}{2.45}
$$

It should be noted that for geometrically similar indenter, only one point on the flow curve can be obtained. Lenhart ${ }^{[2]}$ also showed for some material such as magnesium which has high anisotropy of deformation, Tabor's relation is not exactly true.

\section{Indentation-induced deformation}

The indentation-induced deformation includes a permanent indent and the surrounding area deformation, such as piling-up or sinking-in. Shallowing effects were observed after the indentation test, which means after the load is released, the residual indent has a larger included angle than that of the indenter surface. Foss and Brumfield (1922) ${ }^{[1]}$ showed the indentation is still of a spherical form for any spherical indenters. The piling-up and sinking-in effects will contribute some uncertainty to the diameter of the indentation.

Residual deformation/profile near the indentation area has been studied long time ago. Cracks, slip lines, sink-in and pile-up were observed for different materials. Different materials show different deformation modes after a cylindrical punch using a "Schlieren" optical method $(1930 \mathrm{~s})^{[12]}$.

Underwood (1970s) ${ }^{[22]}$ is probably the first one to use optical interferometry (each fringe represents $\sim 0.254 \mu \mathrm{m}$ out-of-plane deformation) to study the indentation-induced residual deformation. It was noticed that the presence of residual stress in the specimen results in un-symmetric fringe pattern in the indentation-induced residual out-f-plane deformation fields. By studying the change of fringe order of out-of-plane deformation, Underwood concluded that it is possible to quantitatively determine the residual stress (before indentation tests). He also pointed out that the irregularity of the fringe pattern near the contact region was due to the Lüder's strain effects.

Residual profile/out-of-plane deformation has also been correlated to Lüder's strain by Haggag $^{[4]}$. It showed that for increasing Lüder's strain exhibited by the material, the number of interference fringes around the corresponding indentation increases.

Both in-plane and out-of-plane residual deformation after Vickers indentation (294N to $2435 N$ ) were studied by M. Szutkowska (1999) ${ }^{[45]}$. The residual fields were accomplished using high sensitivity moiré interferometry and Twyman-Green interferometry, but no further analysis has been reported. For brittle material, fracture toughness $\mathrm{K}_{\mathrm{IC}(\mathrm{HV})}$ requires the measurement of the length of the radial cracks formed at the end of the diagonals of the Vickers indent at loads which exceeds the critical load $\mathrm{P}_{\mathrm{C}}$. For the radial cracks under the condition $0.25 \leq l / a \leq 2.5$ the data can be described by Equation (2-31). Bur fracture toughness measurement has not been standardized because first, the appearance of cracks is difficult to 
observe, and secondly, the critical load and the crack length at loads higher than the critical load are extremely sensitive to stresses introduced during the preparation of the test surface by grinding and polishing ${ }^{[45]}$.

$$
\left(K_{I C} \phi / H \sqrt{a}\right)(H / E \phi)^{2 / 5}=0.035(l / a)^{-1 / 2}
$$

Where $\phi$ is the constraint factor $(\approx 3), H$ is the hardness, $E$ is the Young's modulus, $a$ is half-diagonal length of the Vickers indenter, $l$ is the radial crack length as shown in Figure $2.7^{[45]}$.

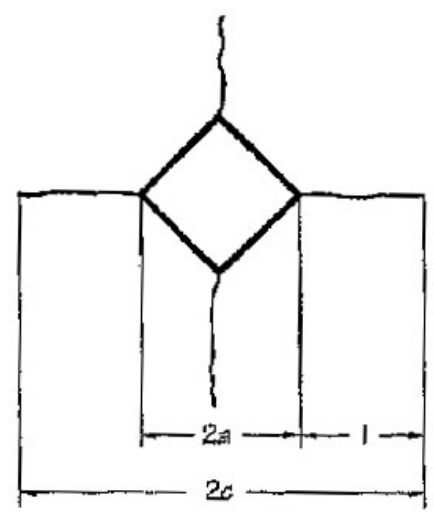

Figure 2.7 Illustration of radial cracks geometry of Vickers indentation

(M. Szutkowska ${ }^{[45]}$ )

Alcala et $\mathrm{al}^{[6]}$ showed that the surface residual profile is closely related to material strain hardening coefficient. Based on series indentation tests and corresponding residual surface profiles studies using profilometer, it is concluded that sinking-in predominates in material where $n>0.2$. The contact radius or contact area is calculated using the contact depth, which is determined according to the strain hardening coefficient using the relationship described by Equation (2-32). This study leads to a better estimation of key indentation parameter, contact area/contact radius from the load-depth data, thus improves the assessment of Young's modulus, hardening exponent and yield strength.

$$
h=h_{s} c^{2}=\frac{a^{2}}{D}
$$

And $c^{2}-1=0.276-1.748 n+2.451 n^{2}-1.469 n^{3}$

Where $h$ is the contact depth, $h_{s}$ is maximum depth of penetration underneath the original surface, $c^{2}-1$ sets the transition from piling-up to sinking-in ( $>0$, piling up happens, $<0$, sinking-in happens).

Indentation residual internal deformation or sub-surface deformation has also been studied mainly through two approaches, namely, sectioning or bonded-interface. Both methods need delicate specimen preparation. For sectioning method, after indentation, the specimen is cut along the symmetric axis of the indent. Then on the fresh surface, usually microindentation is 
used to indirectly determine the equivalent plastic strain ${ }^{[42]}$. While bonded-interface method cuts the specimen first and makes marks on the section, then bond them together to be an intact specimen. The indentation on the bonded specimen needs delicate alignment with the indenter.

Using bonded-interface method, Lubos et al (2003) ${ }^{[42]}$ studied the subsurface deformation for nickel-5\%aluminum plasma sprayed coating. Based on the stress-strain relation obtained using Tabor's empirical relation ( $C=2.45, E$ is from unloading stiffness measurement), finite element simulation was conducted to cross verify the sub-surface deformation and stress-strain distribution. The sub-surface deformation was quantified by measuring the deformation of the Vickers indentation marks (compared with the image taken before indentation).

\section{Load-depth sensing indentation}

Instrumented indentations have made it possible that force and depth are recorded continuously as an indentation is made. Figure 2.8 shows the typical indentation process kinetic diagram, the so called P-h curve. Quantitative analysis of this diagram is required in order to correlate the P-h curve to the material mechanical properties. Analytical solutions are difficult to obtain for general indentation applications. Much of the understanding of the indentation process has been acquired through experiments and finite element simulations. But for pure elastic and elastic-perfect plastic case, theoretical solutions may be obtainable.

The most direct results from P-h curve are Young's modulus. The contact radius/contact area is the most critical parameter and is frequently estimated from the P-h curve. So Young's modulus can be derived from initial unloading stiffness. If multiple loading-unloading is conducted, post-yielding stress-strain data can also be derived the P-h curve.

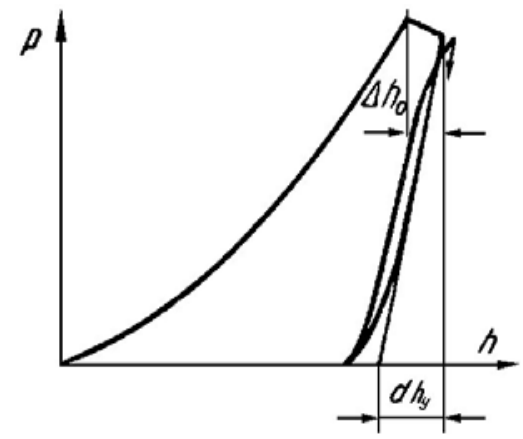

Figure 2.8 Typical indentation load-depth relation (Bulychev ${ }^{[28]}$ )

The earliest load-depth sensing indentation experiments were performed by Tabor ${ }^{[1]}$. Based on the series indentation tests on metals, it was experimentally verified that indentation unloading is an elastic process, the so called elastic recovery. The result leads to the elastic contact analysis of indentation unloading. The practical classical elastic indentation solution should owe to Sneddon (1965) $)^{[16]}$ in which he derived general relationships among the load, displacement and contact area for any punch that can be described as a solid of revolution of a smooth function while Boussinesq's elastic solution has practical computational difficulty. 
For a flat-ended cylindrical punch, explicit relations among load, indentation depth, stress distribution and deformation distribution have been given by Sneddon in Equation (2-34) to (2-36).

$$
\begin{aligned}
& P=\frac{4 G a h}{1-v}=\frac{2 E a h}{1-v^{2}} \\
& \sigma_{z z}(\rho, 0)=-\frac{2 G h}{\pi(1-v)}\left(a^{2}-\rho^{2}\right)^{-\frac{1}{2}}, 0 \leq \rho \leq a \\
& u_{z}(\rho, 0)=\frac{2 h}{\pi} \sin ^{-1}(a / \rho), \rho>a
\end{aligned}
$$

Where $G=\frac{E}{2(1+v)}, h$ is the depth of penetration, $v$ is Poisson's ration

The results for a conical punch is,

$$
\begin{aligned}
& P=\frac{\pi G a^{2}}{1-v} \cot \alpha=\frac{\pi E a^{2}}{2\left(1-v^{2}\right)} \cot \alpha, \text { or } \\
& P=\frac{4 G \tan \alpha}{\pi(1-v)} h^{2}=\frac{2 E \tan \alpha}{\pi\left(1-v^{2}\right)} h^{2} \\
& \sigma_{z z}(\rho, 0)=-\frac{2 G h}{\pi a(1-v)} \cosh ^{-1}(a / \rho), 0 \leq \rho<a \\
& u_{z}(\rho, 0)=\frac{2 h}{\pi a}\left\{\operatorname{asin}^{-1}(a / \rho)-\rho+\sqrt{\left(\rho^{2}-a^{2}\right)}\right\}, \quad \rho>a
\end{aligned}
$$

Where $\alpha$ is the semi-included apex angle, $a$ is the contact radius, and $h=\frac{1}{2} \pi \varepsilon=\frac{\pi}{2 \tan \alpha} a$

The spherical indenter is much more complex. Sneddon only showed the explicit relation for indentation load. Deformation and other detailed information are derived by Johnson ${ }^{[32]}$.

$$
\begin{aligned}
P & =\frac{G}{1-v}\left\{\left(a^{2}+R^{2}\right) \log \frac{R+a}{R-a}-a R\right\} \\
& =\frac{E}{2\left(1-v^{2}\right)}\left\{\left(a^{2}+R^{2}\right) \log \frac{R+a}{R-a}-a R\right\}
\end{aligned}
$$

and $h=\frac{1}{2} a \log \frac{R+a}{R-a}$

A different set of load-depth relation was obtained by Lure $^{[30]}$, 


$$
\begin{array}{ll}
P=\frac{d}{k_{0}} h & \text { for cylindrical/flat-end punch } \\
P=\frac{4}{3} \cdot \frac{\sqrt{R}}{k_{0}} h^{3 / 2} & \text { for spherical punch } \\
P=\frac{2}{\pi} \frac{1}{k_{0} \cot \varphi} h^{2} & \text { for conical (sharp tip) punch }
\end{array}
$$

where $k_{0}=\left(1-v^{2}\right) / E_{d}, v$ and $E_{\mathrm{d}}$ are Poisson's ratio and Young's modulus of the specimen, $h$ is the elastic indentation depth, $d$ is the diameter of the cylindrical punch or the diameter of the contact area, $R$ is the radius of the spherical punch, $2 \varphi$ is the included angle at the apex of the conical punch.

It should be noted that for conical punches, $h=\frac{\pi}{4} d \cdot \operatorname{ctg} \varphi$, and for spherical punch, $R=\frac{d^{3}}{6 P k_{0}}, h=\frac{3}{2} \frac{P k_{0}}{d}$ (Note: the contact happens at the half elastic depth).

In summary, the load-depth relationship for many simple punch geometries can conveniently be written as,

$$
P=\alpha h^{m}
$$

Where $P$ is the load, $h$ is the elastic displacement of the indenter (for elastic-plastic material, it refers to the recovered depth during unloading process, not the total depth), and $\alpha, m$ are constants. Values of $m$ are 1, 1.5 and 2 for flat cylindrical, spherical and conical punches respectively.

Considering the unloading as a reversible elastic process, Bulychev and co-workers $(1975)^{[28]}$ defined the initial unloading slope and reduced modulus, thus provided a theoretically sound methodology for determining the modulus of elasticity which is applicable to both spherical and pyramidal indenters. Their studies also showed that the linear dimension of the specimen should be at least ten times bigger than the cross-section dimensions of the indentation.

The initial unloading derivatives of Equation (2-41) to (2-43) lead to the same results,

$$
\frac{d P}{d h}=\frac{d}{k_{0}}
$$

Or in terms of reduced modulus,

$$
\frac{d P}{d h}=d E_{r}
$$


It can also be expressed in the terms of the projected area,

$$
\frac{d P}{d h}=\frac{2}{\sqrt{\pi}} \frac{\sqrt{\mathrm{A}}}{k_{0}} \quad \text { or } \quad \frac{d P}{d h}=\frac{2}{\sqrt{\pi}} E_{r} \sqrt{A}
$$

Realistic indenters are not rigid, if considering the elastic indenter and the interferometric between the indenter and holder, the reduced modulus is defined as,

$$
\frac{1}{E_{r}}=\frac{1-v^{2}}{E_{d}}+\frac{1-v^{2}}{E_{i}}
$$

Where $E_{i}$ is the indenter's Young's modulus.

Bulychev ${ }^{[28]}$ pointed out that for a pyramidal indenter, Equation (2-47) is also applicable with good accuracy. Further, Pharr, Oliver and Brotzen (1992) ${ }^{[31]}$ showed that Equation (2-47) can be applied to any indenter that can be described as a body of revolution of a smooth function. Through finite element simulation, King (1987) ${ }^{[18]}$ proved this formula also works well for a indenter which is not a body of revolution. For square and triangular cross sections the deviations from flat ended punches are only $1.2 \%$ and $3.4 \%$, respectively.

The system compliance was also carefully studied ${ }^{[28]}$ by considering the measurements shown in Figure 2.9,

$$
\begin{aligned}
& \frac{d h_{y}}{d P}=\frac{\sqrt{\pi}\left(k_{0}+k_{i}\right)}{2 \sqrt{A}}+\frac{d h_{h}}{d P}, \text { or } \\
& \frac{d h_{y}}{d P}=\frac{\sqrt{\pi}}{2 E_{r} \sqrt{A}}+\frac{d h_{h}}{d P}
\end{aligned}
$$

Where $\frac{d h_{h}}{d P}$ is the compliance of the interface between diamond and holder.

The indenter also indents the holder. Because the contacting area between holder and indenter is constant, $\frac{d h_{h}}{d P}$ can be set as constant and obtained through calibration.

For a small load, the deformation of the holder is negligible, Equation (2-49) is written as,

$$
\frac{d h_{y}}{d P}=\frac{\sqrt{\pi}\left(k_{0}+k_{i}\right)}{2 \sqrt{A}}+\frac{d h_{h}}{d P}=\frac{\sqrt{\pi}\left(k_{0}+k_{i}\right)}{2 \sqrt{A}}+\frac{\sqrt{\pi}\left(k_{h}+k_{i}\right)}{2 \sqrt{A_{h}}}
$$

Thus, the system compliance can be accurately calculated by assuming two indentation tests happened as shown in Figure 2.9.

For large loading, the deformation of the holder must be taken into account and the system calibration is necessary in order to find exactly $\frac{d h_{y}}{d P}-\frac{d h_{h}}{d P}=\frac{\sqrt{\pi}\left(k_{0}+k_{i}\right)}{2 \sqrt{A}}$. After all the calibrations, by assuming Poisson ratio equals 0.3 for unknown material (for $v$ lies $0.25 \sim 0.35$ ), the calculated Young's modulus $E_{d}$ is estimated to have $3 \%$ error $^{[28]}$. 


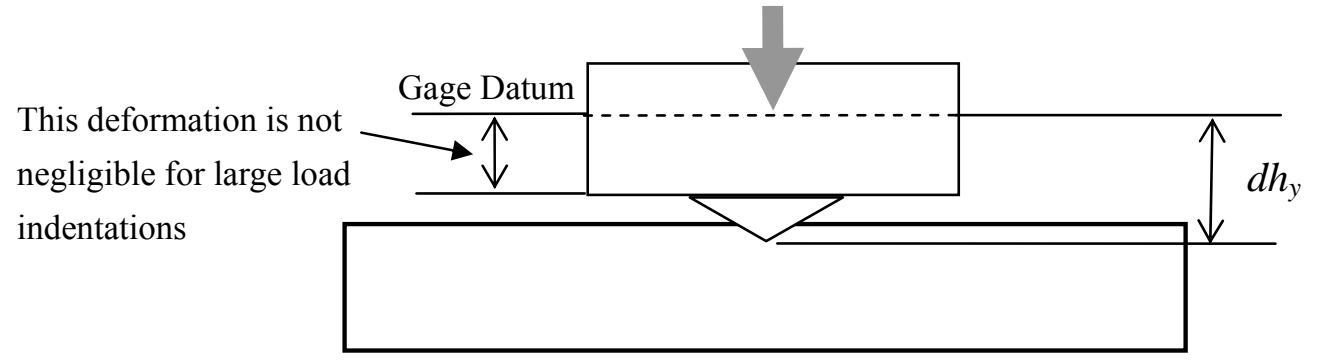

Figure 2.9 Penetration depth measurements

Vickers depth-sensing indentation on brittle material magnesium oxide was conducted by Loubet et al(1984) $)^{[17]}$. In their indentation setup, indentation depth is directly measured using displacement gage, thus the error from the rigidity of the frame is minimized. The initial unloading stiffness is used to calculate the Young's modulus. All the unloading curves are not straight lines, which are also pointed out by Bulychev et al earlier ${ }^{[28]}$. After Vickers indentation, the indents were inspected by SEM and optical microscope. The residual indentation depth, elastic rebound depth, grooves of plastic deformation, dislocations were also observed and analyzed. The extent of surface cracking around the corners of a Vickers pyramid indentation reflects the fracture toughness and the state of the surface stresses in a brittle material. But the comparison between different works introduced by the indentation test manifested that the work of fracture (Surface energy of $\mathrm{MgO}$ times crack surface area) is negligible compared with elastic and plastic work. The ratio of the residual depth and total penetration depth was found to be a constant $(0.69 \pm 0.002)$ for different loading and it may be a material constant.

Thin-film mechanical property evaluation using indentation technique requires very small load, thus conventional directly microscopic imaging method may introduce large error in the determination of the contact radius or contact area, especially when the indent is very small. It was then recognized that depth-sensing indentation can provide the necessary resolution and repeatability in the evaluation of thin-film properties. Doerner and Nix (1986) ${ }^{[46]}$ studied the thin-film mechanical properties using loading-unloading indentation through Berkovich Nanoindenter from Nano Instruments Inc. By introducing the total depth $h_{\text {total }}$, plastic depth $h_{\text {plastic }}$, final depth $h_{\text {final }}$, and the indenter shape function, both Young's modulus and hardness were obtained from the load-depth data using plastic depth (Figure 2.10). Equation (2-47) can be re-written by equating the projected area to the Vickers indenter project area, thus the initial slope of the P-h curve is written as,

$$
\frac{d P}{d h}=\left(\frac{2}{\pi}\right)^{1 / 2} D E_{r}
$$

If it is assumed that the indenter has an ideal pyramidal geometry and uses the plastic depth $h_{p}$ instead of the diagonal length, then, 


$$
\frac{d h}{d P}=\frac{1}{2 h_{p}}\left(\frac{\pi}{24.5}\right)^{1 / 2} \frac{1}{E_{r}}
$$

Since the Berkovich and Vickers indenter have the same area to depth relation, Equation (2-52) holds for either type of the indenter.

For nanoindentation, it is found that determination of the exact indenter shape is critical, so as the contact area, to the measurements of both hardness and Young's modulus. By calibrating the indenter using replica and TEM measurements, the effective depth is obtained,

$$
h_{\text {eff }}=\left(\frac{\text { Area }}{24.5}\right)^{1 / 2}
$$

Most important, for thin film applications, the influence of the substrate on the unloading stiffness can be modeled empirically using,

$$
\frac{d h}{d P}=\frac{1}{2 h_{p}}\left(\frac{\pi}{24.5}\right)^{1 / 2}\left(\frac{1-v_{f}^{2}}{E_{f}}\left(1-e^{-a t / h_{\text {eff }}}\right)+\frac{1-v_{s}^{2}}{E_{s}}\left(e^{-a t / h_{\text {eff }}}\right)+\frac{1-v_{0}^{2}}{E_{0}}\right)+b_{s}
$$

Unfortunately, they did not pay much attention to the depth-dependence of measured

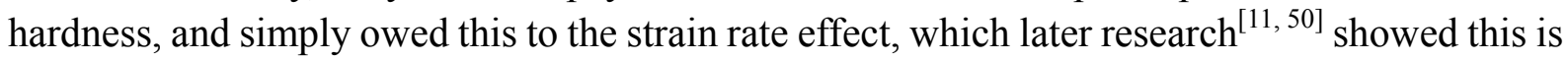
due to length scale effects.

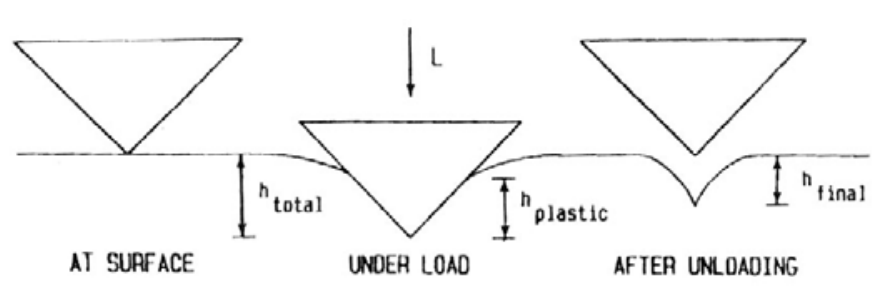

Indentation process

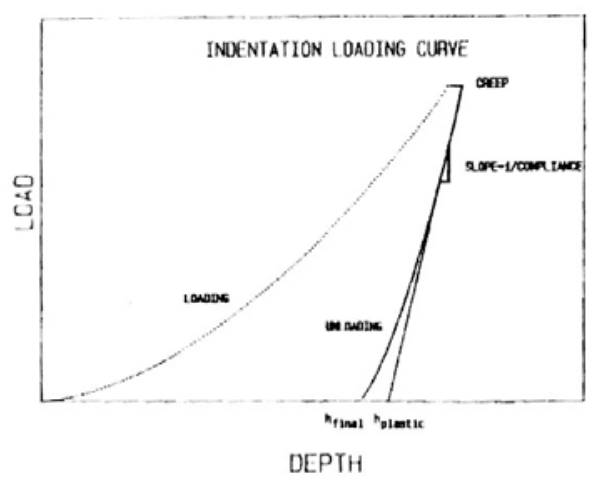

Load-depth curve showing definition of depth $\mathrm{h}$

Figure 2.10 Indentation depth definition

(Doerner et al ${ }^{[46]}$ )

Systematically experimental investigation of the unloading characteristics were also conducted by Oliver and Pharr (1992) ${ }^{[15]}$. Six different materials (aluminum, tungsten, soda-lime glass, fused silica, sapphire and quartz) were carefully studied using Nano-indenter ${ }^{\circledR}$ (Nano Instruments, Inc.). The continuous stiffness measurements confirmed the continuous change of the unloading stiffness. By rearranging the unloading data, so that all the unloading curves passed through a common origin, it was found they followed the same power law form as described in Equation (2-55). Further studies showed that $m$ is a material constant, which is always greater than 1.0 and varies from one material to another in the range of 1.25 to 1.51 .

$$
P=A\left(h-h_{f}\right)^{m}
$$


Where $h_{f}$ is the residual depth of the impression, $h-h_{f}$ can be viewed as elastic depth.

Table 2.3 Parameters describing power law fits of unloading curves (Oliver and Pharr ${ }^{[15]}$ )

\begin{tabular}{lccc}
\hline Material & $\mathrm{A}(\mathrm{mN} / \mathrm{nmm})$ & $\mathrm{M}$ & Correlation Coefficient \\
\hline Aluminum & 0.265 & 1.38 & 0.999938 \\
Quartz & 0.0215 & 1.43 & 0.999985 \\
Soda-lime glass & 0.0279 & 1.37 & 0.999997 \\
Sapphire & 0.0435 & 1.47 & 0.999998 \\
Fused silica & 0.05000 & 1.25 & 0.999997 \\
Tungsten & 0.141 & 1.51 & 0.999986 \\
\hline \hline
\end{tabular}

For both Young's modulus and hardness measurement, the real contact depth (so as the contact area, or contact radius) is a key parameter. Based on Sneddon's solution, a new analytical procedure was proposed by Oliver and Pharr ${ }^{[15]}$ to calculate the real contact depth,

$$
\begin{aligned}
& h_{c}=h_{\max }-h_{s} \\
& h_{s}=\varepsilon \frac{P_{\max }}{S}
\end{aligned}
$$

Where $\varepsilon$ is an indenter dependent parameter.

$\varepsilon=\frac{2}{\pi}(\pi-2)=0.72$ for conical indenter,

$\varepsilon=1.0$ for flat punch,

and $\varepsilon=0.75$ for paraboloid of revolution.

Then the real contact area at maximum loading status is calculated as,

$$
A\left(h_{c}\right)=24.5 h_{c}^{2}
$$
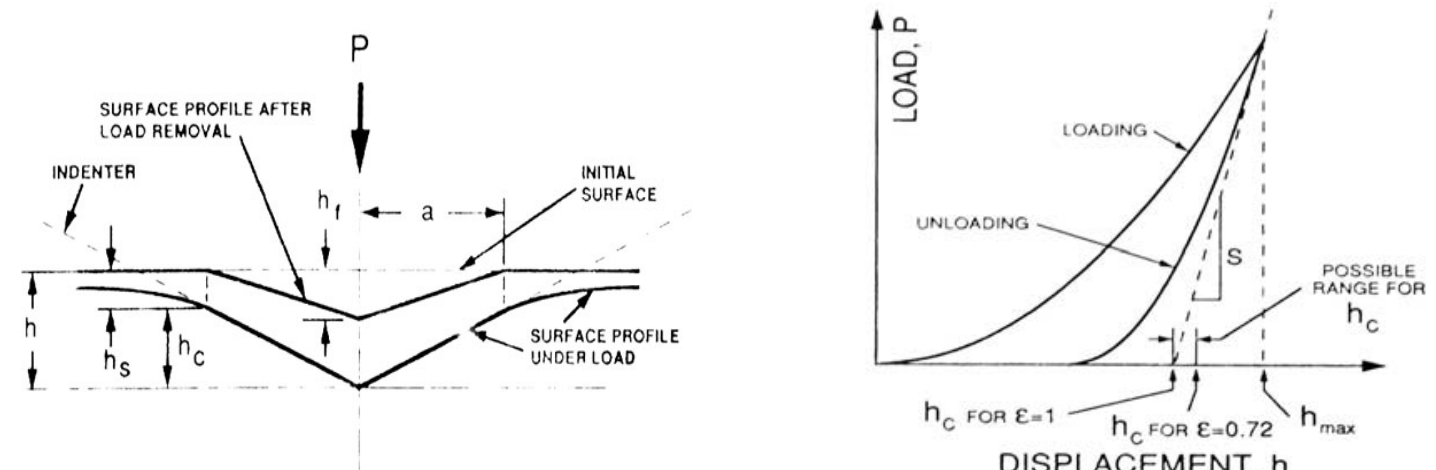

Figure 2.11 Definition of various quantities used in indentation analyses (Oliver and Pharr ${ }^{[15]}$ )

For geometrically similar indenter, such as cone or pyramid indenters, stress-strain 
relationship cannot be uniquely determined from the load-unloading curve. Yang-Tse Cheng et $\mathrm{al}^{[47]}$ have showed that different stress-strain curve can result the same loading-unloading curve.

\subsection{Thin film indentation analysis}

The thin-film (coating) application is one of the main reasons that depth-sensing indentation technique is of great interest recently. The indentation analyses of the thin-film material are similar to that of bulk materials except that the materials become more complex since more than one material is involved in the specimen. Part of the information has been reviewed in §2.3, and more finite element analyses will be discussed in §2.5.

\subsection{Finite element simulation of indentation}

Experimental work is difficult and time-consuming with limited measurement capabilities. Instead, fully elastic-plastic finite element analysis (FEA) provides a powerful tool to study the indentation process. Through FEA, all the parameters can be analyzed to correlate to material mechanical properties for both bulk and thin-film materials. Since the indentation process usually involves large deformation, both geometric nonlinearity and material nonlinearity need to be considered. Software-wise, most of the FEA package, such as ABAQUSTM ${ }^{\mathrm{T}}$ ANSYS ${ }^{\mathrm{TM}}$, can be used for simulation purpose.

From the simplest to the most complicated case, 1D model, 2D model or 3D model may be used to analyze the indentation process depending on different applications. (a) 1D model: Model indentation as concentrated force. This is the simplest model, and can be used to study the material behavior away from the indentation impression ${ }^{[23]}$. (b) 2D model: Model indentation in 2D space. This is generally applicable to axisymmetric conditions which greatly reduce the complexity and computational time. Most of the detailed indentation analyses fall into this category ${ }^{[26-28,36-40,42]}$. (c) 3D model: Model indentation in 3D space. This is the most complicated model and can generally be applied to any indentation tests. Quarter, half or full model can be used. For symmetric case, most computational economic one is the $1 / 4$ model. $1 / 2$ and full model size are used for different purposes ${ }^{[24-25,41]}$.

\section{D Modeling}

1D modeling is seldom used and it is only suitable for far field study. It has been reported in the strain-gage-based indentation residual stress measurement by Lin et $\mathrm{al}^{[23]}$. The indentation applied to the specimen is simplified as a concentrated force, but the specimen can be modeled in any space. This 1D modelling avoids complicated contact problem in the simulation. This simplification is based on the facts that the indenter used in such measurements is small $(\leq 2 \mathrm{~mm})$ and strain gage is located about $2.5 \sim 5.0 \mathrm{~mm}$ from the center of the impression. According to St. Venant theory, the indentation effect should be localized and 
such simplification should not affect the results. It has been showed that the axial strain change has linear relationship with the axial stress around the indentation under certain experimental conditions.

Different from other papers; quadratic relation between $\Delta z$ (concentrated force down movement) and load $P$ is obtained from $1 D$ model indentation simulation ${ }^{[23]}$. Residual stress presented before the indentation will affect the curve parameters. At a fixed location, the strain change has linear relationship with residual stress and this relation can be obtained through pre-calibration.

\section{D Modeling}

2D modeling of the indentation process is useful for most of the indentation simulations. From the simulations, all indentation parameters can be analyzed to correlate to material mechanical properties, such as Young's modulus, hardness, yield strength and post-yielding stress-strain data.

Bhattacharya and Nix (1988) $)^{[26-27]}$ studied indentation experiments for both bulk and thin-film materials (ABAQUS ${ }^{T M}$ ). For the elastic-plastic isotropic bulk material, it has been demonstrated that Young's modulus can be ascertained successfully from the linear portion of the unloading curve, and the hardness was more or less independent of the depth of indenter under simple material constitutive equations.

In their study for thin-film materials, the elastic and plastic properties of both the film and substrate on the hardness of the film-substrate composite were studied by determining the average pressure under the indenter as a function of the indentation depth. It's found that the hardness increases with indentation depth for either the yield strength or the elastic modulus of the substrate is bigger than that of the film. Correspondingly, the hardness decreases with indentation depth when the yield strength or elastic modulus of the substrate is smaller than that of the film. Also, from the FEA, the elastic compliance of the film-substrate composite as a function of indentation depth was developed. Using this function, the elastic properties of the film can be separated from those of the substrate. It's also shown that friction between the indenter and the specimen has no noticeable effect on the load-depth curve under perfect bonding condition. Based on the flat-end punch model, the difference of Young's modulus using different depth was studied and projected area was the best compared with all other depth solutions. The yield stress and Young's modulus effects on the load-depth curve was also systematically studied and general relations for the hardness variation was obtained. These equations are basically non-dimensionalized and thus can be used for any film thickness involving any material combination of the film and substrate. Equation (2-59) is used to calculate the Young's modulus. Equations (2-60) to (2-62) are used to describe the thin-film materials.

$$
E=\frac{1-v^{2}}{2 h_{p}} \sqrt{\left(\frac{\pi}{24.5}\right)} \frac{d p}{d h}
$$

For soft film on a harder substrate, 


$$
\frac{H}{H_{s}}=1+\left(\frac{H_{f}}{H_{s}}-1\right) \exp \left[-\frac{\sigma_{f} / \sigma_{s}}{E_{f} / E_{s}}\left\{\frac{t}{t_{f}}\right\}^{2}\right]
$$

For hard film on a softer substrate,

$$
\frac{H}{H_{s}}=1+\left(\frac{H_{f}}{H_{s}}-1\right) \exp \left[-\frac{H_{f} / H_{s}}{\sigma_{f} / \sigma_{s} \sqrt{E_{f} / E_{s}}}\left\{\frac{t}{t_{f}}\right\}\right]
$$

But for thin-film material, the compliance used for Young's modulus calculation is indentation depth dependent,

$$
\frac{d h}{d P}=\frac{1}{\beta \sqrt{A}}\left[\frac{1-v_{f}^{2}}{E_{f}}\left\{1-\exp \left(-\frac{\alpha^{\prime} t_{f}}{\sqrt{A}}\right)\right\}+\frac{1-v_{s}^{2}}{E_{s}}\left\{1-\exp \left(-\frac{\alpha^{\prime} t_{f}}{\sqrt{A}}\right)\right\}\right]
$$

Detailed stress-strain and deformation analyses have been conducted both in elastic and elastic-plastic cases. Matthewson, Ihara, Barovich, $\mathrm{Ku}$, van der Zwaag and Field et al ${ }^{[36]}$ studied the elastic case stress field for different thin films. Komvopoulos and co-workers $(1988)^{[36-41]}$ systemically studied the cylindrical indentation (plane-strain case) on layered medium using elastic and elastic-plastic material model. From the elastic simulations, the mechanism of micro-crack initiation at the layer surface or interface, layer debonding, and onset of plastic flow in the layered solid were explained qualitatively in the light of governing stresses and the regimes of their prevalence were approximately determined. It has been shown that the layer thickness and the mechanical properties of the layer and the substrate significantly affect the contact pressure. A sufficiently thick and hard layer will be especially advantageous in reducing plastic deformation for the substrates. Yielding in the layered medium always initiates at the layer-substrate interface below the center of the contact. Converse to the non-layered medium, the plastic zone does not grow toward the surface of the indented layered medium. Their investigation of 3D model will be discussed shortly.

But in their study, the repeated indentation may not be necessary since no deformation history has been considered in their simulation, and the final deformation only depends on the loading status.

The engineering size indentation has also been studied by Lubos ${ }^{[42]}$ (see $\$ 2.3$ for more detail) through 2D modeling. It showed that modified Tabor's relation is successfully used for nickel-5\%aluminum plasma sprayed coating/layered porous materials.

\section{D Modeling}

3D modeling is the most complicated case. It basically can be used for any indentation simulations, but it is more computational expensive. Depending on the application, either a quarter or half model can be used to reduce computational time. Also, for pre-present residual stress and sliding/scratch simulation, only a full 3D model may be applicable.

A half $3 \mathrm{D}$ indentation FEA model has been used by Kral et al ${ }^{[41]}$ to study the indentation 
and contact sliding effects under elastic-plastic material model. The surface characteristics resulting from indentation and sliding contact on a layered half-space were examined. The loading, sliding, unloading were all examined by considering different friction coefficient and normalized Young's modulus ratio ${ }^{[38-40]}$. The stress and displacements on the surface were used to study the failure mode and trends. The repeated load cycles were also applied to study the indentation characteristics.

A full 3D model may be required to study the pre-present residual stress. Because of the presence of residual stress, the model may not always deform axisymmetrically. Thus it is necessary to model the indentation process in the 3D space. It has been shown that for indentation residual stress measurement, strain gage should apply in the elastic zone and as near to the plastic zone as possible ${ }^{[23]}$. The strain increment is too small to be detected if the strain gage is far from plastic zone. It is experimentally found that for the same impression size, the strain increment in the elastic zone is proportional to the residual stress.

A quarter indentation FEA model has been used by Chen, $\mathrm{Yu}$ et $\mathrm{al}^{[24-25]}$ to study the residual stress effects on the elastic-plastic zone. Their study provides some basic understanding of experimental residual stress measurements. In their study, it is assumed work hardening has small influence on the plastic zone size, and elastic-perfect plastic material model was used. Both the indenter and specimen were modeled using 10 nodes 3D elements. The plastic zone size for impression of $1.0,1.1$ and $1.2 \mathrm{~mm}$ was systematically studied with different residual stress applied. It is found when impression reaches to $1.2 \mathrm{~mm}$, the maximum plastic zone size could be as large as $4 \mathrm{~mm}$. Under tension residual stress, the plastic zone size in tension direction does not change much and changes apparently in perpendicular direction. Under compression, the plastic zone size does not change much in compression direction but changes apparently in perpendicular direction. 


\section{PART 2 OPTICAL MEASUREMENT TECHNIQUE}

Optical methods play an important role in experimental stress analyses. Different from the strain gage measurement, optical methods usually yield full field measurements, which help to better understand how the material behaves. There is a broad selection of optical methods which are suitable for experimental stress analyses. Simple line mark, grid method and geometric moiré are easy to apply in which no coherent light source is needed. The digital image correlation (DIC) was developed from laser speckle phenomenon, but white light speckle digital image correlation is often used in engineering applications ${ }^{[55,58]}$. Even though white light is applicable for moiré interferometry and Michelson interferometer ${ }^{[57]}$, coherent laser light is still preferred in the modern interferometric measurements such as holographic interferometry, moiré interferometry, Twyman-Green interferometry, and electrical speckle pattern interferometry (ESPI) ${ }^{[53-55]}$. Some commercial products are available for engineering applications $^{[77-78]}$, and some are still better conducted in laboratory scale. In this dissertation, moiré and Twyman-Green interferometry are used and more details will be discussed.

The results of optical methods are most likely yield in the form of fringe patterns. In the old days, film cameras were used and most of the measurements were done on the photos with ruler or optical microscope. With the fast development of PC-based data/image acquisition system and CCD/CMOS camera, most of work is now accomplished using the computerized control and data/image acquisition system, which makes the digital image processing more important than ever. Displacement fields are the most common results from optical interferometry even though strain or stress fields may be obtained from the fringe mismatch or photoelasticity ${ }^{[53-55]}$. The displacement fields usually represent equal displacement lines or contours. Due to the intrinsic characteristics of optical methods, most of the fringe patterns only represent relative displacement or strain; it may need experience or understanding of certain physical phenomenon to determine the fringe orders.

\subsection{Introduction to interferometry}

The coherent light is narrow band (very narrow frequency range) while non-coherent light is broad band. The most frequently used coherent light source is laser. Using wave theory, the laser light can be described as,

$$
O=A \cos (\omega t+\varphi)
$$

Where $\omega$ is the light frequency, $\omega t+\varphi$ is the phase.

A complex variable form is often used due to the simplicity of the derivations ${ }^{[60]}$,

$Z=A e^{i(\omega t+\varphi)}$

Thus the light intensity can be defined as,

$$
I=Z \cdot Z^{*}=A^{2}
$$


If two coherent lights (same frequency) $Z_{1}, Z_{2}$ have the same polarization direction or at least have the common component in certain polarization direction, they can interfere with each other and form an interferogram as,

$$
\begin{aligned}
& Z=Z_{1}+Z_{2}=A_{1} e^{i\left(\omega t+\varphi_{1}\right)}+A_{2} e^{i\left(\omega t+\varphi_{2}\right)} \\
& I=Z \cdot Z^{*}=A_{1}^{2}+A_{2}^{2}+A_{1} A_{2} \cos \left(\varphi_{1}-\varphi_{2}\right)=A_{1}^{2}+A_{2}^{2}+A_{1} A_{2} \cos (\Delta \varphi), \text { or } \\
& I=A+B \cos (\Delta \varphi)
\end{aligned}
$$

It should be noted that the intensity of the fringe patterns has sinusoidal distribution.

Usually, the fringe contrast is defined as,

$$
\lambda=\frac{I_{\max }-I_{\min }}{I_{\max }+I_{\min }}
$$

Where $I_{\max }=A+B$ and $I_{\min }=A-B$

The best fringe contrast $(\lambda=1)$ can be achieved when the intensity of the two interferential laser beams matches (i.e. $A_{1}=A_{2}$ ). For other cases, fringe contrast always between 0 and 1 .

In practice, how to convert these fringe patterns into meaningful physical variable is what we are interested. This is accomplished through relating $\Delta \varphi$ to interested physical variables. For example, displacement fields $(u, v, w)$ may be correlated to phase $\Delta \varphi$,

$$
\Delta \varphi=f(u(x, y, z), v(x, y, z), w(x, y, z))
$$

Traditional fringe analyses are based on line-thinning and fringe counting, in which only maximum $(\Delta \varphi=2 \mathrm{n} \pi, I=A+B$, white fringe) and minimum $(\Delta \varphi=(2 \mathrm{n}+1) \pi, I=A-B$, black fringe) of the fringe patterns were used. The intermediate values are discarded. With the development of phase-shifting technique, all the intermediate $\Delta \varphi$ values can be converted to interested physical variable for analyses. More important, this technique can also improve and increase the fringe pattern processing automation process. Currently, there are basically two main categories to introduce the phase shifting ${ }^{[53-57,59]}$ : mechanical adjustment and optical wavefront manipulation. The mechanical-adjustment-based phase-shifting is typically introduced through a linear stage, rotary stage or piezoelectric actuator (PZT). The phase shifting based on optical wavefront manipulation is typical introduced using inserted wedges, waveplates, liquid crystal or other optical retarders to manipulate wavefront.

\subsection{Phase extraction algorithm and unwrapping}

The algorithm of phase extraction is closely related to the phase-shifting method. To solve the phase $\Delta \varphi$ in Equation (2-67), both phase-stepping and Fourier transformation methods have been developed ${ }^{[3-57,59-60,67]}$. Fourier transformation methods may be found in other literature and optical engineering books. Here only the phase-stepping technique is 
interested and discussed. For both cases, digital image processing ${ }^{[76]}$ technique is of great help to extract information from the fringe patterns.

Three unknowns are presented in Equation (2-67), so at least three equations, i.e. three fringe patterns, are needed to solve $\Delta \varphi$. In practice, three and four fringe pattern images are frequently used to extract the phase. More than four fringe patterns can also be used and the redundant information is used to reduce the noise and system error ${ }^{[80]}$.

For three fringe pattern algorithm, a constant phase-step $2 \pi / 3$ is introduced between consecutive fringe patterns, i.e.,

$$
\begin{aligned}
& I_{1}=A+B \cos (\Delta \varphi) \\
& I_{2}=A+B \cos \left(\Delta \varphi+\frac{2 \pi}{3}\right) \\
& I_{3}=A+B \cos \left(\Delta \varphi+\frac{4 \pi}{3}\right)
\end{aligned}
$$

Solve the above equations,

$$
\Delta \varphi=\tan ^{-1}\left(\frac{\sqrt{3}\left(I_{1}-I_{3}\right)}{2 I_{2}-I_{1}-I_{3}}\right)
$$

The phase $\Delta \varphi$ is then properly extended to $[-\pi, \pi]$ according to the sign of numerator and denominator.

For the four image algorithm, constant phase-step can be either known or unknown. Using $\pi / 2$ as the phase-step between consecutive fringe patterns, four equations will form an over-determined system,

$$
\begin{aligned}
& I_{1}=A+B \cos (\Delta \varphi) \\
& I_{2}=A+B \cos \left(\Delta \varphi+\frac{\pi}{2}\right)=A-B \sin (\Delta \varphi) \\
& I_{3}=A+B \cos (\Delta \varphi+\pi)=A-B \cos (\Delta \varphi) \\
& I_{4}=A+B \cos \left(\Delta \varphi+\frac{3 \pi}{2}\right)=A+B \sin (\Delta \varphi)
\end{aligned}
$$

And the phase $\Delta \varphi$ can be solved,

$$
\Delta \varphi=\tan ^{-1}\left(\frac{I_{4}-I_{2}}{I_{3}-I_{1}}\right)
$$

The above algorithm is also called constant 4 step phase shifting method.

If an unknown constant phase-step $2 \delta$ is used between consecutive fringe patterns, the four equations have exactly four unknowns, 


$$
\begin{aligned}
& I_{1}=A+B \cos (\Delta \varphi-3 \delta) \\
& I_{2}=A+B \cos (\Delta \varphi-\delta) \\
& I_{3}=A+B \cos (\Delta \varphi+\delta) \\
& I_{4}=A+B \cos (\Delta \varphi+3 \delta)
\end{aligned}
$$

Solve the above four equations to obtain the phase $\Delta \varphi$,

$$
\Delta \varphi=\tan ^{-1} \frac{\sqrt{\left[\left(I_{1}-I_{4}\right)+\left(I_{2}-I_{3}\right)\right]\left[3\left(I_{2}-I_{3}\right)-\left(I_{1}-I_{4}\right)\right]}}{\left|\left(I_{2}+I_{3}\right)-\left(I_{1}+I_{4}\right)\right|}
$$

To facilitate phase unwrapping, $\Delta \varphi$ is then extended to the domain of $[-\pi, \pi]$,

$$
\Delta \varphi(x, y)= \begin{cases}\Delta \varphi(x, y) & \text { when }\left(I_{2}-I_{3}\right)>0,\left(I_{2}+I_{3}\right)-\left(I_{1}+I_{4}\right)>0 \\ \pi-\Delta \varphi(x, y) & \text { when }\left(I_{2}-I_{3}\right)>0,\left(I_{2}+I_{3}\right)-\left(I_{1}+I_{4}\right)<0 \\ -\pi+\Delta \varphi(x, y) & \text { when }\left(I_{2}-I_{3}\right)<0,\left(I_{2}+I_{3}\right)-\left(I_{1}+I_{4}\right)<0 \\ -\Delta \varphi(x, y) & \text { when }\left(I_{2}-I_{3}\right)<0,\left(I_{2}+I_{3}\right)-\left(I_{1}+I_{4}\right)>0\end{cases}
$$

The above is also called equal four-step phase shifting method.

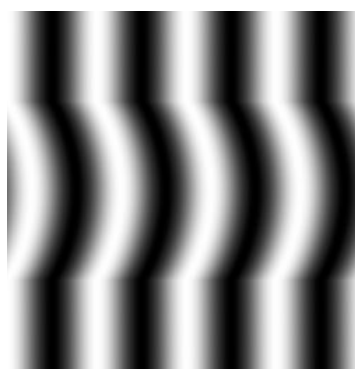

0

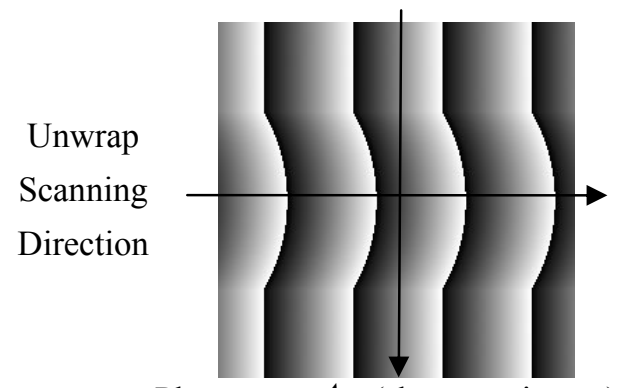

Phase map $\Delta \varphi$ (shown as image)

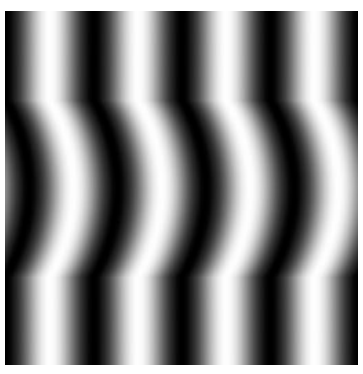

$\pi$

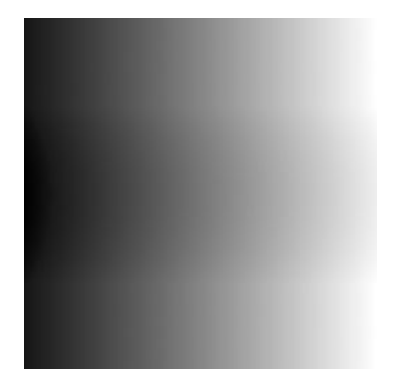

Unwrapped phase

Figure 2.12 Example of constant 4 step phase shifting and phase map

The phase $\Delta \varphi$ from most of the phase extraction methods is usually discontinuous as shown in Figure 2.12, in which the phase is quantified to a gray scale image, usually called phase map. The phase can only be extended to the domain of $[-\pi,+\pi]$ or $[0,+2 \pi]$ due to the trigonometric function. To restore the continuity of the original phase map, a proper phase unwrapping method has to be used. As shown in Figure 2.13, the principle of phase unwrapping and possible error pattern resulted from mis-corrected phase are illustrated using the classical line scan unwrapping method. Based on this simple principle, more unwrapping algorithms have been developed to cope with different applications and improve the unwrapping quality ${ }^{[61-66]}$. 
With noisy and irregular interesting area, unwrapping is not an easy task. Consistency checking has been proved to be able to identify some noisy locations, and regional unwrapping method is very useful for irregular unwrapping region.

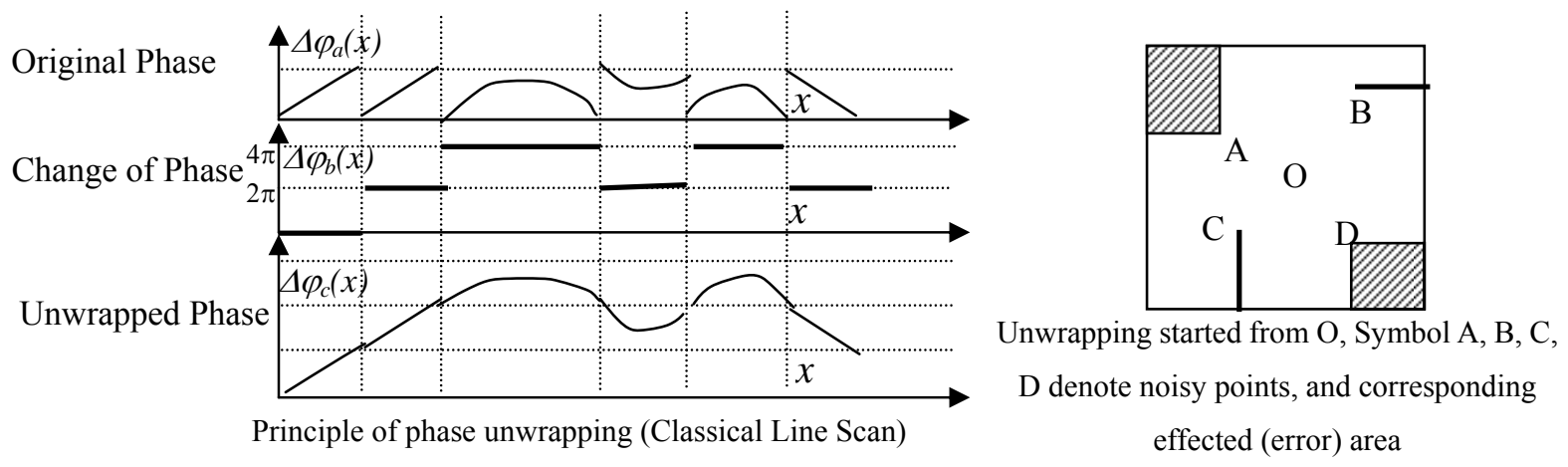

Figure 2.13 Unwrapping method and error patterns

\subsection{Twyman-Green interferometry}

The Michelson interferometry, using extended light source, is of historical significance in the development of Physics and related domain. It is still used in applications such as Laser Doppler interferometry ${ }^{[71]}$. It is also the basis of several interferometry-based measurement techniques, such as Twyman-Green interferometry, speckle interferometry and holographic interferometry. Basically, the Twyman-Green interferometry, patented in 1916, is derived from Michelson interferometry, in which F. Twyman and A. Green introduced lenses to create broad-field collimated light to Michelson interferometer.

The Twyman-Green configuration is frequently used for lens and mirror inspection due to its high sensitivity and high accuracy (half wavelength, $\lambda / 2$ ). In the study of material deformation, this is often used to measure surface out-of-plane deformation ( $w$ field).

The principle of Twyman-Green interferometry is shown in Figure 2.14. The phase $\Delta \varphi$ is related to Optical Path Difference (OPD),

$$
\Delta \varphi=\frac{2 \pi}{\lambda} \cdot O P D=\frac{2 \pi}{\lambda} \cdot 2 n \cdot(A B-B C)
$$

Where $n$ is the index of diffraction, $\mathrm{AB}$ and $\mathrm{BC}$ denote the length from beam splitter to the mirror or specimen.

The fringe pattern obtained from Twyman-Green interferometry reflects the relative change of the OPD. By correlating interested physical variable to OPD, the fringe pattern is used to explain the corresponding physical phenomena.

For white fringes, $\Delta \varphi=2 \pi \cdot m$, and black fringes, $\Delta \varphi=\pi \cdot(2 m+1)$. Difference between consecutive white (black) fringes is,

$$
\delta(\Delta \varphi)=2 \pi=\frac{2 \pi}{\lambda} \cdot \delta(O P D)=\frac{2 \pi}{\lambda} \cdot 2 n \cdot \delta(A B-B C)
$$


$\delta(A B-B C)=\frac{\lambda}{2 n}$

In air, $n \approx 1.0$, so $\delta(A B-B C) \approx \frac{\lambda}{2}$

Phase-shifting in Twyman-Green interferometry can be introduced by purposely changing the optical path using spatial carrier ${ }^{[69]}$ or PZT actuator ${ }^{[70]}$. Both can be used to further improve the measurement sensitivity and accuracy. It can also improve the measurement automation.

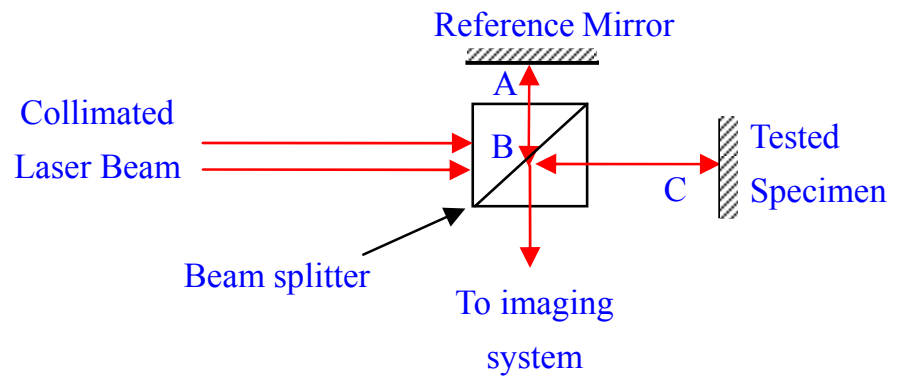

Figure 2.14 Principle of Twyman-Green interferometry

\subsection{Moiré interferometry}

Moiré interferometry is capable of high-sensitivity, whole field, in-plane displacement measurements $(u$ and/or $v$ field, each one is perpendicular to the corresponding grating direction). Compared with the electronic speckle pattern interferometry (ESPI), it offers a unique combination of high sensitivity, excellent contrast and spatial resolution. To conduct a moiré interferometry test, a high quality specimen surface and diffraction grating are required. Moiré interferometry needs much higher grating frequency than geometric moiré. Even through it can be explained in the same manner of geometrical moiré, the further understanding of moiré interferometry needs deep understanding of diffraction optics and optical path difference analysis.

The in-plane deformation fields can be used to evaluate the specimen surface deformation distribution and stress-strain distribution. Under small strain condition, it can be calculated as,

$$
\varepsilon_{x}=\frac{\partial u}{\partial x}, \quad \varepsilon_{y}=\frac{\partial v}{\partial y}, \quad \varepsilon_{x y}=\frac{1}{2}\left(\frac{\partial u}{\partial y}+\frac{\partial v}{\partial x}\right)
$$

\section{Diffraction grating}

There are two fundamental types of grating masters, ruled and holographic. Ruled gratings can be blazed for specific wave lengths and generally have higher efficiency. It can be produced on either flat or concave substrates. Holographic gratings often have lower scatter since they are generated optically, but they typically have excellent wavefront flatness and can have high efficiency in a single plane of polarization. Such gratings normally have a sinusoidal groove shape and they are frequently used in moiré interferometry. Diffraction 
gratings can also be categorized according to different aspect of the grating such as furrow \& cross-sectional lines, transmission $\&$ reflection and amplitude grating $\&$ phase grating.

As shown in Figure 2.15, positive angle is usually defined in the direction of counter-clock wise to the grating normal. For an incident beam with angle $\alpha$, a corresponding diffraction order $m$ with angle $\beta_{m}$ reflected from a diffraction grating with frequency $f$ (lines $/ \mathrm{mm}$ ), the diffraction equation can be written as ${ }^{[73-75]}$,

$$
\sin \alpha+\sin \beta_{m}=m f \lambda
$$

Where $m$ is the diffraction order.

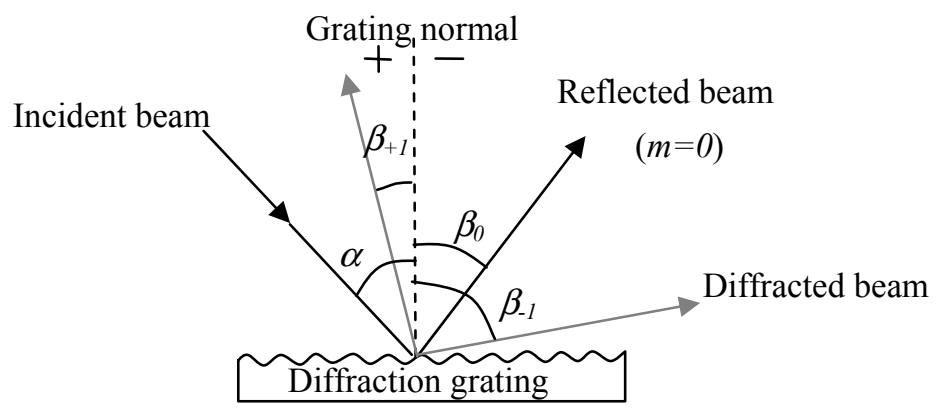

Figure 2.15 Diffraction grating

For moiré interferometry, specimen gratings are usually formed by a replication process. The grating is first produced on a glass plate by using two-beam interference. After developing, the non-uniform shrinkage of the gelatin-based photographic emulsion produces a wave pattern. To avoid destroying the expensive master grating, a sub-master grating made of silicone rubber is often used which is made from the original master grating. By using a master or sub-master grating mold, the metallic film coated on the surface of the master or sub-master grating can be transferred to the specimen through adhesive. If using ion-etching or chemical etching, zero thickness and high temperature grating can also be made on the specimen surface ${ }^{[53-55]}$.

\section{Principle of moiré interferometry}

Virtual grating ${ }^{[53-55]}$ is formed in the space where two laser beams meet with each other. Using the concept of virtual grating, moiré interferometry can be easily explained in terms of geometrical moiré.

For a traditional moiré interferometry setup, two incident laser beams are introduced symmetrically in the angle of $+\alpha$ and $-\alpha$. These two beams produce virtual grating in the overlap space in the frequency of,

$f_{v}=\frac{2 \sin \alpha}{\lambda}$

But from the diffraction Equation (2-81), the first order diffraction is written as, 
$\sin \alpha+\sin 0=f_{s} \lambda$

So, $\sin \alpha=f_{s} \lambda$ or $f_{s}=\frac{\sin \alpha}{\lambda}=\frac{f}{2}$

Where $f_{\mathrm{s}}$ is the specimen grating frequency.

After Comparing Equation (2-82) and (2-83), it is found that the frequency of the virtual grating is twice of the specimen grating.

As the specimen deforms, the grating moves and deforms along with the specimen surface. The specimen grating and virtual reference gratings interact to form a moiré fringe pattern, which is then viewed and photographed in the camera. This is similar to the geometric moire with reference grating of frequency $f_{v}$, and the sensitivity is controlled by the reference grating.

More rigorous explanation of the moiré interferometry is achieved through an optical path difference (OPD) analysis ${ }^{[54]}$. For the traditional moiré interferometry, the displacement-induced change of OPD for the diffraction order +1 and -1 can be described using Equations (2-84) and (2-85).

$$
\begin{aligned}
& \triangle O P D_{+1}=w(1+\cos \alpha)+u \sin \alpha \\
& \triangle O P D_{-1}=w(1+\cos \alpha)-u \sin \alpha
\end{aligned}
$$

The effective/total change of optical path difference is,

$$
\begin{aligned}
& \triangle O P D=\triangle O P D_{+1}-\triangle O P D_{-1}=2 u \sin \alpha \\
& \text { so } \triangle \varphi=\frac{2 \pi}{\lambda} 2 u \sin \alpha
\end{aligned}
$$

Using Equation (2-67), the fringe patterns are written as,

$$
I=A+B \cos \left(\frac{2 \pi}{\lambda} 2 u \sin \alpha\right)
$$

In terms of fringe orders $N_{x}$,

$$
\frac{2 \pi}{\lambda} 2 u \sin \alpha=2 \pi N_{x}
$$

So $u=\frac{N_{x}}{f_{v}}$

Where $f_{v}=\frac{2 \sin \alpha}{\lambda}$ 


\section{Development of moiré interferometry}

The traditional moiré interferometry offers a high quality full field in-plane deformation fringe pattern. Later development includes $w$ field assessment from moiré interferometry and further improvement of in-plane deformation measurement. This has been achieved either through introducing phase-shifting or increasing the measurement sensitivity.

For traditional moiré interferometry setup, if double exposure is applied with properly filtering, fields described by Equation (2-84) and (2-85) can be obtained separately. Addition of these two separately obtained interferential patterns leads to measurement of $w$ field ${ }^{[79]}$,

$$
\triangle O P D_{\text {add }}=\Delta O P D_{+1}+\Delta O P D_{-1}=2 w(1+\cos \alpha)
$$

Due to its high sensitivity and high spatial resolution, moiré interferometry has been used to measure small area deformation, such as crack tip and MEMS. High magnification moiré interferometer has been used for crack tip analyses by La Porta et $\mathrm{al}^{[80]}$. By applying fiber optics, the portable moire interferometer is very compact and is able to investigate the deformation of sub-millimeter area. This system is further improved by introducing phase-shifting capability through a combination of mechanical adjustment (PZT) and optical retarder (wedge) ${ }^{[80]}$.

The sensitivity of moiré interferometry has been doubled by a new optical configuration proposed by $\operatorname{Lin}^{[81]}$. In the new configuration, the incident laser beam is perpendicular to the specimen, and +1 and -1 diffraction order of the incident beam is reflected back to the original incident direction using two mirrors. The reflected beams of +1 and -1 diffraction orders are merged and then form the moiré fringe patterns. This configuration is easy to setup and adjust, and fewer optical components are used. Another advantage of this setup is that the laser wavefront directly from the laser is not critical to the testing results. But the quality of the fringe pattern is worse than traditional moiré interferometry because of the zero order effects. This configuration is not suitable for large deformation (such as crack-tip) application in which the diffraction is so severe that the mirror and the specimen grating cannot route all the light back to original incident beam direction.

The wave-manipulation-based phase-shifting moiré interferometry has been developed at West Virginia University under Dr. Kang's research group ${ }^{[82-84]}$. Creep crack growth and crack-tip deformation at both room temperature and high temperature were studied through zero thickness grating and/or high temperature moiré grating. By using liquid crystal variable retarder, phase-shifting is introduced other than mechanical adjustment. Figure 2.16 and Figure 2.17 show a schematic diagram of the optics layout and configuration. In this setup, a linear polarizer must be used in order to view/capture the image through CCD cameras. 


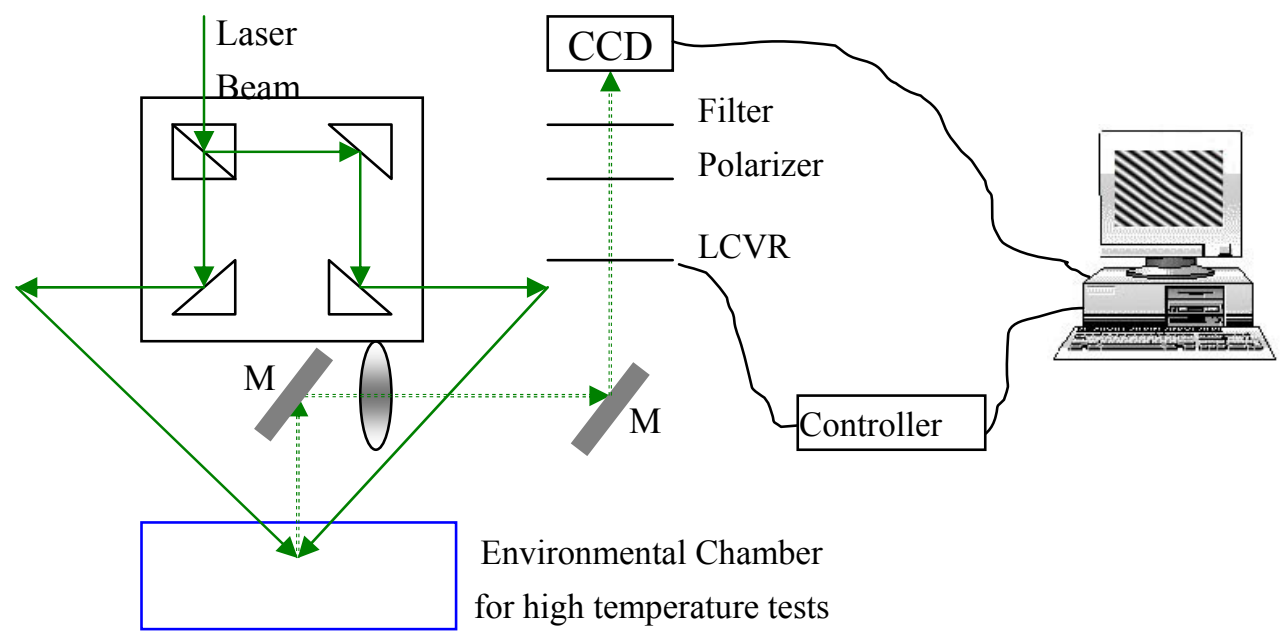

Figure 2.16 WVU Moiré interferometry with automatic phase shifting control

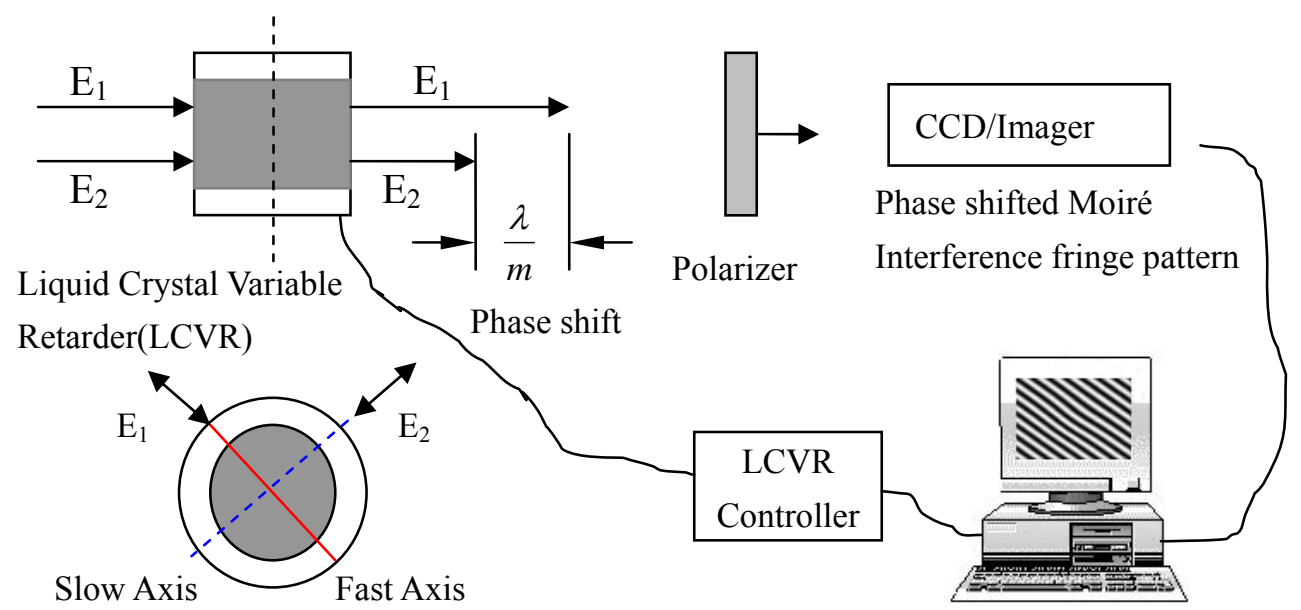

Figure 2.17 Optical phase shifting implementation by LCVR at WVU 


\section{CHAPTER 3}

\section{FINITE ELEMENT ANALYSIS}

Fully elastic-plastic finite element analysis (FEA) provides a powerful tool to study the indentation process. Through FEA, all the parameters can be analyzed to correlate to material mechanical properties for both bulk and thin-film materials. Since an indentation process usually involves large deformation, both the geometry nonlinearity and material nonlinearity are considered in the simulation. Software-wise, most of the FEA package, such as ABAQUS $^{\mathrm{TM}}$, ANSYS ${ }^{\mathrm{TM}}$, can be used. In this research, ABAQUS ${ }^{\mathrm{TM}}$ is chosen to accomplish the indentation simulation. With decades of development by HKS (Hibbitt, Karlsson \& Sorensen, Inc.), ABAQUSTM becomes one of the best powerful finite element simulation programs. It includes a whole family of products from pre-processing to final data analysis and visualization. It can solve problems ranging from relatively simple linear analysis to the most challenging nonlinear simulations.

Since the indentations on homogenous bulk material are mainly investigated in this research, a 2D axisymmetric finite element model is sufficient to study related indentation parameters. During the indentation process, the material underneath the indenter may undergo strain larger than 50 percent. Both the material nonlinearity (plasticity) and contact mechanics are involved in this problem. To solve this problem, ABAQUS ${ }^{\mathrm{TM}}$ uses the Newton-Raphson method to obtain the solutions. Based on the incremental theory of plasticity, ABAQUS ${ }^{\mathrm{TM}}$ breaks the simulation into a number of load increments and finds the approximate equilibrium configuration at the end of each load increment. It often takes several iterations to determine an acceptable solution to a given load increment. The sum of all the incremental response is the approximate solution for corresponding nonlinear analysis ${ }^{[14]}$.

\subsection{Introduction to indentation analysis in ABAQUS ${ }^{\mathrm{TM}}$}

In $\mathrm{ABAQUS}{ }^{\mathrm{TM}}$, the material library allows most engineering materials to be modeled. For classical metal plasticity, it uses true-stress and true-plastic-strain to define the elastic-plastic material model. Young's modulus, yield stress and true-stress true-plastic-strain data of uniaxial test are important parameters in such finite element simulations.

\section{Review of stress-strain model}

\section{True stress and true strain}

For a uniaxial tensile or compressive test, the engineering stress-strain is defined as, 


$$
\varepsilon_{e}=\frac{l-l_{0}}{l_{0}} \quad \sigma_{e}=\frac{P}{A_{0}}
$$

Where $l$ is the deformed length and $l_{0}$ is the original length, $P$ is applied load, $A_{0}$ is original cross-section area. The $\sigma_{\mathrm{e}}-\varepsilon_{\mathrm{e}}$ plot is often called stress-strain curve.

True stress-strain was suggested by P. Ludwik (1909) ${ }^{[12]}$, and is now widely used in finite element analyses,

$$
\begin{aligned}
& \varepsilon=\int_{l_{0}}^{l} \frac{d l}{l}=\ln \left(\frac{l}{l_{0}}\right)=\ln \left(1+\varepsilon_{e}\right) \\
& \sigma=\frac{P}{A}=\frac{P A_{0} l_{0} l}{A_{0} l_{0} l A}=\frac{P}{A_{0}} \frac{A_{0} l_{0}}{A l} \frac{l}{l_{0}}=\sigma_{e}\left(1+\varepsilon_{e}\right) \frac{A_{0} l_{0}}{A l}=\sigma_{e}\left(1+\varepsilon_{e}\right)
\end{aligned}
$$

Under the condition of neglecting the elastic deformation and very small permanent volume change during a tensile test, i.e., $A_{0} l_{0}=A l$ ( or $\frac{A_{0}}{A}=\frac{l}{l_{0}}=\frac{1}{l / l_{0}}$, before necking). So the true stress is written as,

$$
\sigma=\sigma_{e}\left(1+\varepsilon_{e}\right)
$$

Maximum/ultimate tensile strength $\left(\sigma_{\mathrm{UTS}}\right)$ is calculated,

$$
\sigma_{U T S}=\frac{P_{\max }}{A_{0}}=\frac{\sigma}{e^{\varepsilon}}
$$

As pointed out by Ludwik, ultimate strength means that at this point the uniform extension of test piece cease. But in the true stress-strain curve, it has no difference from any other stresses.

In the stress-strain curve, at maximum load, $\frac{d \sigma_{e}}{d \varepsilon_{e}}=0, \sigma_{e}=\frac{\sigma}{1+\varepsilon_{e}}$

The derivative of Equation (3-6) results, $\frac{d \sigma}{d \varepsilon}=\frac{\sigma}{1+\varepsilon}$

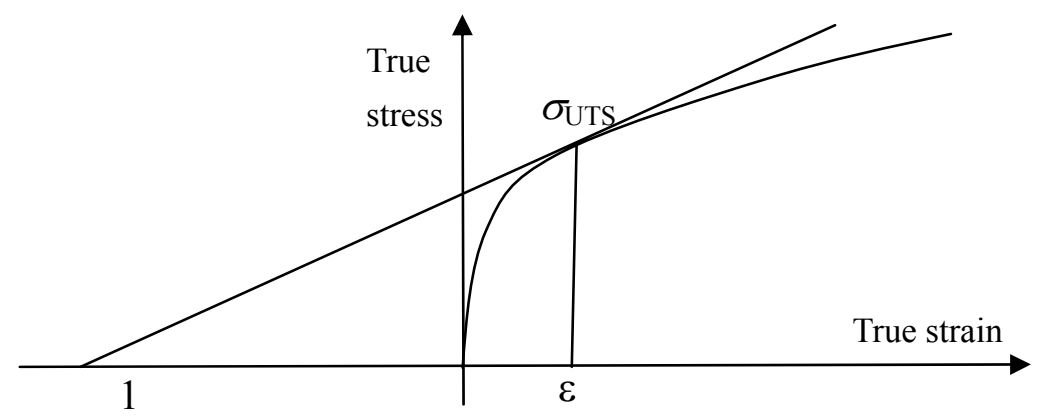

Figure 3.1 Ultimate tensile strength and true stress-strain curve 


\section{Stress-strain model}

There is no universal stress-strain model to fit all the materials. Different stress-strain models are used for different materials. For some material, a power function may be sufficient to describe the stress-strain relation $\left(\mathrm{Nadai}^{[12]}\right)$,

$$
\sigma=\sigma_{1} \varepsilon^{n}
$$

W.F. Chen ${ }^{[13]}$ categorized true-stress-strain curves into four idealized categories, i.e. (a) elastic-perfect plastic model (b) elastic-linear work-hardening model (c) elastic-power (Original: exponential) hardening model (d) Ramberg-Osgood model.

\section{(a) Elastic-perfect plastic model}

$$
\begin{array}{ll}
\varepsilon=\frac{\sigma}{E} & \text { for } \sigma<\sigma_{0} \\
\varepsilon=\frac{\sigma}{E}+\lambda & \text { for } \sigma=\sigma_{0}
\end{array}
$$

$\lambda$ is a scalar to be determined and is greater than 0 .

(b) Elastic-linear work-hardening model (Bi-Linear Model)

$$
\begin{array}{ll}
\varepsilon=\frac{\sigma}{E} & \text { for } \sigma \leq \sigma_{0} \\
\varepsilon=\frac{\sigma_{0}}{E}+\frac{1}{E_{t}}\left(\sigma-\sigma_{0}\right) & \text { for } \sigma>\sigma_{0}
\end{array}
$$

In this model, the strain-hardening is linearized and $E_{t}$ is the tangent modulus.

\section{(c) Elastic-Power hardening model}

$$
\begin{array}{ll}
\sigma=E \varepsilon & \text { for } \sigma \leq \sigma_{0} \\
\sigma=k \varepsilon^{n} & \text { for } \sigma>\sigma_{0}
\end{array}
$$

$k$ and $n$ are two characteristic constants of the material. They are determined to best fit the experimentally obtained curve. The power expression should be used only in the strain-hardening range. Two parts are continuous at yielding point. This model can also be written in different forms,

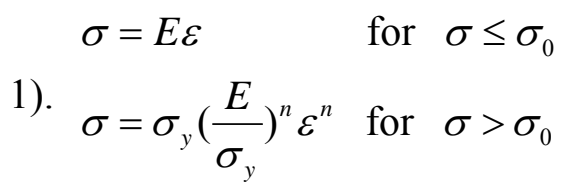

Compare Equation (3-11-1) and (3-11-2), $k=\sigma_{y}\left(\frac{E}{\sigma_{y}}\right)^{n}$ 


$$
\begin{array}{cc}
\sigma=E \varepsilon & \text { for } \sigma \leq \sigma_{0} \\
\sigma=\sigma_{y}\left(\frac{\varepsilon}{\varepsilon_{\mathrm{y}}}\right)^{\mathrm{n}} & \text { for } \quad \sigma>\sigma_{0}
\end{array}
$$

\section{(d) Ramberg-Osgood model}

$$
\varepsilon=\frac{\sigma}{E}+a\left(\frac{\sigma}{b}\right)^{n}
$$

Where $a, b$ and $n$ are material constants.

This model separates the elastic strain and plastic strain. Compared with other models, more parameters (three) allow better curve fitting of the real stress-strain curve.

Rewrite Equation (3-12) as $\varepsilon-\frac{\sigma}{E}=a\left(\frac{\sigma}{b}\right)^{n}$, and define plastic strain as $\varepsilon_{p}=\varepsilon-\frac{\sigma}{E}$,

$$
\begin{aligned}
& \varepsilon_{p}=a\left(\frac{\sigma}{b}\right)^{n} \text { or } \\
& \sigma=b\left(\frac{\varepsilon_{p}}{a}\right)^{\frac{1}{n}}=b\left(\frac{\varepsilon_{p}}{a}\right)^{n^{\prime}}
\end{aligned}
$$

This implies that true-stress true-plastic-strain has a power law relation in Ramberg-Osgood model. It should be noted that in Ramberg-Osgood model, the $n^{\prime}=1 / n$ is comparable with the $n$ in the elastic-power hardening model. In Ramberg-Osgood model, $n \geq 1$, while in the elastic-power hardening model, $n \leq 1$.

For most metal alloys, the first three models are frequently used in numerical simulations. In this research work, both elastic-linear work hardening and elastic-power hardening are used for the indentation finite element analyses.

\section{Classical metal elastic-plastic analysis in ABAQUS}

\section{Input of material mechanical properties}

For classical metal plasticity, ABAQUS ${ }^{\mathrm{TM}}$ only accepts true stress and true plastic strain data input. Between consecutive input data points, ABAQUS ${ }^{\mathrm{TM}}$ uses piece-wise linear interpolation to obtain intermediate data. Thus no matter what material model has been chosen, all the stress-strain inputs are similar. For elastic perfect plastic material, Young's modulus E, Poisson's ratio $v$, and yield stress $\sigma_{y}$ are input using the following commands,

*elastic

$E, v$

*plastic

$\sigma_{y}, \mathbf{0 . 0}$ 

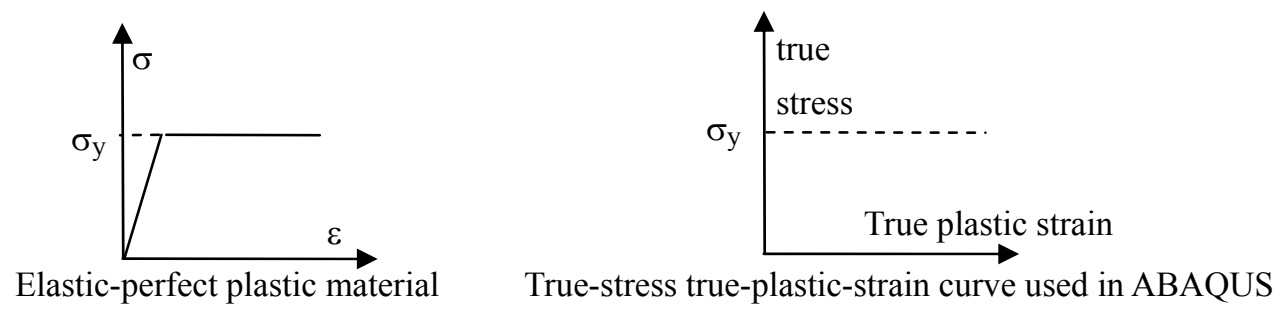

Figure 3.2 Elastic-perfect plastic material used in ABAQUS

For material with strain hardening, the true plastic strain is calculated from the true strain using following equation,

$$
\varepsilon^{p l}=\varepsilon^{\text {true }}-\varepsilon^{\text {elastic }}=\varepsilon^{\text {true }}-\sigma^{\text {true }} / E
$$

Use Equation (3-2), (3-4) and (3-14) to convert the engineering stress strain data to true stress and true plastic strain data as shown in Table 3.1. Finally, the input data are like this,

*elastic

E, $v$

*plastic

$782.4, \quad 0.0$

$1432.8,0.174241$

ABAQUS ${ }^{\mathrm{TM}}$ interpolates input data linearly between consecutive data points and assumes that the response is constant outside the range defined by the input data as shown in Figure 3.3.

Table 3.1 Stress and strain conversions for IN783

\begin{tabular}{|c|c|c|c|c|}
\hline Nominal Stress & Nominal Strain & True Stress & True Strain & True Plastic Strain \\
\hline Yield strength: 779 & 0.004394 & $\mathbf{7 8 2 . 4}$ & 0.004413 & $\mathbf{0 . 0}$ \\
\hline Ultimate Strength: 1194 & 0.20 & $\mathbf{1 4 3 2 . 8}$ & 0.182322 & $\mathbf{0 . 1 7 4 2 4 1}$ \\
\hline
\end{tabular}

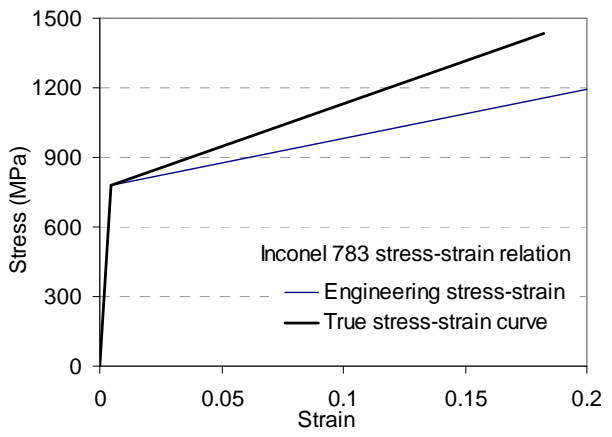

Elastic-Linear work hardening material

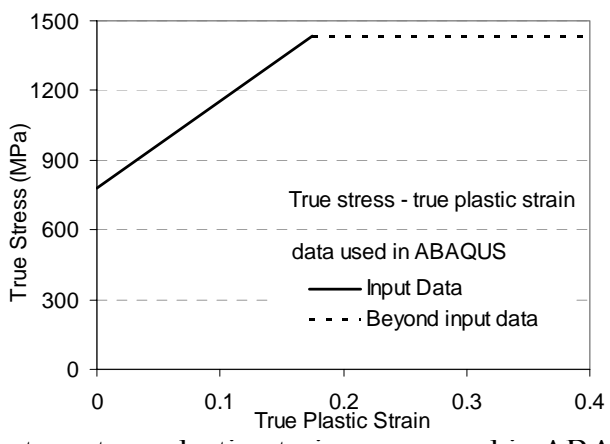

True-stress true-plastic-strain curve used in ABAQUS

Figure 3.3 Elastic linear work hardening material used in ABAQUS

\section{Element selection due to the shear locking and volume locking}

Elastic-plastic finite element analyses are very complex and proper elements must be chosen for different applications in order to obtain accurate simulation results. Incompressible 
plastic deformation adds kinematic constraints to the elements, and if the elements cannot solve all of these constraints, they will suffer from volumetric locking which causes their response to be too stiff. Modeling this effect places some severe restrictions on the type of the elements that can be used in elastic-plastic simulations.

Fully integrated, second-order, solid elements are very susceptible to volumetric locking when modeling incompressible material behavior and should not be used in elastic-plastic simulations. Instead, fully integrated, first-order, solid elements do not suffer from volumetric locking, and reduced-integration, solid elements can also be used for most elastic-plastic simulations. But second-order, reduced-integration elements should be used with caution if the strain exceeds $20 \sim 40 \%$ because at this magnitude they can suffer from volumetric locking. This can be reduced with mesh refinement. In the case that you have to use fully-integrated, second-order elements, use hybrid elements which are designed to model incompressible behavior in ABAQUS ${ }^{\mathrm{TM}}$, but it is more computationally expensive.

Unfortunately, fully integrated, first-order elements may suffer from shear locking. Usually shear locking occurs when these elements are subjected to bending. In such a case, the numerical formulation of the elements gives rise to shear strains that do not really exist, the so-called parasitic shear. First-order, reduced-integration elements are immunized from shear locking, but hourglassing can be a problem in stress/displacement analysis since they have only one integration point. These elements are possible to distort in such a way that the strains calculated at the integration point are all zero, which in turn, leads to uncontrolled distortion of the mesh. Fortunately, ABAQUS ${ }^{\mathrm{TM}}$ has the hourglassing control for first-order reduced-integration elements, but they should be used with reasonably fine mesh. Also, first-order elements are recommended when large strains or very high strain gradients are expected $^{[14]}$.

\section{Additional considerations for indentation contact modeling}

For an indentation process, contact modeling is inevitable except for 1D finite element simulation. In ABAQUSTM, there are two methods to model this contact problem, i.e. surface-based contact pair or contact-elements-based. Surfaces-based contact pair method is used in this research work to avoid pre-assumption of contact pressure distribution.

To model the indentation process using surface-base contact pair method, first, define surfaces that potentially contact with each other; secondly, define the Master-Slave surfaces which interact with each other; thirdly, define the surface mechanical interaction models that govern the behavior of the surfaces when they are in contact, such as small or finite sliding, friction coefficient and pressure-clearance relationship.

Using surfaces-based contact method, the indenter is modeled as either rigid or deformable body. Thus the influence of the indenter rigidity (rigid or deformable) can also be studied in the simulation. This modeling method is also applicable for 3D modeling. 
For contact analysis, it is better to use the first-order elements for those parts of a model that will form a slave surface. Second-order elements can sometimes cause problems in contact simulations, because of the way these elements calculate consistent nodal loads for a constant pressure $^{[14]}$.

Volumetric locking and shearing locking should also be avoided since the material underneath the indenter may undergo large strain and bending deformation. As we have known, first-order, fully integrated elements may suffer from shearing locking and second-order; fully integrated elements may suffer from volumetric locking; and second-order, reduced-integration can suffer from volumetric locking at strain larger than $20 \%$. Since first-order, reduced-integration elements do not suffer from these problems, and ABAQUS has built-in hourglassing control for this type of elements, for 2D indentation simulation, axisymmetric, first-order, reduced-integration elements are used (CAX4R).

\subsection{Finite element models for indentation analyses}

From the literature and the results which will be discussed in the experiments, the deformation fields $(u, v$ or $w)$ are limited in a $3 \mathrm{~mm}$ radius circle for indentation loads under $1000 \mathrm{~N}$. In this research, an $8 \mathrm{~mm}$ radius by $8 \mathrm{~mm}$ height cylinder specimen is modeled to study the indentation process. For a spherical/ball indenter, under axially symmetric loading conditions, the 2D FEA is adequate to study the indentation process instead of creating a complex 3D model. Thus in this research, both residual field analyses and in-situ elastic recovery analyses are investigated using this model with either a $1.6 \mathrm{~mm}$ or $2 \mathrm{~mm}$ diameter indenters. It is also assumed that the bulk material is homogeneous, and the indentation load is axisymmetric. Except for the rigid surface, all meshes use the first-order, axisymmetric element with reduced-integration (CAX4R). The surface-based contact-pair method is used to model the indentation process.

\section{Axisymmetric indentation model}

As shown in Figure 3.4, the axisymmetric 2D model in ABAQUS ${ }^{\mathrm{TM}}$ is described in the cylindrical polar coordinate system $(r, z$ and $\theta$ ). Coordinate 1 represents $r$, and coordinate 2 represents $z$ direction, and the model is laid in the $\theta=0$ plane. Degree of freedom (DOF) 1 represents displacement $u_{r}$, and DOF 2 represents $u_{z}$. Generalized axisymmetric elements with twist may use an additional DOF 5 corresponding to the twist angle. In the current indentation simulations, only DOF 1 and DOF 2 are active.

It is necessary to setup the boundary conditions for the nodes on the symmetric axis since ABAQUS ${ }^{\mathrm{TM}}$ does not automatically apply any boundary conditions to axisymmetric model. Without these restrictions, nodes located on the axis may across the symmetric axis, violating the principle of compatibility. Also for point loads and moments, they should be given as the value integrated around the circumference (that is the total value on the ring). In the literature, it has been shown that the outside boundary condition has negligible effects on the simulation 
results for large enough specimen model.

Either rigid indenter or deformable indenter can be modeled to study the effect of the indenter rigidity. For rigid indenter, load-depth curve can be directly obtained from ABAQUS ${ }^{\mathrm{TM}}$ history data of the reaction force of the indenter and down/up movement of the indenter. For deformable indenter, adding up all the reaction force may be necessary.

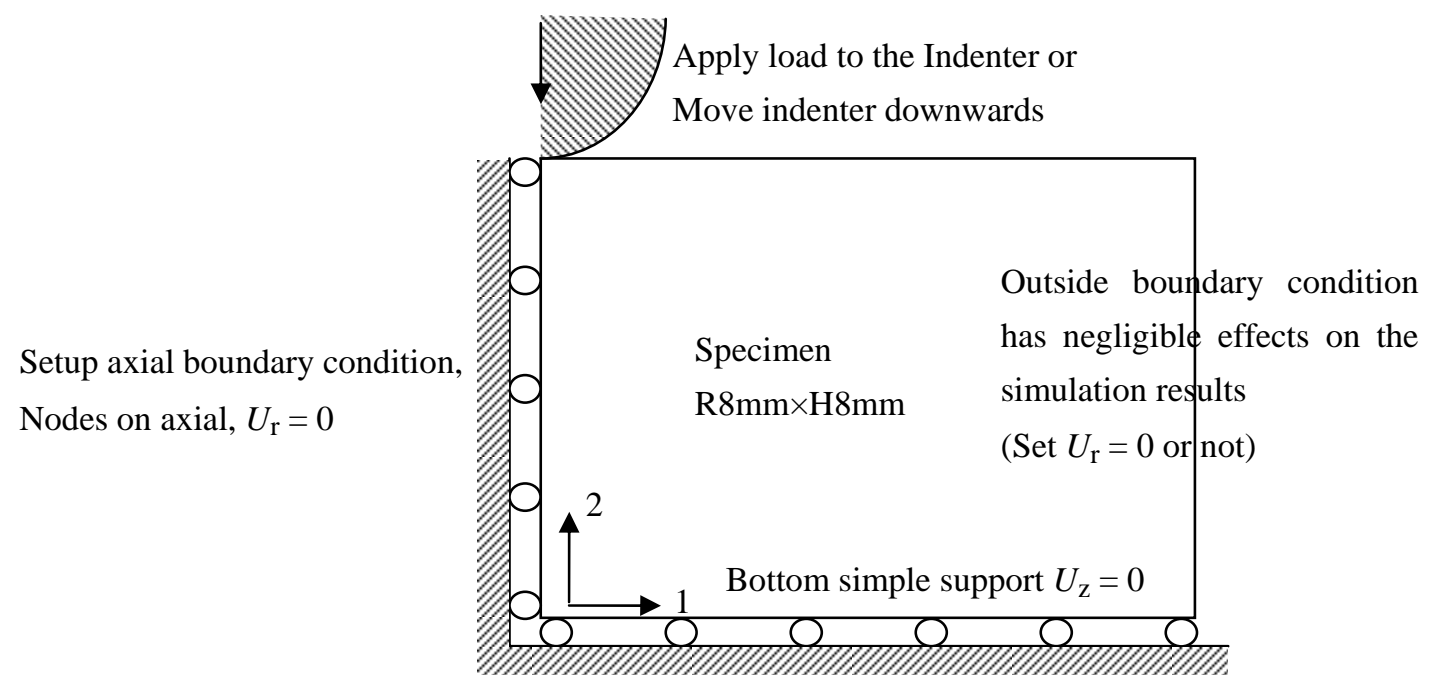

Figure 3.4 Axisymmetric indentation model using a spherical indenter

\section{Mesh design: Residual deformation analysis}

For indentation-induced residual deformation analysis, the finite element model is corresponding to a 1.6mm-diameter tungsten carbide ball indenter and IN783 specimen.

The 1.6mm-diameter tungsten carbide ball indenter is simplified as a rigid surface. The IN783 specimen has standard heat treatment $\left(1120^{\circ} \mathrm{C} / 1 \mathrm{hr} / \mathrm{AC}+845^{\circ} \mathrm{C} / 8 \mathrm{hrs} / \mathrm{AC}+720^{\circ} \mathrm{C} / 8 \mathrm{hrs} 50^{\circ} \mathrm{C} / \mathrm{hr}\right.$ $620^{\circ} \mathrm{C} / 8 \mathrm{hrs} / \mathrm{AC}$ ). For this material, a simplified bi-linear isotropic stress-strain relation is used as shown in Figure 3.3 and Table 3.1. Frictionless $(f=0)$ and an estimated friction coefficient ( $f=0.3$ ) are used to study the friction influence.

The $8 \mathrm{~mm} \times 8 \mathrm{~mm} 2 \mathrm{D}$ model is then meshed using the first-order, axisymmetric elements (CAX4R) as shown in Figure 3.5. It consists of 11031 CAX4R elements. Fine mesh is used directly underneath the indenter and the mesh gradually changes from fine to coarse using trapezoidal transition elements from region 1 to 4 . The smallest element (in region 1) is $2 \mu \mathrm{m} \times 2 \mu \mathrm{m}$ which covers the entire possible contact region and provides good spatial resolution for determining critical indentation parameters. For region 2, 3, and 4, the element size is $32 \mu \mathrm{m} \times 32 \mu \mathrm{m}, 64 \mu \mathrm{m} \times 64 \mu \mathrm{m}, 256 \mu \mathrm{m} \times 256 \mu \mathrm{m}$, respectively. 


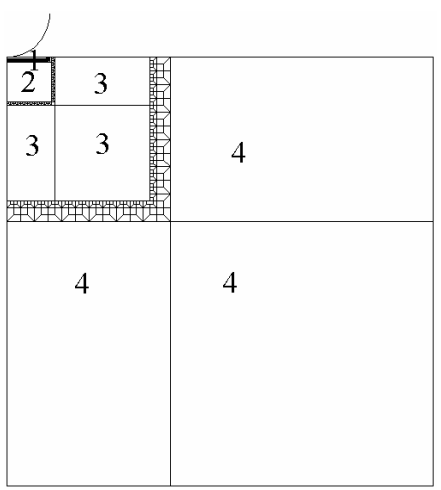

(a) Transition region

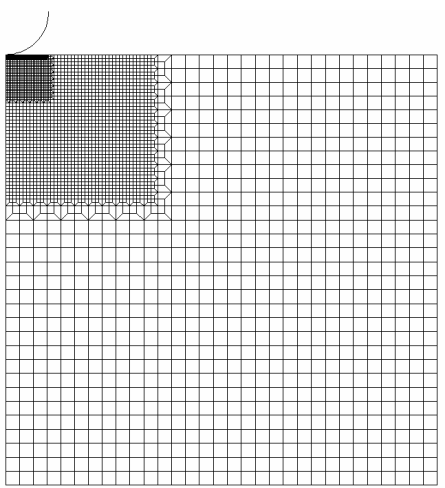

(b) Specimen mesh

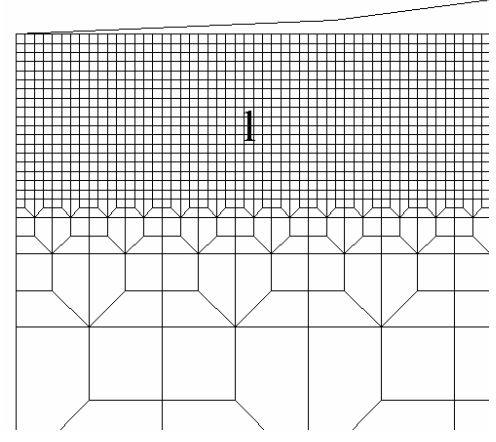

(c) Mesh underneath the indenter

Figure 3.5 Finite element mesh design for residual deformation analyses

\section{Mesh design: Elastic recovery analysis}

In elastic recovery analyses, relative coarse mesh is used compared with mesh used in residual deformation analyses. This part focuses on the elastic recovery analyses and procedure development for Young's modulus calculation. Since the TIM (chapter 5) uses either BK7 optical glass $(\mathrm{E} \sim 81 \mathrm{GPa})$ or/and sapphire $(\mathrm{E} \sim 344 \mathrm{GPa})$ half ball indenters which have much lower Young's modulus compared with tungsten carbide indenter, it is necessary to study the elastic indenter effects on the indentation process. Both IN783 superalloy and A16061-T0 alloy are tested in the experiments, but only the combinations as shown in Table 3.2 are used for finite element analyses which cover the extreme cases.

The $8 \mathrm{~mm} \times 8 \mathrm{~mm} 2 \mathrm{D}$ specimen model uses the same mesh as shown Figure 3.6, which consists of 6481 first-order axisymmetric elements (CAX4R). For the $2 \mathrm{~mm}$-diameter indenter, only deformable indenter is meshed using the CAX4R element. Fine mesh is obtained for possible contact region and the mesh gradually changes from fine to coarse using trapezoidal transition elements. The smallest specimen element is $16 \mu \mathrm{m} \times 16 \mu \mathrm{m}$.

Table 3.2 Material matrix for elastic recovery analyses

\begin{tabular}{|l|l|}
\hline \multicolumn{1}{|c|}{ Specimen(IN783) } & \multicolumn{1}{c|}{ Indenter } \\
\hline $\mathrm{E}=177.3 \mathrm{GPa}$ & Rigid indenter \\
\cline { 2 - 2 }$\sigma_{\mathrm{y}}=779 \mathrm{MPa}$ & Deformable indenter \\
$\sigma_{\text {ult }}=1194 \mathrm{MPa}$ & (BK7 optical glass) \\
(at strain $=0.20)$ & $\mathrm{E}=81 \mathrm{GPa}$ \\
$v=0.31$ & $v=0.206$ \\
\hline
\end{tabular}




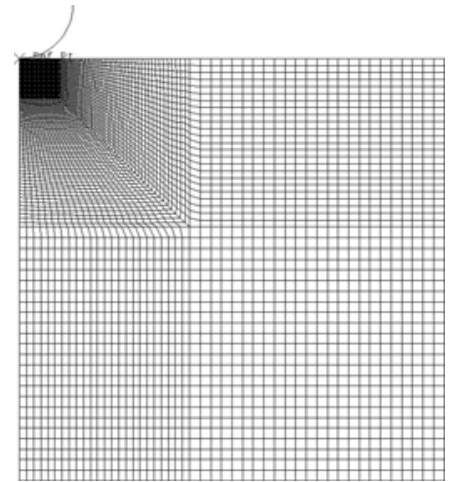

(a) Specimen mesh

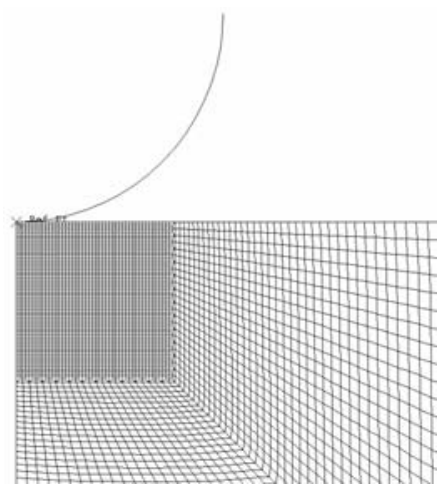

(b) Rigid indenter local mesh

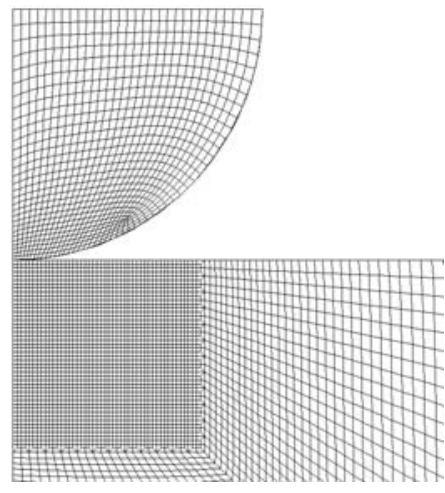

(c) Deformable indenter local mesh

Figure 3.6 Finite element mesh design for elastic recovery analyses

\subsection{Validation of indentation model}

The validation of the indentation FEA model is verified for both elastic and elastic-plastic materials. In the elastic case, the theoretical solution is compared with finite element results. And in the elastic-plastic case, surface deformation from experimental measurements is directly compared with the finite element results. Load-depth relation and unloading characteristics are also compared with conclusions from the literature.

\section{Elastic indentation verification}

Pure elastic finite element simulations are conducted for elastic materials based on elastic material properties of the IN783 superalloy and Aluminum alloy. Finite element results are compared to the analytical solutions to check the validation of the indentation models.

For rigid indenter, the analytical load-depth relation for ball indentation on elastic material is described as ${ }^{[30]}$,

$$
P=\frac{4}{3} \cdot \frac{\sqrt{R}}{k_{0}} h^{3 / 2}
$$

That is,

$P=2.920 h^{1.5}$ for a $1.6 \mathrm{~mm}$-diamter rigid indenter and elastic Aluminum, and

$P=7.397 h^{3 / 2}$ for a $1.6 \mathrm{~mm}$-diameter rigid indenter and elastic IN783.

Where $P$ in Newton and $h$ in $\mu \mathrm{m}$.

Figure 3.7 and Figure 3.8 show the load-depth curve from the finite element analyses. All the results are comparable with the analytical solution. Further more, Young's moduli are calculated from curve fitting coefficients as shown in Table 3.3. 
Table 3.3 Elastic verification results

\begin{tabular}{|l|c|c|c|c|}
\hline \multirow{2}{*}{$\begin{array}{c}\text { Elastic Indentation } \\
\text { Depth }(\mu \mathrm{m})\end{array}$} & \multicolumn{2}{|c|}{ E=69.0GPa, $\boldsymbol{v}=\mathbf{0 . 3 3 ( A l u m i n u m )}$} & \multicolumn{2}{c|}{ E=177.3GPa, $\boldsymbol{v}=\mathbf{0 . 3 1 ( I N 7 8 3 )}$} \\
\cline { 2 - 5 } & FEM results & Error $(\%)$ & FEM results & Error (\%) \\
\hline $\mathbf{1 0}$ & 68.7 & -0.4 & 176.5 & -0.5 \\
\hline $\mathbf{1 0 0}$ & 67.4 & -2.3 & 172.8 & -2.5 \\
\hline
\end{tabular}

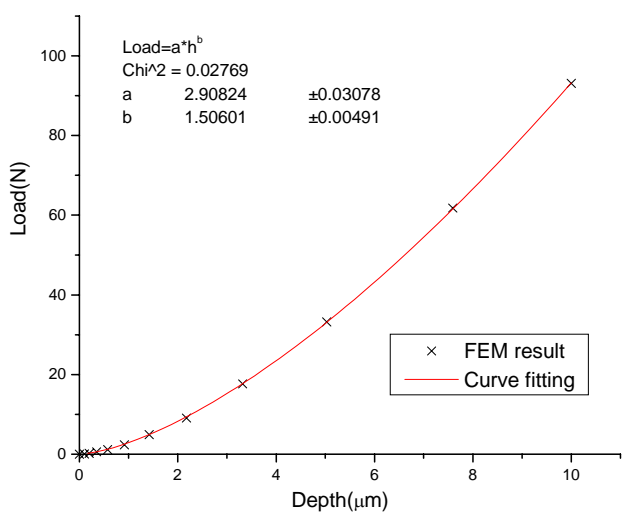

Max. Depth $10 \mu \mathrm{m}$

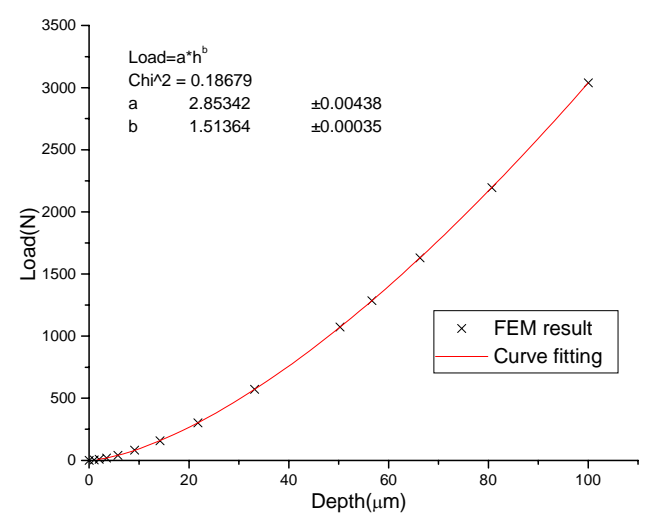

Max. Depth $100 \mu \mathrm{m}$

Figure 3.7 Load-depth curve for elastic Aluminum
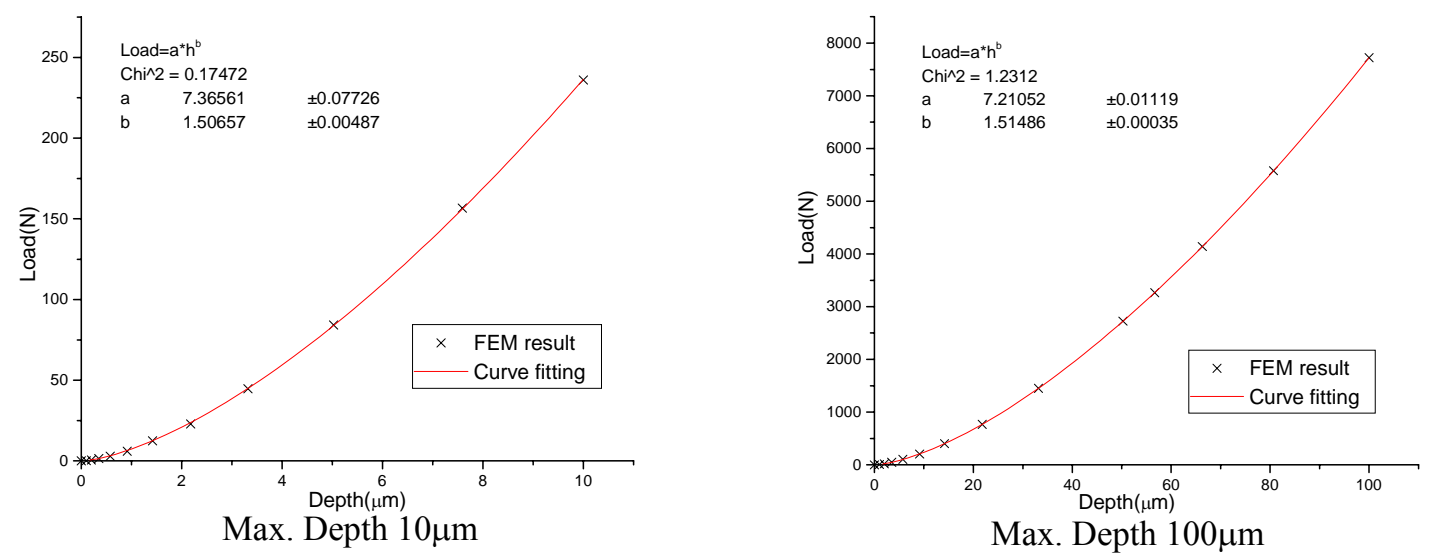

Figure 3.8 Load-depth curve for elastic IN783

As shown in Figure 3.9, the location of maximum von-Mises stress is consistent with the elastic solution which predicts the location of the maximum von-Mises stress is below the indenter, not directly underneath the indenter. 


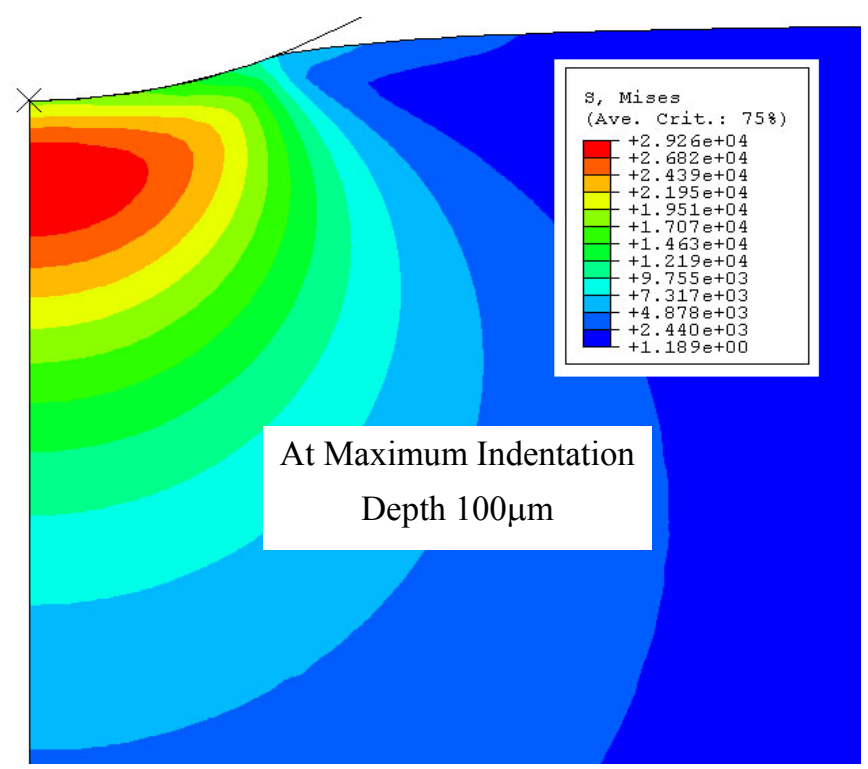

Figure 3.9 von-Mises stress distribution for elastic IN783 material

\section{Elastic-plastic indentation verification}

The verification of the elastic-plastic material is consider in the following four aspects for a $550 \mathrm{~N}$ indentation test, i.e. (1) the residual deformation fields, (2) contact radius, (3) load-depth curve and (4) elastic unloading.

Firstly, the residual fields from finite element simulation are compared with the experimental results for both in-plane radial residual displacement $u_{\mathrm{r}}$ and out-of-plane residual deformation $w$. As shown in Figure 3.10, they are comparable except at near the contact boundary, where the experimental results are smaller than finite element results. This is expected since there is no friction and damage of the tested material considered in the finite element model.

Secondly, the residual contact radius from the finite element analysis $(0.247 \mathrm{~mm})$ is comparable with the experimental result $(0.243 \mathrm{~mm})$. Figure 3.11 shows the load-depth curve of finite element analyses, and unloading slope can be used to calculate the Young's modulus with good accuracy as described in most literature. Finally, after the indentation load is released, even some of the material is still yielded; the actively yielding flag indicates that the unloading is indeed an elastic process as shown in Figure 3.12. 


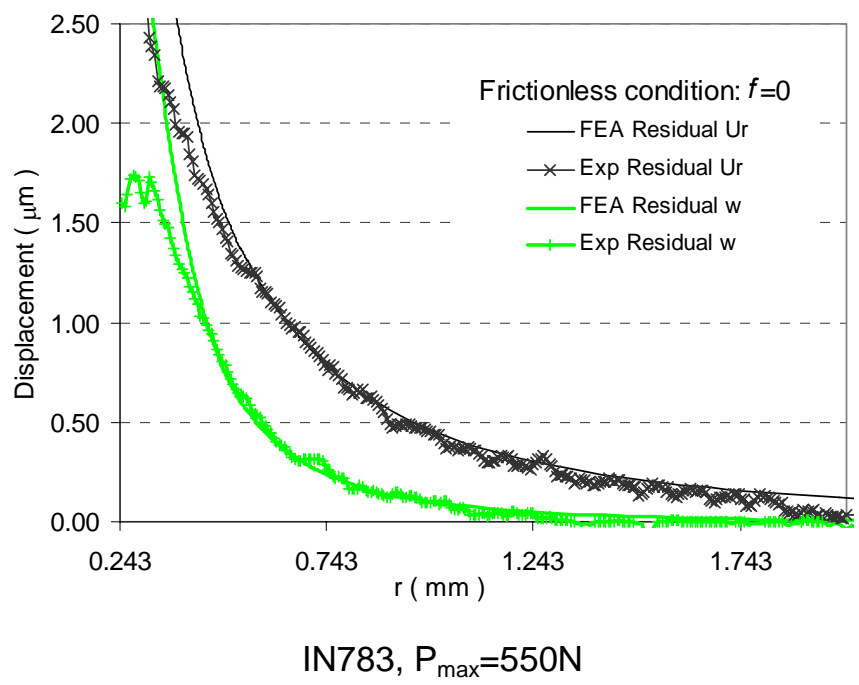

Figure 3.10 Comparison of residual deformation between FEA and experimental

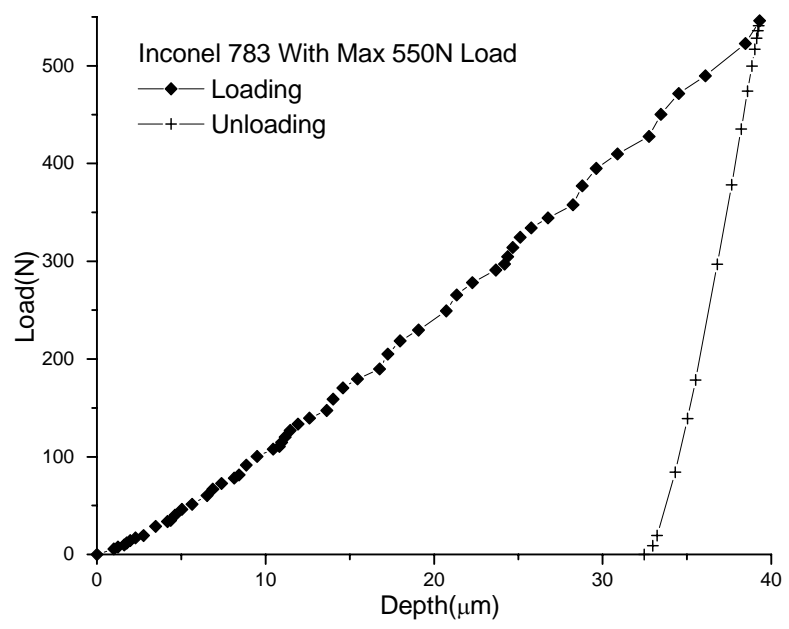

Figure 3.11 Load-depth curve of IN783 with maximum 550N indentation

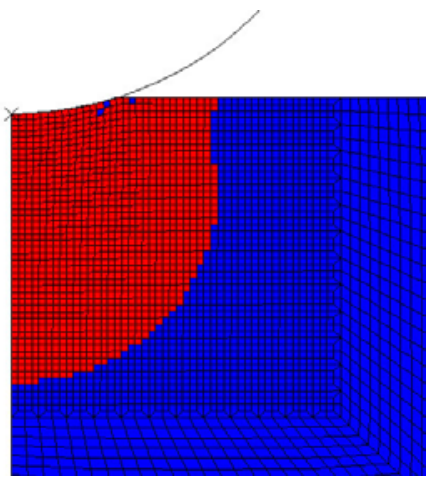

Actively yield zone before unloading

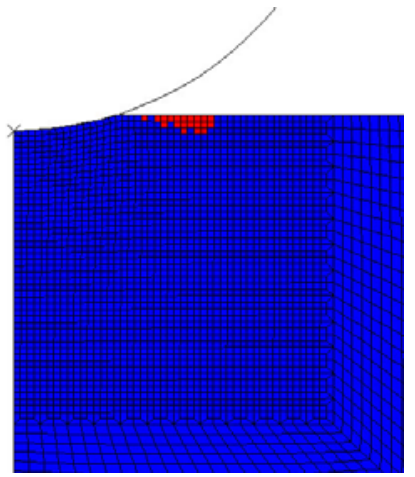

Actively yield zone during unloading

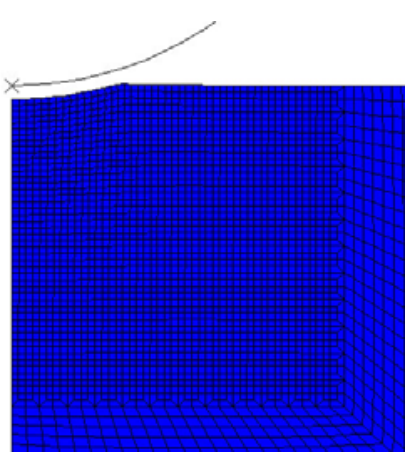

Fully unloading

Figure 3.12 Elastic recovery of indentation unloading 


\subsection{Surface deformation analysis}

In this section, the indentation-induced specimen surface deformation is analyzed to correlate to material mechanical properties.

\section{Top free surface stress-strain analysis: Yield strength}

Under cylindrical coordinate system, the small deformation elastic strain is defined as,

$$
\begin{aligned}
\varepsilon_{r r} & =\frac{\partial u_{r}}{\partial r} \\
\varepsilon_{\theta \theta} & =\frac{1}{r} \frac{\partial u_{\theta}}{\partial \theta}+\frac{u_{r}}{r} \\
\varepsilon_{z z} & =\frac{\partial u_{z}}{\partial z} \\
\varepsilon_{r \theta} & =\frac{1}{2}\left[\frac{1}{r} \frac{\partial u_{r}}{\partial \theta}+\frac{\partial u_{\theta}}{\partial r}-\frac{u_{\theta}}{r}\right] \\
\varepsilon_{r z} & =\frac{1}{2}\left[\frac{\partial u_{r}}{\partial z}+\frac{\partial u_{z}}{\partial r}\right] \\
\varepsilon_{\theta z} & =\frac{1}{2}\left[\frac{\partial u_{\theta}}{\partial z}+\frac{1}{r} \frac{\partial u_{z}}{\partial \theta}\right]
\end{aligned}
$$

If the material is still under elastic deformation, Hooke's law applies,

$$
\begin{aligned}
& \varepsilon_{r r}=\frac{1}{E}\left[\sigma_{r r}-v \sigma_{\theta \theta}-v \sigma_{z z}\right] \\
& \varepsilon_{\theta \theta}=\frac{1}{E}\left[\sigma_{\theta \theta}-v \sigma_{r r}-v \sigma_{z z}\right] \\
& \varepsilon_{z z}=\frac{1}{E}\left[\sigma_{z z}-v \sigma_{\theta \theta}-v \sigma_{r r}\right] \\
& \varepsilon_{r \theta}=\frac{1+v}{E} \sigma_{r \theta} \\
& \varepsilon_{r z}=\frac{1+v}{E} \sigma_{r z} \\
& \varepsilon_{\theta z}=\frac{1+v}{E} \sigma_{\theta z}
\end{aligned}
$$

Under the condition of axisymmetric indentation process, the indentation-induced deformation $(u, v, w)$ is also axisymmetric. The specimen top free surface displacements are $u_{\theta}=0, u_{r}=f(r)$ and $u_{z}=g(r)$. It is obvious that $\frac{\partial u_{r}}{\partial \theta}=0$ and $\varepsilon_{r \theta}=0$. On the top free surface, it is known that stress components $\sigma_{z z}, \sigma_{r z}$ and $\sigma_{\theta z}$ are zero. From Hook's law, it is obvious that $\varepsilon_{r} \theta$, $\varepsilon_{\theta z}$ and $\varepsilon_{r z}$ are all zero. So only $\varepsilon_{r r}, \varepsilon_{\theta \theta}$ and $\varepsilon_{z z}$ are non-zero strain components. 


$$
\begin{aligned}
& \varepsilon_{r r}=\frac{\partial u_{r}}{\partial r} \\
& \varepsilon_{\theta \theta}=\frac{u_{r}}{r} \\
& \varepsilon_{z z}=\frac{\partial u_{z}}{\partial z} \\
& \varepsilon_{r \theta}=\frac{1}{2 r} \frac{\partial u_{r}}{\partial \theta}=0 \\
& \varepsilon_{r z}=\frac{1}{2}\left[\frac{\partial u_{r}}{\partial z}+\frac{\partial u_{z}}{\partial r}\right]=0 \\
& \varepsilon_{\theta z}=\frac{1}{2 r} \frac{\partial u_{z}}{\partial \theta}=0 \\
& \varepsilon_{r r}=\frac{1}{E}\left[\sigma_{r r}-v \sigma_{\theta \theta}\right] \\
& \varepsilon_{\theta \theta}=\frac{1}{E}\left[\sigma_{\theta \theta}-v \sigma_{r r}\right] \\
& \text { and } \quad \varepsilon_{z z}=\frac{1}{E}\left[-v \sigma_{\theta \theta}-v \sigma_{r r}\right] \\
& \varepsilon_{r \theta}=\frac{1+v}{E} \sigma_{r \theta}=0 \\
& \varepsilon_{r z}=0 \\
& \varepsilon_{\theta z}=0
\end{aligned}
$$

From Equation (3-18), for top free surface, the non-zero components are $\varepsilon_{r r}, \varepsilon_{\theta \theta}, \varepsilon_{z z}, \sigma_{r r}$ and $\sigma_{\theta \theta}$. Then the Hooke's law is simplified as,

$$
\begin{aligned}
& \varepsilon_{r r}=\frac{\partial u_{r}}{\partial r} \\
& \varepsilon_{\theta \theta}=\frac{u_{r}}{r} \\
& \varepsilon_{z z}=\frac{-v}{E}\left[\sigma_{r r}+\sigma_{\theta \theta}\right] \\
& \sigma_{r r}=\frac{E}{1-v^{2}}\left[\varepsilon_{r r}+v \varepsilon_{\theta \theta}\right] \\
& \sigma_{\theta \theta}=\frac{E}{1-v^{2}}\left[\varepsilon_{\theta \theta}+v \varepsilon_{r r}\right]
\end{aligned}
$$

Thus the equivalent Von-Mises stress can be calculated,

$$
\sigma_{\text {Mises }}=\sqrt{\sigma_{r r}^{2}+\sigma_{\theta \theta}^{2}-\sigma_{r r} \sigma_{\theta \theta}}
$$

Using Equation (3-19) to (3-21), elastic von-Mises stress distribution can be calculated from the top free surface in-plane $\left(u_{r}\right)$ deformation. This relation is theoretically valid up to the point where material starts to yield, the so-called elastic-plastic boundary.

As shown in Figure 3.13, the surface residual stress (von-Mises) obtained directly from FEA results and the elastic solution calculated using FEA surface displacements and Equation (3-21) matched as expected in the elastic region. After material is yielded, they deviate and elastic solution can no long describe the surface residual stress. The location where surface material starts to yield is called elastic-plastic boundary (EPB, the knee point in Figure 3.13).

It should be emphasized that this relation can only apply in the elastic region (Beyond the elastic-plastic boundary). When von-Mises stress is larger than yield stress, this relation 
becomes invalid. This can be used to calculate the yield strength if the elastic-plastic boundary can be identified using other technique.

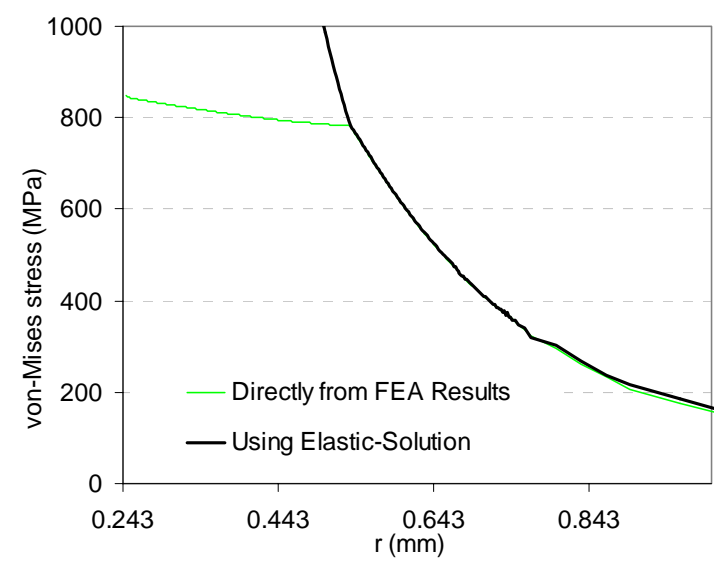

Figure 3.13 Comparison of surface von-Mises stress distribution

\section{Top free surface elastic recovery: Young's modulus}

Since the indentation unloading is an elastic process, the elastic solutions based on contact mechanics are applicable to characterize the unloading process. For indentations on pure elastic material, both the flat-end punch indentation and spherical indentation solutions are available.

For flat-end punch indentation ${ }^{[16]}$,

$u_{z}(\rho, 0)=\frac{1-v^{2}}{E} \frac{P}{\pi a} \sin ^{-1}\left(\frac{a}{\rho}\right), \rho>\mathrm{a}$

For spherical indentation ${ }^{[32,86]}$,

$u_{z}(\rho, 0)=\frac{3}{4} \frac{1-v^{2}}{E} \frac{P}{\pi a^{3}}\left[\left(2 a^{2}-\rho^{2}\right) \sin ^{-1}\left(\frac{a}{\rho}\right)+a \sqrt{\rho^{2}-a^{2}}\right], \quad \rho>\mathrm{a}$

Where $u_{z}$ is the elastic out-of-plane displacement, $\mathrm{P}$ is the applied load, $a$ is the contact radius and $\rho$ is the distance to the indentation center.

In an indentation test, the distribution of unloading-induced elastic recovery $(\Delta w)$ can be modeled by Equations (3-22) and (3-23) for either the flat-end punch or spherical indentation model.

For flat-end punch model,

$$
\Delta w(\rho, 0)=\frac{1-v^{2}}{E} \frac{\Delta P}{\pi a} \sin ^{-1}\left(\frac{a}{\rho}\right), \rho>\mathrm{a}
$$

For spherical indentation model, 


$$
\Delta w(\rho, 0)=\frac{3}{4} \frac{1-v^{2}}{E} \frac{\Delta P}{\pi a^{3}}\left[\left(2 a^{2}-\rho^{2}\right) \sin ^{-1}\left(\frac{a}{\rho}\right)+a \sqrt{\rho^{2}-a^{2}}\right], \quad \rho>\mathrm{a}
$$

Poisson's ratio and Young's modulus are the only mechanical properties involved in Equations (3-24) and (3-25). Thus Young's modulus can be determined from Equations (3-24) or (3-25) if Poisson's ratio is known.

To make the problem clear, both models can be transformed to linear relationship by re-writing Equations (3-24) and (3-25),

$$
\Delta w(t)=m t+b
$$

Where for the flat-end punch model,

$$
\begin{aligned}
& t=\sin ^{-1}\left(\frac{a}{\rho}\right) \\
& m=\frac{1-v^{2}}{E} \frac{P}{\pi a}
\end{aligned}
$$

And for the spherical indentation,

$$
\begin{aligned}
& t=\left[\left(2 a^{2}-\rho^{2}\right) \sin ^{-1}\left(\frac{a}{\rho}\right)+a \sqrt{\rho^{2}-a^{2}}\right] \\
& m=\frac{3}{4} \frac{1-v^{2}}{E} \frac{P}{\pi a^{3}}
\end{aligned}
$$

The constant $b$ is used to compensate the datum difference in experimental data.

By converting experimental $\Delta w \sim \rho$ data to $\Delta w \sim t$ relation, $m$ is determined from linear curve fitting of the $\Delta w \sim t$ data. Then using the measured load $P$ and contact radius $a$, Young's modulus is be determined according to Equation (3-28),

$$
\begin{aligned}
& E=\left(1-v^{2}\right) \frac{P}{\pi a} \frac{1}{m} \\
& E=\frac{3}{4}\left(1-v^{2}\right) \frac{P}{\pi a^{3}} \frac{1}{m}
\end{aligned}
$$




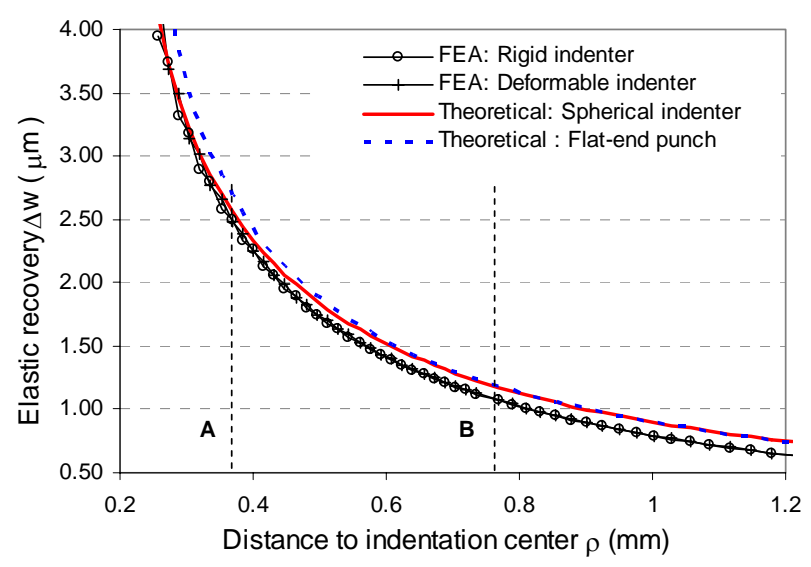

Figure 3.14 Comparison of elastic recovery $\Delta w$

The experimental measurement of elastic recovery $(\Delta w)$ will be discussed later in Chapter 5. The following discussions are only based on the finite element results using material mechanical properties as shown in Table 3.2.

Figure 3.14 shows the comparative results of elastic c recovery $(\Delta w)$ between FEA results and theoretical solutions. For both of the spherical and flat-end punch solutions, the FEM results match well with the analytical solutions except very near the contact boundary where the difference is noticeable. This is expected that due to the plastic deformation, the region near the contact radius may deform irregularly ${ }^{[12,22]}$. Converting $\Delta w \sim \rho$ relation to $\Delta w \sim t$ relation using Equation (2-27), the linearity showing in Figure 3.15 indicates the region of validity (i.e. From A to B) of using the elastic recovery solution for Young's modulus determination.

Specifically, Young's modulus is calculated using Equation (2-29) where $m$ is the slope of the linear portion of $\Delta w \sim t$ plots between A and B in Figure 3.15. Table 3.4 shows that the error for both flat-end and spherical models are all less than $5 \%$ which means that either model can be used to calculate Young's modulus with good accuracy.

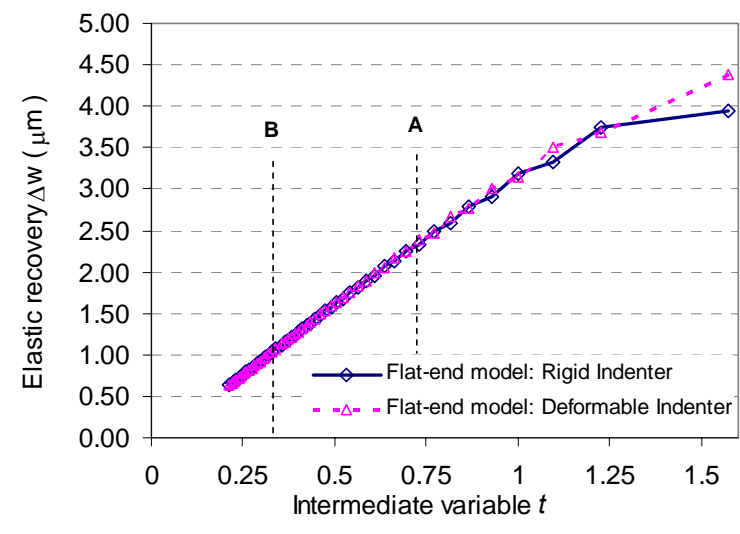

(a) $\Delta w \sim t$ relation using flat-end punch model $\mathrm{A}=1.5 \mathrm{a}=0.384 \mathrm{~mm}, \mathrm{~B}=0.7$

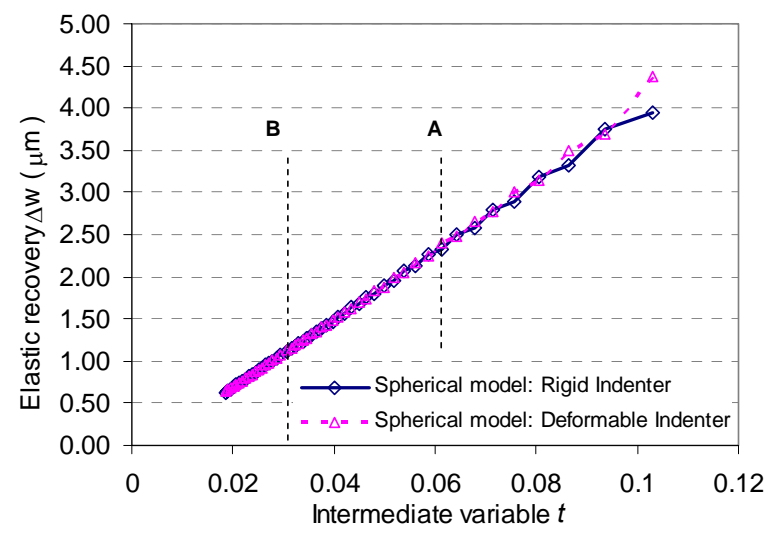

(b) $\Delta w \sim t \quad$ relation using spherical indenter model .768mm, $P_{\max }=550 \mathrm{~N}, P_{\min }=0$

Figure 3.15 FEA elastic recovery 
Table 3.4 Error of Young's modulus calculation

\begin{tabular}{|l|c|c|}
\hline & Rigid Indenter & Deformable Indenter \\
\hline Flat-end model & $\leq 0.6 \%$ & $\leq 3.3 \%$ \\
\hline Spherical model & $\leq 0.2 \%$ & $\leq 1.7 \%$ \\
\hline
\end{tabular}

\subsection{Residual deformation analyses for IN783}

Detailed residual deformation analyses are carried out to investigate the relation between the surface residual displacements and yield strength, elastic-plastic boundary (EPB) or contact radius. The 2D finite element model shown in Figure 3.5 and IN783 material properties listed in Table 3.2 are used for the residual deformation analyses. Corresponding to the experiments, the finite element residual deformation analyses were conducted as scheduled in Table 3.5. The analyses in this part are only focused on the residual deformation fields, but other indentation parameters can also be investigated as well. The experimental results will be discussed in next chapter.

Table 3.5 Indentation loading schedule

\begin{tabular}{|c|c|c|c|c|c|c|c|c|c|c|}
\hline Test \# & $\mathbf{1}$ & $\mathbf{2}$ & $\mathbf{3}$ & $\mathbf{4}$ & $\mathbf{5}$ & $\mathbf{6}$ & $\mathbf{7}$ & $\mathbf{8}$ & $\mathbf{9}$ & $\mathbf{1 0}$ \\
\hline Load(N) & 100 & 200 & 300 & 400 & 550 & 610 & 710 & 820 & 900 & 1000 \\
\hline
\end{tabular}

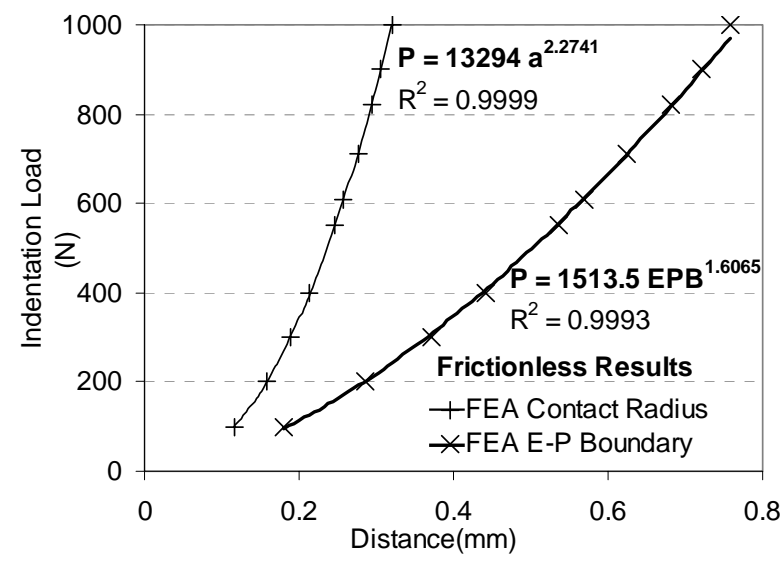

Figure 3.16 Load vs. contact-radius and elastic-plastic boundary relation 


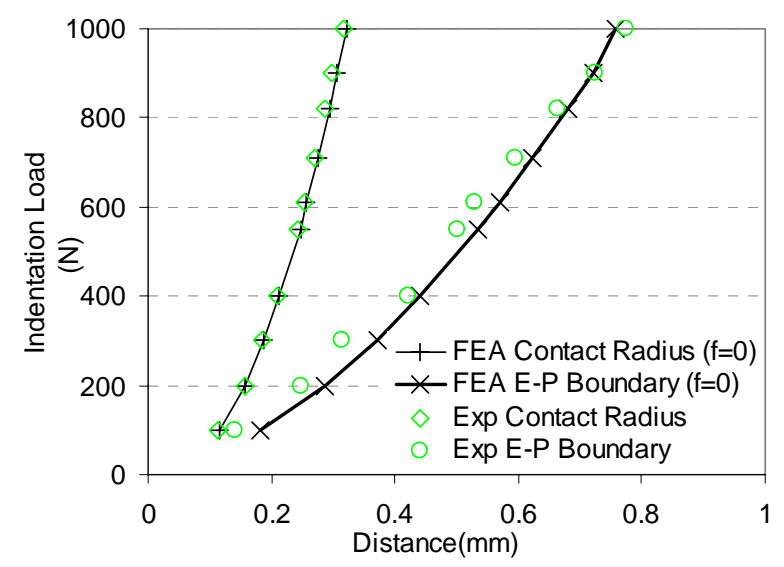

Figure 3.17 Frictionless FEA results compared with experimental results

Figure 3.16 shows the results of contact radii and elastic-plastic boundaries directly obtained from the frictionless finite element simulations of IN783 superalloy. It is not surprised that load and contact radius follow power law form since Meyer's law already shows such results. What surprised us is that the load elastic-plastic boundary also follows a similar power law form, in which both coefficients are smaller than that in a load-contact radius relation. The results directly from experiments (in Chapter 4) have confirmed this observation. As shown in Figure 3.17, the FEA frictionless results matched well with the experimental results. Two possible reasons may contribute the small difference, friction coefficients and Lüder strain effects. Lüder strain is not considered in this simulation. It is well known that there is almost no strain hardening during the development of Lüder band, so it basically weakens the material at the initial yielding stage. Thus it is reasonable to believe that Lüder strain effects will expend the contact radius or elastic-plastic boundary.

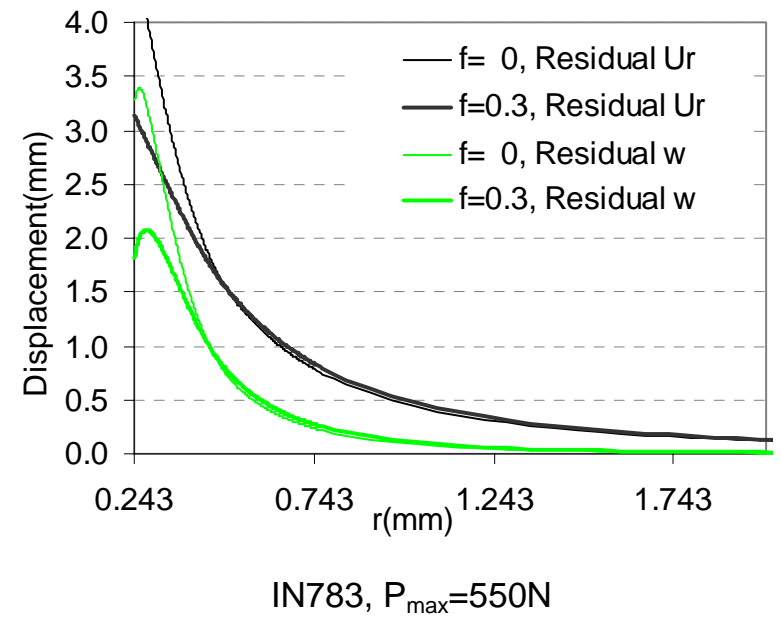

Figure 3.18 Friction effects on surface residual deformation 


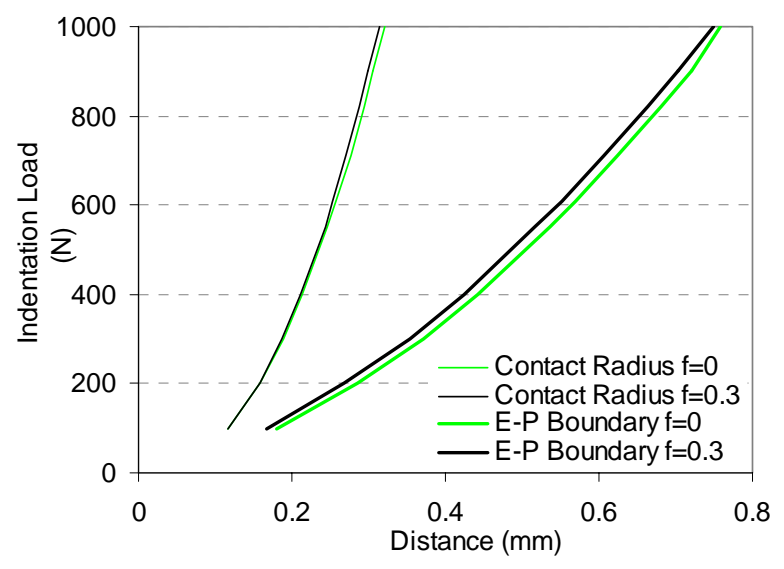

Figure 3.19 Friction effects on $P$-a and $P$-EPB relation

But the effects of friction are not so obvious. A friction coefficient 0.3 is used in the simulations. As shown in Figure 3.18 and Figure 3.19, it is found that friction affects both the surface deformation and material yielding. It clearly shows that residual deformation is decreased near the contact region with increased friction ( $f$ from 0 to 0.3 ). But beyond certain points, the residual deformation fields are increased due to increase of friction. Also with increased friction, the surface residual stress near the contact boundary decreases and EPB moves inwards. As shown in Figure 3.19, the friction coefficient has apparently effects on the elastic-plastic boundary, but the effect of contact radius is negligible. 


\section{CHAPTER 4}

\section{RESIDUAL DEFORMATION ANALYSES}

The residual surface deformation after spherical indentations is investigated on samples of IN783 superalloy. It served as one part of the effort to develop methods for extracting material mechanical properties using indentation technique. The residual in-plane $(u, v)$ and out-of-plane $(w)$ deformation are measured using a combined phase-shifting moiré and Twyman-Green interferometry. The elastic-plastic boundary is determined and material yield strength is obtained based on the measured out-of-plane deformation fringe patterns. The surface stress-strain is analyzed using the measured in-plane deformation. Further more, the measured contact radii and applied loads are used to establish post-yielding stress-strain data using Tabor's empirical relation.

\subsection{Specimen preparation}

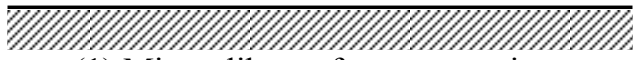

(1) Mirror-like surface preparation

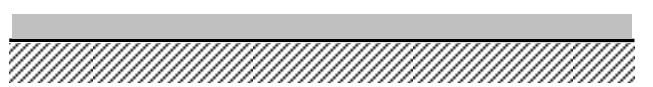

(2) Coating with photoresistive preparation

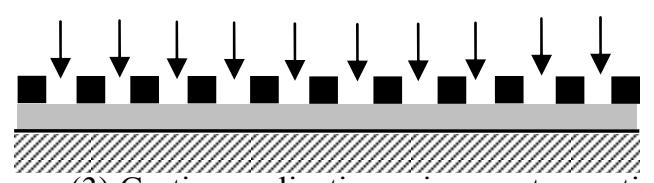

(3) Grating replication using master grating

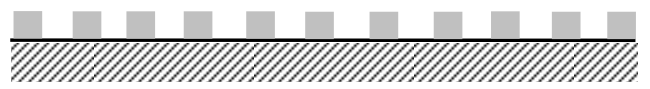

(4) Developing

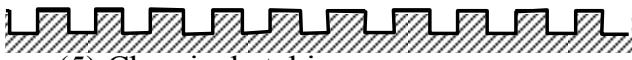

(5) Chemical etching

Figure 4.1 Making zero-thickness grating

A high quality surface finish is required for both moiré and Twyman-Green interferometry. It can be prepared using the standard polishing procedures. Sand papers are used for coarse grinding, and diamond suspension and alumina paste are used for the fine polishing down to $0.05 \mu \mathrm{m}$. The mirror-like surface finish is adequate for the out-of-plane deformation measurement using Twyman-Green interferometry, but for in-plane deformation measurement using moiré interferometry, a zero-thickness grating has to be made.

One way to make zero-thickness grating is through chemical etching technique. As shown in Figure 4.1, one thin layer of photoresist is applied on the specimen surface. After exposure and developing, a photoresist grating is now made on the specimen surface. Then the zero thickness grating is made through chemically etching the photoresist layer. Since the 
zero-thickness grating can reveal the real surface deformation, it is better than transferred grating.

A 600 lines/mm zero thickness grating is made on the IN783 superalloy specimens to study the residual in-plane and out-of-plane deformation. The stress-strain relation of this material can be simplified as elastic-linear plastic behavior as shown in Table $4.1^{[87]}$. If only out-of-plane residual deformation is desired, zero-thickness grating is not necessary. This can be used to quickly check the residual out-of-plane deformation, and may also be used to check the quality of surface condition.

Table 4.1 Mechanical properties of IN783 superalloy

\begin{tabular}{|l|l|}
\hline $\mathrm{E}=177.3 \mathrm{GPa}$ & $v=0.31$ \\
\hline$\sigma_{\mathrm{y}}=779 \mathrm{MPa}$ & $\sigma_{\mathrm{ult}}=1194 \mathrm{MPa}$ (ultimate strain 0.2) \\
\hline
\end{tabular}

Note: This material has undergone standard heat treatment: $1120^{\circ} \mathrm{C} / 1 \mathrm{hr} / \mathrm{AC}+845^{\circ} \mathrm{C} / 8 \mathrm{hrs} / \mathrm{AC}+720^{\circ} \mathrm{C} / 8 \mathrm{hrs} 50^{\circ} \mathrm{C} / \mathrm{hr} 620^{\circ} \mathrm{C} / 8 \mathrm{hrs} / \mathrm{AC}$

\subsection{Spherical indentation}

\section{Indentation system}

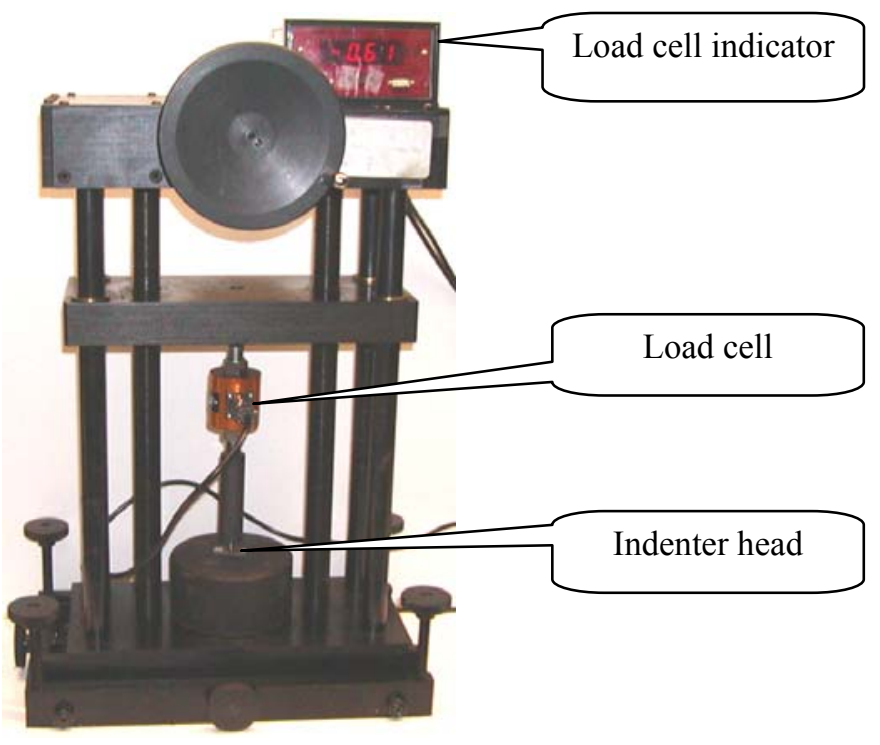

Figure 4.2 Indentation system for residual field measurements

As shown in Figure 4.2, a customized indentation system is built to conduct spherical indentation tests. It consists of a heavy duty loading frame and a $1.6 \mathrm{~mm}$ tungsten carbide ball indenter. The indenter head moves $30 \mu \mathrm{m}$ with one revolution of the wheel. A commercial Vickers indenter can also be replaced in this indentation system to conduct Vickers hardness tests. 


\section{Loading schedule}

A series of indentation tests are conducted with loads ranging from $100 \mathrm{~N}$ up to $1000 \mathrm{~N}$ as shown in Table 4.2. It is worth to note that for each case, the indenter stayed for 15 seconds and due to creep effects, the load may drop to a lower load.

The indent diameters are directly measured by an optical microscope (HIROX Hi-Scope). The indentation-induced residual deformation is measured using the following combined phase-shifting moiré and Twyman-Green interferometry.

Table 4.2 Indentation loading schedule

\begin{tabular}{|c|c|c|c|c|c|c|c|c|c|c|}
\hline Test \# & $\mathbf{1}$ & $\mathbf{2}$ & $\mathbf{3}$ & $\mathbf{4}$ & $\mathbf{5}$ & $\mathbf{6}$ & $\mathbf{7}$ & $\mathbf{8}$ & $\mathbf{9}$ & $\mathbf{1 0}$ \\
\hline Load(N) & 100 & 200 & 300 & 400 & 550 & 610 & 710 & 820 & 900 & 1000 \\
\hline
\end{tabular}

\subsection{Moiré and Twyman-Green interferometry}

\section{Optical principle}

Figure 4.3 and Figure 4.4 show the optical principle of the combined phase-shifting moiré and Twyman-Green interferometry for both in-plane $(u, v)$ and out-of-plane $(w)$ deformation measurement. The phase-shifting technique is introduced using a liquid crystal variable retarder (LCVR) to further improve the spatial resolution and automation. The advantage of this setup is that these two interferometers share the same imaging system and all the images have the same spatial resolution.

As shown in Figure 4.4, a HeNe laser beam with 45-degree linear polarization is split by a non-polarization beam splitter 1 (NBS 1). The reflected laser beam passes through a polarizer and the polarization direction is changed to $S$. The non-polarization beam splitter 2 (NBS 2) is served for both Twyman-Green interferometer and the imaging system. A $\lambda / 4$ waveplate is placed between the reference mirror and NBS 2 to change the polarization direction of the reference beam, so the laser beams entering imaging system from the reference mirror and specimen have mutually perpendicular polarization direction. It should be noted that the quarter wave plate as shown in Figure 4.3 is actually replaced by another LCVR, which is configured as shown in Figure 4.5 so that the fast or slow axis has 45-degree angle with the polarization direction of the incident beam. Then proper voltage is applied on the LCVR to make the reflected beam have the desired perpendicular polarization direction. Thus make it a controllable achromatic quarter waveplate.

The laser beam transmitted through NBS1 feeds the moire interferometer. A polarization beam splitter (PBS) splits the beam into two beams which have mutually perpendicular polarization direction. These two beams shine the specimen and finally go to the imaging system. As shown in Figure 4.4, phase-shifting is introduced in the imaging system as the two mutually perpendicular beams entering the LCVR, which is configured so that the $\mathrm{P}$ and $\mathrm{S}$ polarization directions are aligned to fast and slow axis, respectively. Through the LCVR 
digital interface controller, the phase-shifting for both moiré interferometry and Twyman-Green interferometry is controlled by a PC. A polarizer is placed after the LCVR to view the fringe pattern in the imaging system ${ }^{[83]}$.

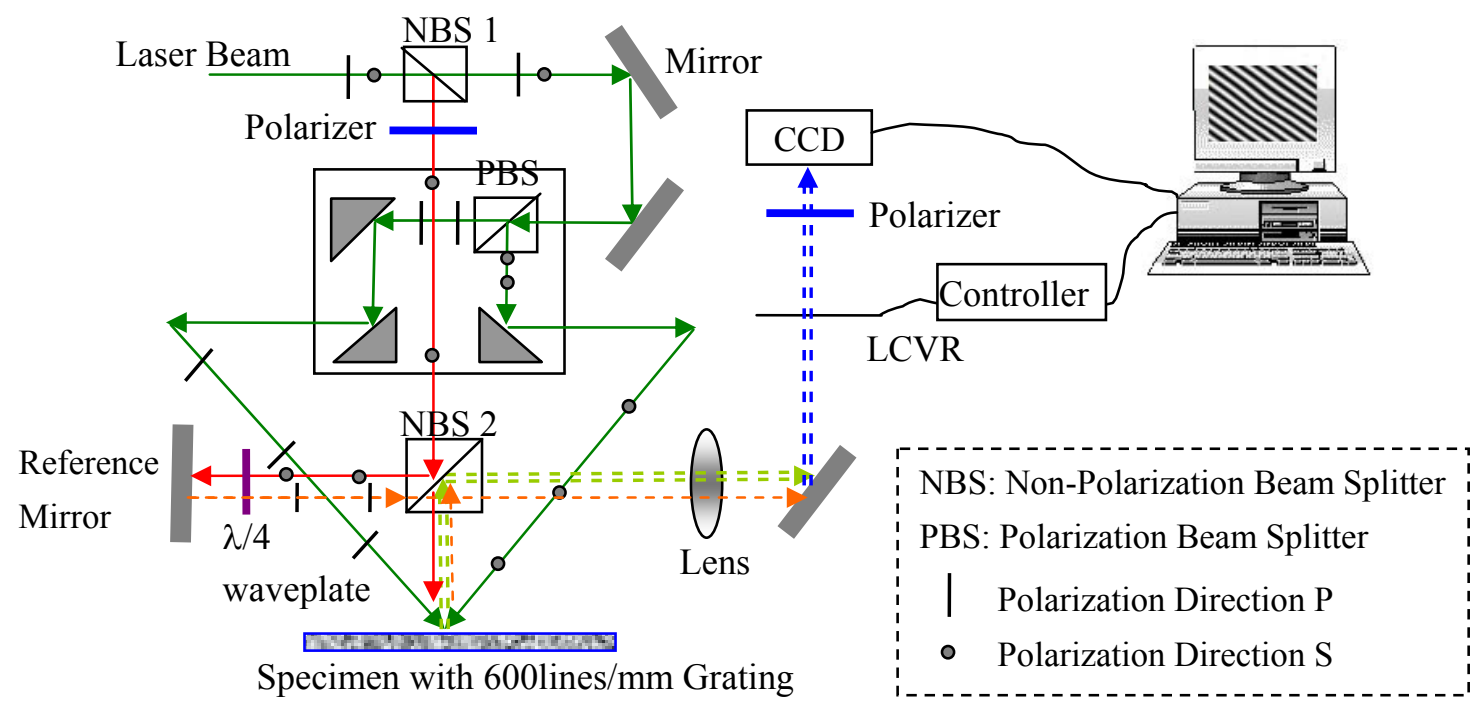

Figure 4.3 Combined phase-shifting moiré and Twyman-Green Interferometry

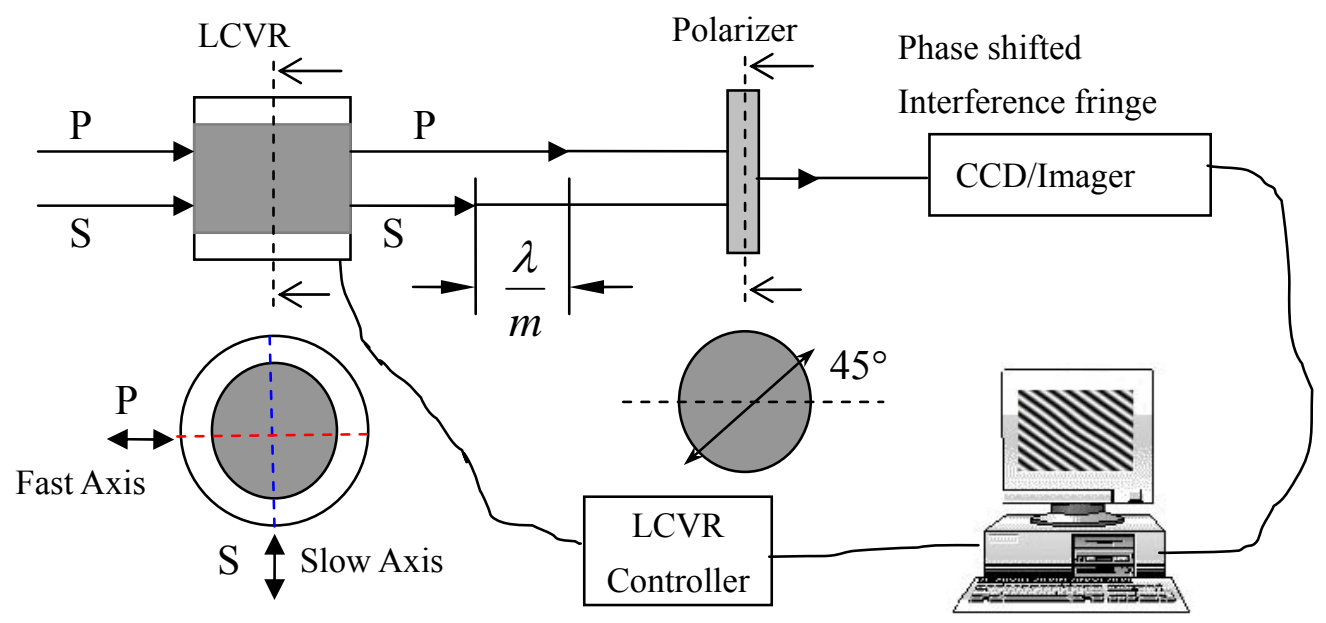

Figure 4.4 Configuration of LCVR for combined optical setup
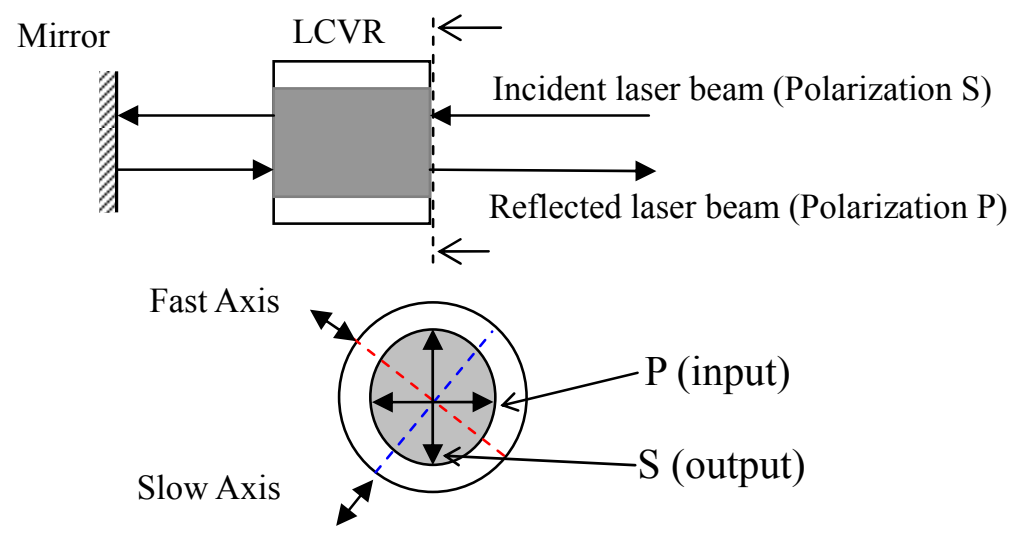
Figure 4.5 Use LCVR as an achromatic quarter waveplate

\section{Laboratory setup}

Figure 4.6 shows the laboratory setup. A specimen with 600Lines/mm zero thickness grating is placed on a 6-Degree of Freedom (DOF) stage. A ring white light source between NBS 2 and the specimen is used for fine focusing and indentation diameter measurement. Meadowlark Optics ${ }^{\mathrm{TM}}$ D2040 digital interface and National Instruments ${ }^{\mathrm{TM}}$ frame grabber (PCI-1408) are used for the phase-shifting control and image acquisition using customized software. The sensitivity of current system is $0.8334 \mu \mathrm{m} /$ fringe for in-plane $(u, v)$ deformation and $0.3164 \mu \mathrm{m} /$ fringe for out-of-plane $(w)$ deformation using a $\mathrm{HeNe}$ laser (wavelength $\lambda=0.6328 \mu \mathrm{m})$.

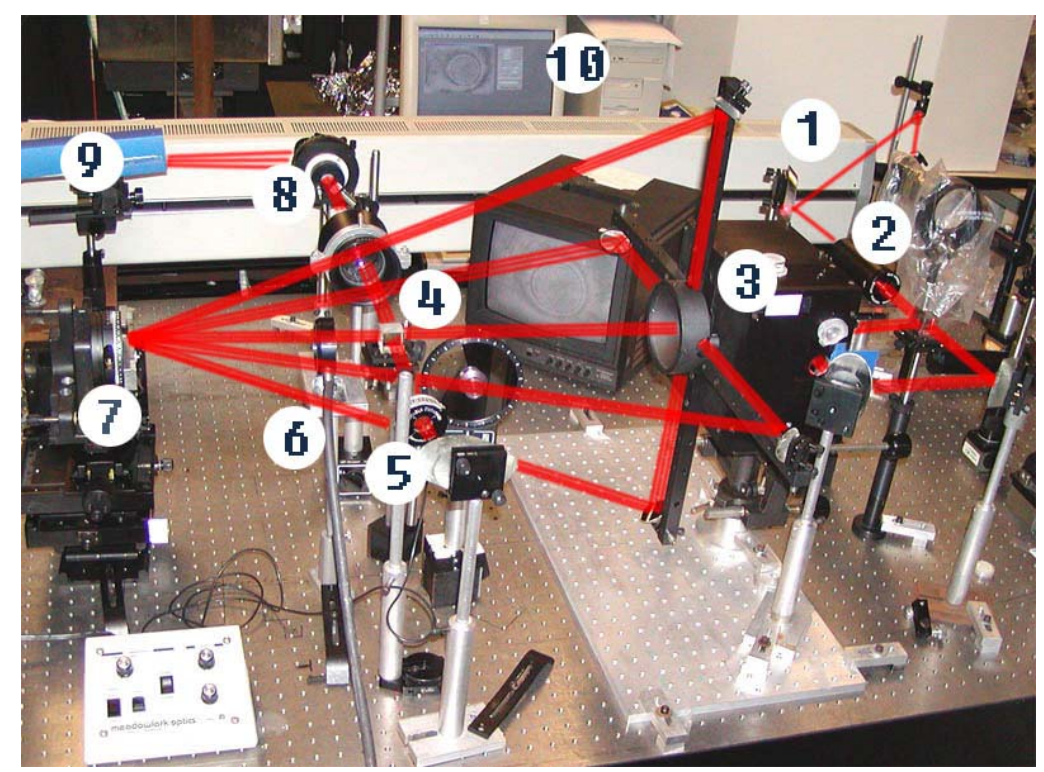

(1) HeNe Laser (2) beam expander (3) moiré interferometry unit (4) NBS2, Beam splitter

(5) $\lambda / 4$ wave plate $\&$ reference mirror (6) ring light source (7) specimen holder with 6-DOF adjustment (8) LCVR and polarizer (9) CCD camera on adjustable stage (10) PC with image acquisition and LCVR control

Figure 4.6 Laboratory setup

\subsection{Image acquisition, processing and analysis}

The software controls the image acquisition and phase-shifting is separate for each application. It is inconvenient to conduct the phase-shifting interferometric test. Customized software which can acquire images while sending phase-shifting signals is developed $(\mathrm{C} / \mathrm{C}++)$. A screen snap shot is shown in Figure 4.7. It is based on the National Instruments ${ }^{\text {TM }}$ frame grabber (PCI-1408), image acquisition library IMAQ ${ }^{\mathrm{TM}}$, and a shareware development kit (SDK) NTPort ${ }^{\mathrm{TM}[85]}$. The shareware is used to access computer hardware to directly control the Meadowlark Optics ${ }^{\mathrm{TM}}$ D2040 digital interface for LCVR.

The software is programmed by calling IMAQ ${ }^{\mathrm{TM}}$ low level functions for image acquisition and accessing LCVR controller using NTPort ${ }^{\mathrm{TM}}$ SDK functions. It is implemented by using 
dynamic link library (DLL) and applying multi-threading technique. Using this software, the LCVR and image acquisition are controlled simultaneously using pre-defined parameters, which is critical for this phase-shifting application. It displays the fringe patterns in real-time. Specially, with one button click, it automatically captures 4 phase-shifted fringe images and shows the phase results immediately. Before each image acquisition, it automatically sends the voltage to LCVR controller using pre-defined parameters and waits for a pre-defined time delay. With this automatic control, the phase-shifting and image acquisition for one measurement can be done without any human-related delays. Since vibration is always of great concern in most laser interferometric applications, this procedure can greatly reduce the human error and capture consistent images. Besides, the automatic phase map calculation and display let the operator immediately check the quality of current phase-shifting image acquisition. If satisfied, one click can save all the results to a set of pre-defined file names.

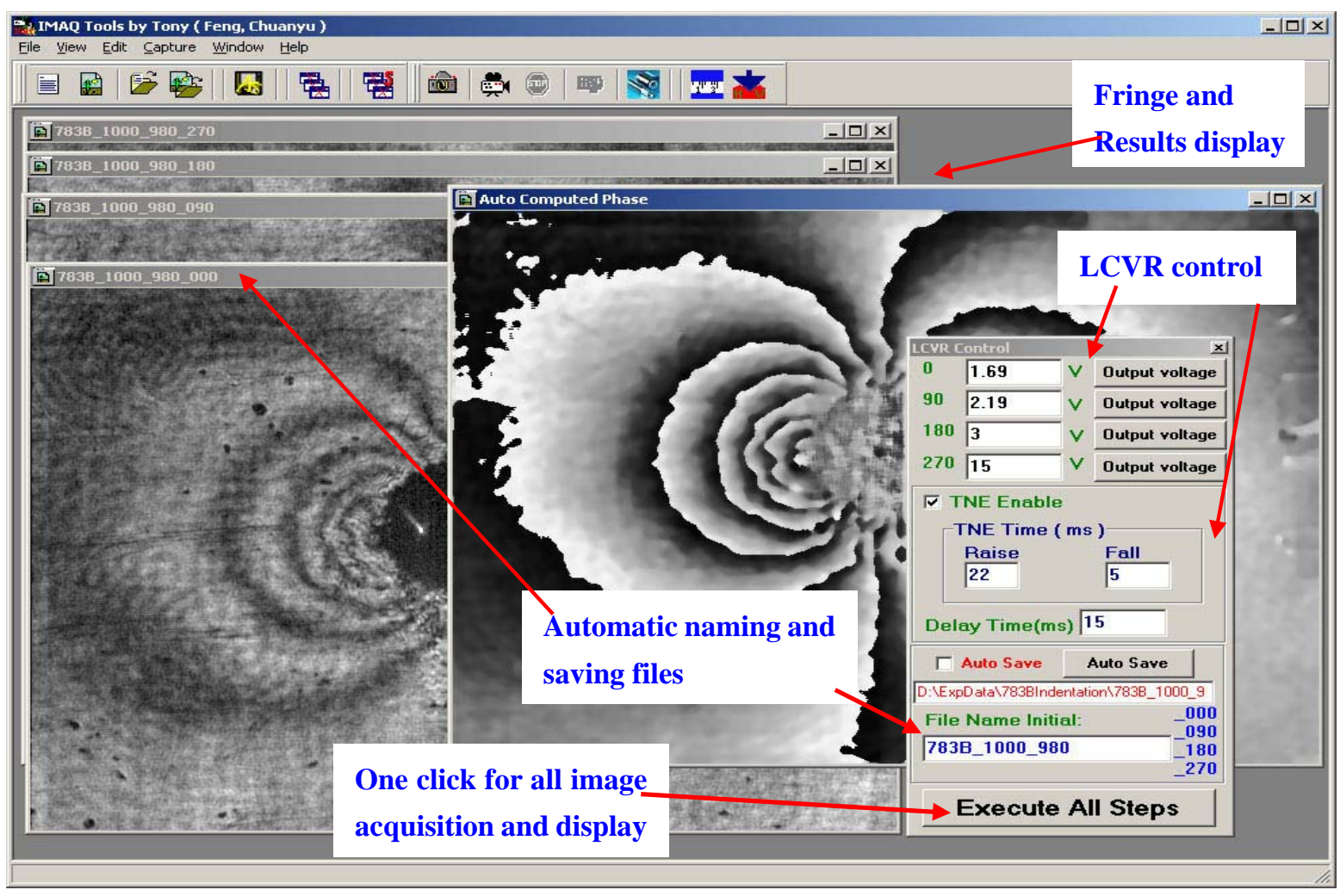

Figure 4.7 IMAQTools software for image acquisition and phase-shifting control

\subsection{Results and discussions}

\section{Residual deformation fields}

Figure 4.8 to Figure 4.17 show the residual deformation fields and corresponding phase maps for all 10 sets of IN783 indentation tests. The size of the covered region of each image is $4.18 \mathrm{~mm} \times 3.14 \mathrm{~mm}$. Each fringe represents $0.8333 \mu \mathrm{m}$ displacement in $u, v$ fields and $0.3164 \mu \mathrm{m}$ 
displacement in $w$ field. It is shown that in-plane deformation field $u$ and $v$ are basically the same and out-of-plane deformation field $w$ is circular shape.

It is noticed that the outer fringe patterns in $w$ field are symmetric, which indicate that the residual stress before indentation tests is negligible ${ }^{[22]}$. At and near the contact boundary, it shows irregular fringes for both in-plane and out-of-plane deformation fields. That is an indication of the material yielding. Underwood ${ }^{[22]}$ concluded this type of non-symmetric fringe to the instability of the yielding point and Lüder's strain characteristic. It is also observed that the region of irregular deformation is easier to identify in $w$ field. This is due to the higher sensitivity in $w$ field than $u, v$ fields under current setup. At and near the indented zone, due to damage of the zero thickness grating and large curvature, $u, v$ and $w$ fields are not obtainable.

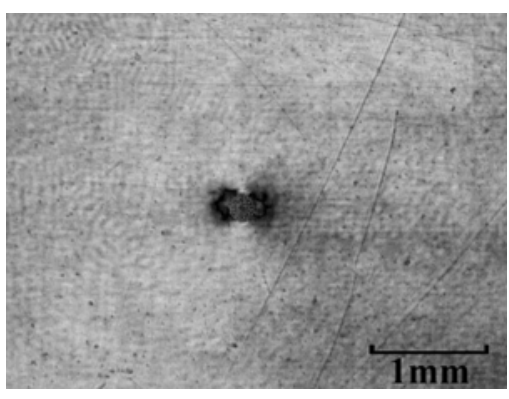

$u$ field

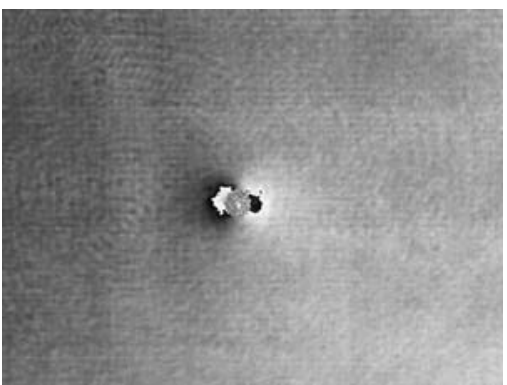

$u$ field phase map

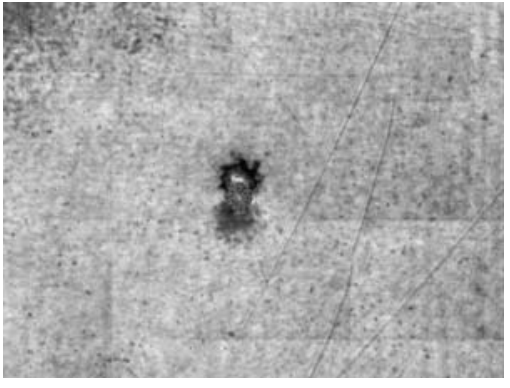

$v$ field

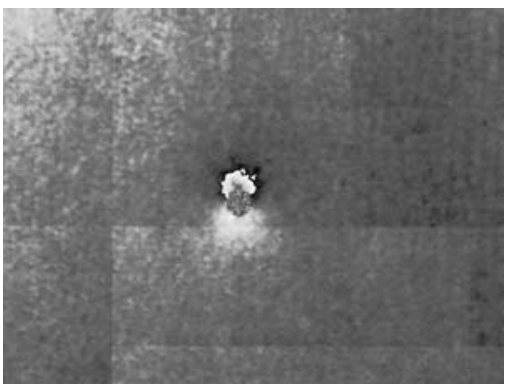

$v$ field phase map

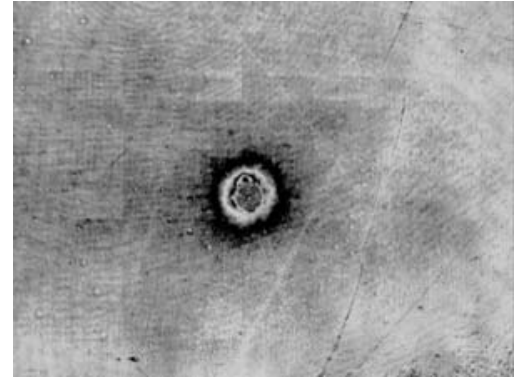

$w$ field

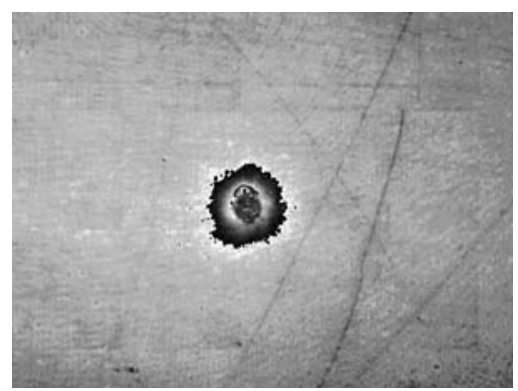

$w$ field phase map

Figure 4.8 Residual deformation after $100 \mathrm{~N}$ indentation 


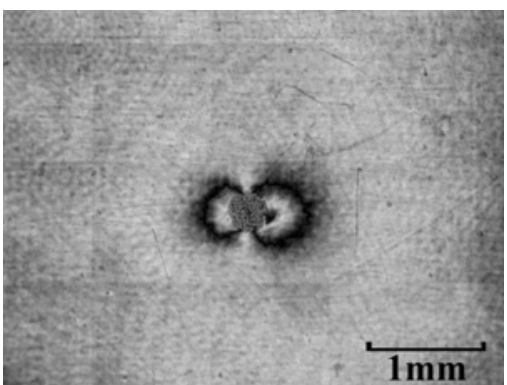

$u$ field

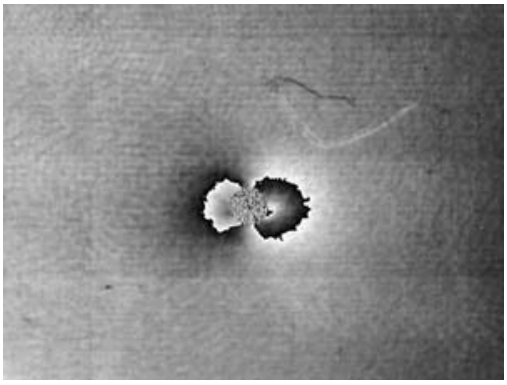

$u$ field phase map

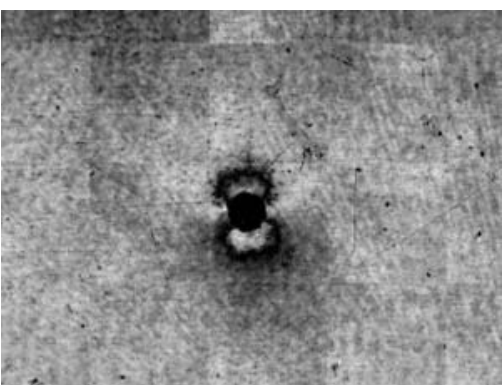

$v$ field

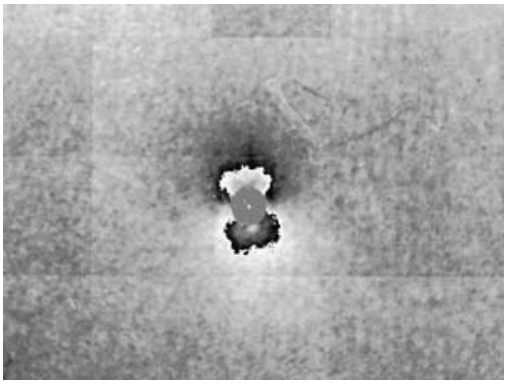

$v$ field phase map

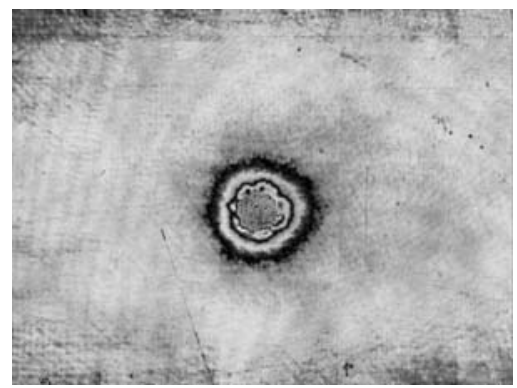

$w$ field

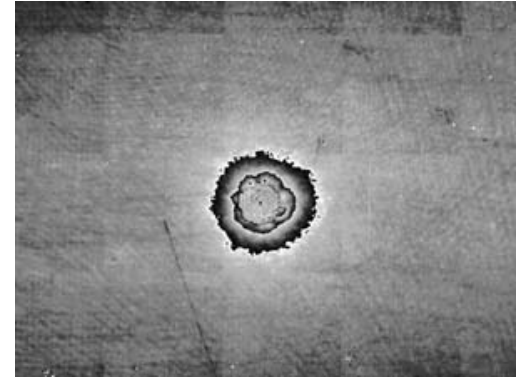

$w$ field phase map

Figure 4.9 Residual deformation after $200 \mathrm{~N}$ indentation

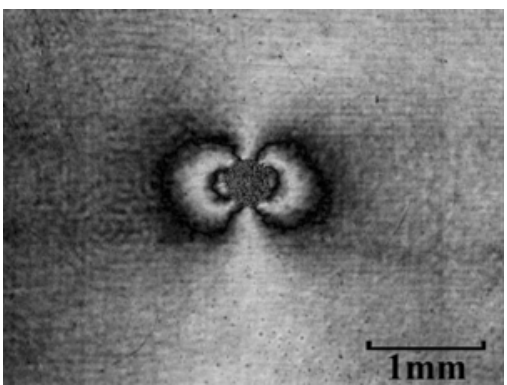

$u$ field

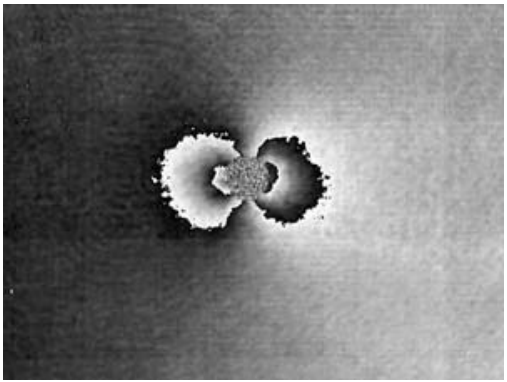

$u$ field phase map

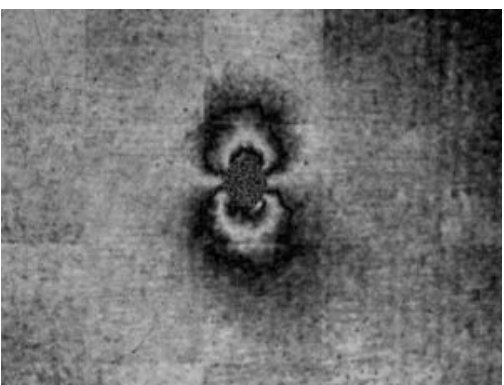

$v$ field

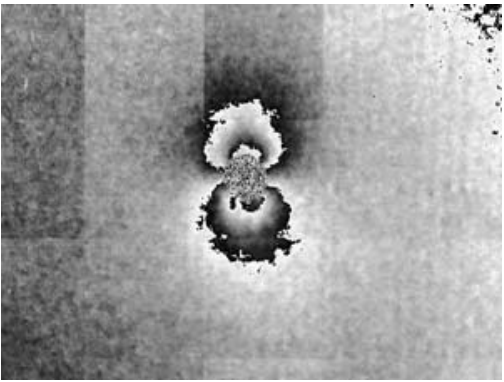

$v$ field phase map

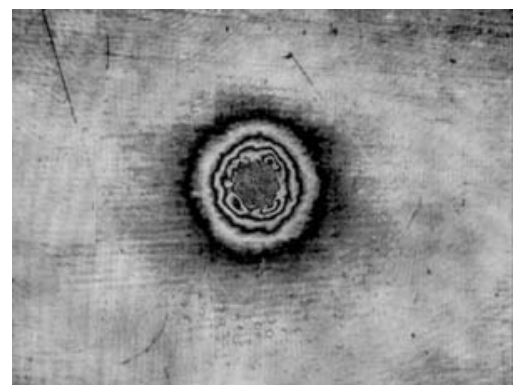

$w$ field

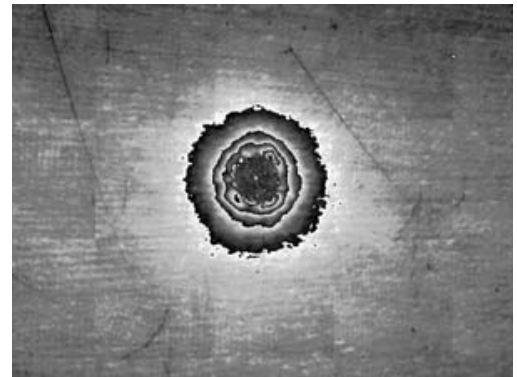

$w$ field phase map

Figure 4.10 Residual deformation after $300 \mathrm{~N}$ indentation 


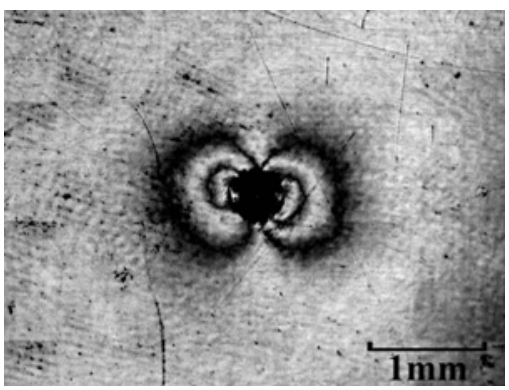

$u$ field

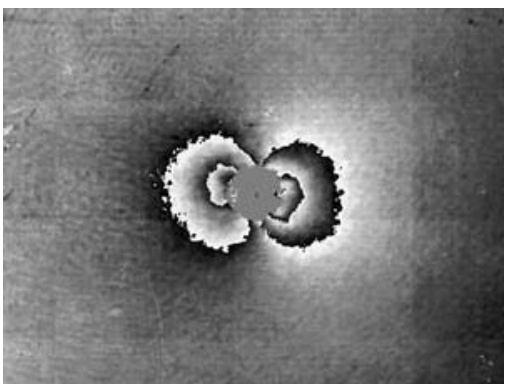

$u$ field phase map

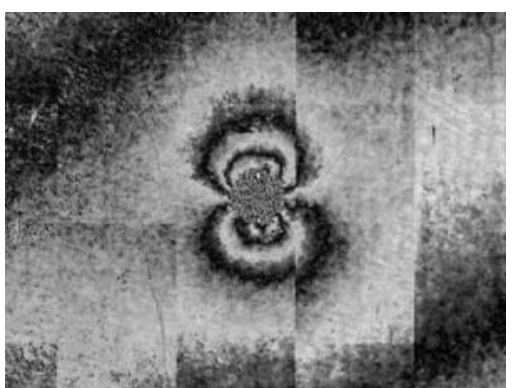

$v$ field

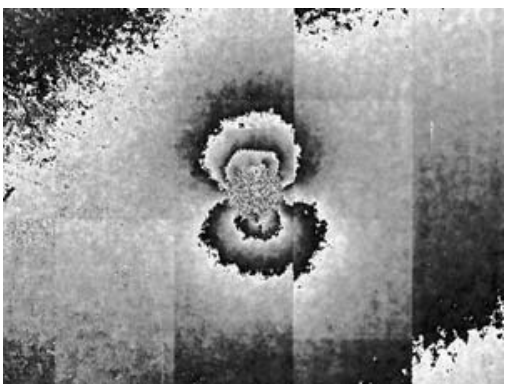

$v$ field phase map

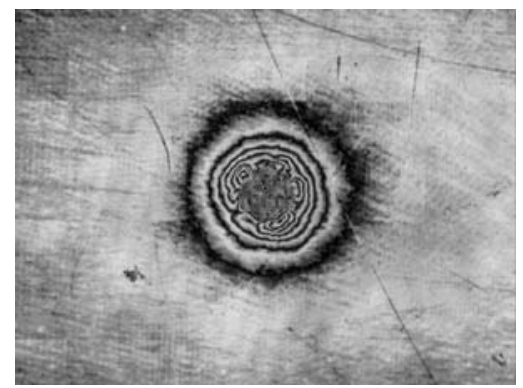

$w$ field

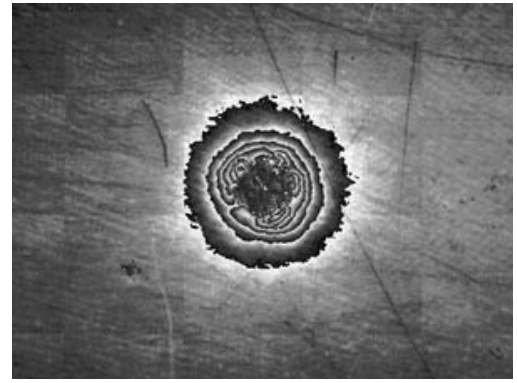

$w$ field phase map

Figure 4.11 Residual deformation after $400 \mathrm{~N}$ indentation

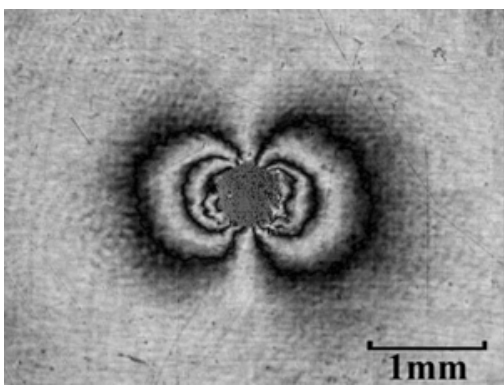

$u$ field

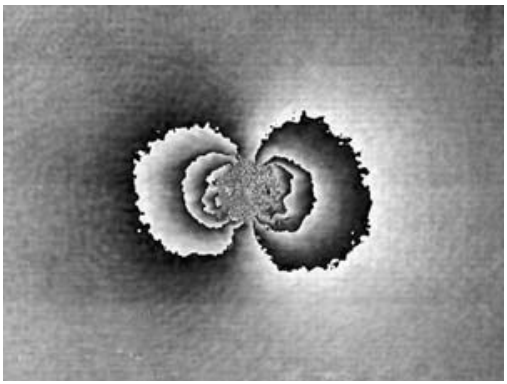

$u$ field phase map

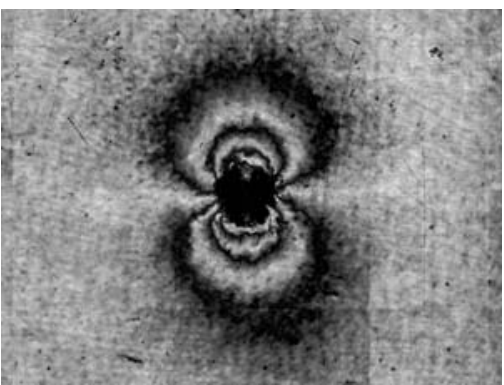

$v$ field

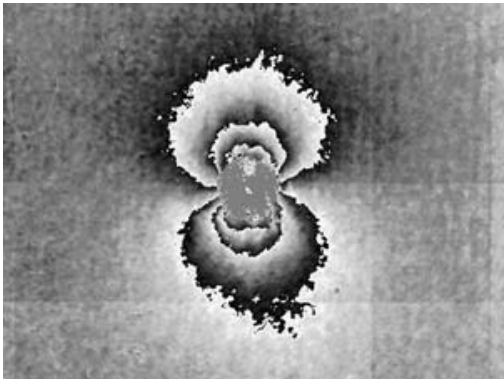

$v$ field phase map

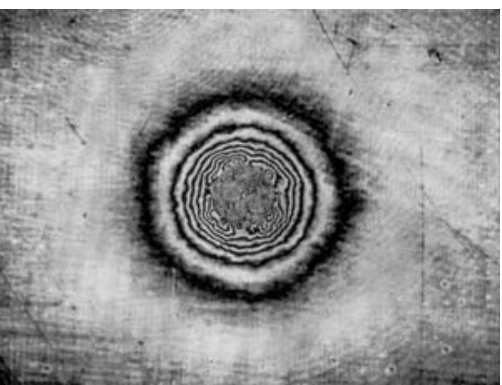

$w$ field

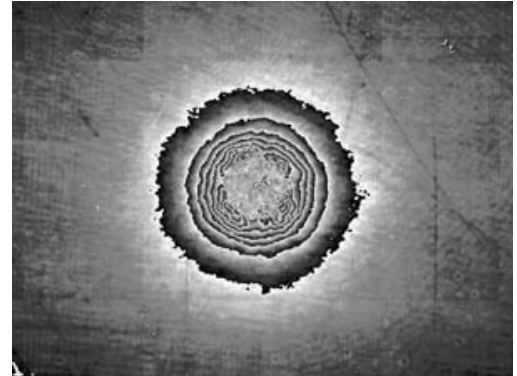

$w$ field phase map

Figure 4.12 Residual deformation after 550N indentation 


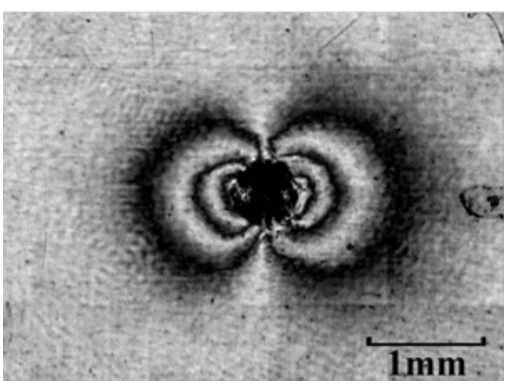

$u$ field

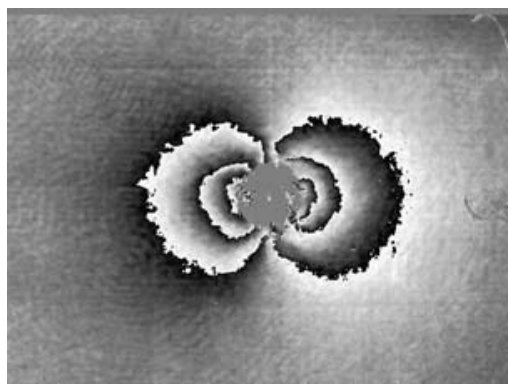

$u$ field phase map

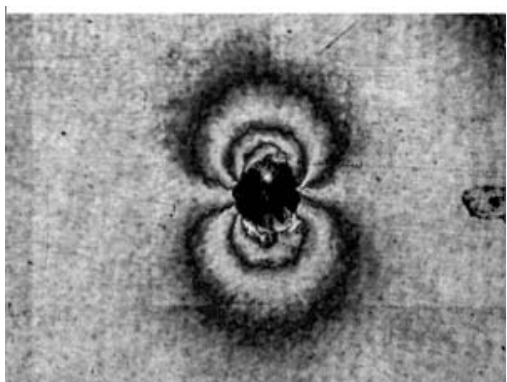

$v$ field

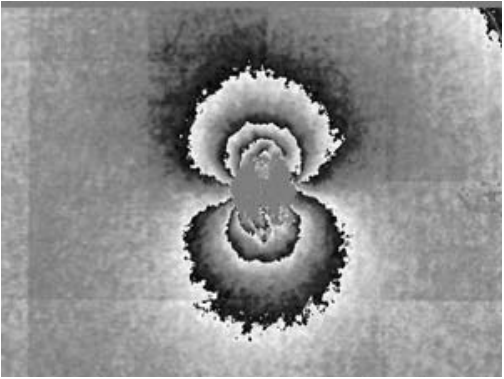

$v$ field phase map

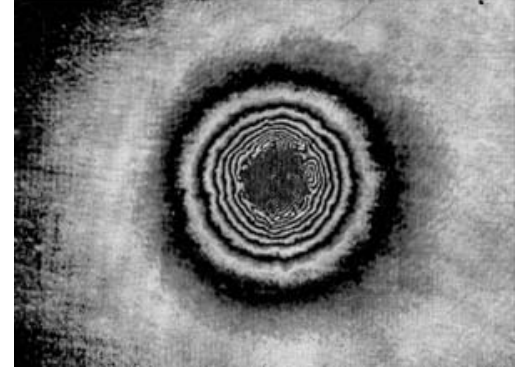

$w$ field

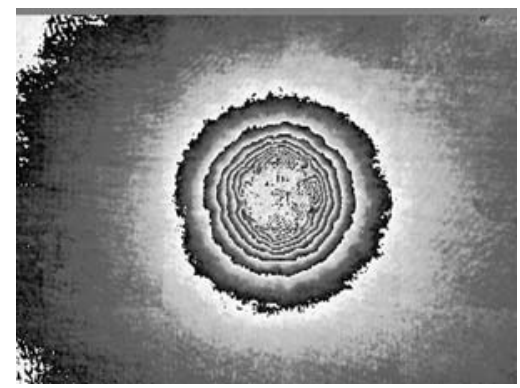

$w$ field phase map

Figure 4.13 Residual deformation after $610 \mathrm{~N}$ indentation

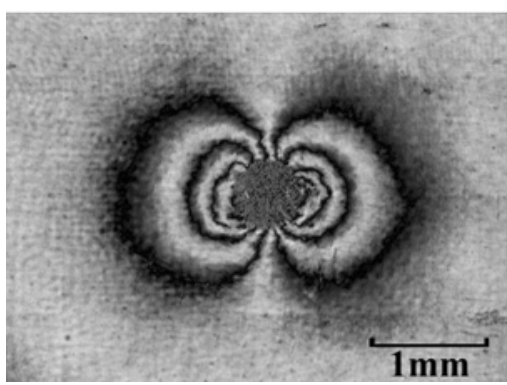

$u$ field

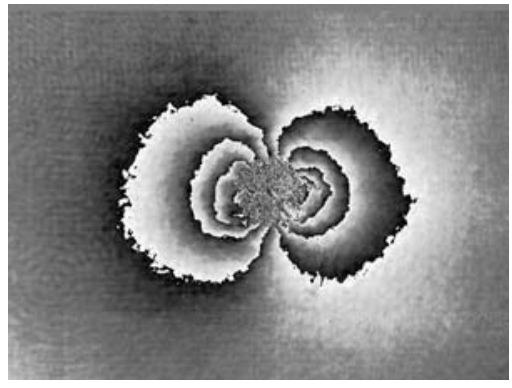

$u$ field phase map

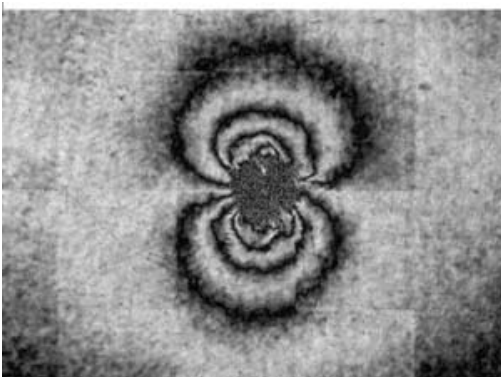

$v$ field

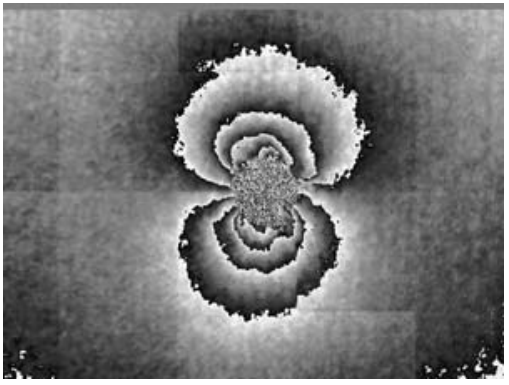

$v$ field phase map

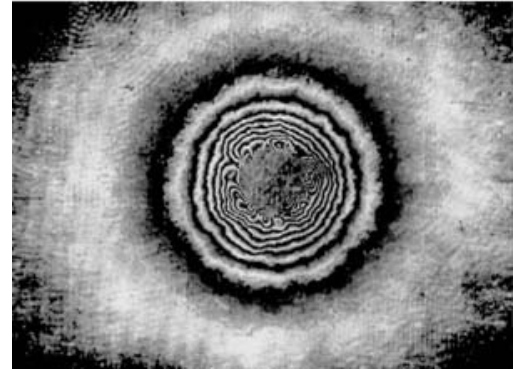

$w$ field

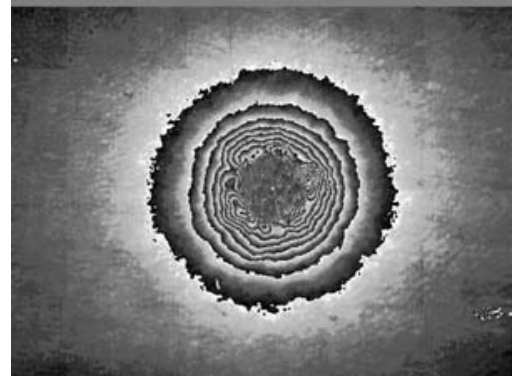

$w$ field phase map

Figure 4.14 Residual deformation after 710N indentation 


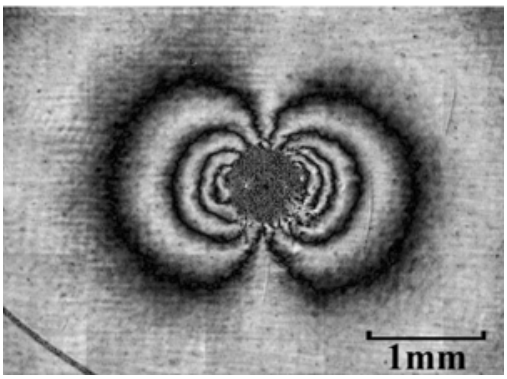

$u$ field

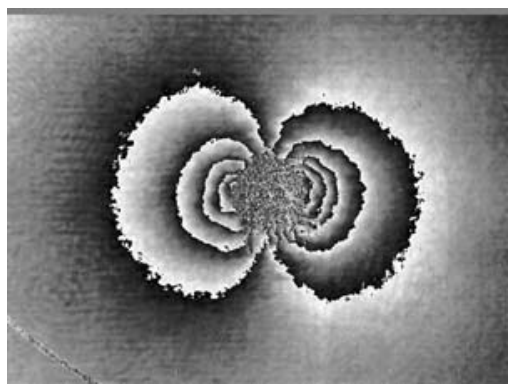

$u$ field phase map

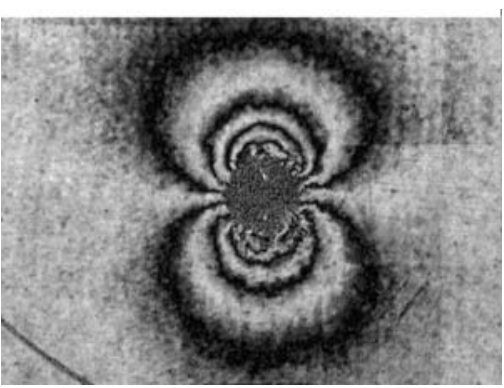

$v$ field

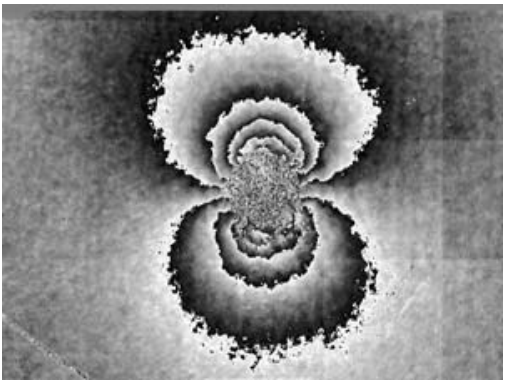

$v$ field phase map

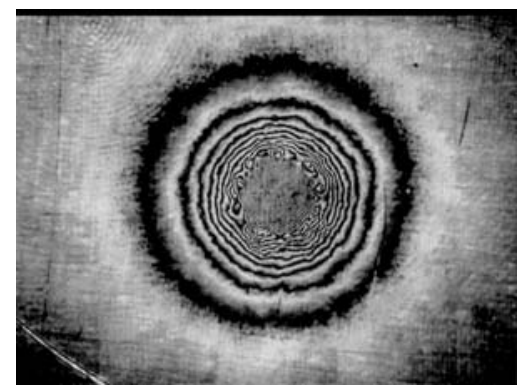

$w$ field

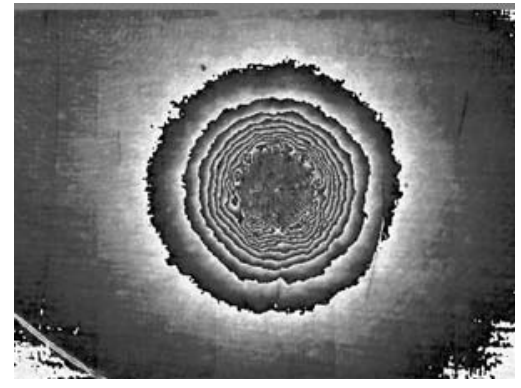

$w$ field phase map

Figure 4.15 Residual deformation after 820N indentation

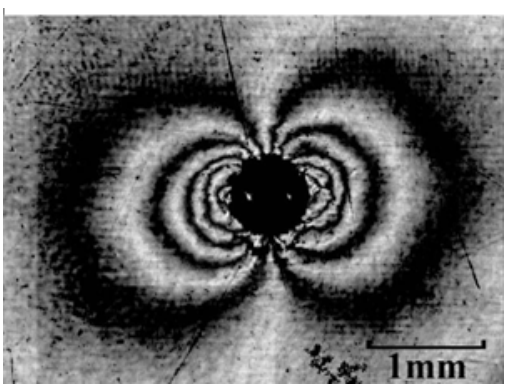

$u$ field

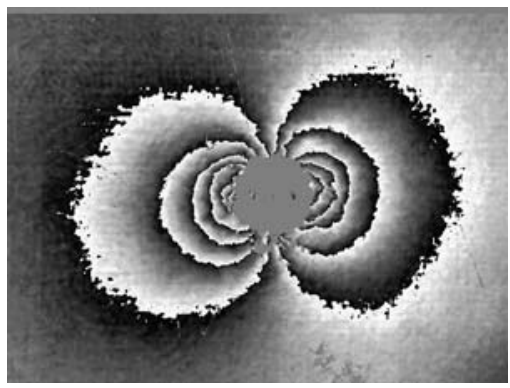

$u$ field phase map

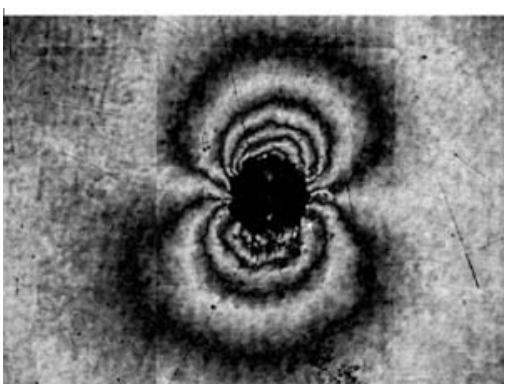

$v$ field

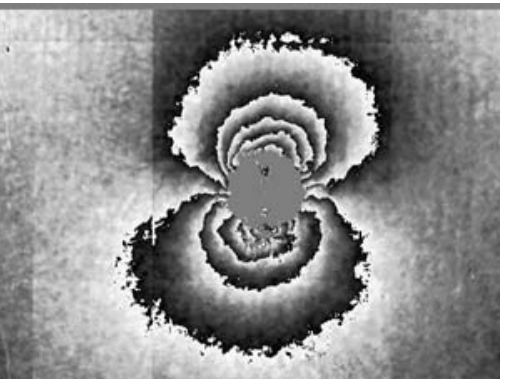

$v$ field phase map

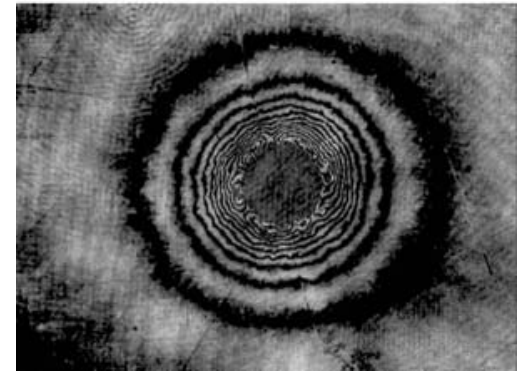

$w$ field

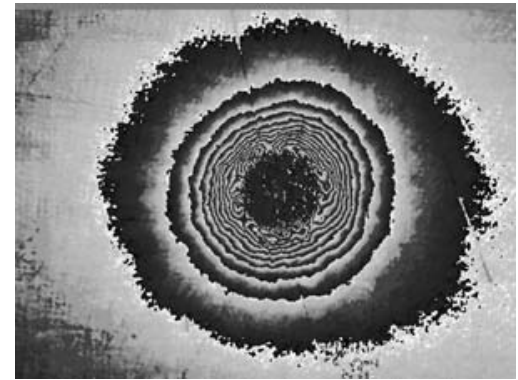

$w$ field phase map

Figure 4.16 Residual deformation after 900N indentation 


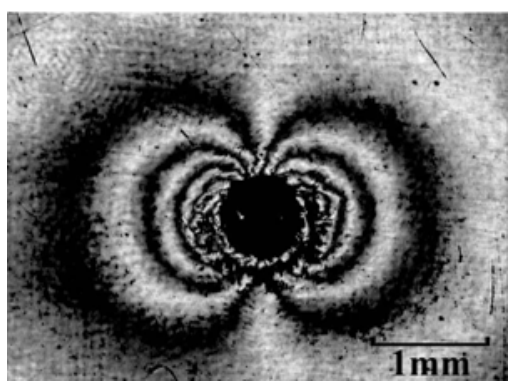

$u$ field

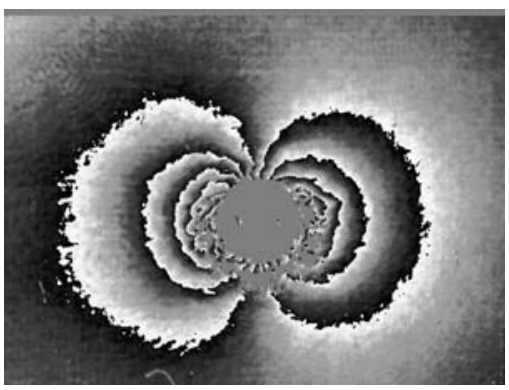

$u$ field phase map

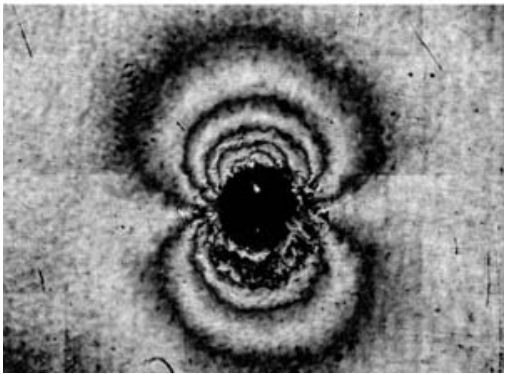

$v$ field

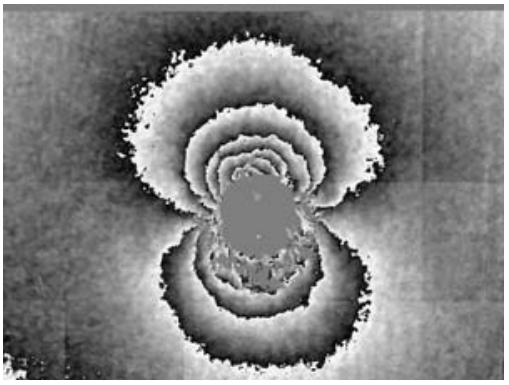

$v$ field phase map

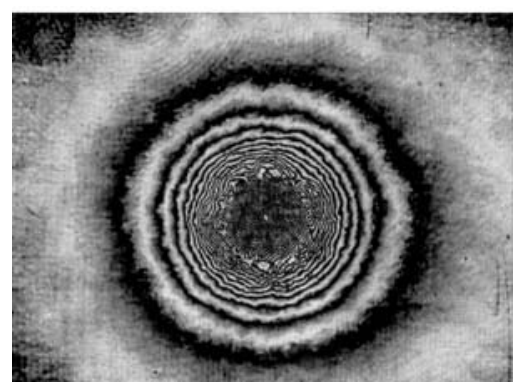

$w$ field

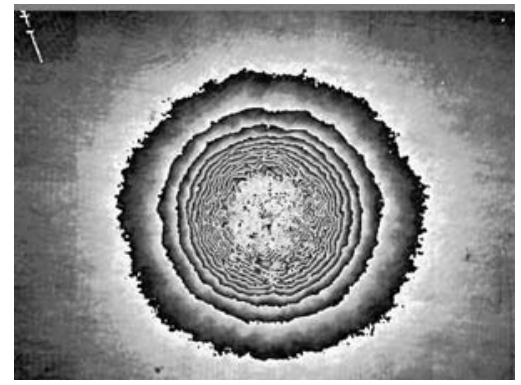

$w$ field phase map

Figure 4.17 Residual deformation after $1000 \mathrm{~N}$ indentation

\section{Residual deformation analyses}

The residual out-of-plane $(w)$ deformation has distinct feature. It is found that all irregular fringe patterns in $w$ fields start from the third black fringe under current configuration except for $100 \mathrm{~N}$ and $200 \mathrm{~N}$ indentation, in which the residual deformation is too small to view it clearly. This irregularity may be used to assess the material yielding characteristics, i.e., the degree of ductility or brittleness.

The center of the third black fringe is marked for each test. It will be proved later that these are the elastic-plastic boundary (EPB) on the specimen surface. For small loading condition, the third black fringe is not available, so the point where fringe becomes irregularly is chosen as the elastic-plastic boundary. Figure 4.18 to Figure 4.21 show the residual fields marked with these identified elastic-plastic boundaries.

The plots of loads vs. contact radii and load vs. elastic-plastic boundaries are shown in Figure 4.22. The loads and contact radii follow a power law as stated in Meyer's law. The curve fitting also shows that the loads and elastic-plastic boundaries also follow a power law relation same as predicted by finite element simulations. 


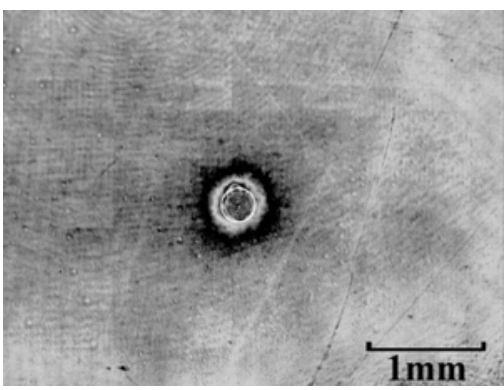

EPB in $w$ field after $100 \mathrm{~N}$

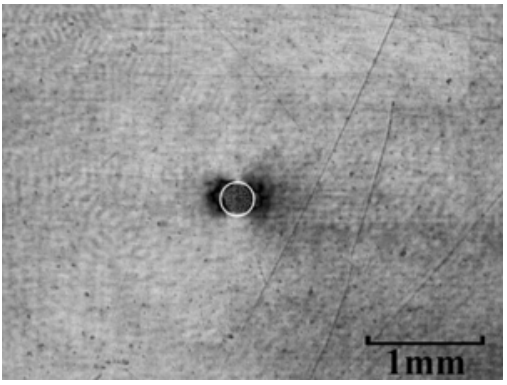

EPB in $u$ field after $100 \mathrm{~N}$

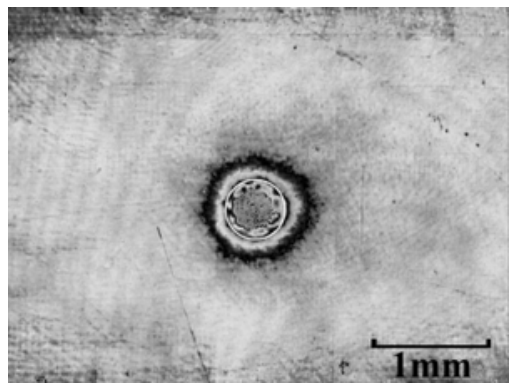

EPB in $w$ field after $200 \mathrm{~N}$

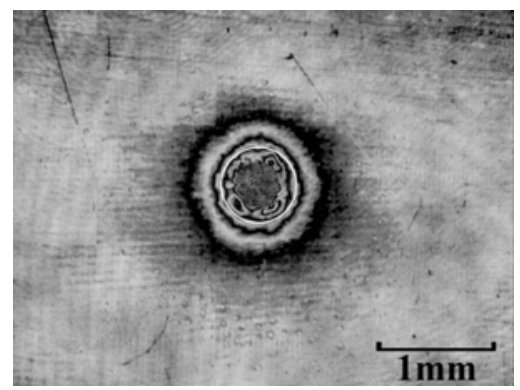

EPB in $w$ field after $300 \mathrm{~N}$

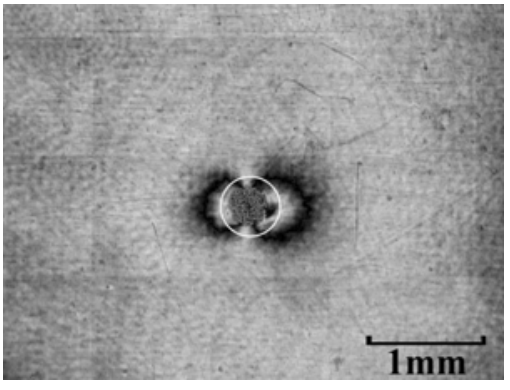

EPB in $u$ field after $200 \mathrm{~N}$

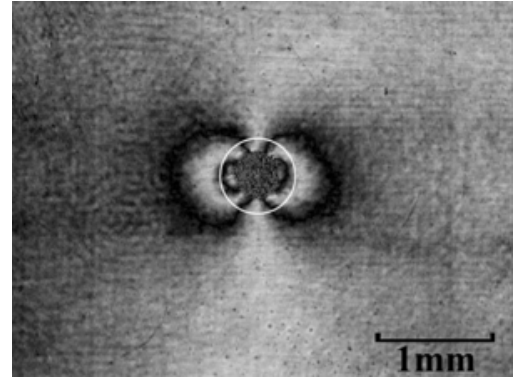

EPB in $u$ field after $300 \mathrm{~N}$

Figure 4.18 Identified elastic-plastic boundary (EPB) (1)

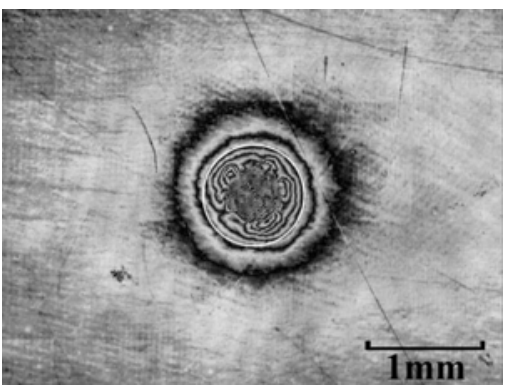

EPB in $w$ field after $400 \mathrm{~N}$

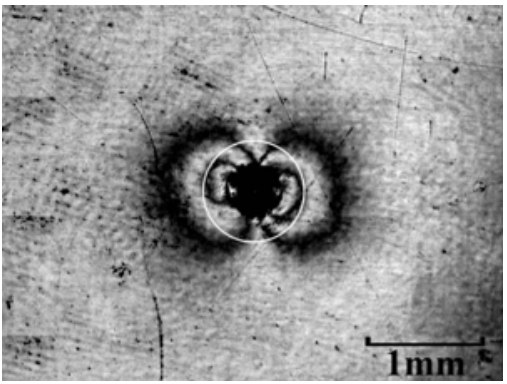

EPB in $u$ field after $400 \mathrm{~N}$

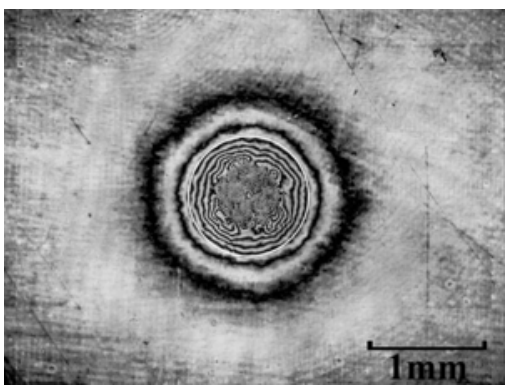

EPB in $w$ field after $550 \mathrm{~N}$

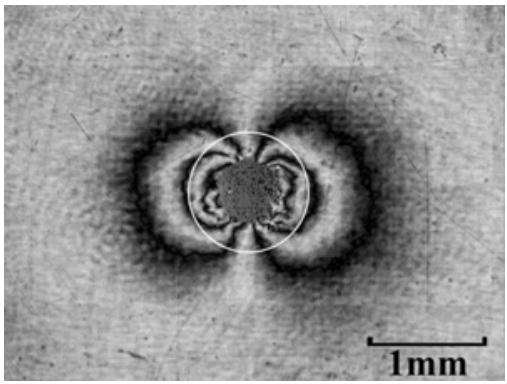

EPB in $u$ field after $550 \mathrm{~N}$

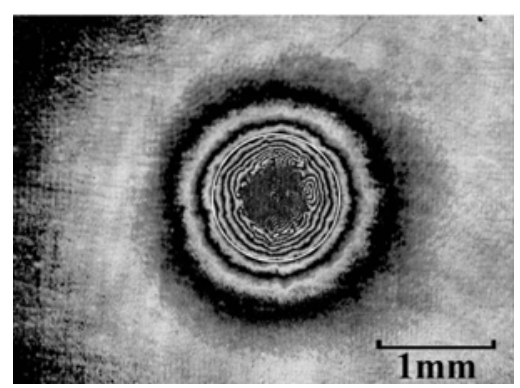

EPB in $w$ field after $610 \mathrm{~N}$

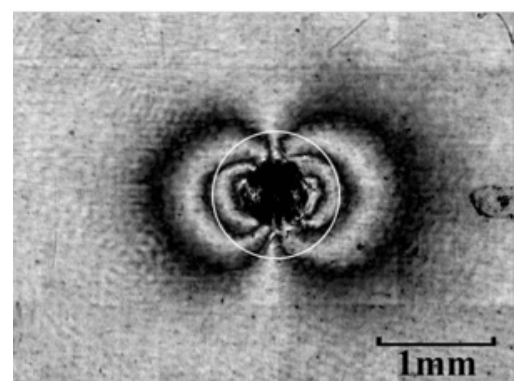

EPB in $u$ field after $610 \mathrm{~N}$

Figure 4.19 Identified elastic-plastic boundary (2) 


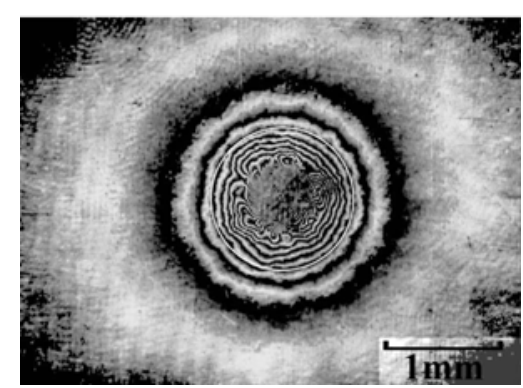

EPB in $w$ field after $710 \mathrm{~N}$

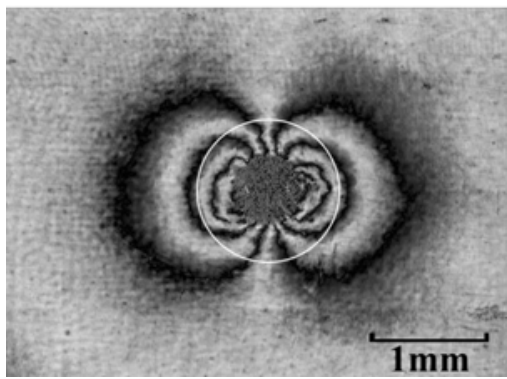

EPB in $u$ field after $710 \mathrm{~N}$

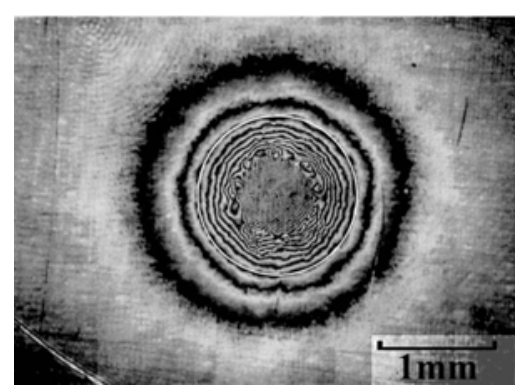

EPB in $w$ field after $820 \mathrm{~N}$

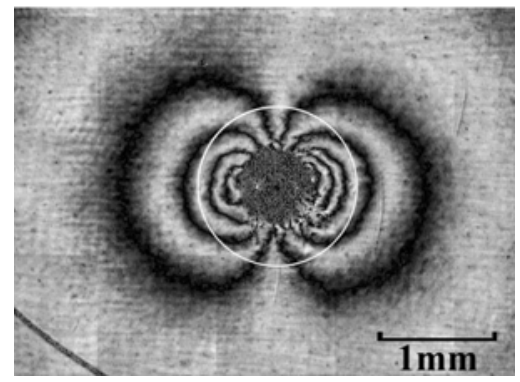

EPB in $u$ field after $820 \mathrm{~N}$

Figure 4.20 Identified elastic-plastic boundary (3)

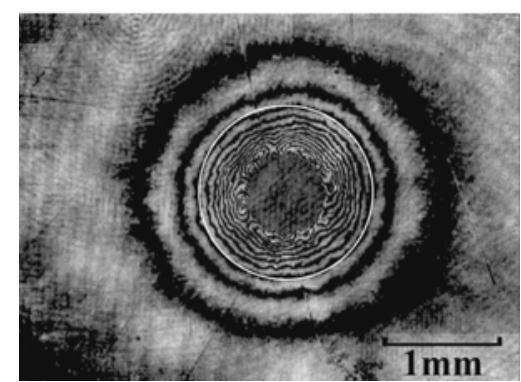

EPB in $w$ field after $900 \mathrm{~N}$

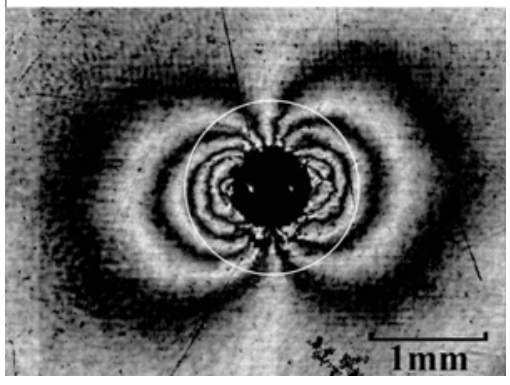

EPB in $u$ field after $900 \mathrm{~N}$

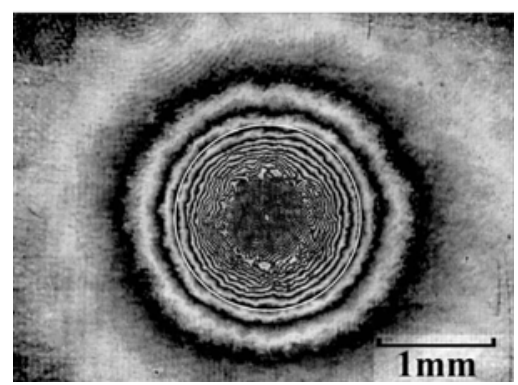

EPB in $w$ field after $1000 \mathrm{~N}$

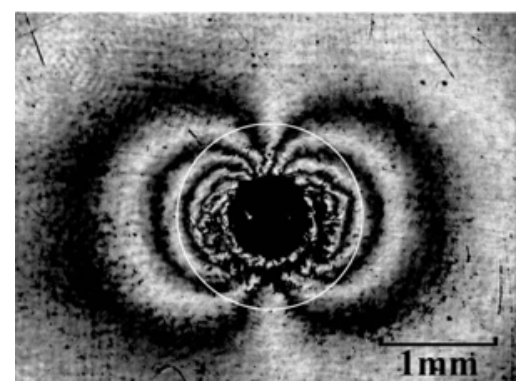

EPB in $u$ field after $1000 \mathrm{~N}$

Figure 4.21 Identified elastic-plastic boundary (4) 


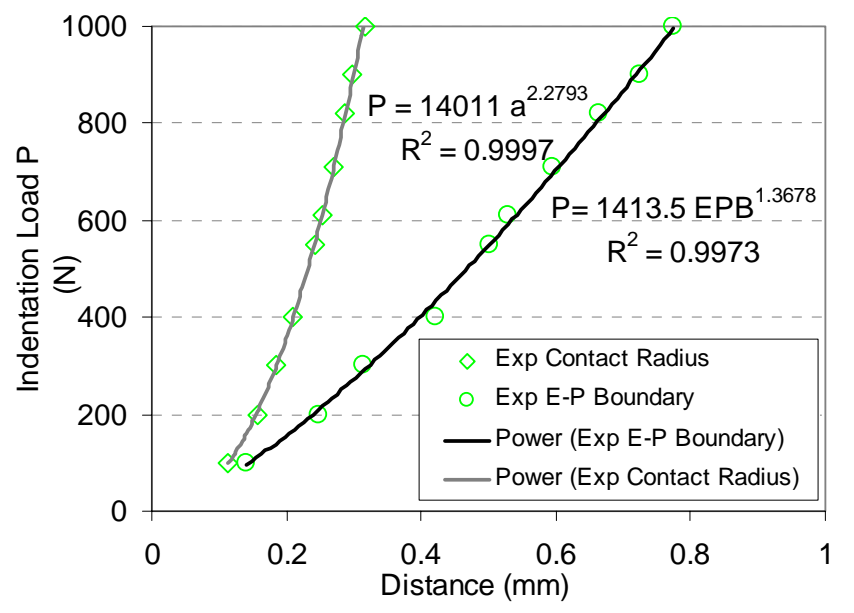

Figure 4.22 Experimental contact radii and elastic-plastic boundaries

In order to determine the yield strength, surface residual stress analysis is carried out as discussed in Chapter 3. The absolute residual in-plane displacements are determined first to derive the surface residual stress distribution.

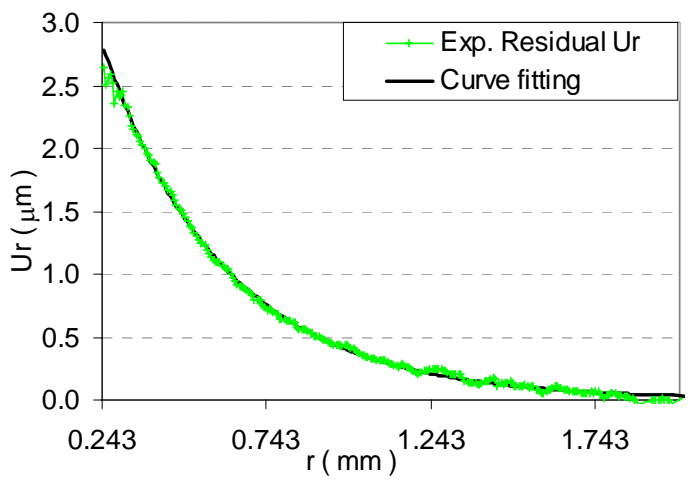

(a) Surface residual in-plane deformation

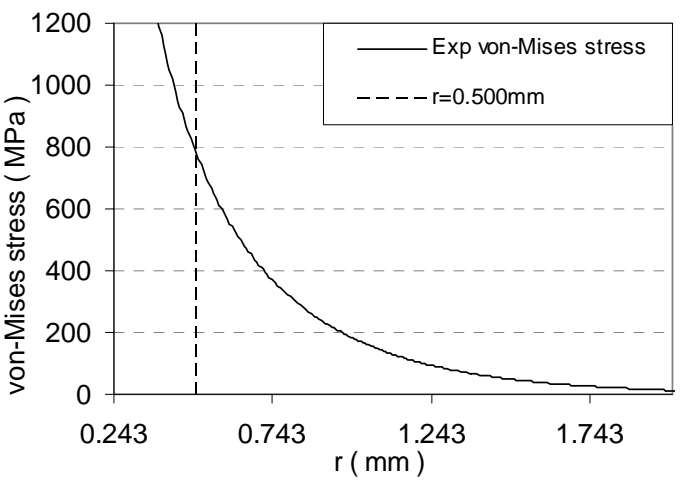

(b) Equivalent von-Mises stress distribution

Figure 4.23 Determination of yield strength after 550N indentation

The absolute in-plane displacements are determined using the symmetric outmost fringe in $u$ or $v$ fields. Under current configuration, it is known that the symmetric outmost black fringes represent $1 / 2$ fringe of in-plane displacement $(0.4167 \mu \mathrm{m})$ for $u$ or $v$ fields in Figure 4.8 to Figure 4.17. Based on this reference, the $u$ or $v$ displacement fields are converted into absolute displacements through a series of phase map unwrapping and linear transformations.

The typical absolute in-plane displacement distribution is shown in Figure 4.23(a). To get smooth strain and stress distribution, the in-plane displacement must be smoothed first, such as curve fitting. Then the surface residual von-Mises stress can be calculated following procedures described in \$3.4 of Chapter 3. The typical result is shown in Figure 4.23(b). The stress at identified elastic-plastic boundary is then obtained as the measured yield strength. The results from all the residual deformation analyses are shown in Figure 4.24. The primary ordinate (vertical) axis shows the yield strength, and the secondary ordinate axis shows the 
corresponding error for each loading case. Maximum error is $-6.1 \%$ and the average is only $-1.2 \%$ lower than the known yield strength. The results confirmed that the identified elastic-plastic boundaries are the real elastic-plastic boundaries.

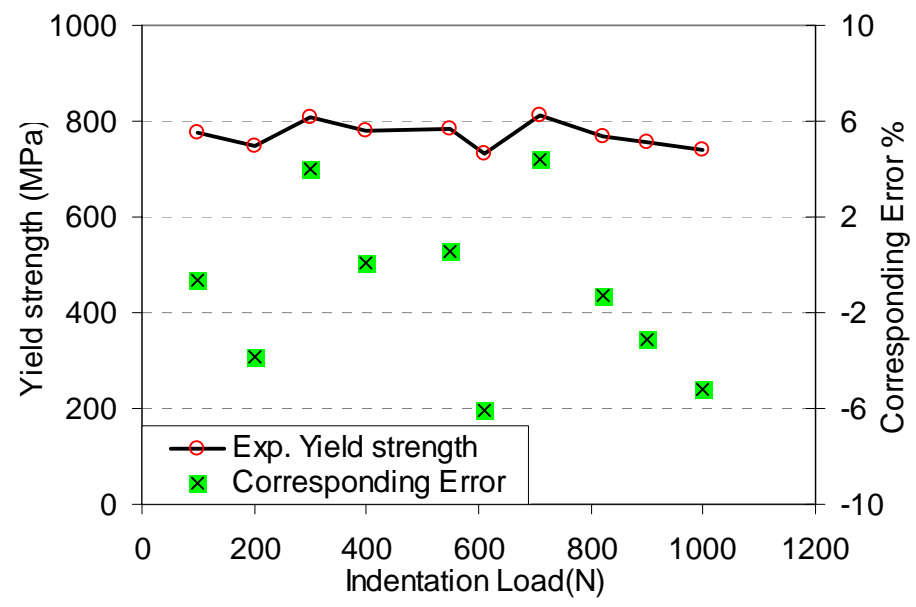

Figure 4.24 Results of yield strength (Average: $770 \mathrm{MPa}$ ) and corresponding error

\section{Indentation stress-strain vs. stress-strain curve}

Since contact radii from direct measurement of the residual indents are available, the post-yielding stress-strain data can also be quantified using Tabor's empirical relation. In this case, it is found a constrain factor 2.9 can make the better fit with the known stress-strain curve. As shown in Figure 4.25, the average yield strength (assuming Young's modulus is known) and indentation stress-strain data are plotted against true stress-strain curve.

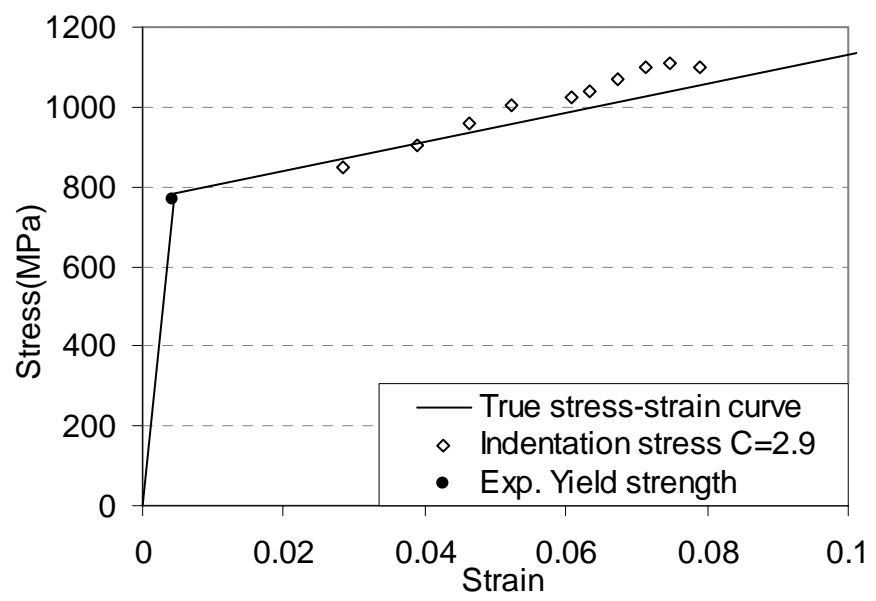

Figure 4.25 Indentation stress-strain vs. true stress-strain of IN783 superalloy 


\section{CHAPTER 5}

\section{TRANSPARENT INDENTER MEASUREMENT}

An in-situ Transparent Indenter Measurement (TIM) system is developed for material mechanical property evaluation based on spherical indentation. It is built through integrating a transparent indenter and a phase-shifting Twyman-Green interferometer into the indentation system. The TIM system directly measures the out-of-plane deformation and contact radius during indentation process. The derived elastic recovery is then used to calculate Young's modulus as described in \$3.4 of Chapter 3. The continuously measured contact radii are used to establish the material post-yielding true stress-strain relation using Tabor's empirical relation. Besides, the highly sensitive, in-situ and real-time measurement of the out-of-plane deformation may find new applications on the quality inspection of material surface conditions.

\subsection{Through Indenter Measurement (TIM) system}

\section{Optical schematic of integrated indentation system}

Figure 5.1 shows the optical principle of the TIM system of which a Twyman-Green ${ }^{[71]}$ interferometer and spherical indentation system are integrated together. A $2 \mathrm{~mm}$ diameter transparent indenter is attached to a non-polarizing cubic beam splitter. The two stacked cubic beam splitters are used to transfer load, incident light and viewing images during indentation process. The integrated Twyman-Green interferometer is used to directly measure the indentation-induced out-of-plane deformation. By introducing the phase shifting technique, the spatial resolution of measurements is further enhanced. It also improves the TIM system automation.

As shown in Figure 5.1, the polarization beam splitter (PBS) insures that the incident laser beam has linear polarization as required. The beam incident to the specimen has the polarization direction perpendicular to the paper and is reflected by the specimen back to the imaging system without changing its polarization direction. The reference beam passes through a quarter waveplate twice, thus its polarization direction is changed 90 degree. The polarization direction of these two beams is now mutually perpendicular with each other. The phase shifting is introduced as these two laser beams entering the Liquid Crystal Variable Retarder (LCVR) (Detailed information on how the LCVR is configured is described in Chapter 4). A polarizer is used to view the fringe pattern in the imaging system. 


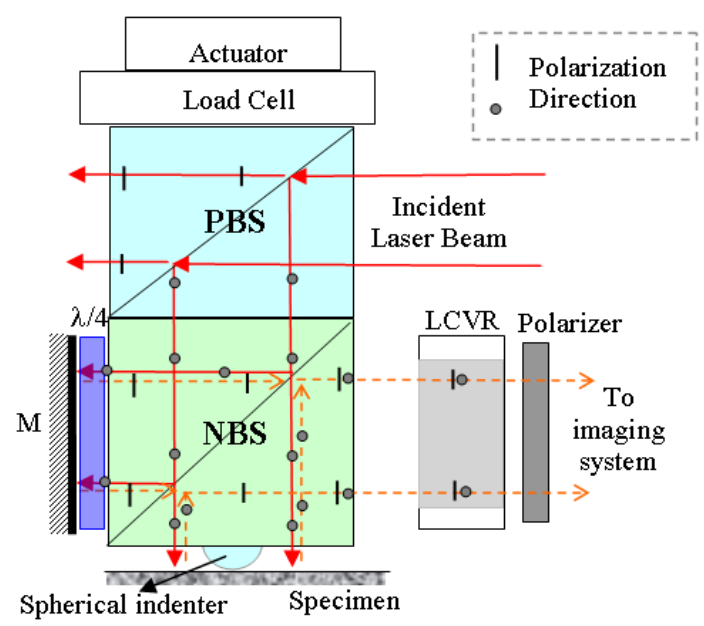

PBS: Polarization cubic beam-splitter, NBS: Non-polarization cubic beam-splitter,

LCVR: Liquid crystal variable retarder, M: Reference mirror, $\lambda / 4$ : quarter waveplate

Figure 5.1 Schematic optical principle of the TIM system

\section{Laboratory setup}

Figure 5.2 shows the TIM system laboratory setup. The TIM optical indenter is mounted on a heavy duty loading frame. The specimen with a high quality surface is placed on an adjustable heavy duty stage, which provides fine adjustment before indentation tests. Whenever load is applied, no stage adjustment should be performed. Instead, the reference mirror can be adjusted to perform the similar task. For the image acquisition and phase-shifting control, similar to residual deformation measurement, one Meadowlark Optics ${ }^{\mathrm{TM}}$ D2040 digital interface and National Instruments ${ }^{\mathrm{TM}}$ frame grabber (PCI-1408) are used with customized software.

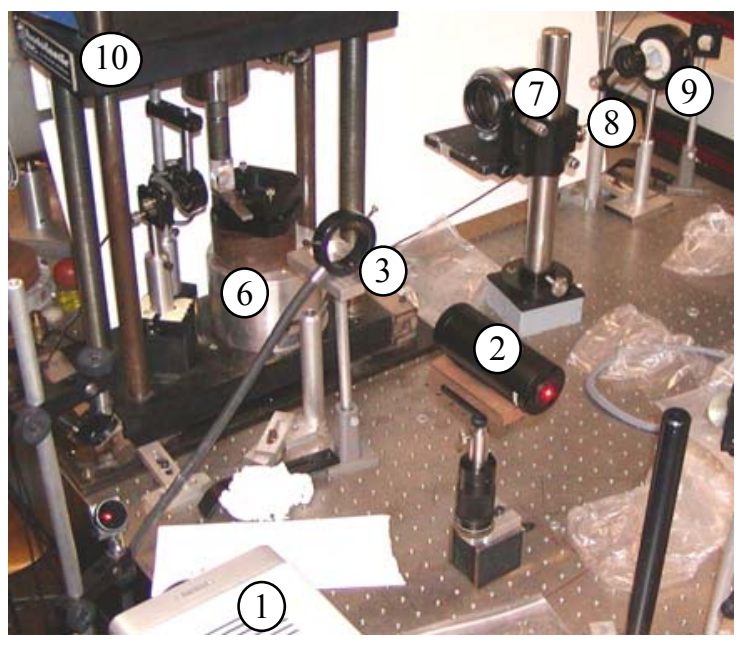

(a) Overview of the laboratory setup

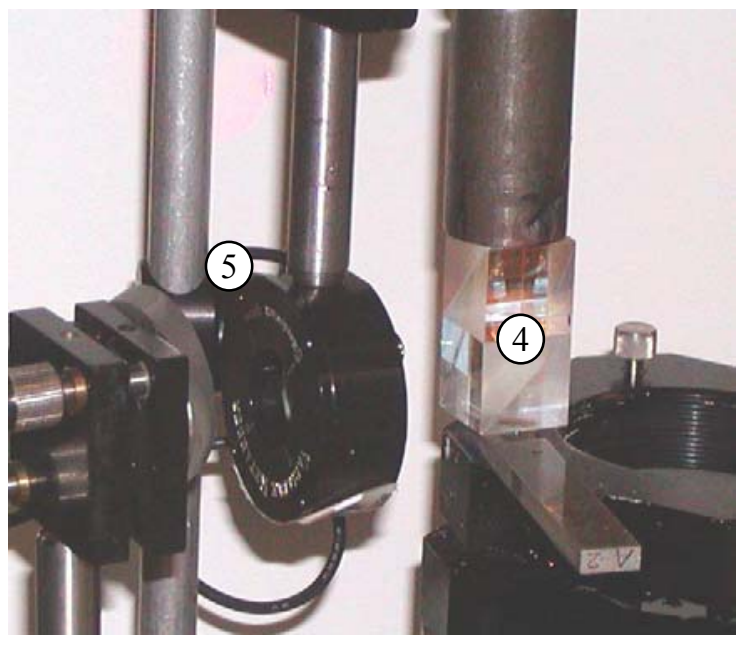

(b) TIM indenter head

1. HeNe Laser, 2. Beam expander, 3. Ring light source, 4. indenter head, 5. $\lambda / 4$ waveplate and reference mirror, 6. Specimen holder, 7. Imaging lens, 8. LCVR, 9. Polarizer, 10. Loading frame

Figure 5.2 TIM system laboratory setup 


\section{Testing procedures}

As shown in Figure 5.3, a typical TIM test involves two loading-unloading procedures at the same indentation spot. The first loading-unloading procedure is aimed at the measurement of Young's modulus, and the second is aimed at the determination of the material yield strength and post-yielding stress-strain data.

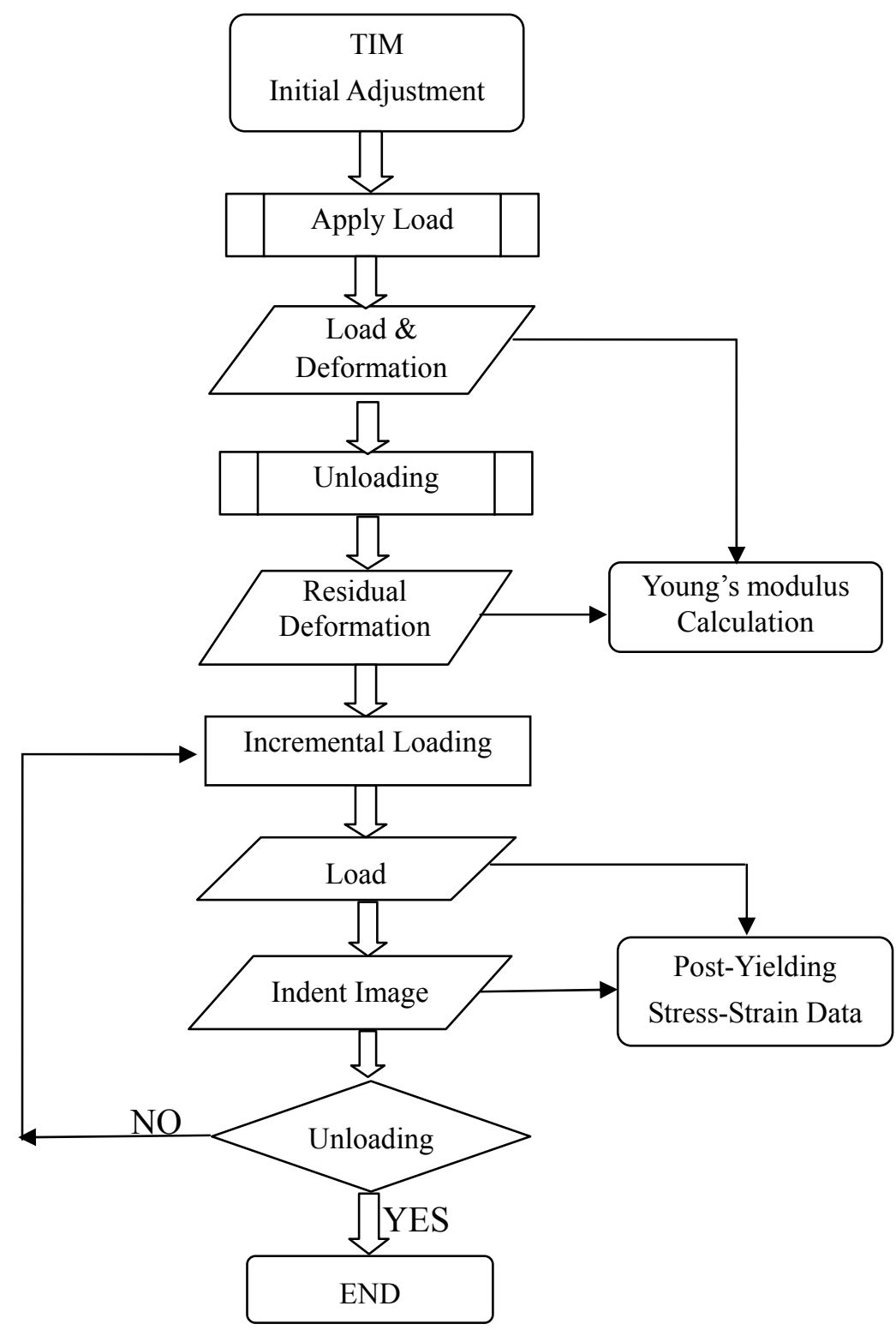

Figure 5.3 Testing procedures

At the first loading-unloading procedure, a small load is applied and the in-situ out-of-plane deformation is measured in real time. Then the load is released and the corresponding residual out-of-plane deformation is measured again. From the loading and residual out-of-plane displacements, Young's modulus is calculated following the procedure 
described in $\$ 3.4$ of Chapter 3.

After the first loading-unloading procedure, a sequence of monotonically increasing loads is applied at the same indented location. Correspondingly, using TIM system, the contact radii and applied loads are measured incrementally to obtain sufficient data, which are then used to establish the post-yielding stress-strain data using Tabor's empirical relation ${ }^{[1]}$ as well as determine the material yield strength.

\section{Testing materials}

The TIM tests are mainly focused on IN783 superalloy and A16061-T0 alloy samples. The initial residual stress is negligible in both of the materials. The IN783 superalloy samples are similar to those used on Chapter 4 , which had standard heat treatment $\left(1120^{\circ} \mathrm{C} / 1 \mathrm{hr} / \mathrm{AC}+\right.$ $845^{\circ} \mathrm{C} / 8 \mathrm{hrs} / \mathrm{AC}+720^{\circ} \mathrm{C} / 8 \mathrm{hrs} 50^{\circ} \mathrm{C} / \mathrm{hr} 620^{\circ} \mathrm{C} / 8 \mathrm{hrs} / \mathrm{AC}$ ) and a simplified bi-linear stress-strain curve. For standard A16061-T0, the stress-strain curve is $\sigma=205 \varepsilon^{0.2}$ (MPa) with Young's modulus of 69GPa and Poisson's ratio of 0.33 .

\subsection{Understanding the fringe pattern of TIM system}

Figure 5.4 shows a mounted $2 \mathrm{~mm}$ diameter transparent indenter attached to a cubic beam splitter. As shown in Figure 5.4(a), the corona (excessive glue around the indenter edge) will cause the formation of irregular fringes around the indenter. To conduct indentation test using the TIM method, the gap between the indenter and specimen surface is filled with index matching fluid to match the indenter material so that it behaves like a through window. Figure 5.4(b) shows the through indenter view using white light illumination.
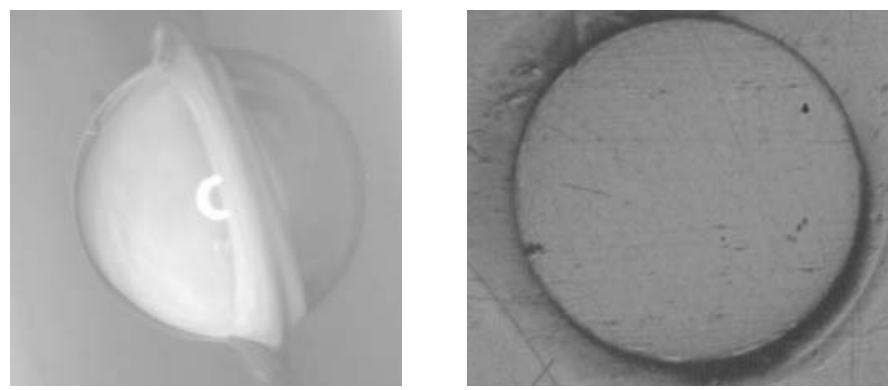

(a) 3D view of a mounted indenter (b) Through indenter view, white light illumination

Figure 5.4 View of a TIM indenter

The index changing rate of matching fluid is approximately $-0.0004 /{ }^{\circ} \mathrm{C}$. Without strict temperature control, initial fringe pattern is inevitable. However, using phase-shifting technique, this initial fringe pattern can be easily subtracted. The formation of the initial fringe patterns is discussed as followed.

As shown in Figure 5.5(a), $\mathrm{L}_{1}, \mathrm{~L}_{2}$ denoted the laser beam, and $0 \leq r \leq R=1 \mathrm{~mm}$. The relative optical path difference $(\mathrm{OPD})$ between $\mathrm{L}_{1}$ and $\mathrm{L}_{2}$ can be written as, 


$$
O P D_{\text {initial }}=2 \times\left(n_{\text {Indenter }} \sqrt{R^{2}-r^{2}}+n_{\text {index }_{-} \text {fluid }}\left(R-\sqrt{R^{2}-r^{2}}\right)\right)-2 \times n_{\text {Indenter }} R
$$

Figure 5.5(b) shows a computer generated fringe pattern using Equation (5-1) for a $2 \mathrm{~mm}$ diameter BK7 indenter with $n_{B K 7}=1.51509$ (at wavelength $632.8 \mathrm{~nm}$ ), and the index matching fluid, $n_{\text {index ffluid }}=1.5171$ (wavelength $\lambda=0.6328 \mu \mathrm{m}$ at $15^{\circ} \mathrm{C}$ ).
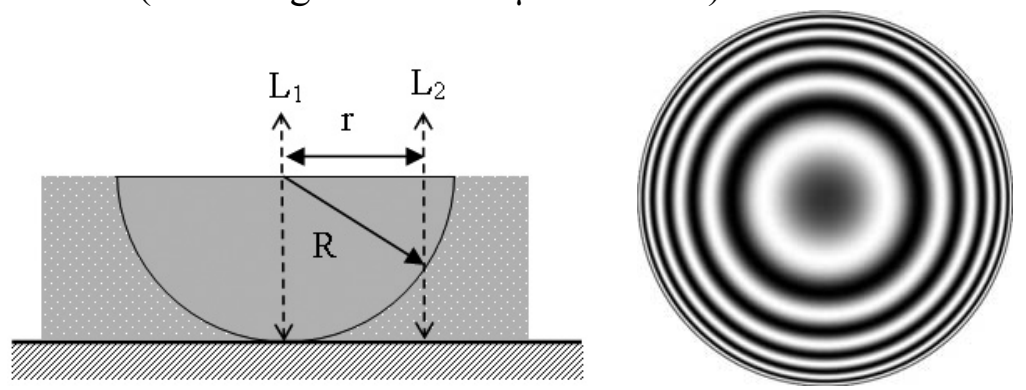

(a) Indenter in index matching fluid

(b) Theoretical initial fringe patterns

Figure 5.5 Initial fringe pattern in TIM system

Figure 5.6 shows the initial no load fringe patterns obtained by TIM system. Figure 5.6(a) to (d) show the four phase-shifted fringe patterns. Figure 5.6 (e) to (f) show the phase map and unwrapped phase map. The white circle indicates the $2 \mathrm{~mm}$ diameter indenter boundary. The outer denser irregular fringes are due to the excessive optical glue as showed in Figure 5.4(a).

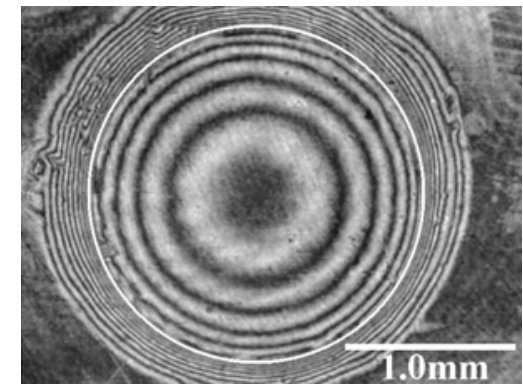

(a) $\phi=0$

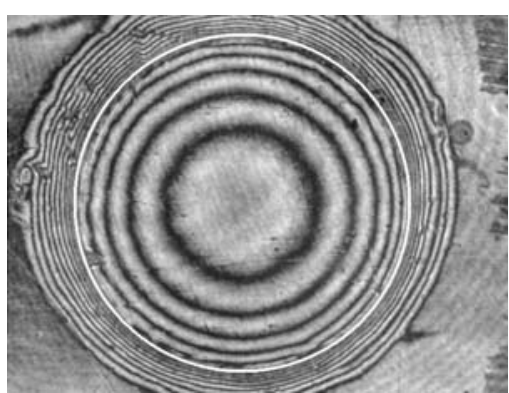

(d) $\phi=3 \pi / 2$

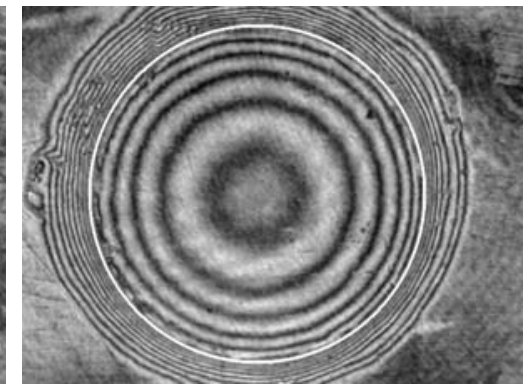

(b) $\phi=\pi / 2$

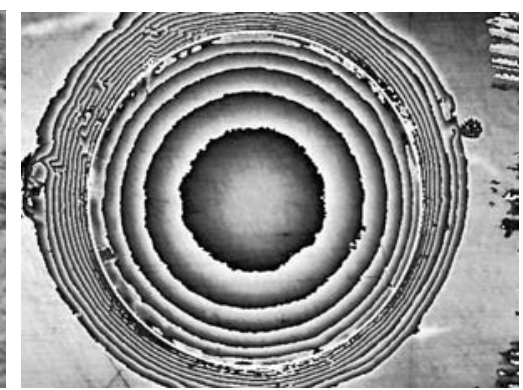

(e) Phase map

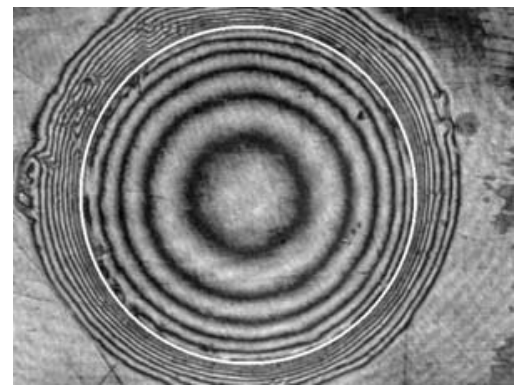

(c) $\phi=\pi$

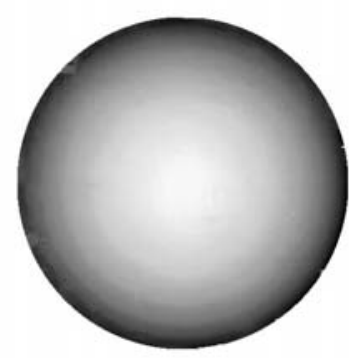

(f) Unwrapped phase

Figure 5.6 TIM system typical initial fringe patterns

Figure 5.7 shows the comparison between the experimental and theoretical initial fringe patterns. The numerically generated fringe patterns are overlaid on the top of the experimental 
fringe patterns. Except the boundary area, where the difference is expected due to the excessive glue, good agreement is achieved in the central region (about $80 \%$ of the indenter radius $R$ ).

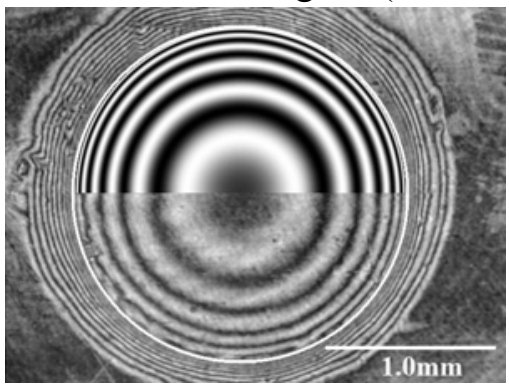

Figure 5.7 Comparison of experimental and theoretical initial fringe patterns

The initial fringe pattern affects the measurement of the out-of-plane displacement at each loading state. To obtain the real out-of-plane displacement, the initial fringe pattern must be subtracted from the measurement for each loading case. For Young's modulus measurement, since elastic recovery $\Delta w$ is use, the initial fringe pattern can be automatically canceled out.

Upon applying load, due to the out-of-plane displacement, the total OPD is written as,

$$
O P D_{\text {Loading }}=2 \times n_{\text {index_fluid }_{\text {flo }}} \times w_{\text {Loading }}+O P D_{\text {initial }}
$$

After unloading,

$$
O P D_{\text {UnLoading }}=2 \times n_{\text {index_fluid }_{\text {fli }}} \times w_{\text {unloading }}+O P D_{\text {initial }}
$$

Subtract Equation (5-2) from Equation (5-3), relative change of OPD is written as,

$$
\triangle O P D=2 \times n_{\text {index_fluid }_{\text {fl }}} \times\left(w_{\text {unloading }}-w_{\text {Loading }}\right)
$$

So the elastic recovery of out-of-plane deformation is,

$$
\Delta w=\frac{\Delta O P D}{2 \times n_{\text {index }_{-} \text {fluid }}}
$$

For a HeNe laser (632.8nm wavelength), each fringe pattern represents,

$$
\delta(\Delta w)=\frac{\lambda}{2 n_{\text {index }_{\text {fluid }}}}=\frac{632.8}{2 \times 1.5171} \mathrm{~nm}=208.6 \mathrm{~nm}
$$

It is obvious that the sensitivity has been increased due to the index matching fluid.

\subsection{Automatic cancellation of carrier fringes}

During loading, the specimen or indenter may be slightly inclined due to the loading frame rigidity and specimen mounting. Thus the fringes become no longer symmetric, as typically shown in Figure 5.8. There are two methods to solve this problem. One is to adjust the reference mirror to restore the symmetry. Another is to use the axisymmetric feature of the indenter to automatically cancel out the inclination-induced un-symmetric carrier fringes.

In a real indentation test, it is difficult to make the perfect alignment so that the fringe 
patterns are totally carrier-free. Usually, one or less carrier fringe may still present even after fine adjustment of the reference mirror. Thus, it is also necessary to find a solution to take care of this small carrier.

Under the coordinate system in Figure 5.8(a), the carrier-induced artificial displacement field can be described as,

$$
w=w_{\text {real }}+a_{L} x+b_{L} y+c_{L}
$$

Where $a, b$ and $c$ are constant coefficients and subscript $L$ denotes loading status.

Using the new coordinate systems as shown in Figure 5.8(b), corresponding displacement fields in quadrants 1 to 4 can be described as,

$$
\begin{aligned}
& w_{q 1_{-} L}=w_{L}+a_{L} x+b_{L} y+c_{L} \\
& w_{q 2_{-} L}=w_{L}-a_{L} x+b_{L} y+c_{L} \\
& w_{q 3_{-} L}=w_{L}-a_{L} x-b_{L} y+c_{L} \\
& w_{q 4_{-} L}=w_{L}+a_{L} x-b_{L} y+c_{L}
\end{aligned}
$$

At unloading state, the coefficient $a, b$ and $c$ of carrier fringes are different, i.e.

$$
\begin{aligned}
& w_{q 1_{-} u}=w_{u}+a_{u} x+b_{u} y+c_{u} \\
& w_{q 2_{-} u}=w_{u}-a_{u} x+b_{u} y+c_{u} \\
& w_{q 3_{\_} u}=w_{u}-a_{u} x-b_{u} y+c_{u} \\
& w_{q 4 \_u}=w_{u}+a_{u} x-b_{u} y+c_{u}
\end{aligned}
$$

Where $u$ denotes the unloading status.

Equations (5-8) to (5-11) are subtracted by Equations (5-12) to (5-15), and the results are added,

$$
\Delta w_{q 1}+\Delta w_{q 2}+\Delta w_{q 3}+\Delta w_{q 4}=4 \Delta w+4 \Delta c
$$

The elastic recovery can also be written as,

$$
\Delta w=\frac{1}{4}\left(\Delta w_{q 1}+\Delta w_{q 2}+\Delta w_{q 3}+\Delta w_{q 4}\right)-\Delta c
$$

Equation (5-17) shows that the carrier can be automatically cancelled out if the elastic recovery is averaged in four center-symmetric directions. 


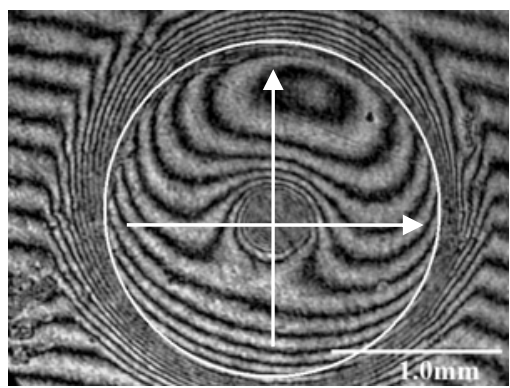

(a) Loading state with carrier

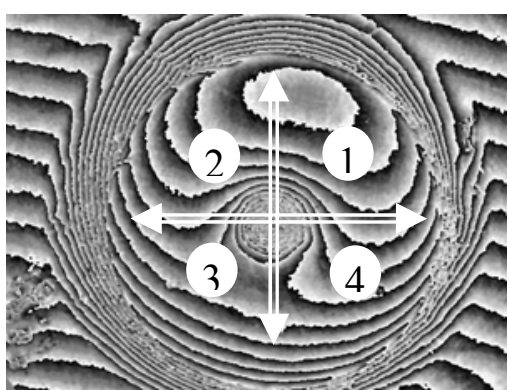

(b) Phase map at loading state

Figure 5.8 Carrier fringe patterns during loading-unloading

\subsection{TIM system testing results}

For a TIM test, the typical fringe patterns, phase map and corresponding white light image at loading state are shown in Figure 5.9. Under very small load, the profile inside the contact boundary is even measurable. From all the fringe patterns and phase maps, it clearly shows that on the interferometric images, the contact radius is difficult to measure, especially under small loading. So the white light or single laser beam (no reference beam presented) illumination is preferred when contact radius measurement is desired. In all the images, the white circles denote the indenter boundaries. In Figure 5.9(e), the black-dashed circle marks $80 \%$ of the indenter radius. As showed previously, for data inside this circle, the excessive glue effects can be considered negligible.

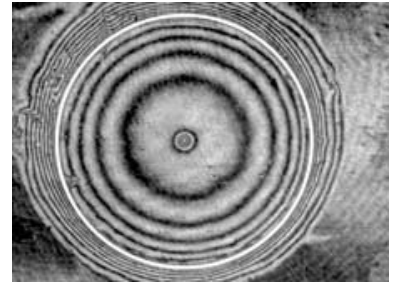

(a) step 1

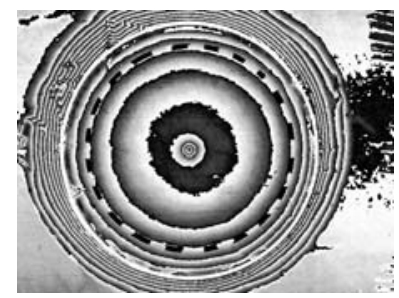

(e) phase map

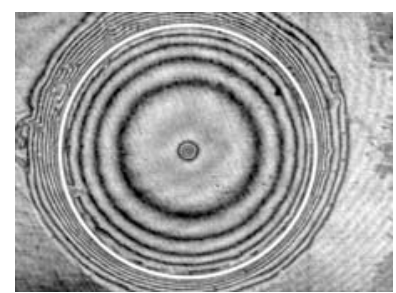

(b) step 2

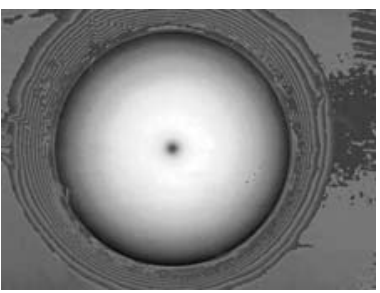

(f) unwrapped phase

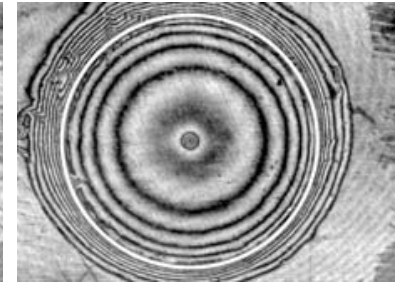

(c) step 3

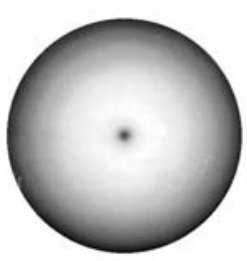

(g) Outside cleaned

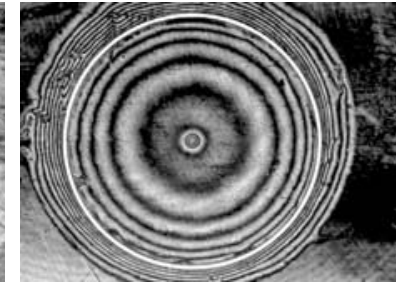

(d) step 4

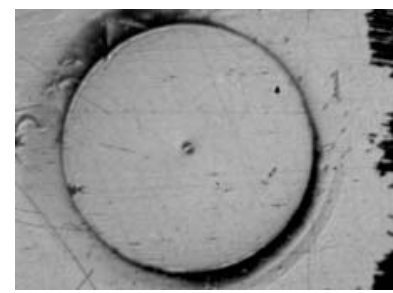

(h) white light image

Figure 5.9 TIM system typical fringe patterns, phase and image at loading

\section{Elastic recovery and Young's modulus}

Figure 5.10(a) shows the typical fringe patterns at loading state for a TIM test on an IN783 specimen, where outer white-lined circle denoted the indenter boundary. Figure 5.10(b) shows 
the corresponding phase map. Figure 5.10(c) shows the image for contact radius measurement. Figure 5.10(d) shows the typical fringe patterns at unloading state and Figure 5.10(e) shows the corresponding phase map. Figure 5.10(f) shows the image for contact radius measurement at unloading state. For current imaging system, no noticeable change in the contact radius measurement is found between loading and unloading state.

A phase map is first unwrapped to make it continuous. Then it is transformed to displacement regarding Equation (5-6). The elastic recovery was calculated by subtracting corresponding displacement fields. The results are shown in Figure 5.11(a). Using Equation (3-27), elastic recovery was changed to $\Delta w \sim t$ relation as shown in Figure 5.11(b) and (c) for the flat-end punch model and spherical indentation model, respectively. The slope $m$ of a chosen region is then calculated. The corresponding region is marked in Figure 5.11(a). Young's modulus is then calculated based on Equation (3-29). Table 5.1 showed the results of Young's modulus calculation.

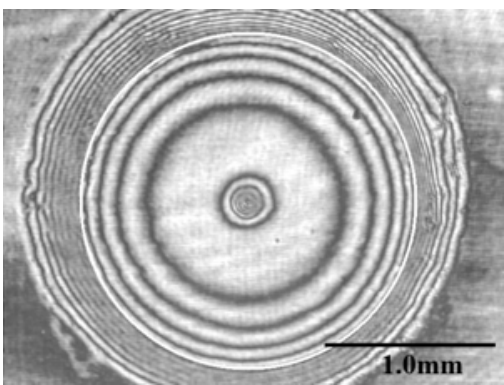

(a) Loading fringe pattern, $27.6 \mathrm{~N}$

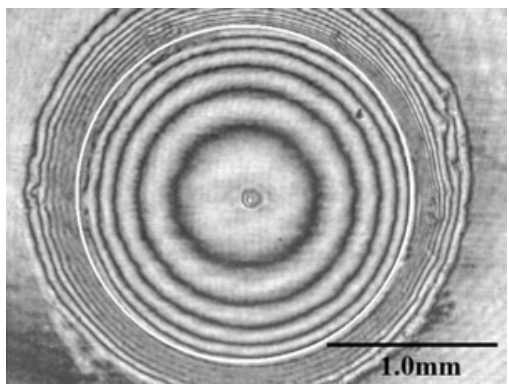

(d) Unloading fringe pattern

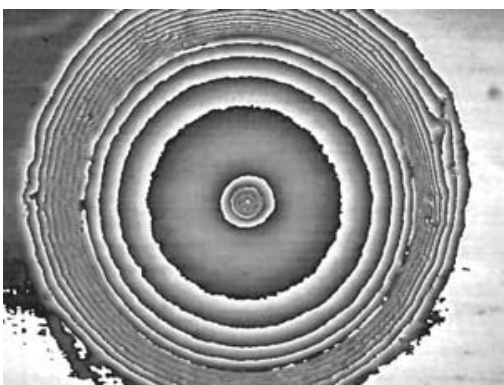

(b) Phase map

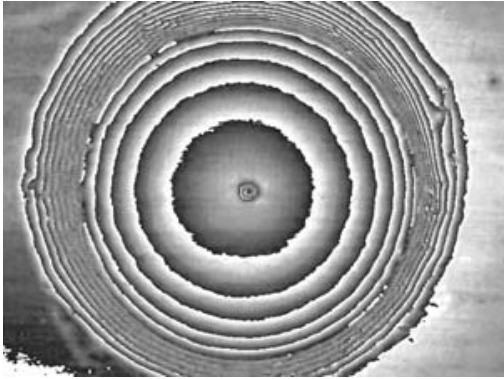

(e) Phase map

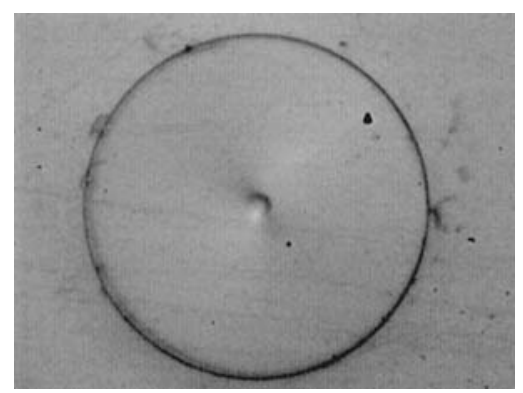

(c) Contact radius

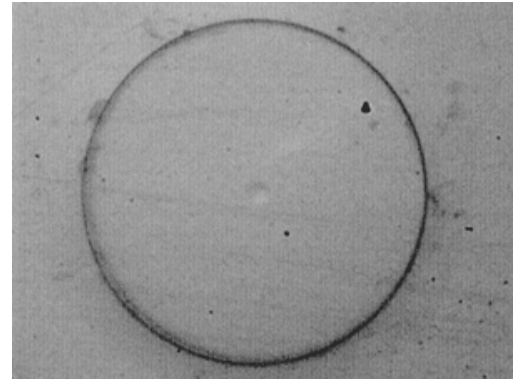

(f) Contact radius

Figure 5.10 Indentation of IN783 superalloy

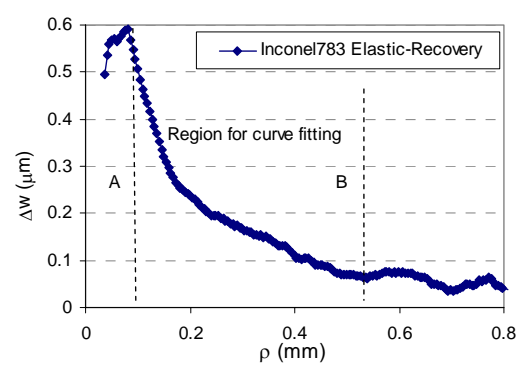

(a) Elastic recovery

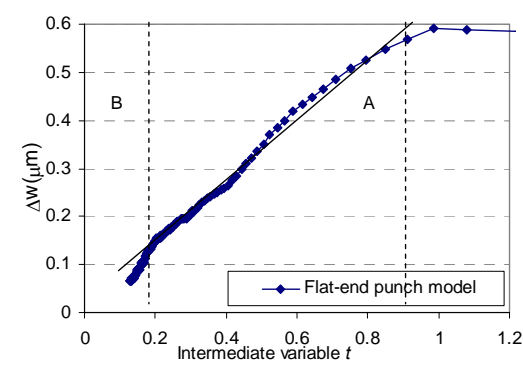

(b) $\Delta w \sim t$ relation in flat-end punch model

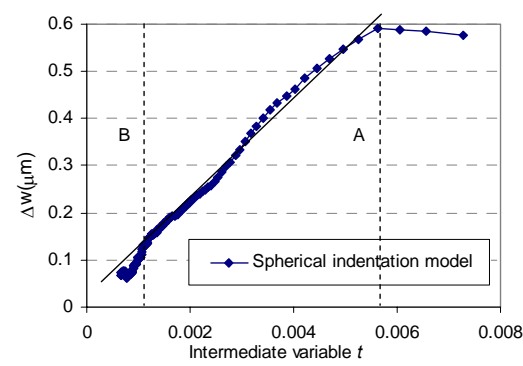

(c) $\Delta w \sim t$ relation in spherical model

Figure 5.11 Experimental elastic recovery and $\Delta w \sim t$ relation for IN783 
Figure 5.12 shows the loading and unloading fringe patterns, phase maps and contact radius images of a typical TIM test for A16061-T0. The carrier fringes are partially cancelled by adjustment of the reference mirror, but still, one or two carrier fringes is presented. By averaging the elastic recovery in four center symmetric directions, the carrier can be automatically canceled. The result is shown in the elastic recovery plot in Figure 5.13. For the $\Delta w \sim t$ plots shown in Figure 5.13(b) and (c), the slope $\mathrm{m}$ of a chosen region is also calculated as marked between A and B. Young's modulus is calculated and the results are shown in Table 5.1.

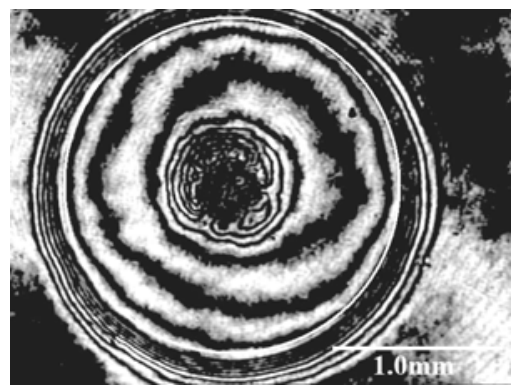

(a) Loading fringe pattern, $40 \mathrm{~N}$

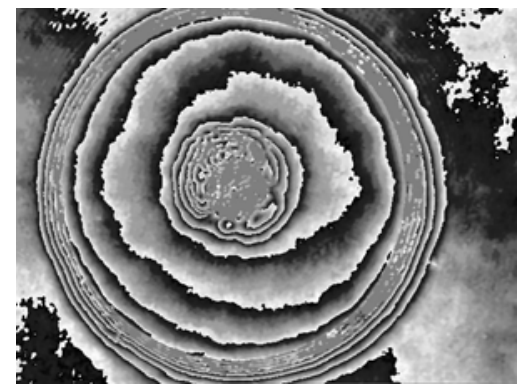

(b) Phase map

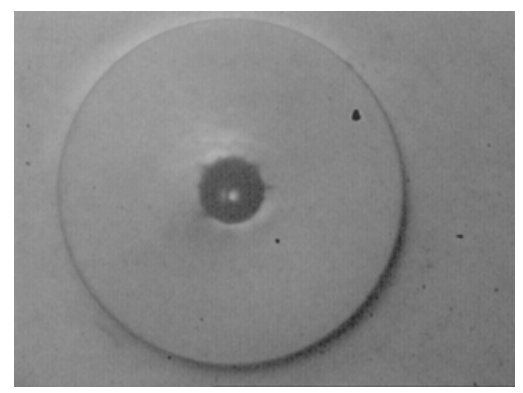

(c) Contact radius

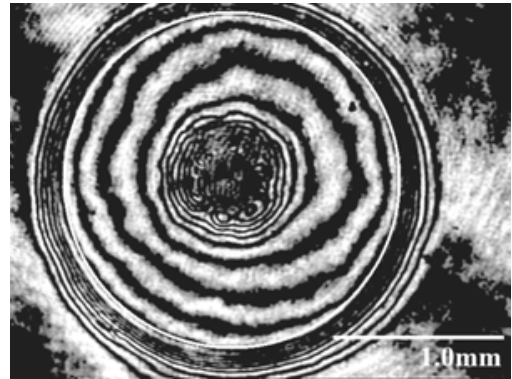

(d) Unloading fringe pattern

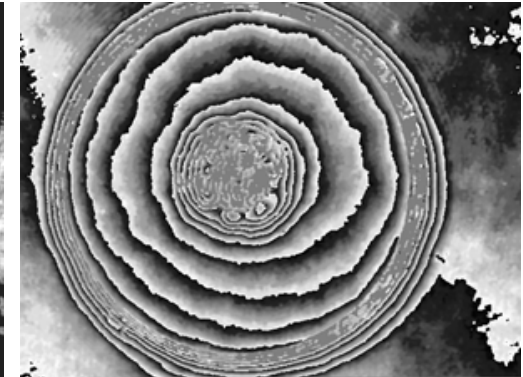

(e) Phase map

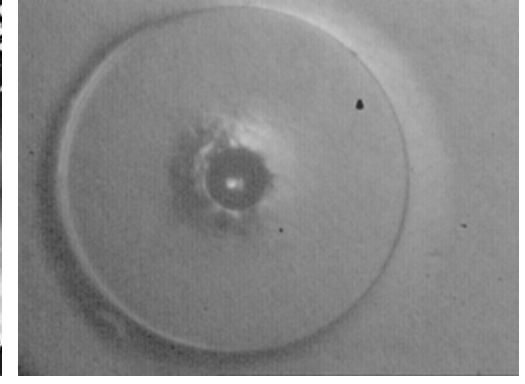

(f) Contact radius

Figure 5.12 Indentation of Al6061-T0
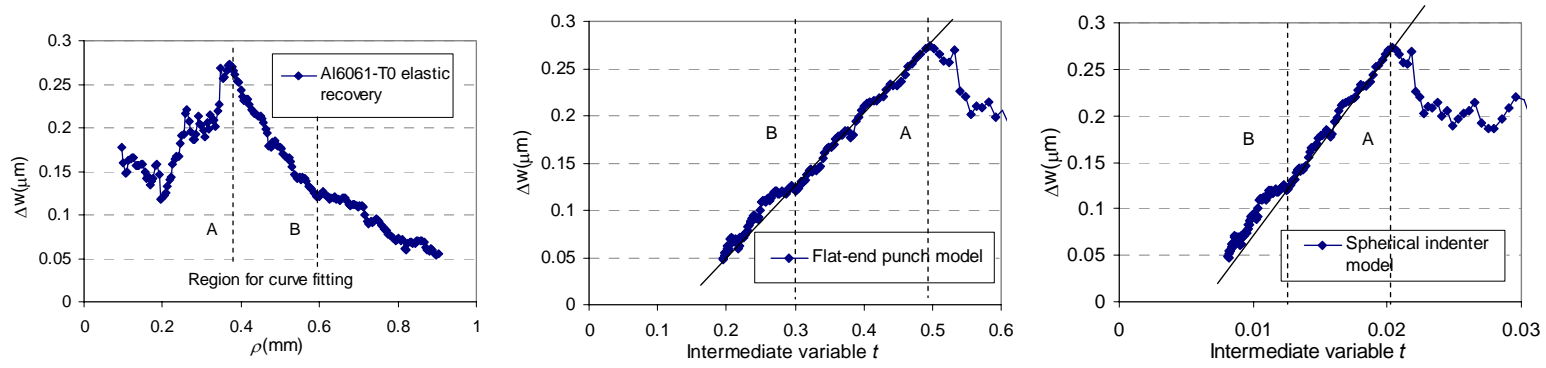

(a) Elastic recovery

(b) $\Delta w \sim t$ relation in flat-end punch model

(c) $\Delta w \sim t$ relation in spherical model

Figure 5.13 Experimental elastic recovery and $\Delta w \sim t$ relation for Al 6061-T0 
Table 5.1 Experimental error percentage of Young's modulus calculation

\begin{tabular}{|l|l|l|l|l|}
\hline & \multicolumn{2}{|l|}{ IN783 (177.3GPa) } & \multicolumn{2}{l|}{ Al 6061-T0 (69GPa) } \\
\hline & Average & Max. Error & Average & Max Error \\
\hline Flat-end model & $187.0 \mathrm{GPa}$ & $\leq 6 \%$ & $72.6 \mathrm{GPa}$ & $\leq 6 \%$ \\
\hline Spherical model & $174.5 \mathrm{GPa}$ & $\leq 5 \%$ & $70.3 \mathrm{GPa}$ & $\leq 4 \%$ \\
\hline
\end{tabular}

\section{Contact radius and post-yielding stress-strain}

The continuously measured contact radii are used to determine material post-yielding stress-strain relation based on Tabor's empirical relation.

$$
\varepsilon=0.2 \frac{d}{D} \quad \sigma=\frac{P_{m}}{C}
$$

Where $P_{m}=\frac{\text { Load }}{\pi a^{2}}$ is the mean pressure, $d$ is the contact diameter, a is the contact radius, $D$ is the indenter diameter and $\mathrm{C}$ is the constraint factor.

For steel and copper alloy, $\mathrm{C}=2.8$ was used by Tabor ${ }^{[1]}$ with excellent agreement. However, in general, it is found to be strain hardening dependent. Taljat et al ${ }^{[48]}$ suggested an analytical method to determine the constraint factor, and correlated it to the strain hardening coefficient, thus make the Tabor's empirical relation more general.

Figure 5.14 shows the typical in-situ measurement of contact radius for IN783 superalloy samples with increased loads. Due to its high strength, a $2 \mathrm{~mm}$ sapphire indenter is used to make larger load indentation. From the plot of post-yielding stress-strain relation as shown in Figure 5.15 , it is found that a constraint factor of 2.9 produces better results.

For A16061-T0, both the sapphire and regular BK7 indenter can be used. Figure 5.16 shows the typical in-situ measurement of contact radius with increased loads. For this material, a smaller constrain factor 2.8 is used to better match the post-yielding stress-strain curve.

Since all the images are acquired through the transparent indenter, so it is easy to check the indenter condition after each indentation test. From the residual interferential or white light illumination pictures, the damage can be instantly checked if there is. The indentation tests showed that there is no apparent damage for BK7 indenters for loading up to $300 \mathrm{~N}$ (Sometime it can even go up to $800 \mathrm{~N}$ ). For sapphire indenter, the load can be increased up to $1000 \mathrm{~N}$ without apparently damaging the indenter.
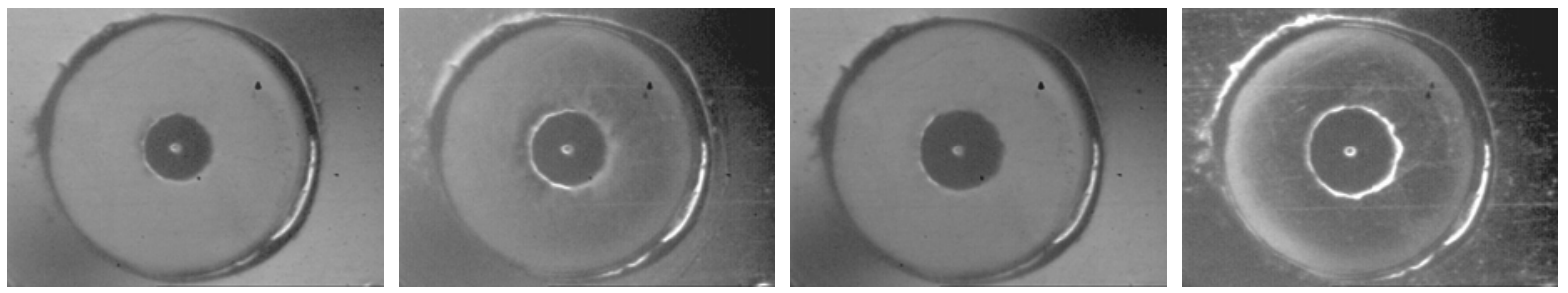
Figure 5.14 Typical in-situ contact radius measurement for IN783

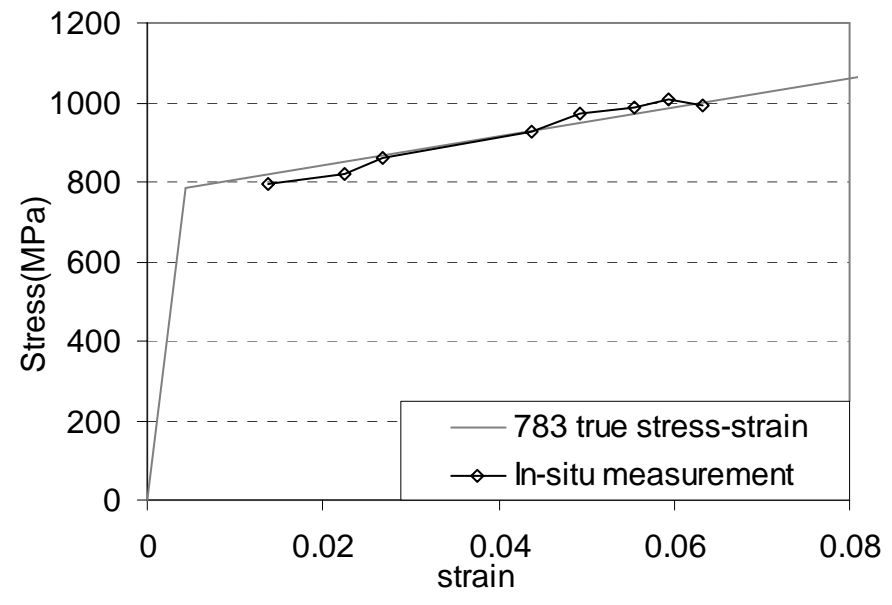

Figure 5.15 IN783 post-yielding stress-strain data

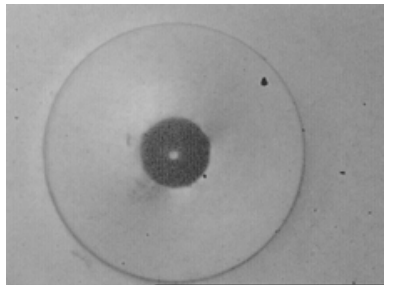

$69.1 \mathrm{~N}$

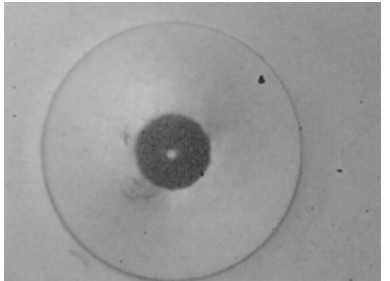

$84.0 \mathrm{~N}$

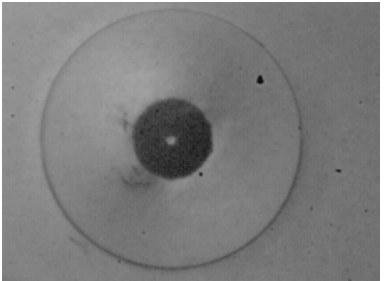

$95.1 \mathrm{~N}$

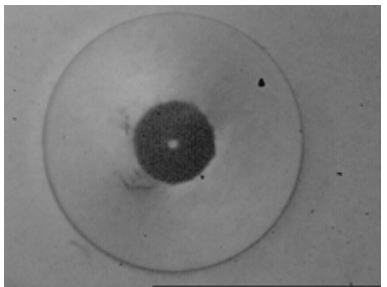

$99.6 \mathrm{~N}$

Figure 5.16 Typical in-situ contact radius measurement for Al6061-T0

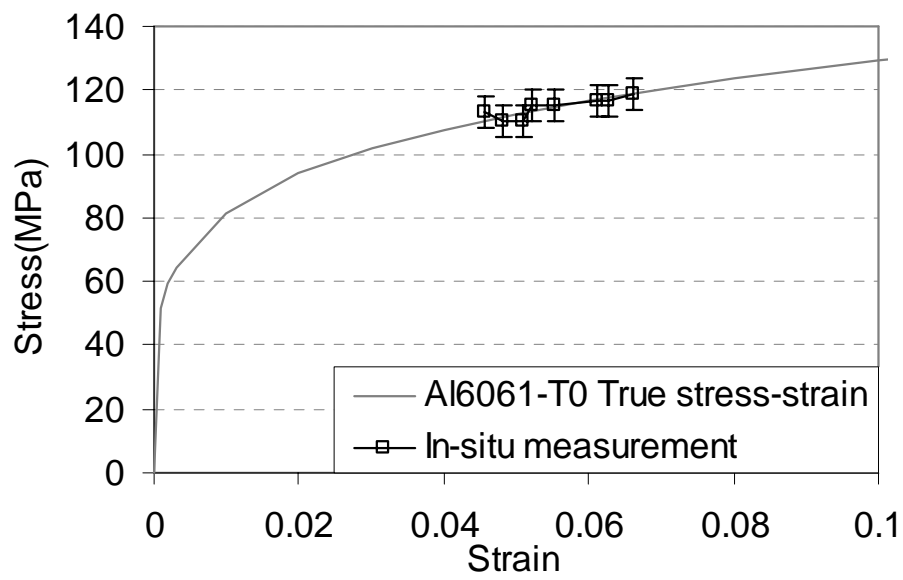

Figure 5.17 Al6061-T0 post-yielding stress-strain data 


\section{Strain hardening and yield strength}

If the material stress-strain relation follows the power law relation, using the TIM system, it is possible to determine the yield strength and strain hardening coefficient of the material from the continuous measured indentation loads and contact radii.

$\sigma=A \varepsilon^{n}$

By curve fitting Equation (5-19), parameter $A$ and $n$ can be determined from the post-yielding stress-strain data obtained in previous section.

Apply logarithm to both side of Equation (5-19),

$\ln \sigma=\ln A+n \ln \varepsilon$

Thus $n$ can be calculated from the slope of $\ln \sigma-\ln \varepsilon$ plot.

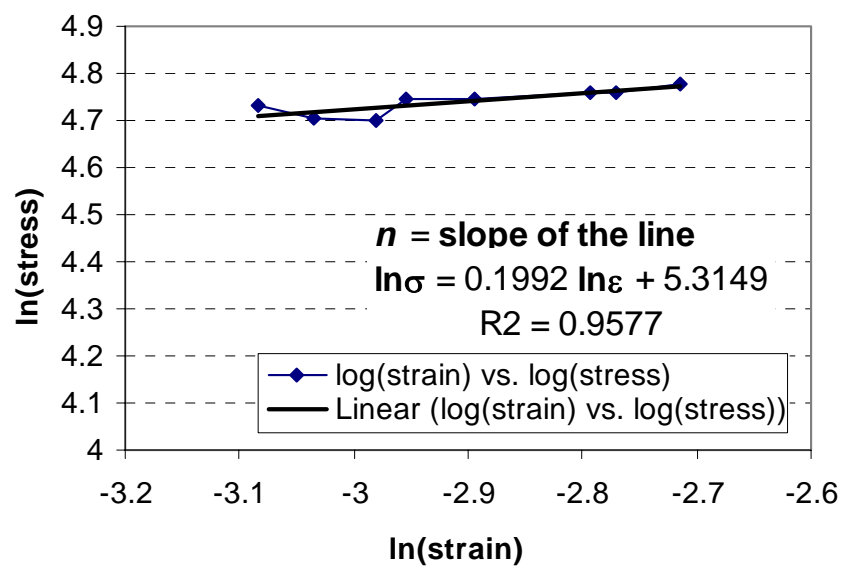

Figure 5.18 Calculation of strain hardening coefficient, Log-log plot

Then from the plot of $\sigma-\varepsilon^{n}$, the strength coefficient $\boldsymbol{A}$ can also obtained from the slope of a line through origin. The results show good agreement with known values.

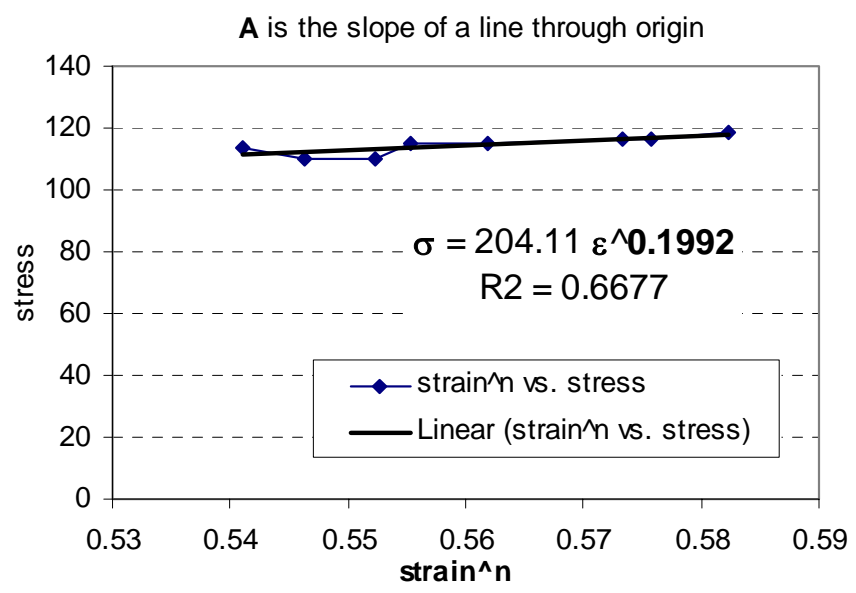

Figure 5.19 Calculation of strength coefficient A 
Since the general form of Tabor's relation can be derived from Meyer's law (See Equation (2-8)), the strain hardening coefficient $\boldsymbol{n}$ can also be derived from any form of Meyer's law. The following equations from Chapter 2 show several different forms of Meyer's law, see more details in the literature of Meyer hardness on Page 7.

$$
\begin{aligned}
& P=k d^{n^{\prime}} \approx k d^{n+2} \\
& P=D^{2} A\left(\frac{d}{D}\right)^{n^{\prime}}=D^{2} A\left(\frac{2 a}{D}\right)^{n+2}=2^{n+2} A \frac{a^{n+2}}{D^{n}}=k \frac{a^{n+2}}{D^{n}}
\end{aligned}
$$

If desired, the material yield strength can also be determined from the stress-strain curve of $\sigma=A \varepsilon^{n}$ based on the measured Young's modulus.

\section{Material surface condition inspection}

The high sensitivity out-of-plane deformation in-situ monitoring capability makes the TIM system an alternative tool for material surface quality inspection/assessment. This can be potentially useful for fracture/failure inspection of layered materials or materials with thin film coating.

A simple test on A12024-T3 with a thin layer (about $0.1 \mathrm{~mm}$ ) of PC-6C epoxy has been conducted to discover the potential. Figure 5.20 shows typical testing results. Figure 5.20 (a) to (c) shows the real-time fringe patterns with increased indentation loads. Figure 5.20(d) shows the fringe patterns after adjustment of the reference mirror and most of carrier fringes are removed. From the irregular fringe patterns showed in Figure 5.20(c), it clearly showed that debonding happened due to indentation load. Figure 5.20(e) and (f) are white light images at corresponding loads. It should be noted that from the white light pictures, it is almost impossible to check the debonding because out-of-plane deformation is too small to be noticed.

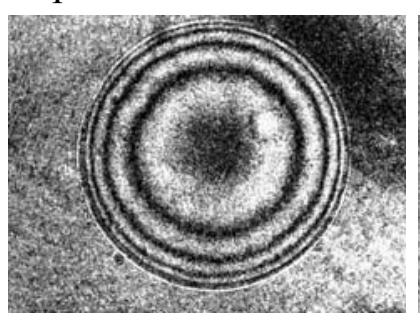

(a)

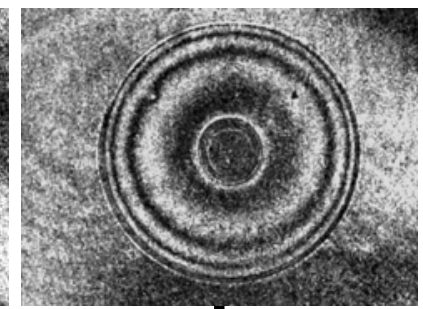

(b)

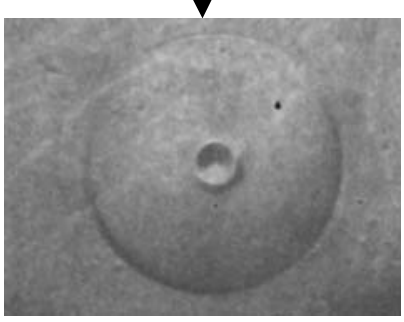

(e)

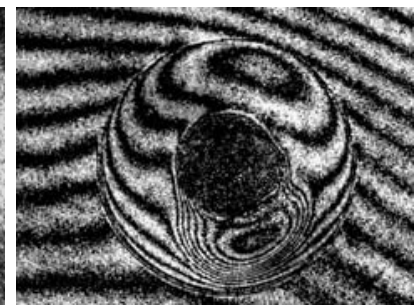

(c)

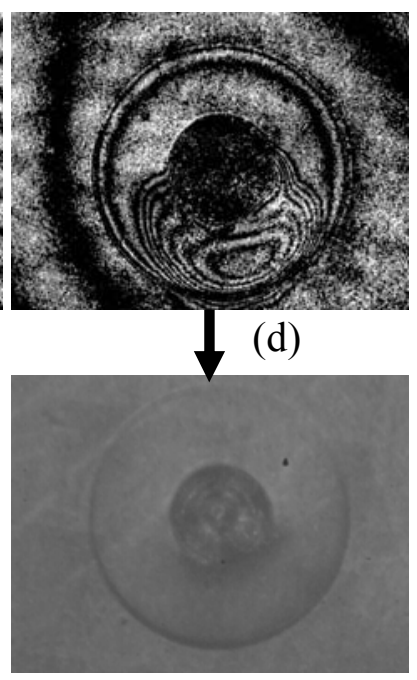

(f)

Figure 5.20 Real-time in-situ monitoring of Al2024-T3 with thin layer coating 


\subsection{Discussions on Portable TIM system design}

A fully automated and portable TIM system can be used for on-site material property evaluation and surface inspection. Some changes may benefit this unit for easy setup and testing. This part discusses some possible changes in order to build a portable TIM system.

\section{Index matching fluid holder}

For non-upright indentation tests, a suitable index matching fluid holder is necessary to hold the index matching fluid in position without leaking.

A schematic design of the index matching fluid holder is shown in Figure 5.21. The cylindrical thin-walled rubber or plastic is attached to the beam splitter. Since the gap between beam splitter and the specimen is only $1 \mathrm{~mm}$ or less, this soft holder is able to hold the index matching fluid even in the non-upright position. The cross section of this holder should be the shape shown in Figure 5.21 or similar, which may introduce minimum resistance to the indentation. A tiny hose may also be attached, or embedded to a slot grooved on the beam splitter. This hose can be connected to a syringe or similar equipment to control the injection or withdrawal of the index matching fluid.

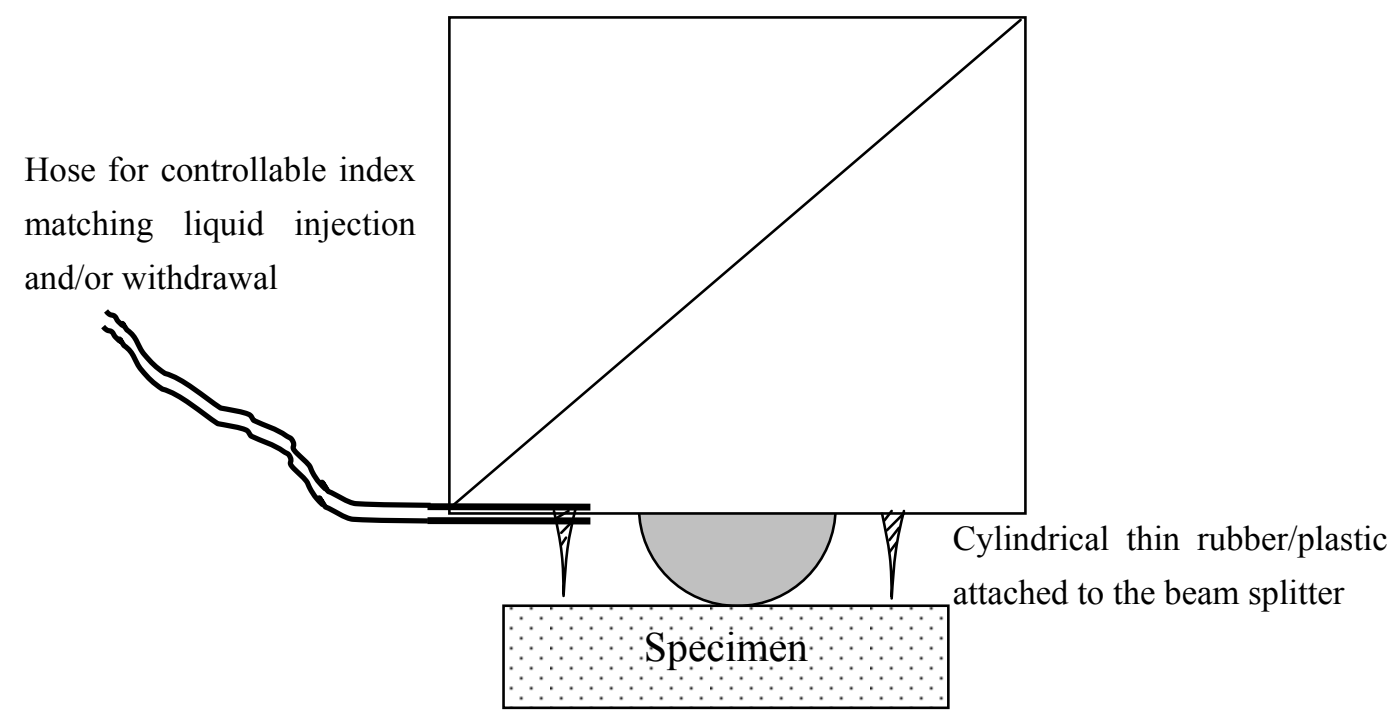

Figure 5.21 Index matching fluid holder

\section{Automatic reference beam blocking}

With the fringe pattern presented, it is difficult to measure the contact radius. It is desired to use a single laser beam illumination, but the reference beam must be blocked in this case. In order to control the reference beam, replace the quarter waveplate in Figure 5.1 by a Liquid Crystal Variable Retarder (LCVR-2). As shown in Figure 5.22, by changing the applied voltage 
to the LCVR-2, the polarization of the reference beam is highly controllable through the LCVR digital controller and computer. For the out-of-plane deformation measurements, the polarization direction is changed so that it is perpendicular to that of the specimen beam. If the reference beam needs to be blocked, change the voltage of LCVR-2, so the polarization direction of the reference beam is perpendicular to that of the polarizer. In the imaging system, the polarizer will block the reference beam, thus no fringe pattern is presented which makes the measurement of contact radius very convenient.

Moreover, this change can also speed up the image acquisition since all the image acquisition can be done in one step with automatic computer control. For each step, the imaging system will basically acquire 5 images. First four is related to the phase-shifting, and the last is used for contact radius measurement which is acquired through controlling the LCVR-2 for blocking reference beam. For each image acquisition cycle, the total time will be,

$$
\begin{aligned}
\mathrm{T}_{\text {one cycle }}=\mathrm{T}_{\mathrm{LCVR}-1}+\mathrm{T}_{\text {Fringe pattern 1 }}+\mathrm{T}_{\mathrm{LCVR}-1}+\mathrm{T}_{\text {Fringe pattern 2 }}+ \\
\\
\mathrm{T}_{\mathrm{LCVR}-1}+\mathrm{T}_{\text {Fringe pattern 3 }}+\mathrm{T}_{\mathrm{LCVR}-1}+\mathrm{T}_{\text {Fringe pattern 4 }}+\mathrm{T}_{\mathrm{LCVR}-2}+\mathrm{T}_{\text {Picture }}
\end{aligned}
$$

The response time for LCVR is very short (in the order of milliseconds), so the time for one step data acquisition is basically the time for acquiring 5 images.

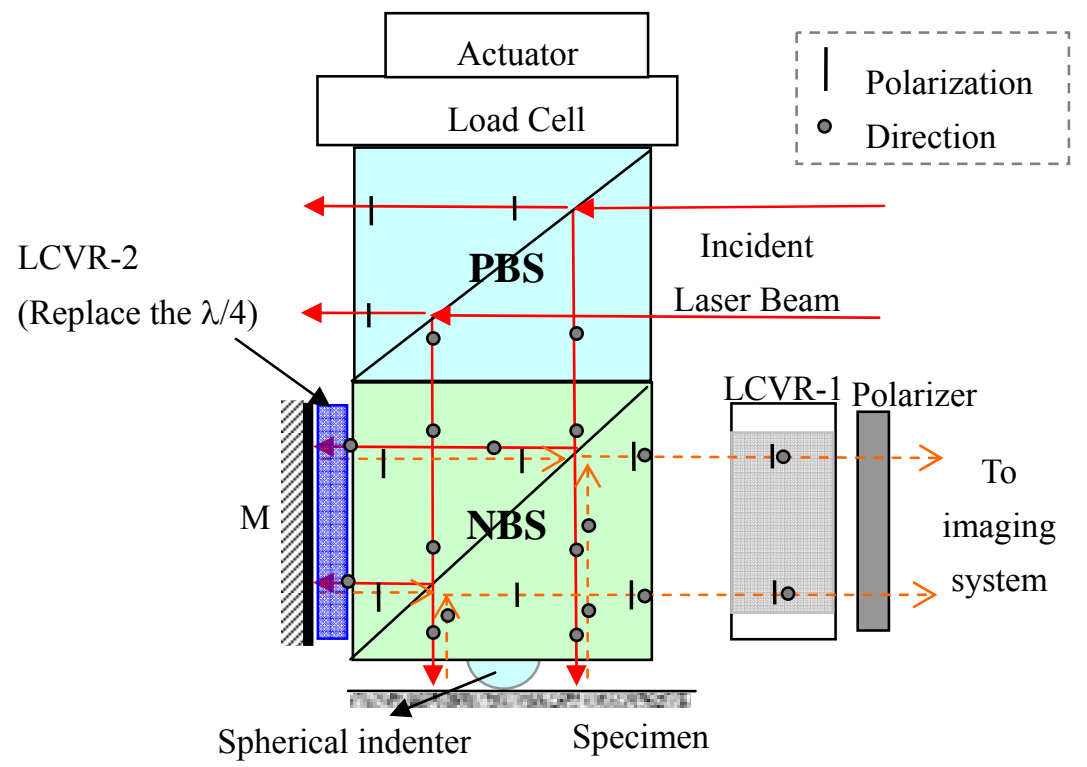

Figure 5.22 Modified TIM system

Another solution is to use white light source instead of blocking the reference beam. In that case, an in-line design of white light source (Use either same as incident laser beam or embedded in the imaging system) is necessary. Also, there must be a mechanism to control the presence of the laser beam.

\section{Variation of TIM system}

In a portable TIM system, the polarization direction of the incident laser beam can be 
carefully aligned. So it is not necessary to use a polarization beam splitter (PBS). A non-polarization beam splitter work as better as the PBS. Using a non-polarization beam splitter, the imaging system can also be modified using vertical design. As shown in Figure 5.23, imaging system is put on top of the beam splitter instead of using the reflection of the first NBS. The LCVR-1 and imaging system is put inside the supporting of the indenter. This system can be made very compact. The drawback of this design is the light usage is only half of the original one since the reflect light passes one more beam splitter.

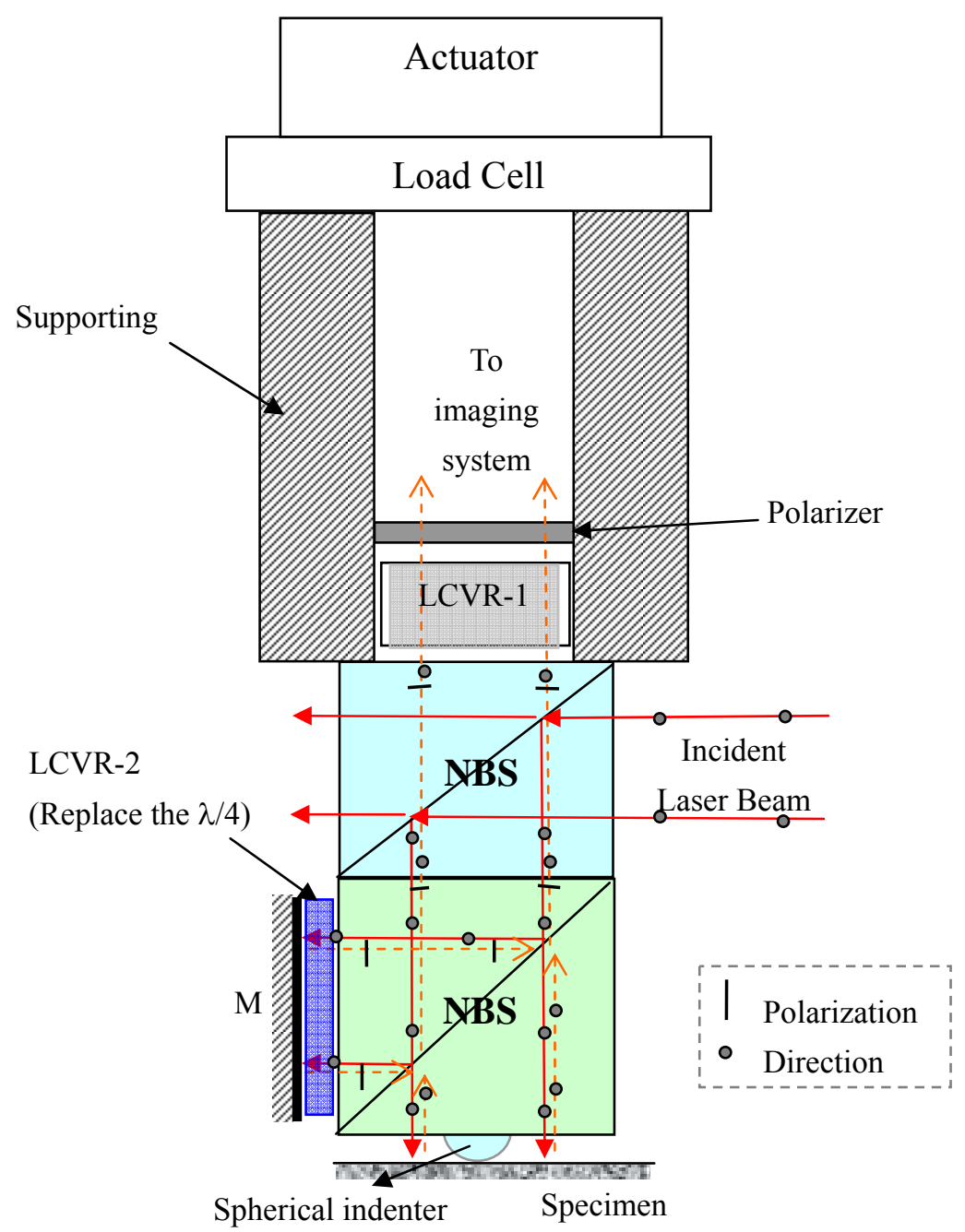

Figure 5.23 Compact design of TIM system 


\section{CHAPTER 6}

\section{SUMMARY, CONCLUSIONS AND FUTURE PERSPECTIVES}

\subsection{Summary}

The needs for techniques to study the mechanical properties of thin-film coating and small volume of material have rekindled the interest in the indentation technique. In the literature, most of the material properties, such as Young's modulus, yield strength, strain hardening coefficient, fracture toughness, ultimate strength, residual stress, indentation creep, coating failure/debonding, strain rate effects etc, have been reported to correlated to indentation hardness parameters. However, the indentation hardness testing is a complex mechanical process, which makes the general indentation problem difficult to solve analytically. Most of the understanding of this process is based on experiments and finite element simulations. Thus experimentally accurate measurement of indentation parameters is critical in the determination of the surface mechanical properties using the indentation technique. The contact area (or contact radius for spherical indentation) is an important parameter. As of now, the direct measurement is still not available during indentation process. Further more, for the indentation-induced surface deformation, the discussions are mostly limited to the residual profile/out-of-plane deformation, while in-plane deformation carries more surface stress, strain information. Thus it is necessary to bridge the deformation fields and mechanical properties.

This dissertation explored the spherical indentation-based material mechanical property evaluation. A TIM system is proposed and developed to accurate measure the indentation parameters, including the contact radius and out-of-plane deformation. How to use the surface deformation is also discussed and procedures to obtain yield strength and Young's modulus are developed. During the development of the TIM system and corresponding algorithms, a series of finite element analyses were conducted. The surface deformation analyses are performed to correlate surface deformation to various material properties.

Experimentally, the residual deformation fields are first extensively studied on samples of IN783 superalloy. Both in-plane and out-of-plane residual deformation are obtained using a combined phase-shifting moiré and Twyman-Green interferometer. The qualitative analyses of the residual surface stress distribution are conducted and the relation between the load and contact radius, load and elastic-plastic boundary are explored.

The featured indentation system using transparent indenter and integrated optical interferometry is developed to conduct in-situ real-time measurement of the indentation-related parameters, including out-of-plane deformation and direct contact radius measurement. Based on the finite element analyses, a procedure is developed to ascertain Young's modulus directly 
from the elastic recovery measurements. The continuous measurement of the contact radius makes it suitable to apply Tabor's empirical relation in one indentation test. How to extend the load, contact radius measurement to more mechanical properties, such as yield strength and strain hardening parameter is further discussed. Finally, the potential of this new indentation design in thin-film coating or layered material is discussed.

\subsection{Conclusions}

The following concluding remarks and comments are drawn from the work in this dissertation:

(1) A Transparent Indenter Measurement (TIM) system is successfully developed. It features the continuous, real-time measurement of the contact radius and out-of-plane deformation. The procedure of a fully automated TIM testing is proposed. The tests using a laboratory prototype verified the feasibility of the TIM system.

(i) An optical indenter is developed by integrating a phase-shifting Twyman-Green interferometer using Liquid Crystal Variable Retarder. (ii) Successfully explained the formation of the initial fringe pattern using Optical Path Difference analysis. (iii) A procedure of automatically cancellation of the inclination-induced carrier fringes is proposed. This method can only be used for homogenous materials since it averages the displacements in four center-symmetric directions. (iv) The potential applications for the new TIM system are discussed in the layered material and thin-film coating materials. (v) Further improvement of the TIM system is proposed for a portable type TIM system.

(2) The indentation-induced surface residual deformation on samples of IN783 superalloy is successfully measured and analyzed. It confirmed the yield strength can be accurately calculated from the surface residual in-plane deformation. A combined phase-shifting moiré and Twyman-Green interferometer is developed. It puts the two interferometers together, so they share the same imaging system with fully phase-shifting capability.

(3) The 2D finite element analyses are successfully conducted. Based a series of finite element simulations, the procedures for the measurement of the yield strength and Young's modulus are proposed. The results are comparable with the experimental results.

(i) The effects of the friction coefficient are studied. It is found with increased friction both contact radius and elastic-plastic boundary become smaller, but the change of contact radius is negligible. (ii) The relation between the loads and elastic-plastic boundaries are comparable with the relation between the loads and contact radii. (iii) 
It is confirmed that both the flat-end punch model and spherical indenter model can be used to calculate the Young's modulus. Based on the elastic theoretical solutions of indentation process, a procedure is proposed to solve the over-determination elastic recovery data. This procedure helps to choose a proper data region and thus can reduce the noise and improve the results furthest. (iv) The results from different indenter show that the indenter rigidity has little influence on the calculation of Young's modulus using the proposed method.

\subsection{Recommendations and future perspectives}

Future studies may be directed to further refine the TIM system and implement a fully automated and portable TIM system, which may be very helpful to the on-site material property evaluation and surface condition inspection.

(1) Implementation of a fully automated TIM system. This should include an integrated imaging system, automated indentation system following the procedure outline at Chapter 5. Also, a better index matching solution holder will make the testing process very smooth and neat. For applications where only contact radius measurements are interested, how to simplify the TIM system so that it can dramatically reduce the complexity of a TIM system may be very useful.

(2) Software-wise, a fully automated TIM system should be equipped with fully functional control software. Development of such software is critical since the control and data process needs to follow the procedure described in Chapter 5, and it should also have a flexible control with configurable parameters for easily extended functions in the future.

(3) Based on the accurate measured contract radius, how to improve the literature-presented correlations should also be studied. How to determine the constraint factor $\mathrm{C}$ in a general case should also be studied.

(4) For the thin-film and layered material, study the correlation between indentation load and fracture toughness when debonding happens.

(5) With the real-time out-of-plane deformation, is that possible to evaluate the brittleness and ductility of a given material?

(6) In case the in-plane deformation is desired and zero-thickness grating is difficult to apply, how can we use transferred grating? How to estimate the error introduced by transferred gratings? This may be very useful for large structures such as bridge and aircraft. 


\section{REFERENCES}

[1]. D. Tabor, The Hardness of Metals, Clarendon Press, Oxford, 1951

[2]. George E. Dieter, Mechanical metallurgy, McGraw-Hill book company, 1988

[3]. Website: http://www.gordonengland.co.uk/hardness/

[4]. Website: http://www.hardnesstesters.com, Newage Testing Instruments, Inc

[5]. Stefan Frank, Transpyramidal Indentation Viewing - New possibilities for mobile hardness testing, 15th World Conference on Non-Destructive Testing, October 2000 in Rome, http://www.krautkramer.com, and http://www.geinspectiontechnologies.com/products/HardnessTesters/index.html, product literature

[6]. Alcala, J.; Barone, A.C.; Anglada, M. Source; Influence of plastic hardening on surface deformation modes around Vickers and spherical indents, Acta Materialia, v 48, n 13, Aug, 2000, p 3451-3464

[7]. ASTM Standards

[8]. J. H. Westbrook, Am. Soc. Test. Mater. Proc., vol 57, pp873-897, 1957, ASTM Bull. 246, pp 53-58, 1960

[9]. M. C. Shaw and G. J. DeSalo, Trans. ASME, Ser. B; J. Eng. Ind., vol. 92, pp469-479, 1970; M. C. Shaw and G. J. DeSalvo, Met. Eng. Q., vol. 12, pp.1-7, May 1972

[10]. J. B. Cahoon, W. H. Broughton, and A. R. Kutzak, Metall. Trans., vol 2, pp. 1979-1983, 1971

[11]. Huang, Y; Xue, Z; Gao, H; Nix, WD; Xia, ZC; A study of microindentation hardness tests by mechanism-based strain gradient plasticity, JOURNAL OF MATERIALS RESEARCH, 15 (8): 1786-1796 AUG 2000

[12]. A. Nadai, Plasticity: a mechanics of the plastic state of matter, McGraw-Hill book company, Inc, 1931

[13]. W. F. Chen; D. J. Han; Plasticity for Structural Engineers, 1995, Gau Lih Book Co., Ltd. ISBN: 957-584-321-5

[14]. Abaqus Documentation, HKS, Inc.; http://www.abaqus.com

[15]. W. C. Oliver; G. M. Pharr, An improved technique for determining hardness and elastic modulus using load and displacement sensing indentation experiments, JOURNAL OF MATERIALS RESEARCH, 7 (6):1564-1583 June 1992

[16]. Ian N. Sneddon, 'The Relation between Load and Penetration in The Axisymmetric Boussinesq Problem For A Punch Of Arbitrary Profile', Int. J. Engng Sci, Vol. 3, pp. 47-57, 1965

[17]. J. L. Loubet, J. M. Georges, J. M. Marchesini, and G. Meille, 'Vicker's Indentation Curves of Magnesium Oxide (MgO)', J. Tribology, 106, 43, 1984

[18]. R. B. King, 'Elastic Analysis of Some Punch Problems for a Layered Medium', Int. J. Solids Structures, Vol. 23, No. 12, pp. 1657-1664, 1987

[19]. K. L. Johnson, 'The Correlation of Indentation Experiments', J. Mech. Phys. Solids, Vol. 18, pp. 115-126, 1970

[20]. A. K. Bhattacharya, W. D. Nix, Finite element simulation of indentation experiments, Int. J. Solids Structure, Vol 24, No. 9, pp. 881-891, 1988

[21]. A. K. Bhattacharya, W. D. Nix, Analysis of elastic and plastic deformation associated with indentation testing of thin films on substrates, Vol 24, No. 12, pp. 1287-1298, 1988

[22]. John H. Underwood, Residual-stress measurement using surface displacements around an indentation, Experimental Mechanics, Vol. 13 No.9 pp 373-380, 1973

[23]. Lin Lihua, Chen Ligong, Gu Mingyuan, FEM analysis of simplified model for residual stress measuring 
process with static indentation, Journal of Shanghai Jiaotong University, Vol. 32, No. 2, Feb 1998

[24]. Chen Huaining, Lin Quanhong, Chen Jing, Yu Zhfu, Zhao Yinghua, On plastic zone around an indentation during stress determination by impact indentation method, Transactions of the China welding institution, Vol.22, No.5, October 2001

[25]. Yu Zhefu, Zhao Yinghua, Chen Huaining, Impacted indentation method of measuring residue stress, Journal of Shenyang Arch. And Civ. Eng. Univ., Vol.17, No.3, Jul. 2001

[26]. A. K. Bhattacharya, W. D. Nix, Finite element simulation of indentation experiments, Int. J. Solids Structure, Vol.24, No.9, pp881-891, 1988

[27]. A. K. Bhattacharya, W. D. Nix, Analysis of elastic and plastic deformation associated with indentation testing of thin films on substrates, Int. J. Solids Structure, Vol.24, No.12, pp1287-1298, 1988

[28]. S.I. Bulychev, V. P. Alekhin, M. Kh. Shorshorov, A. P. Ternovskii and G. D. Shnyrev, Determining Young's modulus from the indentor penetration diagram, Industrial laboratory, Vol.41, No.9, pp 1409-1412, Sep 1975(Translated from Zavodskaya Laboratoriya, Vol. 41, No.9, pp1137-1140, Sep 1975, Original article submitted Aug 1974)

[29]. J. B. Cahoon, W. H. Broughton, and A. R. Kutzak, Metall. Trans., Vol. 2, pp1979-1983, 1971

[30]. A. I. Lure, Three-dimensional problems in the theory of elasticity[in Russian], Gostekhizdat, Moscow(1955)

[31]. G. M. Pharr, W. C. Oliver, and F. R. Brotzen, J. Mater. Res. Vol.7, 613, 1992

[32]. K. L. Johnson, Contact Mechanics, Cambridge Univeristy Press, 1985

[33]. S. S. Chiang, D. B. Marshall and A. G. Evans, The response of solids to elastic/plastic indentation:I. Stresses and residual stress, J. Appl. Phys., Vol.53, No.9,pp.299-311, 1982

[34]. Bishop, R. F., Hill, R., and Mott, N. F., The theory of indentation and hardness tests, Proc. Phys. Soc., vol 57 , 1945, pp. 147-159

[35]. Hill, R., The mathematical theory of plasticity, Clarendon Press, Oxford, 1967, pp. 97-106

[36]. K. Komvopoulos, Finite element analysis of a layered elastic solid in normal contact with a rigid surface, Journal of Tribology, Vol. 110, p477-485, July 1988

[37]. K. Komvopoulos, Elastic-plastic finite element analysis of indented layered media, Journal of tribology, Vol.111, p430-439, July 1989

[38]. E. R. Kral, K. Komvopoulos, D. B. Bogy, Elastic-plastic finite element analysis of repeated indentation of a half-space by a rigid sphere, Journal of applied mechanics, Vol. 60, p829-841, Dec 1993

[39]. E. R. Kral, K. Komvopoulos, D. B. Bogy, Finite element analysis of repeated indentation of an elastic-plastic layered medium by a rigid sphere, part I: surface results, Journal of applied mechanics, Vol.62, p20-28, March 1995

[40]. E. R. Kral, K. Komvopoulos, D. B. Bogy, Finite element analysis of repeated indentation of an elastic-plastic layered medium by a rigid sphere, part II: subsurface results, Journal of applied mechanics, Vol.62, p29-42, March 1995

[41]. E. R. Kral, K. Komvopoulos, Three-dimensional finite element analysis of surface deformation and stresses in an elastic-plastic layered medium subjected to indentation and sliding contact loading, Journal of applied mechanics, Vol.63, p365-375, June 1996

[42]. Lubos Prchlik, Jan Pisacka, Sanjay Sampath, Deformation and strain distribution in plasma sprayed nickel-aluminum coating loaded by a spherical indenter, Material science and engineering A360, p264 274, 2003 
[43]. F. M. Haggag, Field Indentation Microprobe for structural integraity evaluation, U.S. Patent No. 4,852,397, August 1, 1989

Haggag, F. M., Nanstad, R. K., Hutton, J. T., Thomas, D. L., and Swain, R. L., "Use of Automated Ball Indentation to Measure Flow Properties and Estimate Fracture Toughness in Metallic Materials," Applications of Automation Technology to Fatigue and Fracture Testing, ASTM 1092, A. A. Braun, N. E. Ashbaugh, and F. M. Smith, Eds., American Society for Testing and Materials, Philadelphia, 1990, pp. 188-208

[44]. Website and technical literature library, http://www.atc-ssm.com/

Haggag, F. M. and Lucas, G. E., "Determination of Lüders Strains and Flow Properties in Steels from Hardness/Microhardness Tests," Metallurgical Transactions A, 14A, (1983), pp.1607-1613

[45]. M. Szutkowska, Fracture toughness measurement of WC-Co hardmetals by indentation method, Journal of advanced materials, Vol.31, No.3, July 1999

[46]. M. F. Doerner, W. D. Nix, A method for interpreting the data from depth-sensing indentation instruments, J. Mater. Res. 1(4), p601-609, Jul/Aug 1986

[47]. Yang-Tse Cheng, Che-Min Cheng, Can stress-strain relationships be obtained from indentation curves using conical and pyramidal indenters? J. Mater. Res., Vol.14, No.9, p3493-3496, Sep 1999

[48]. B. Taljat, T. Zacharia, New analytical procedure to determine stress-strain curve from spherical indentation data, Int. J. Solids structures, Vol. 35, No. 33, pp. 4411-4426, 1998

[49]. Hyung Yil Lee, Ball indenter utilizing FEA solutions for property evaluation, U.S. Patent No. 6,778,916, Aug. 17, 2004

[50]. H. A. Francis, Phenomenological Analysis of Plastic Spherical Indentation, Trans. ASME, 1976, P. 272

[51]. P. Au, G.E. Lucas, J.W. Sheckherd, and G.R. Odette, Flow property measurements from instrumented hardness tests, Proceedings of the third international conference, 11-13 February 1980, Salt Lake City, Utah

[52]. Rashid Kamel Abu Al-Rub, Material Length Scales in Gradient-Dependent Plasticity/Damage and Size Effects: Theory and Computation, Disseration, Louisiana State University, May 2004

[53]. James W. Dally, William F. Riley, Experimental Stress Analysis, $3^{\text {rd }}$ edition, McGraw-Hill, 1991

[54]. Daniel Post, Bongtae Han, Peter Ifju, High sensitivity moiré: experimental analysis for mechanics and materials, Springer, 1994

[55]. Handbook on experimental mechanics, edited by Albert S. Kobayashi, $2^{\text {nd }}$ edition, 1993

[56]. Colin A. Walker, A historical review of moiré interferometry, Experimental mechanics, 281-299, Dec. 1994

[57]. Zygo website, www.zygo.com, literature library

[58]. E Guan, Chuanyu Feng, Hong Miao, and Xiaoping Wu, An Assessment of Digital Speckle Correlation Method, Proceedings of the SEM Annual Conference on Theoretical, Experimental and Computational Mechanics, June 1999

[59]. Gary Cloud, Optical methods in Engineering,

[60]. J. W. Goodman, Introduction to Fourier Optics, McGraw-Hill, 1968

[61]. FENG Chuanyu, JIANG Zhen-yu, MIAO Hong, HE Shiping, A Correlative Region Algorithm for Phase Unwrapping, Journal of Experimental Mechanics(in Chinese), Vol.15, No.2, 2000

[62]. D.C.Ghiglia, G.A.Mastin \& L.A.Romero, Cellular-automata method for phase unwrapping, J.Opt.Soc.Am.1987, A4:267-280

[63]. J. M. Huntley, Noise-immune phase unwrapping algorithm, Appl. Opt., 1989, 28:3268 -3270

[64]. R.Crusack, J. M.Huntley \& H.T.Goldrein, Improved noise-immune phase-unwrapping algorithm, 
Appl.Opt., 1995, 34:781-789

[65]. Su, X., Xue, L., Phase unwrapping algorithm based on fringe frequency analysis in Fourier-transform profilometry, Optical Engineering, 2001, 40(4):637-643

[66]. Judge, T.R., Bryanston-Cross, P.J, Review of phase unwrapping techniques in fringe analysis, Optics and Lasers in Engineering, 1994, 21(4):199-239

[67]. Su, X., Chen, W., Fourier transform profilometry: A review, Optics and Lasers in Engineering, 2001, 35(5):263-284

[68]. http://www.eiaj.ch/v2/recherche prestation/publications/publications/, Lucien Falco, Automated Twyman-Green interferometer for micro-optical component characterization, 2004

[69]. http://www.sciences.univ-nantes.fr/physique/enseignement/english/michp.html, Michelson interferometer

[70]. B V Dorr'ýo, D Cernadas, C Trillo, A F Doval, C L'opez, MP'erez-Amor and J L Fern andez, A multiplicative analogical moiré phase-shifting Twyman-Green technique for Nd:YAG rod wavefront distortion measurement, Meas. Sci. Technol. 12 (2001) 103-110

[71]. Gary Cloud, Optical methods in Experimental Mechanics, part 8: Michelson Interferometry, Experimental Techniques, Vol.25, No.5, Sep/Oct 2003

[72]. J. F. Cardenas-Garcia, J. L.F. Freire, and W. -C. Wang, Status of Experimental Mechanics series part 3: Optical Methods, Experimental Techniques, Vol.25, No.5, Sep/Oct 2003

[73]. J. Guild, The interference systems of crossed diffraction gratings: theory of moiré fringes, Oxford at the Clarendon press, 1956

[74]. Christopher Palmer, Diffraction grating handbook, fifth edition, Thermo RGL, 2002

[75]. http://www.spectrogon.com

[76]. Kenneth R. Castleman, Digital image processing, Prentice Hall, Inc, 1998

[77]. http://www.ettemeyer.com/, ESPI and other products

[78]. http://www.photomechanics.com, PEMI products

[79]. Michael L. Basehore and Daniel Post, Displacement fields(u, w) obtained simultaneously by moiré interferometry, Applied optics, Vol.21, No.14/15, July 1982

[80]. F. A. La Porta, J. M. Huntley, T. E. Chung and R. G. Faulkner, High-magnification moiré interferometer for crack tip analysis of steels, Experimental mechanics, Vol.40, No.1, p90-95, March 2000

[81]. S. T. Lin, A new moiré interferometry for measuring in-plane displacement, Experimental mechanics, Vol. 41, No.2, p140-143, June 2001

[82]. B. Kang, X. Liu et al, High Temperature Moiré Interferometry Investigation of Creep Crack Growth of Inconel 783 - Environment and $\beta$-phase Effects, Materials Science \& Engineering A, p 205-213, A347 (2003)

[83]. B.S. -J, Kang, J. Skeen, K. Perry and Y.Y. Hung, Crack tip displacement measurements using automated phase shift moiré interferometry, 1997 SEM spring conference on experimental mechanics, pp311-312, Bellevue, WA, 1997

[84]. Ridha Kamil, Processing improvement of moiré interferometry for accurate crack-tip deformation measurement, master thesis, West Virginia University, 2001

[85]. NTPort SDK, http://www.zealsoftstudio.com

[86]. Anthony C. Fischer-Cripps, Introduction to contact mechanics, Springer, 2000

[87]. IN783 superalloys, http://www.specialmetals.com 


\section{VITA \\ Chuanyu Feng \\ chuanyufeng@yahoo.com chuanyufeng@gmail.com}

\section{EDUCATION}

$\mathrm{PhD}$ in Mechanical Engineering

West Virginia University

Morgantown, WV, 26506, U.S.A.

http://www.wvu.edu

M.E. in Solid Mechanics

University of Science and Technology of China

Hefei, Anhui, 230026, P.R. China

http://www.ustc.edu

B.S. in Theoretical and Applied Mechanics

1997

University of Science and Technology of China

Hefei, Anhui, 230026, P. R. China

http://www.ustc.edu

\section{EXPERIENCE}

Research Assistant, West Virginia University, 2000 2005

Engineer, HighTech Division, TongLian Group, Shenyang, China, 2000

Research Assistant, University of Science and Technology of China, 1997 2000

\section{ACADEMIC BACKGROUND}

Experimental stress analysis and Photomechanics (interferometry, moiré, ESPI, DIC / DISC, Particle Image Velocimetry (PIV) etc); Mechanical metallurgy;

Solid mechanics, theoretical and applied mechanics; Fracture mechanics;

Mechanical design, solid modeling and finite element analysis;

Software development, data / image acquisition, processing and analysis;

\section{SELECTED PUBLICATIONS}

\section{Conferences:}

Chuanyu Feng, Bruce S. Kang, In-situ Indentation TIM System and Material Mechanical Property Evaluation, Proceedings of the 2005 SEM Annual Conference and Exposition on Experimental and Applied Mechanics, Portland, 
Oregon, June 7-9, 2005.

Chuanyu Feng, Bruce S. Kang, Residual Surface Deformation Analysis in IN783 after Spherical Indentations, Proceedings of the 2005 SEM Annual Conference and Exposition on Experimental and Applied Mechanics, Portland, Oregon, June 7-9, 2005.

Bruce S. Kang, Chuanyu Feng, Kian Huat Tan, Long-time Wearing Performance of Ceramic Inserts in Continuous Galvanizing Line, Proceedings of the 2005 SEM Annual Conference and Exposition on Experimental and Applied Mechanics, Portland, Oregon, June 7-9, 2005.

B.S. Kang, C. Feng, J. H. Schneibel, Thermomechanical properties of molybdenum alloys with spinel particles, Proceeding of Twentieth Annual International Pittsburgh Coal Conference. Sep 15-19, 2003

He Shiping; Feng Chuanyu; Han Yun; Ma Li, An improved automated grid method, Proceedings of SPIE - The International Society for Optical Engineering, $3^{\text {rd }}$ International Conference on Experimental Mechanics,, V4537, 2001, p 305-308

E Guan, Chuanyu Feng, Hong Miao, and Xiaoping Wu, An Assessment of Digital Speckle Correlation Method, Proceedings of the SEM Annual Conference on Theoretical, Experimental and Computational Mechanics, p19-22, Cincinnati, Ohio, June7-9, 1999

\section{Journals:}

Bai, Yiru; Bai, Shiwei; Feng Chuanyu; Study and application of speckle correlation measurement method for displacement fields of model, Rock and Soil Mechanics (Chinese), p995-998, Vol.25, No.6, 2004

Feng Chuanyu, Wang Xiaowen, Han Yun, He Shiping, Deformation Measurement of Large Specimen With Hole Using Automated Grid Methods, Journal of Experimental Mechanics(Chinese), p116-122, Vol.01, No.1, 2002.

Feng Chuanyu, Han Yun, Ma Li, He Shiping, Grating Fourier Analysis and Computer Moiré Technique, Journal of China University of Science and Technology(Chinese), p179-183, Vol.31, No.2, 2001

FENG Chuanyu, JIANG Zhenyu, MIAO Hong, HE Shiping, A Correlative Region Algorithm for Phase Unwrapping, Journal of Experimental Mechanics(Chinese), p197-205, Vol.15, No.2, 2000 
Feng Chuanyu; He, Shiping; Wu, Xiaoping; A Study on the Measurement of Thermocapillary Flow Velocity Field, Journal of Experimental Mechanics(Chinese), p429-433, Vol.13, No.4, 1998

\section{COMPUTER SKILLS}
ABAQUS, Ansys; Pro/E, Solidworks;
$\mathrm{C} / \mathrm{C}++$ /Borland $\mathrm{C}++$ Builder, VC, MatLAB, LabVIEW, LabWindows/CVI;
MIL-Lite, IMAQ with numerous experiences on data, image acquisition

\section{INDEPENDENTLY DEVELOPED SOFTWARE TOOLS}

DISC Tools - Digital Image Speckle Correlation Tools. It is originated from the research work I participated in 1997 when I studied the parameters for digital image correlation. The algorithm was firstly written in Borland $\mathrm{C}++$, later I designed a better user interface using Borland $\mathrm{C}++$ Builder. It was distributed in several research institutes. It was re-designed recently for fast searching algorithm and easy omni-directional profile display, dual-direction displacement integration. The ability of process color and grayscale image, ASCII data, pseudo-color map, local data inspector, omni-directional profile display, dual-direction displacement integration, scale calibration and information display make this software handy and useful. By using multi-thread technique, multiple jobs could be submitted. Script file is also supported for large number of jobs. It is very useful for long time unattended calculation.

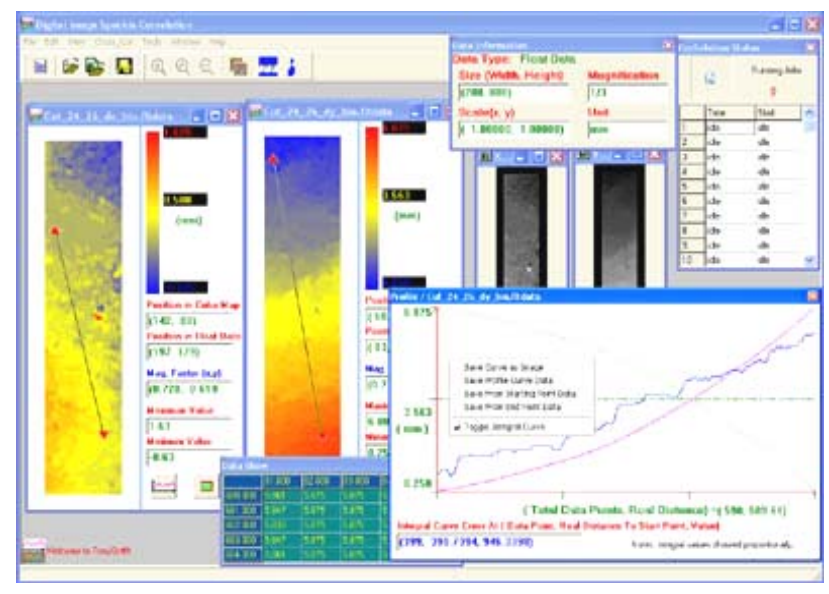

PM Tools - Photomechanics Tools. It is originated from a project for rock similar material research in 1999. It is also part of my master thesis. It has friendly user interface designed using Borland $\mathrm{C}++$ Builder. It is suitable for image analyses for most of the images obtained from Photomechanics. The processing ability includes Geometric moiré and Interferometry moiré fringe pattern, speckle image and grid image. Phase-shifting method, Fast Fourier Transformation (FFT) method, automated grid encoding method, and digital image correlation method are all implemented in the software. Results could be processed in binary and ASCII text format, display in grayscale and pseudo-color mode. For each method, detailed control panel give more control to the user. Flexible output control make the data easy be used for other software. It also includes advanced image/data processing tools, such as data recovery, edge detection, and area and volume estimation. 

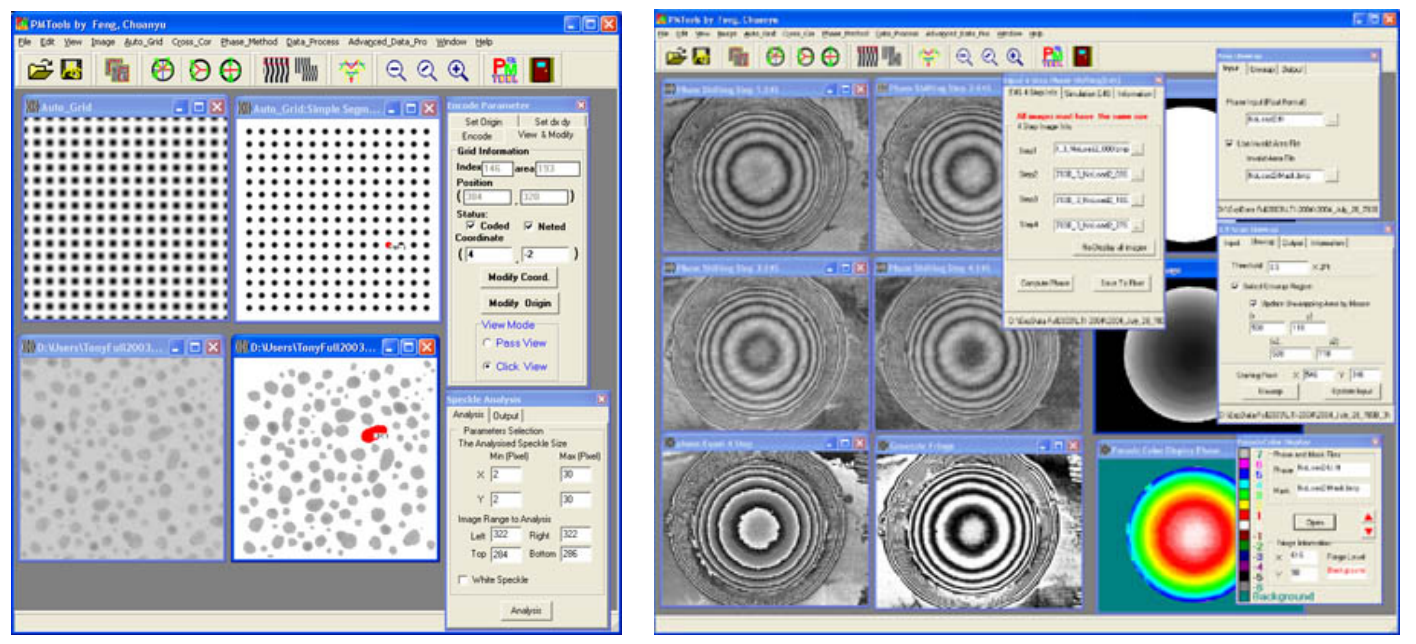

MPPM - Hardware related measurement and image processing tools (PI system, Matrox system and MIL-Lite). It was initiated in 1999 and become available in 2000 for a company in Shenyang, China. It was designed to measure the 3D profile of aircraft engineer leaf when I was a graduate student in China. It is based on Matrox Frame grabber (with dynamic configuration) and MIL-Lite as the image acquisition platform. For phase-shifting, it use PI's motor mover.

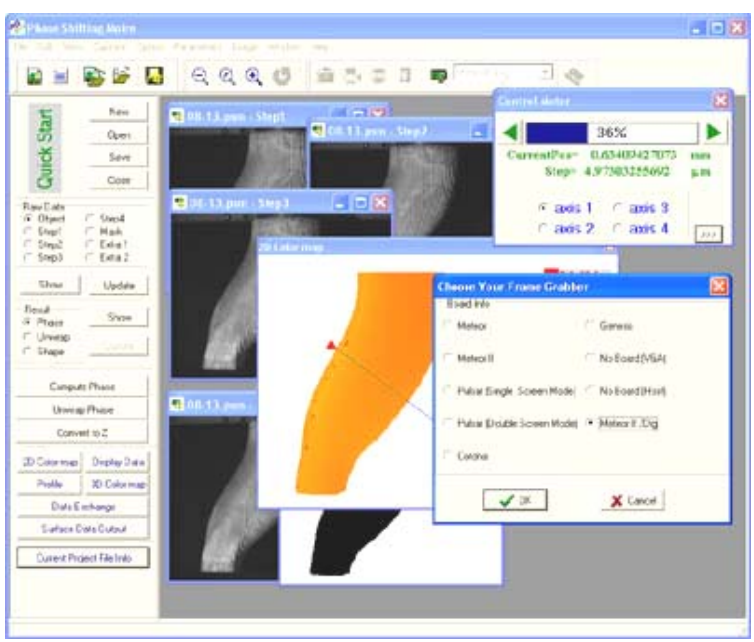

IMAQTools - Image acquisition and phase-shifting control for hardware from National Instruments and Meadowlark Optics. It was developed using Borland $\mathrm{C}++$ Builder and based on National Instruments IMAQ ${ }^{\mathrm{TM}}$ DLL library. The access to other hardware was through a shareware development kit NTPort ${ }^{\mathrm{TM}}$. Within one control panel, it can control Liquid Crystal Variable Retarder (LCVR) and image acquisition simultaneously. Using DLL (Dynamic Link Library) and multi-thread technique, IMAQTools can monitor CCD camera in real-time, apply desired voltage to the liquid crystal as necessary, automatically acquire images and save the images to pre-named files. It can also automatically display the phase map results which are very helpful when the vibration is of great concern.

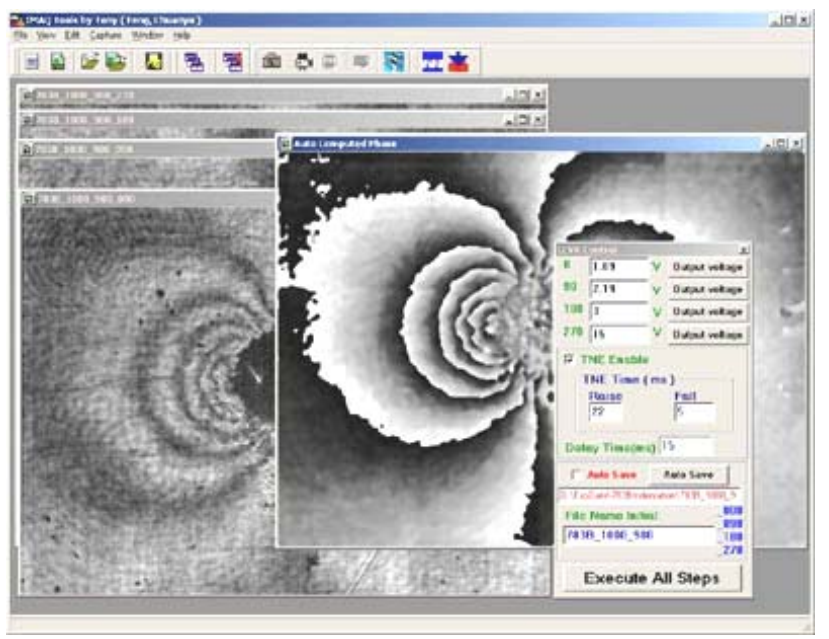

